Toward Functional Revascularization: Regulation of Vascular Network Remodeling Through Altered Hemodynamics

A Dissertation
Presented to
the faculty of the School of Engineering and Applied Science
University of Virginia
in partial fulfillment
of the requirements for the degree
Doctor of Philosophy
by

Joshua Keith Meisner

August

2013 
APPROVAL SHEET

The dissertation

is submitted in partial fulfillment of the requirements

for the degree of

Doctor of Philosophy

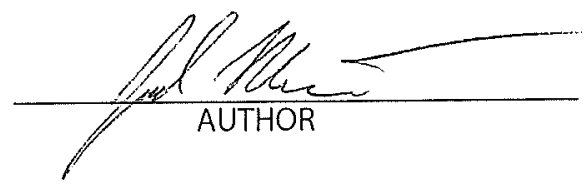

The dissertation has been read and approved by the examining committee:

\begin{tabular}{c}
$\frac{\text { Richard J. Price }}{\text { Advisor }}$ \\
Brett R. Blackman \\
\hline Brian H. Annex \\
\hline Michael B. Lawrence \\
\hline Gary K. Owens
\end{tabular}

Accepted for the School of Engineering and Applied Science:

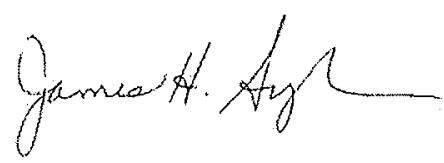

Dean, School of Engineering and Applied Science

August

2013 


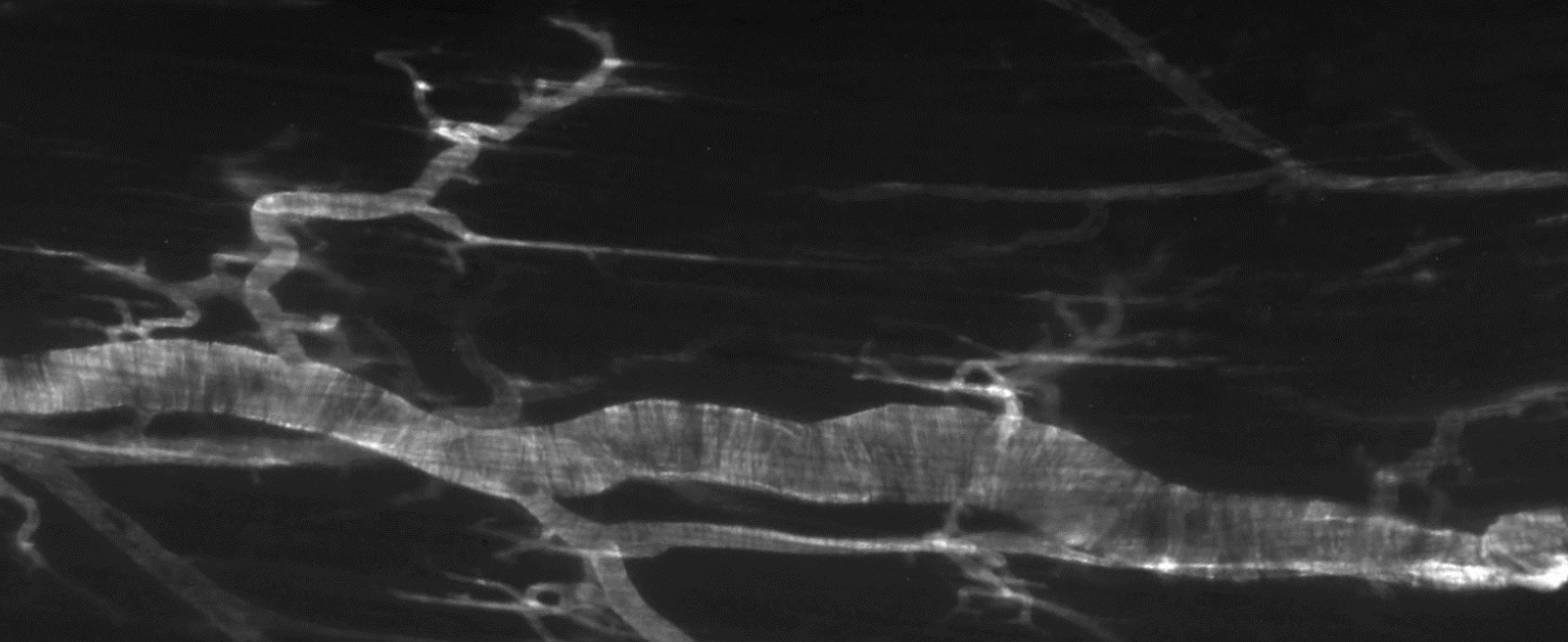

Toward Functional Revascularization Regulation of Vascular Network Remodeling Through Altered Hemodynamics

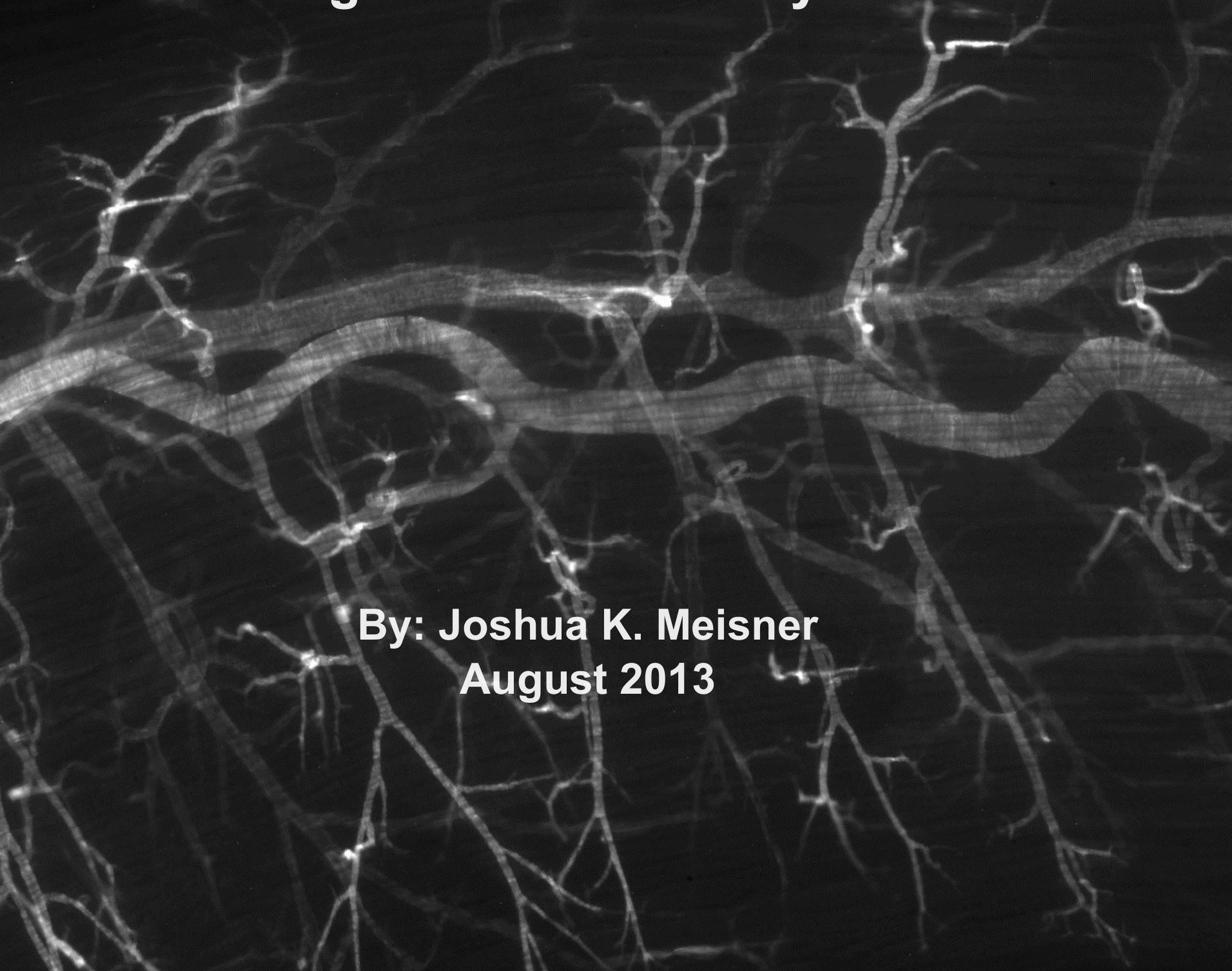




\title{
Toward Functional Revascularization: Regulation of Vascular Network Remodeling Through Altered Hemodynamics
}

\author{
By \\ Joshua Keith Meisner \\ August 2013 \\ In Partial Fulfillment \\ Of the Requirements for the Degree \\ Doctor of Philosophy in Biomedical Engineering \\ University of Virginia
}

Thesis Advisor:

Examination Committee:
Richard Price, Ph.D.

Brian Annex, M.D.

Brett Blackman, Ph.D., chair

Michael Lawrence, Ph.D.

Gary Owens, Ph.D 


\section{Specific Aims and Executive Summary:}

In ischemic tissues downstream of an arterial occlusion, capillaries grow from existing vessels via angiogenesis. In contrast, collateral arteries around the occlusion undergo structural lumenal expansion (arteriogenesis). While it is known that mechanical stimuli from altered hemodynamics and bone marrow-derived cells (BMCs) play different individual roles in angiogenesis (1) and arteriogenesis (2), how these elements interact to guide the remodeling of the peripheral vasculature to reperfuse the downstream ischemic tissue is largely unknown and intensely debated (see reviews (3-5)). Improper understanding of the balance of angiogenesis versus arteriogenesis and the distribution of stimuli during remodeling has been cited as the primary reason for the failure of numerous clinical trials targeting therapeutic revascularization efforts toward treating peripheral arterial disease $(P A D)(4,6,7)$. These vascular remodeling elements are further interwoven with the endogenous capacity to tolerate ischemia and regenerate tissue for a full functional compensation to PAD. Given the prevalence (>20\% of those $>65$ years of age (8)) and economic impact ( $\$ 4.4$ billion estimated treatment costs $(9)$ ) of PAD, there is a critical need to identify better targets for therapeutic intervention both in terms of the types of remodeling to promote and the molecular targets for accelerating the growth needed for a functional recovery.

To meet this problem, the following research plan was used to address several key issues in the field that are needed to develop effective revascularization therapies. First, we proposed using two separate mouse models to determine, for the first time, how arteriogenesis, angiogenesis, and muscle regeneration quantitatively contribute to reperfusion after arterial occlusion in order to identify the relative importance of targeting each type of remodeling for revascularization therapies (Aim 1). Second, we developed and validated the use of quantitative laser speckle flowmetry (LSF) as a tool for determining how 
the distribution of hemodynamic changes guides arterial remodeling (Aim 2). Finally, preliminary data from our laboratory suggested that regional variation in pre-existing hemodynamic directionality determines the rate and capacity for arteriogenesis across a network. Using the novel tools developed to map hemodynamic change across a network at the arteriole level, we produced the first direct measurements of the changes in shear stress that drive the arteriogenic process. From these novel data, we demonstrated how differential mechanotransduction due to pre-existing endothelial polarity functions as an independent enhancer of arteriogenesis that can be used to identify critical pathways for enhancing collateral development (Aim 3).

\section{Aim 1: Determine the contribution of angiogenesis and skeletal muscle regeneration} to reperfusion after arterial occlusion in the ischemic mouse hindlimb model. Hypothesis: Mouse models of femoral artery ligation (FAL) that exhibit specific impairment in distal angiogenesis or skeletal muscle regeneration, but normal collateral arteriogenesis, will still demonstrate a significant decrease in reperfusion. First, we used a bone marrow specific knockout of MMP9 mouse model that we hypothesized had normal angiogenesis and arteriogenesis, but reduced skeletal muscle regeneration after FAL to test if impairment of skeletal muscle regeneration alone is sufficient to significantly impair the reperfusion response to arterial occlusion (Chapter 2). Second, we use a transgenic model of myoglobin overexpression that we hypothesized had impaired angiogenesis but normal arteriogenesis after FAL to test if impairment of angiogenesis alone is sufficient to significantly impair the reperfusion response to arterial occlusion (Chapter 3).

\section{Aim 2: Develop laser speckle flowmetry (LSF) technology for determining the distribution of changes in hemodynamics throughout arteriolar networks. Technology Development Goal: Validate the combination of intravital microscopy with LSF for use in quantifying hemodynamic changes in superficial (epi-illumination) and deep (trans-}


illumination) arteriolar networks. We first validated the quantitative in vivo potential of LSF for use with intravital microscopy using particle imaging velocimetry. Second, we applied the technology to demonstrate LSF can be used to map network hemodynamics and collateral arteriole recruitment after arteriole occlusion in the mouse dorsal skinfold window chamber (Chapter 4). Third, we determined the potential of trans-illumination LSF for imaging deeper vascular networks for application to the primary collateral network of the mouse upper hindlimb after FAL. From this data, we produced the first ever map of hemodynamic changes in this widely used model of arteriogenesis and angiogenesis. These data served as the basis for the in vitro translation of Aim 3 (Chapter 5).

\footnotetext{
Aim 3: Determine if differential directional stimulus leads to accelerated arteriogenesis after arterial occlusion based off of pre-existing hemodynamic patterns. Hypothesis: Upon arterial occlusion, endothelial cells (ECs) in select regions of a collateral artery pathway experience a reversal in shear stress with respect to their pre-align direction, and this directional reversal enhances the arteriogenic response to increased shear stress. First, we determined that regionally accelerated growth in the primary collateral network in the upper hindlimb correlated with a change in shear stress direction with respect to pre-existing EC polarization. Second, we applied the regional changes in shear stress in terms of magnitude and direction measured in vivo to cultured ECs. We demonstrated that directional change opposite of pre-aligned EC polarization elicited a broad enhancement of the mechanotransduction response to increase in shear stress, which is argued to be the central driving stimulus in arteriogenesis (Chapter 5).
} 


\section{TABLE OF CONTENTS:}

Acknowledgements......................................................................

Chapter 1: Spatial and Temporal Coordination of Bone MarrowDerived Cell Activity During Arteriogenesis: Regulation of the Endogenous Response and Therapeutic Implications................ 1

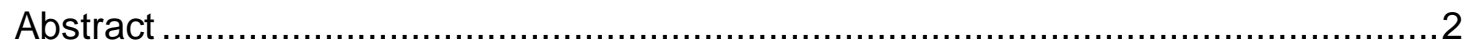

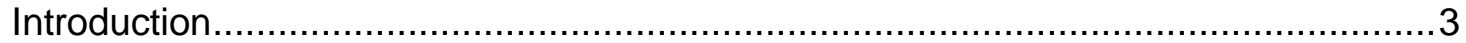

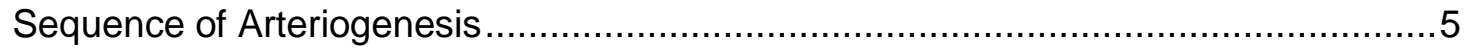

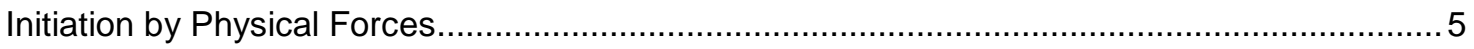

Propagation of Growth Process by Inflammation................................................................. 7

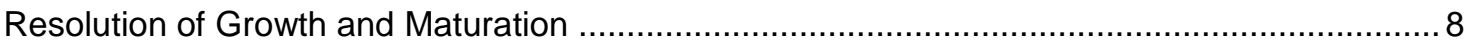

Leukocyte Types and Temporal Recruitment In Arteriogenesis ...........................9

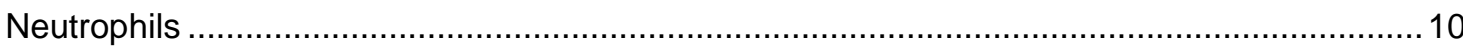

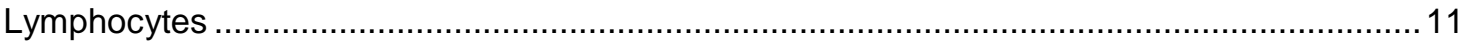

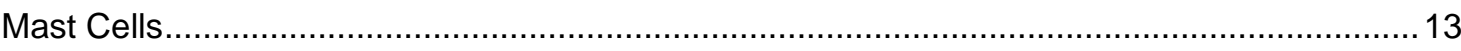

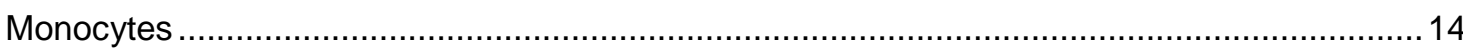

Bone Marrow Derived Progenitor Cells ............................................................................. 17

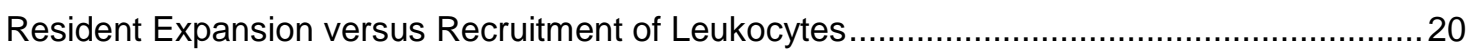

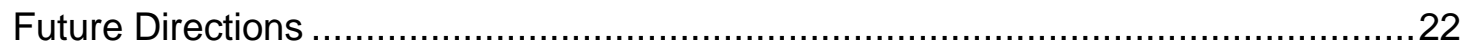

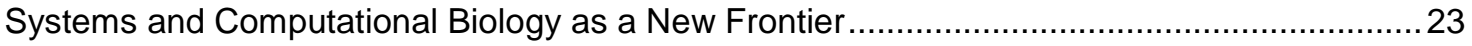

Identifying the Common Impairments to Collateral Growth in Patients ....................................24

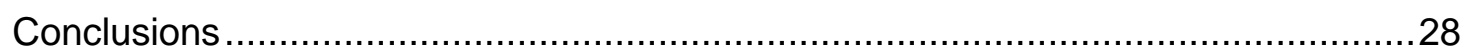

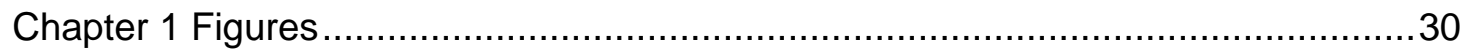

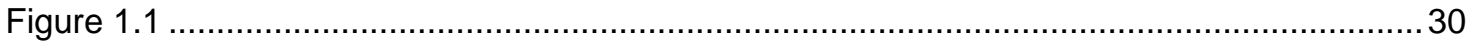

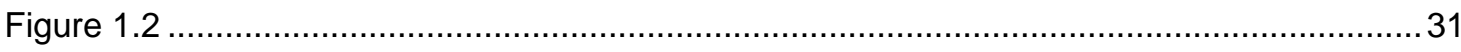

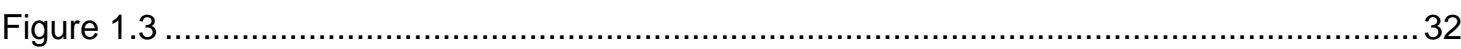

Chapter 2: Bone Marrow Cell-Derived Matrix Metalloproteinase 9 Deletion Inhibits Reperfusion in the Ischemic Mouse Hindlimb Through Impaired Skeletal Muscle Regeneration, but Does Not Affect Arteriogenesis and Arteriogenesis.

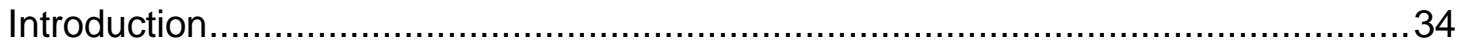

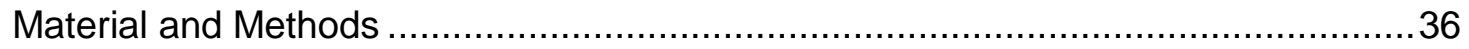

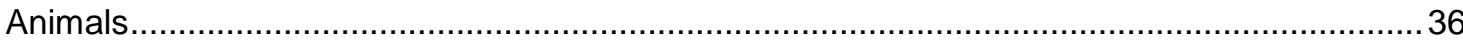

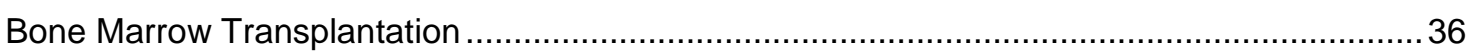




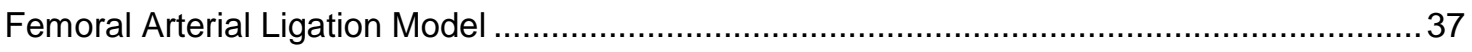

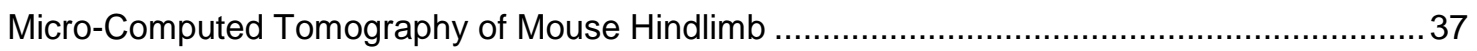

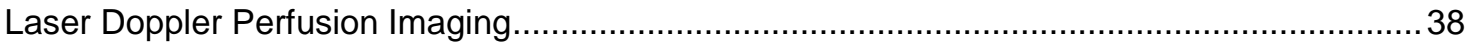

Tissue Harvesting for Cross-sectional Analysis and Collateral Network Structure ....................38

Whole Mount Immunofluorescence and Collateral Network Image Analysis ...........................38

Cross Sectional Analysis of Collateral Structure and Collagen ............................................ 39

Immunohistochemistry Analysis of Immune Cell Recruitment ................................................ 40

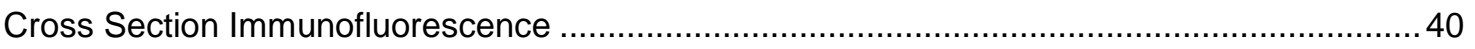

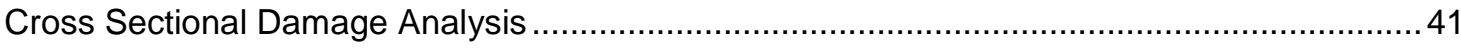

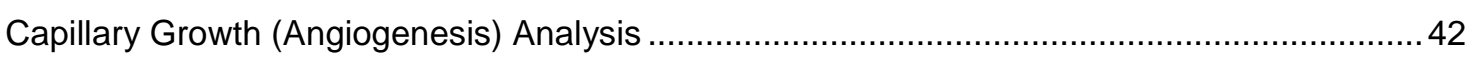

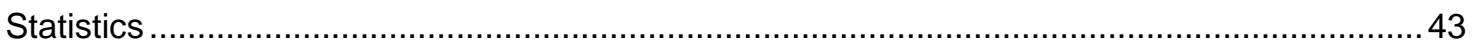

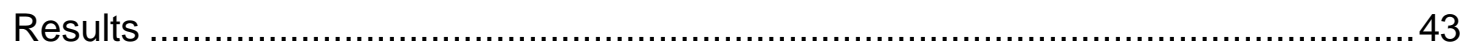

Loss of BMC-Derived MMP9 Impairs Perfusion Recovery After FAL ...................................... 43

Loss of BMC-Derived MMP9 Does Not Alter Arteriogenesis in Response to FAL .................... 43

Loss of BMC-Derived MMP9 Does Not Alter Peri-Collateral Collagen Remodeling in Response

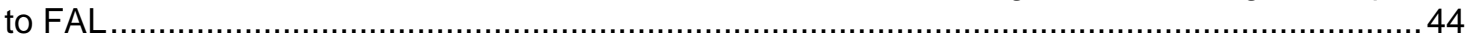

Loss of BMC-Derived MMP9 Does Not Alter Leukocyte or Mast Cell Recruitment.................... 45

Loss of BMC-Derived MMP9 Does Not Alter Angiogenesis in Response to FAL .....................45

Loss of BMC-Derived MMP9 Impairs Ischemic Skeletal Muscle Recovery after FAL ...............48

Impaired Perfusion Recovery is Associated With Impaired Ischemic Skeletal Muscle Recovery

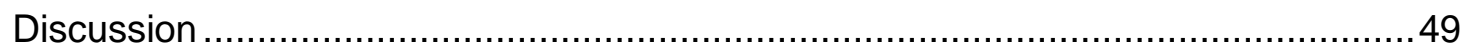

Summary

Comparison to previous studies and the need for detailed assessment of angiogenesis and

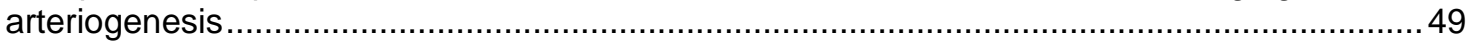

Potential Mechanisms of MMP9 in impaired muscle repair ................................................ 51

Importance of skeletal muscle regeneration in vascular function .............................................52

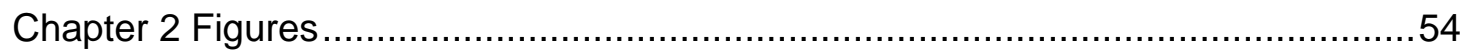

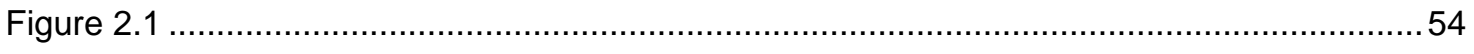

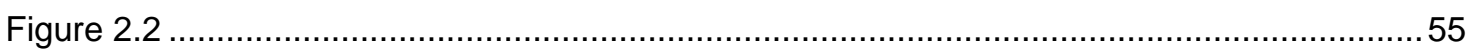

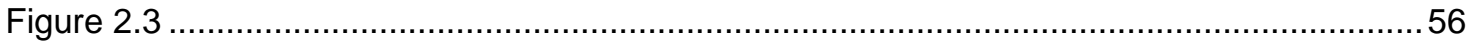

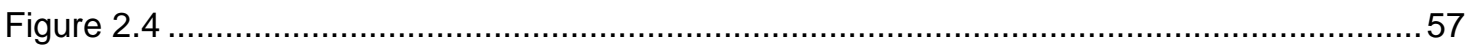

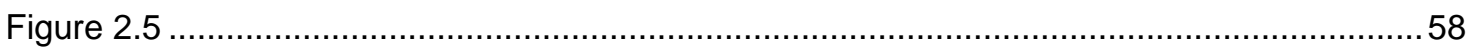

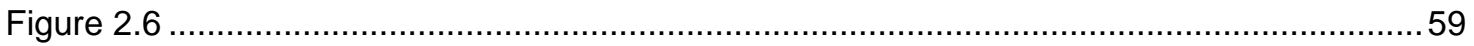

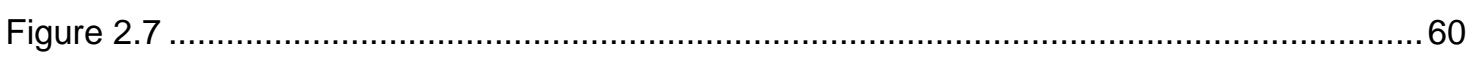

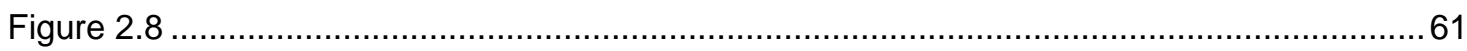

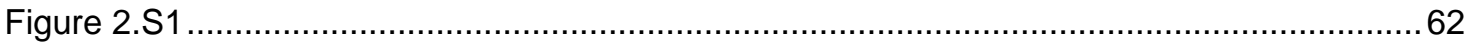

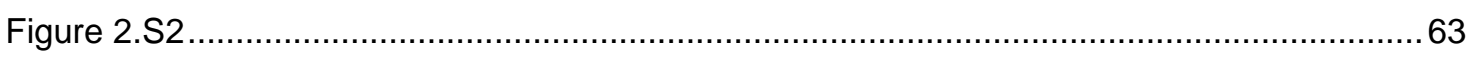




\section{Chapter 3: Myoglobin Overexpression Inhibits Reperfusion in the Ischemic Mouse Hindlimb Through Impaired Angiogenesis but Not Arteriogenesis.

Introduction .65

Material and Methods 67

Animals

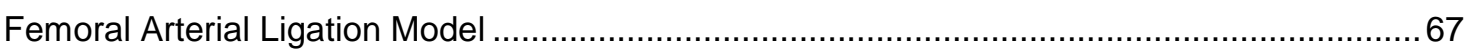

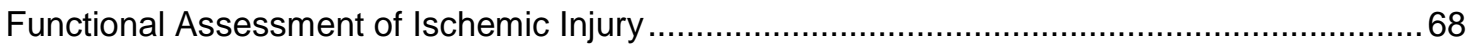

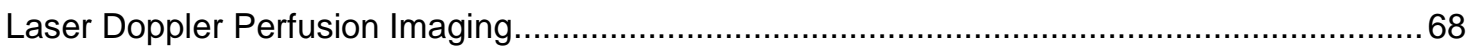

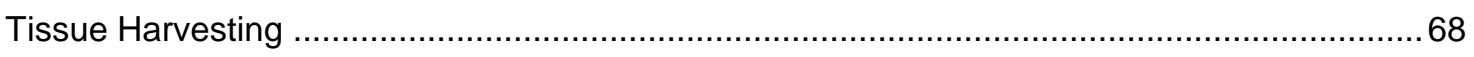

Whole Mount Immunofluorescence and Collateral Network Image Analysis ............................69

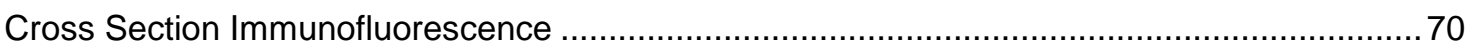

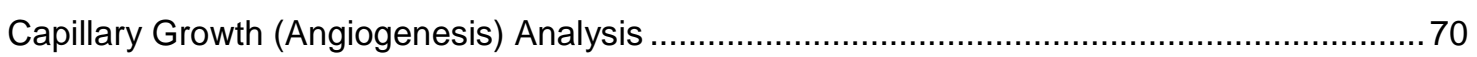

Cross Sectional Damage Analysis of Ischemic Tissue ...................................................... 71

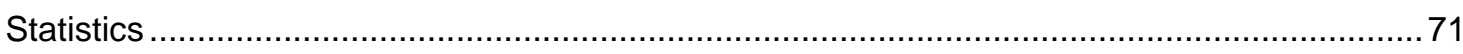

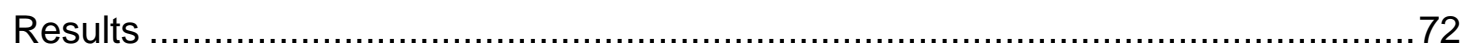

Myoglobin Over-expression Impairs Perfusion and Functional Recovery after FAL …..............72

Myoglobin Over-expression Does Not Alter Arteriogenesis in Response to FAL ......................72

Myoglobin Over-expression Delays Skeletal Muscle Recovery, but Does not Alter Capillary

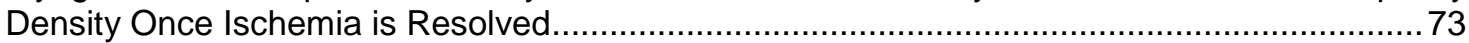

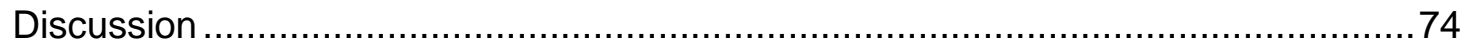

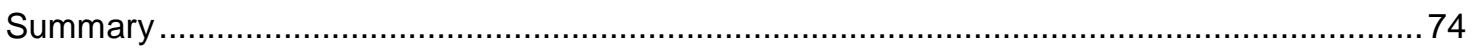

Myoglobin Overexpression Preferentially Impacts Glycolytic Muscle Fibers ............................ 75

Relative Contribution of Arteriogenesis and Angiogenesis ............................................... 75

Clinical Impetus for a Combined Role of Arteriogenesis and Angiogenesis in PAD .................77

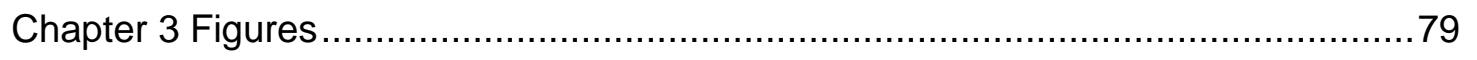

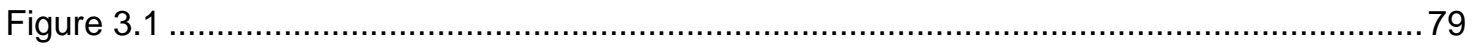

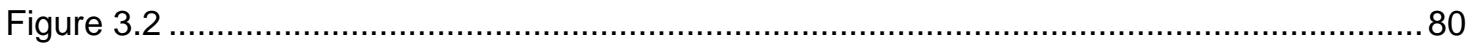

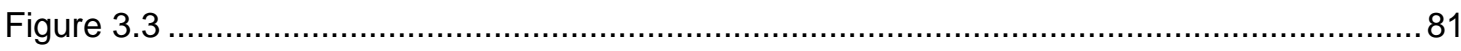

\section{Chapter 4: Laser Speckle Flowmetry Method for Measuring Spatial and Temporal Hemodynamic Alterations Throughout Large Microvascular Networks. ............................................................ 82}

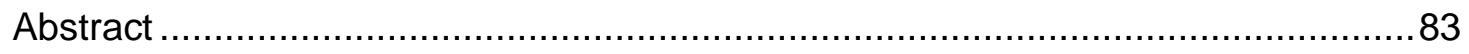

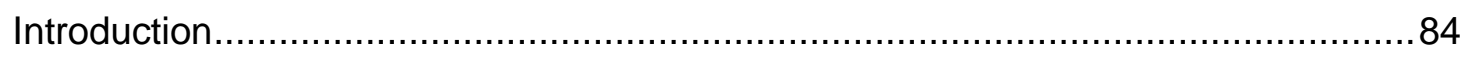

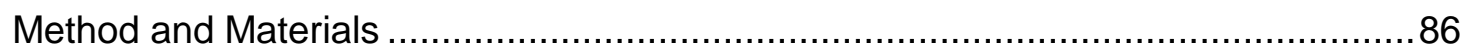

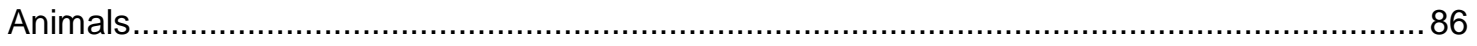

Dorsal Skinfold Window Chamber Implantation for Intravital Imaging .................................. 86 


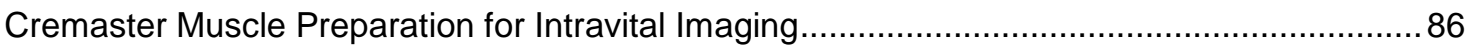

Laser Speckle Flowmetry for Imaging of Microvascular Network Flow .................................... 87

In Vitro Speckle Tissue Phantom Imaging Protocol ............................................................. 89

In Vivo Calibration Imaging Protocol Using Laser Speckle and Microspheres ........................... 89

In Vivo Measurement of Altered Network Flow Using Laser Speckle......................................90

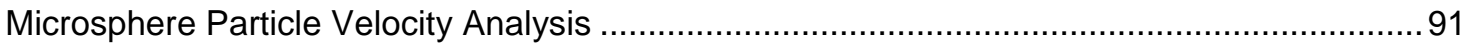

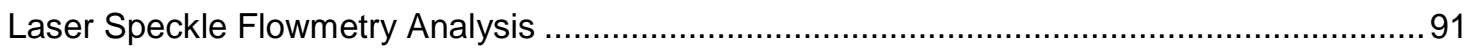

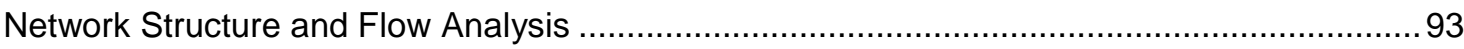

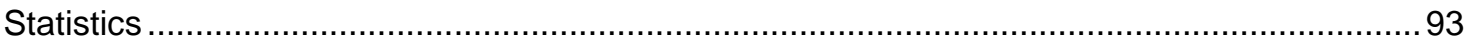

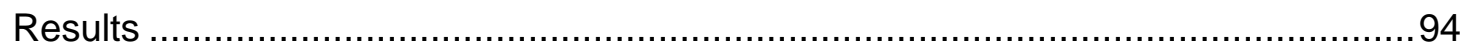

In Vitro Testing of Laser Speckle Flowmetry Linear Response ..............................................94

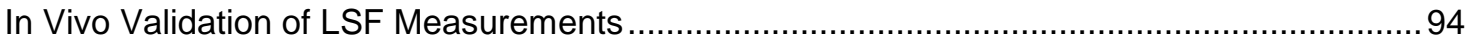

Application of LSF and Brightfield Intravital Imaging to Large Microvascular Networks............95

Alteration in Network and Collateral Pathway Hemodynamics in BALB/C and C57BL/6 Mice ..96

Discussion

Laser Speckle Flowmetry as a Quantitative Tool for Absolute Measurements ........................97

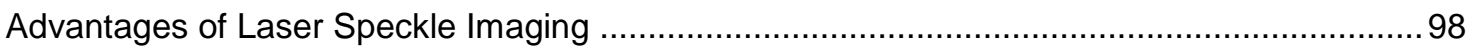

Use of LSF for Interrogating Network Responsiveness to Microvessel Occlusion in C57BL/6

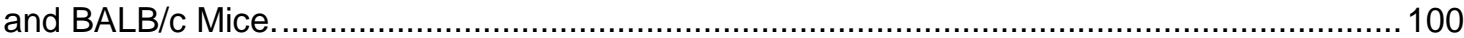

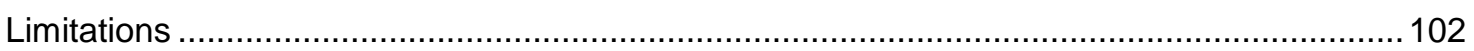

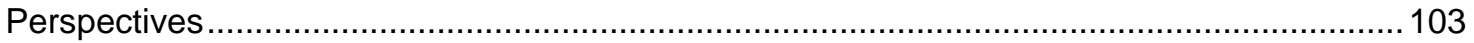

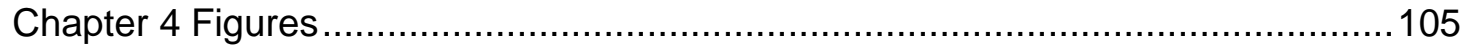

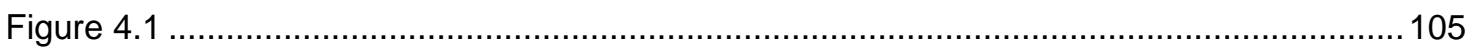

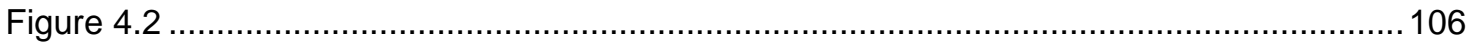

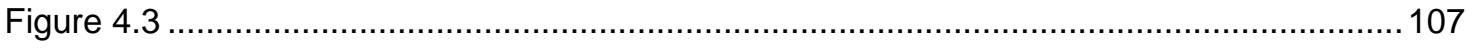

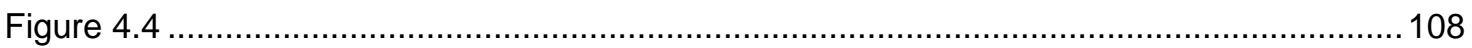

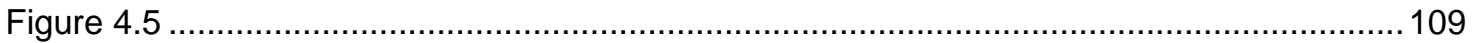

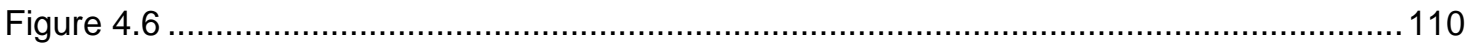

\section{Chapter 5: Trans-illuminated Laser Speckle Imaging of Deep Collaterals in Ischemic Mouse Hindlimb Reveals that Flow Direction Reversal is an Independent Arteriogenic Stimulus .. 111}

Introduction. 112

Materials and Methods 114

Supplemental Methods

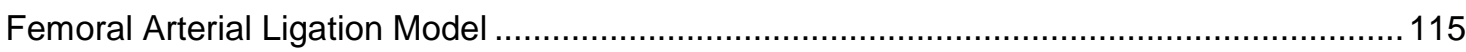

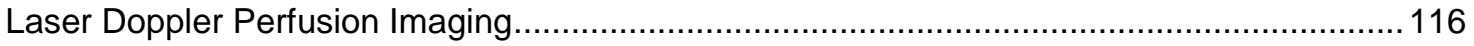

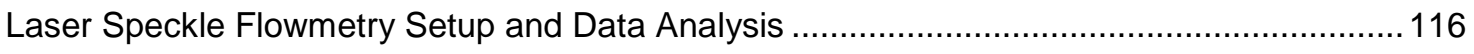

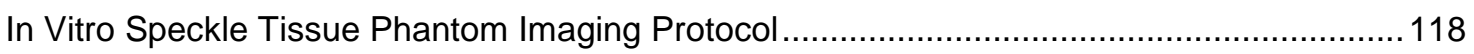


In vivo analysis in transillumination hindlimb

Tissue Harvesting for Cross Sectional Analysis and Collateral Structure by Vascular Casting

Tissue Harvesting for Collateral Structure by Immunofluorescence .....................................121

Tissue Harvesting for In Vivo Collateral Artery Endothelial Planar Cell Polarization................ 122

Cross Sectional Analysis for Regional Capillary Density and Muscle Fiber Atrophy................123

Regional Analysis of Collateral Artery Structure and Hemodynamics ................................... 124

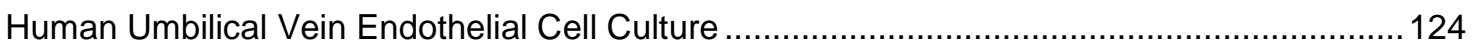

In vitro Exposure of Endothelial Cells to Simulated Ligation Shear Stress ............................ 125

HUVEC RNA isolation, qRT-PCR, and Microarray Gene Expression Profiling ....................... 125

Immunofluorescence Labeling for HUVEC Planar Cell Polarity ........................................... 127

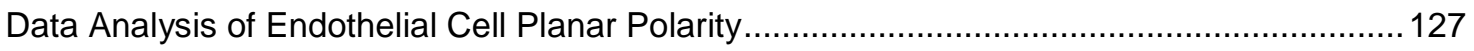

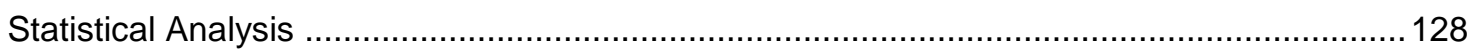

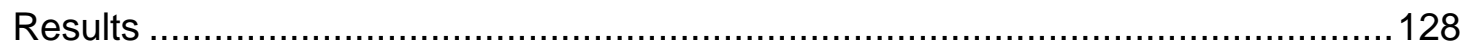

Transillumination Laser Speckle Flow Microscopy Decreases the Signal Attenuation from

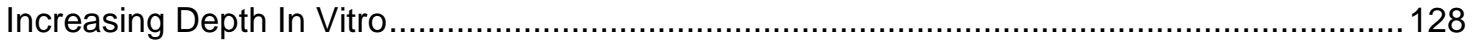

Transillumination LSF Microscopy Demonstrates Regional Variation of Hemodynamic Changes Along Collateral Arteries After Femoral Artery Ligation. ................................................. 129

Gracilis Collateral Arteries Exhibit Increased Arteriogenesis in Flow Reversal Regions......... 130

No Evidence of Hypoxia in Developing Collateral Regions .................................................. 132

Endothelial Cell Planar Polarization Demonstrates Orientation and Reorientation to Predicted

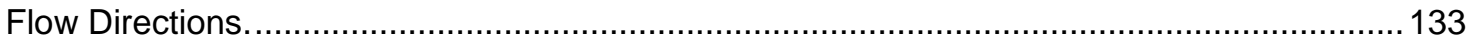

HUVECs Exhibit Planar Polarization and Directional Responsiveness to Simulated Femoral

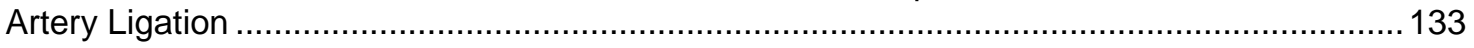

Flow Direction Reversal Broadly Enhances the Arteriogenic Gene Expression Pattern Seen

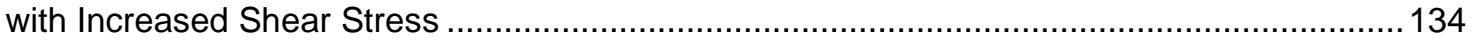

Flow Reversal With Increased Shear Stress Magnitude More Fully Activates Key Arteriogenic Pathways When Compared to Increased Shear Stress Magnitude Alone .............................135

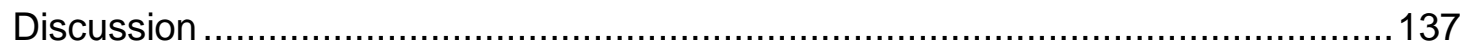

Application of Transillumination LSF for Generating Novel Measurements of In Vivo

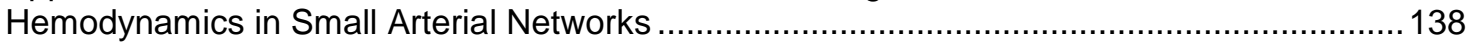

Spatial Measurements of Altered Hemodynamics and Growth Identify Flow Reversal as a

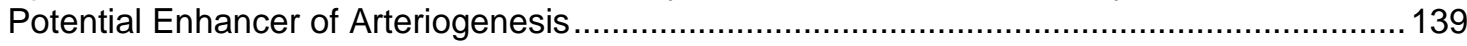

Endothelial Cell Cytoskeletal Repolarization from a Pre-Conditioned Baseline As A Potential Mechanism of Enhanced Arteriogenic Signaling …............................................................. 140

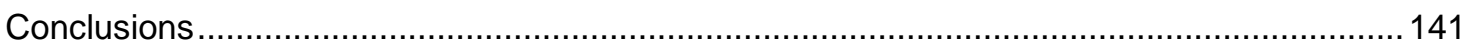

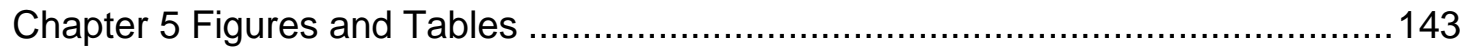

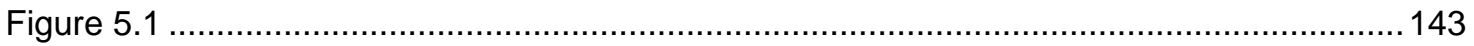

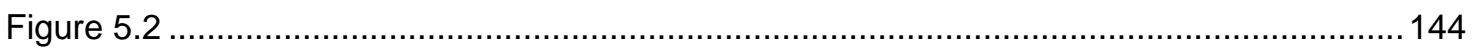

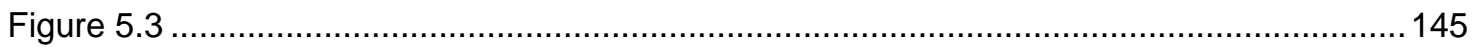

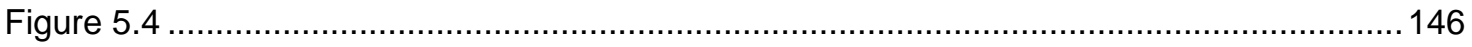

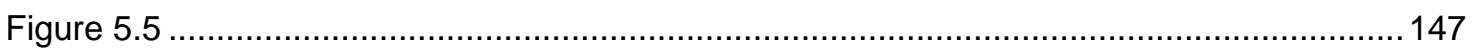




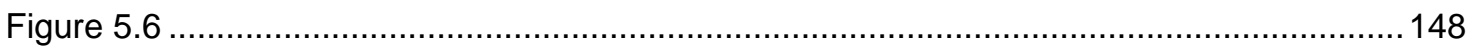

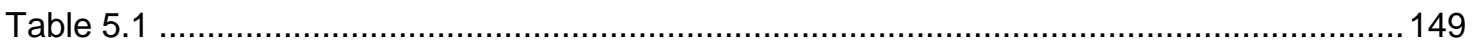

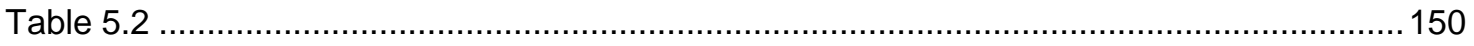

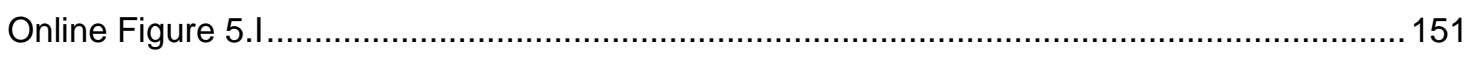

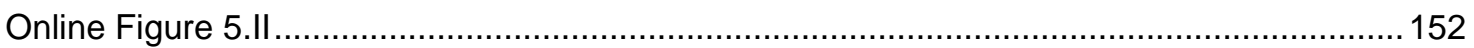

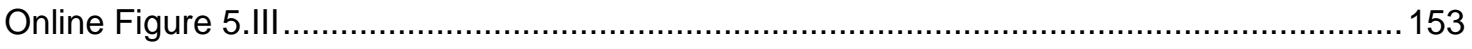

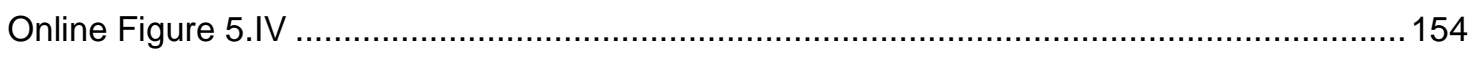

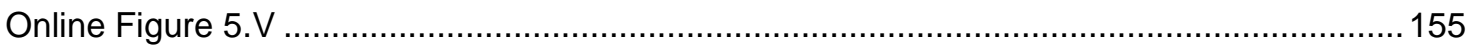

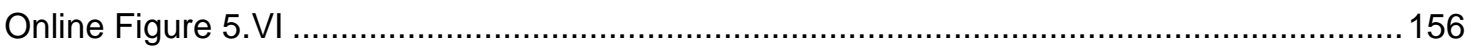

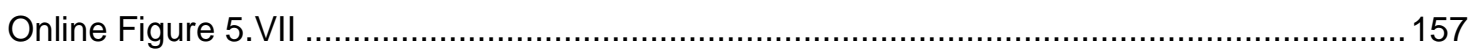

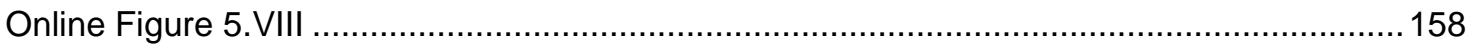

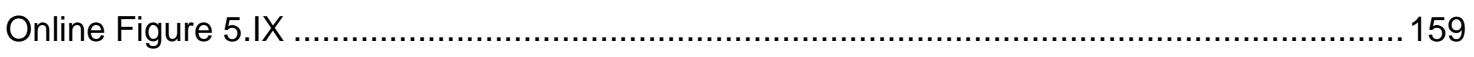

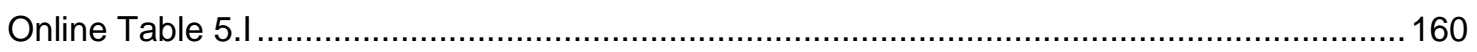

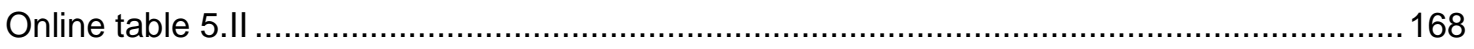

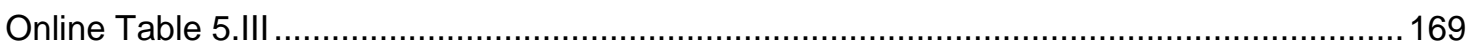

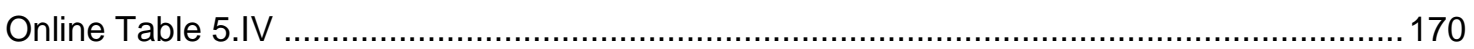

\section{Chapter 6: Thoughts on the Direction of Future Research Projects} .................................................................................................. 171

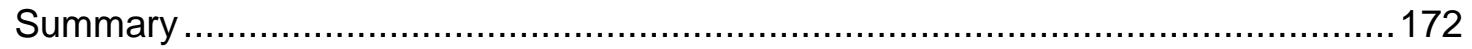

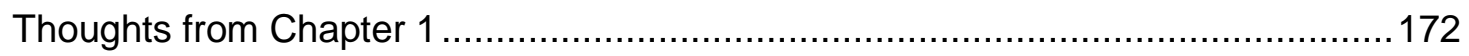

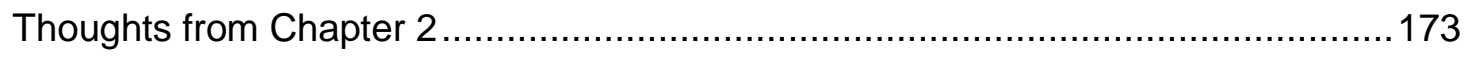

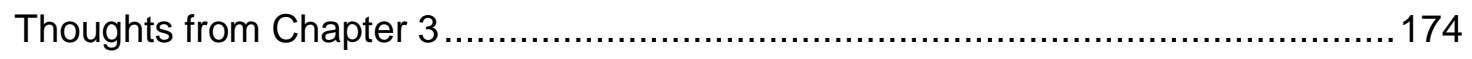

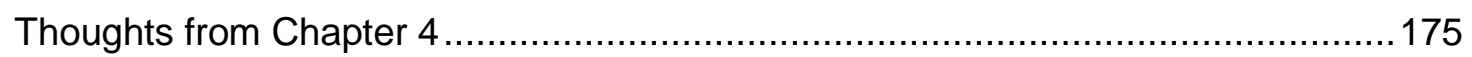

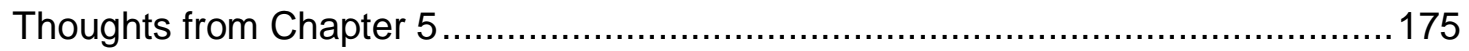

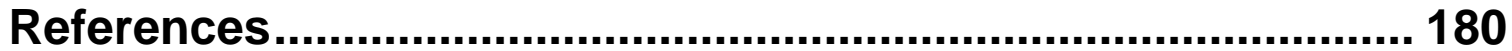




\section{Acknowledgements}

Throughout my pursuit of this work, I have received an immeasurable amount of help and support. I am truly grateful for the mentorship of my advisor, Dr. Richard Price. His support and guidance along the way provided a unique opportunity to explore and discover my own path that I have seen replicated nowhere else. He is the model for the advisor I hope to be. Thank you for the patience and tolerance to let me reach out into the unknown.

To my committee members Drs. Gary Owens, Brian Annex, Brett Blackman, and Michael Lawrence, thank you for your service. The advice in both science and career have helped shape my perspectives and given me a foundation from which to pursue the next step.

To all of the past and present members from the Price, Skalak, and Peirce-Cottler laboratories not to mention the other dozen laboratories I have had the pleasure to work with, thank you. To Josh Heuslein and Ji Song, in particular, thank you. This work itself is a tribute to the team science and collaboration that is at the core of the University of Virginia. May this place continue to nurture the next generation of scientists.

For all of the undergraduate students that chose to place their faith in me as an advisor, thank you. Their questions and thoughts have encouraged and challenged me to grow as a teacher and advisor. Their hard work has both inspired me and been a cornerstone in advancing this work.

I am deeply grateful to my family who have supported and encouraged me throughout my life. Finally, no words can truly express my gratitude to my wife, Elizabeth, for celebrating in my successes and encouraging me during my struggles. I have looked to her as an inspiration in innumerable ways. She has been and always will be my equal and partner on this path together. 
Chapter 1: Spatial and Temporal Coordination of Bone MarrowDerived Cell Activity During Arteriogenesis: Regulation of the Endogenous Response and Therapeutic Implications

Joshua K. Meisner, Richard J. Price. Microcirculation 17: 583-599, 2010 


\section{Abstract}

Arterial occlusive disease (AOD) is the leading cause of morbidity and mortality through the developed world, which creates a significant need for effective therapies to halt disease progression. Despite success of animal and small-scale human therapeutic arteriogenesis studies, this promising concept for treating AOD has yielded largely disappointing results in large-scale clinical trials. One reason for this lack of successful translation is that endogenous arteriogenesis is highly dependent on a poorly understood sequence of events and interactions between bone marrow derived cells (BMCs) and vascular cells, which makes designing effective therapies difficult. We contend that the process follows a complex, ordered sequence of events with multiple, specific BMC populations recruited at specific times and locations. Here we present the evidence suggesting roles for multiple BMC populations_from neutrophils and mast cells to progenitor cells-and propose how and where these cell populations fit within the sequence of events during arteriogenesis. Disruptions in these various BMC populations can impair the arteriogenesis process in patterns that characterize specific patient populations. We propose that an improved understanding of how arteriogenesis functions as a system can reveal individual BMC populations and functions that can be targeted for overcoming particular impairments in collateral vessel development.

Keywords: arteriogenesis, leukocytes, bone marrow-derived cells, collateral vessels, recruitment sequence 


\section{Introduction}

Arterial occlusive disease is already the leading cause of morbidity and mortality in the developed world with as much as $20 \%$ of the aged population having asymptomatic peripheral arterial disease (8). Moreover, the spread of Western lifestyle will only increase the disease prevalence in the coming decades. Since the $18^{\text {th }}$ century, it has been known that there is a preexisting network of bridging collateral arteries capable of compensating for major arterial occlusion (3). With growth, these collateral vessels can provide alternate routes for blood flow around occluded arteries, preventing ischemic injury in downstream tissues. This observation and numerous subsequent studies over the last 40 years have raised the promise of therapeutically driving the process of collateral vessel growth for the treatment of a variety of arterial occlusive diseases. However, many of the large-scale therapeutic arteriogenesis clinical trials have yielded disappointing results in humans. This is despite the promising initial animal studies and pilot human studies (see reviews (10-13)). One of the key lessons from these failures is that, to develop a more effective therapy, we need to improve our basic understanding of the coordinated process of arteriogenesis $(6,11,13)$.

The arteriogenic process is a highly orchestrated process involving the recruitment and proliferation of numerous cells types and the reorganization of the extracellular matrix, all of which are coordinated through a temporal pattern of cytokine, chemokine, growth factor, and protease expression. It is not surprising, therefore, that the application of single growth factors or therapeutic agents, irrespective of the specific mechanism and sequence they contribute to the arteriogenesis pathway, have not been therapeutically successful. One promising avenue of targeted treatment that may help recapitulate the coordinated arteriogenic process is the use of bone marrow derived cells to aid the growth of collateral vessels. 
Since 1976 (14), it has been known that developing collateral vessels are prominently invested with bone marrow derived cells in a perivascular position. Subsequent study has shown these cells to be essential in coordinating the complex sequence of events necessary in driving collateral vessel growth (10). However, initial clinical trials targeting BMC activation and mobilization (e.g. GM-CSF $(12,15)$ and G-CSF (16-18)) have fared little better than single growth factor trials. Part of the reason for the lack of success may arise from not having considered patients' impairments in the arteriogenic pathway (11) and/or how the cell types and methods used fit into the arteriogenic process. Given the interconnected nature of the arteriogenic pathway, impairments in any number of steps in the process can result in the same downstream results of insufficient collateral growth. Further, understanding the time-course of interactions of BMCs and vascular cells can highlight the processes occurring during the different phases of arteriogenesis and provide insight into how a proposed therapy might function within the system of pathways that drive collateral vessel growth. Moreover, understanding the temporal pattern of various populations of BMC accumulation may provide a means of identifying specific cell populations that can be used to target specific phases of arteriogenesis. Therefore, in this article, we will discuss the studies that have begun to elucidate the roles of BMCs in coordinating collateral vessel growth, the questions left unanswered, and how understanding the timing of how cells that coordinate the arteriogenic process may benefit the development of future therapeutic arteriogenesis efforts. First, we will outline the process and phases of arteriogenesis. Second, we will discuss the BMC populations involved during the arteriogenic process and the spatial and temporal interactions of BMCs with each other and vascular cells. Lastly, we will discuss how impairments could arise in any number of these pathways and the potential implications of using a view that encompasses the system of responses in arteriogenesis when designing future therapeutic strategies. 


\section{Sequence of Arteriogenesis}

By first examining the overall process of arteriogenesis, we can begin to see the spatial and temporal complexity and the overlap of cellular and molecular systems that abound throughout the process. Overall, the sequence of arteriogenesis can be divided into three phases: initiation, growth, and maturation (Figure 1.1). Each phase involves the interplay of physical forces, leukocyte recruitment, extracellular matrix remodeling, and numerous growth and signaling cascades.

\section{Initiation by Physical Forces}

Numerous studies have now identified changes in the physical forces experienced by endothelial cells to be a major initial stimulus in triggering collateral vessel growth (see review (19)). In response to the occlusion of a major artery, such as caused by an atherosclerotic plaque in a pathological state or an acute ligation in an experimental model, the vascular network downstream of the occlusion experiences a drop in pressure. This distal drop in pressure creates a steep pressure gradient that drives flow along any smaller, pre-existing bridging arteries that circumvent the occlusion. The resulting increase of flow along these collateral vessels is partially able to resupply the downstream tissue, but due to the increased length of the vascular path and reduced radius, the resistance to flow is much greater. This higher resistance network of pre-existing collateral vessels results in a sustained low perfusion pressure distal to the occlusion site and maintains the steep pressure gradient along the collateral pathways. This distal under-perfusion remains until the network has remodeled its structure to minimize resistance. The viscous force of the increased blood flow along these initial bridging arteries creates a shear stress at the wall that endothelial cells can sense, triggering a cascade of events initiating the arteriogenic process. The decreased perfusion pressure in the distal tissues, however, provides the additional vascular remodeling stimulus of 
ischemia, especially in the form of angiogenesis driven by HIF1a stabilization. Therefore, upon occlusion, the redistribution of resistance and pressure gradients throughout the network provides the stimulus for driving arteriogenesis in collateral vessels and angiogenesis in the downstream ischemic tissue.

However, the locations of these driving stimuli do not always spatially coincide, and these stimuli evolve over time as the network remodels. Several groups have shown that in the most common experimental model for studying collateral growth (i.e. femoral arterial ligation), the angiogenic stimulus of hypoxia is not present in the primary tissues where collateral growth is occurring (20-22). As the arteriogenic process progresses, multiple parallel collateral vessels undergo lumenal expansion, which decreases overall network resistance and restores the driving perfusion pressure to the distal tissues. The lumenal expansion returns collateral vessel shear stress to a stable value, which allows for vessel maturation. When shear stress does not return to "normal," collateral vessels will continue to remodel and expand as is seen in a shunt model of collateral growth (23-25). Conversely, as the collateral network remodels and network resistance decreases, distal perfusion pressure returns to normal. The stimulus for angiogenesis reverses, and capillary density returns to normal (26). It is important to note that the remodeling of collaterals has the greatest impact on overall network resistance and not the remodeling of capillaries in the distal ischemic tissue (27-29). Additionally, it is important to consider that changes in shear stress and ischemia are not mutually exclusive stimuli and are likely to both contribute to collateral growth (30). Ischemia may play a particularly prominent role in coronary collateral growth, where ischemia is in close proximity to developing collateral vessels (30). Moreover, even when ischemia and collateral growth are greatly separated, such as in the hindlimb ischemia model, distal ischemia appears able to affect upstream collateral growth through systemic mobilization of BMCs (31). However, the development of a mature collateral network is the primary means of reestablishing perfusion and changing shear stress 
stimuli throughout the vascular network, which dictates both the initiation and maturation of collateral vessel growth.

\section{Propagation of Growth Process by Inflammation}

The activation of the collateral vessel endothelium by a change in shear stress induces an inflammatory response that initiates and sustains the collateral growth process. In vivo and in vitro studies have shown that such a transient increase in shear stress induces the expression of a wide array of inflammatory cytokines, chemokines, and adhesion molecules $(21,32)$. Of particular importance is the shear stress-mediated upregulation and surface expression of adhesion molecules such as selectins, ICAM, and VCAM along the lumenal surface of collateral vessels (32). These adhesions proteins are known to be critical to inducing collateral growth primarily through the recruitment of leukocytes, such that removal or neutralization of these adhesion molecules abrogates arteriogenesis (33). Similarly, the upregulation of a variety of chemokines along collateral vessels (e.g. MCP-1), induces the recruitment of specific bone marrow-derived cell (BMC) populations that aid in the remodeling process (see review (34)). Additionally, the inflammatory signaling cascades induce the differentiation of collateral artery smooth muscle cells from a contractile phenotype to a synthetic phenotype, allowing for the subsequent growth phase and remodeling of the vessel wall extracellular matrix (32). Overall, the process results in the activation of intrinsic vascular cells that initiate the remodeling process leading to the accumulation of BMCs, whether in a model of coronary collateral growth (35) or hindlimb ischemia (32).

Once activated, these cells begin the growth process, which requires the coordination of numerous events to arrive at lumenal expansion of collateral vessels. Initially, the existing basement membrane components, such as desmin and laminin, and the internal elastic lamina are broken down by matrix proteases, particularly matrix metalloproteases 2 and 9 (36-38). 
These components are replaced by a new transitional extracellular matrix, including FN (37), which may help propogate the inflammatory response especially during changes in shear stress (39). This matrix remodeling also allows for the outward migration and proliferation of vascular cells. Concurrently, numerous growth signals are released from the breakdown of extracellular matrix $(40-42)$ and triggered by altered matrix components $(37,38)$ along with the release of paracrine signaling molecules from bone marrow derived cells $(43,44)$. These various signaling cascades trigger the differentiation and proliferation of vascular cells. At each stage, inflammatory pathways are the driving forces for the signaling process with recruited BMCs appearing to play an enabling role at each step.

\section{Resolution of Growth and Maturation}

As collateral vessels grow and mural cells proliferate, there is a corresponding expansion of the media and a formation of a neointima in a disorganized pattern $(32,35)$. This corresponds in time with the expansion of lumenal diameter. However, as lumenal diameter increases and network conductance stabilizes, inflammatory markers start to decrease and angiostatic and anti-inflammatory markers increase (21). Macrophages that have accumulated around collateral vessels also begin to decrease, which parallels a decrease in vascular cell proliferation $(32,45,46)$. Moreover, there is then a reestablishment of a normal balance between ECM breakdown and stabilization that eventually results in a mature basement membrane $(36,38)$. Similarly, as inflammatory and growth signals decline, the smooth muscle cells return to a contractile phenotype $(32,35)$. However, there appear to be two directions that maturating collateral vessels can take. The largest, most developed collateral vessels tend to mature and stabilize, but the smaller, less developed collaterals vessel undergo neointimal hyperplasia and eventual regression $(32,47)$. This is likely determined by network hemodynamics and collateral perfusion pressure, since the inflammatory process and monocytes simply allow for a bidirectional remodeling that is determined by vascular tone and pressure (48). Therefore, 
temporal changes in physical forces, inflammation, growth signaling, and matrix remodeling interact with BMCs and intrinsic vascular cells to coordinate the development of mature collateral vessels. Given the coordinated time-course of the process, it suggests the importance of understanding the sequence of events and cell involvement that occurs during arteriogenesis to understand when, where, and how potential therapies influence each stage of the remodeling process.

\section{Leukocyte Types and Temporal Recruitment In Arteriogenesis}

As early as 1976 (14), it has been shown monocytes play a role in the remodeling process. Since then, a variety of other bone marrow derived cells (BMCs) have also been shown or suggested to mediate arterial remodeling. With the identification of these additional leukocyte populations and subpopulations, there appears to be a complex interrelation between leukocyte recruitment and coordination of the arteriogenesis process. Therefore, there is a need to explore how these various BMC populations influence each other to begin to understand whether certain cell types control important choke points to collateral growth.

Moreover, as these new BMC populations are incorporated into our understanding, it is also important to incorporate the element of timing. Beyond the classic focus on monocyte and macrophages, the proposed roles for other BMC populations must be explored during the various phases of collateral growth. We contend that there is likely to be a coordinated temporal component to the roles for BMCs during collateral development that parallels the phases of the process: initiation, growth, and maturation (Figure 1.2). Thus, the exploration of the timing of recruitment and function in various BMC populations could be particularly important for mechanistic insight into the process and for developing therapeutic strategies that individually target the initiation, growth, or maturation phases of collateral development. 


\section{Neutrophils}

As discussed earlier, during the initiation phase, various chemokines and adhesion molecules are upregulated along the stimulated vessel. Neutrophils (PMNs) are one of the first leukocytes to respond to these types of signals in a variety of inflammatory situations. During these events, PMNs are often recruited from the vasculature within hours $(49,50)$. While there have been few studies on when and where neutrophils are recruited during arteriogenesis, the general pattern of inflammation that occurs in arteriogenesis suggests that neutrophils may play a significant role in the initiation phase. Of the studies that have examined PMN accumulation along collateral vessels, PMNs extensively infiltrate tissues surrounding growing vessels during the initiation phase and their rapid disappearance thereafter (51) suggests a prominent role in commencing collateral growth. This pattern of early neutrophil infiltration within 6 hours followed by monocyte, mast cell, and lymphocyte recruitment agrees with the temporal pattern of cell marker expression observed by Lee et al (21). Moreover, given the short life span of neutrophils (1-2 days after leaving the circulation), these cells likely have a limited role once the large inflammatory stimulus needed for recruitment disappears.

The question remains, however, of what role do neutrophils play during arteriogenesis. While Hoefer et al. have shown that additional neutrophil recruitment does not aid arteriogenesis (52), it does not exclude the possibility that the initial presence of neutrophils significantly contributes to arteriogenesis. Conversely, Ohki et al (53) show that G-CSF administration promotes revascularization through neutrophil-mediated release of VEGF and subsequent progenitor cell mobilization. Moreover, Soehnlein et al (54) recently demonstrated that neutrophil recruitment and secretion products allow for proper recruitment of inflammatory monocytes but have no significant effect on resident monocytes. These results suggest neutrophils may play a similar role in arteriogenesis, helping to regulate the mobilization of monocyte and progenitor cell subpopulations and recruitment to growing collateral vessels. 
Further, neutrophils can play a significant role in vascular remodeling states particularly through the release and production of matrix proteases that alter the microenvironment surrounding vessels $(54,55)$. Nozawa et al $(56)$ recently demonstrated that neutrophils can act as the key mediators of the angiogenic switch in pancreatic tumors through VEGF and MMP secretion. Similarly, Gong et al (57) have shown PMNs play a critical role in corneal angiogenesis. The changes in extracellular matrix can further alter local growth factor gradients (42) and expose cryptic growth signaling sites (40) that promote vascular cell proliferation. Indeed, the loss of neutrophils can lead to reduced VEGF-induced vascular growth (58), stemming from decreased matrix breakdown. Overall, the role of neutrophils in arteriogenesis is still largely undetermined, but evidence suggests a likely role during the initiation phase of collateral growth. The primary impact of neutrophils and natural killer cells during the initiation phase may help explain why adoptive transfer of these cell types do not aid revascularization when administered 24 hours after hindlimb ligation while the administration of monocytes does enhance revascularization (59). It may be that cell types involved primarily during one phase are unable to contribute to collateral growth after that phase has been completed. This type of response highlights the need for understanding the timing and sequence of events during arteriogenesis when devising therapeutic strategies.

\section{Lymphocytes}

Like neutrophils, several lymphocyte populations have been shown to be involved in coordinating arteriogenesis. T-cells were first suggested to influence arteriogenesis in work with athymic mice (60), which showed impaired collateral vessel growth. Stabile et al (61) followed up by demonstrating that CD4 deletion resulted in impaired revascularization after hindlimb ligation due to decreased recruitment of macrophages to growing collateral vessels. This result was corroborated by antibody-mediated depletion of $\mathrm{CD} 4+$ cells leading to impaired arteriogenesis (62). Stabile et al (63) subsequently demonstrated a role for CD8+ cells in 
revascularization. The authors proposed a temporal recruitment pattern of lymphocytes, wherein CD8+ cells are first recruited to the collateral vessel and are then able to influence CD4+ cells and monocyte recruitment through the secretion of $\mathrm{IL}-16$. Together, these studies suggest a recruitment of lymphocytes to collaterals vessels that then influences the recruitment of other BMC populations. However, the concept of lymphocyte-mediated recruitment of monocytes is still unresolved. Despite a decreased capacity to recover from ischemic ligation, athymic nude mice appear to recruit more macrophages than wild-type controls (60). Similarly, the loss of mature $\mathrm{T}$-cells in $\mathrm{MHC}-\mathrm{II}^{-/}$mice results in decreased collateral growth without a significant change in macrophage accumulation in the ischemic tissue (62). This suggests that the role of lymphocytes may be more of a stimulatory role in terms of helping to activate monocytes and propagate monocytes recruitment to enhance the arteriogenic process (64). Additionally, it remains to be seen how and if T-cells contribute to arteriogenesis outside of their effects on other BMC populations.

Also within the lymphoid lineage, natural killer lymphocytes have been implicated in playing a role in collateral vessel growth. However, their actions appear more limited to the initiation phase of the response (62). Two potential roles have been suggested for these cells: tissue clearance to allow for outward remodeling, particularly in constrained tissues such as myocardium (65), and the recruitment of other inflammatory cells (62). Both roles suggest an impact on collateral growth during the early phases of expansion and leukocyte recruitment, but given the uncertain lifespan and trafficking of natural killer cells (66) it is unknown if these cells contribute to any other phases of collateral remodeling.

Furthermore, it is interesting to note that T-cell and monocyte recruitment vary significantly by mouse strain in terms of quantity and timing of recruitment (62). Examination of these differences suggests immune cell recruitment can, in part, explain the spectrum of arteriogenic capacities in mouse strains and humans populations (62) in addition to differences 
in the underlying vascular network $(67,68)$. Moreover, Taherzadeh et al (69) recently demonstrated a similar role for NK cells in the differential vascular remodeling responses to hypertension seen in $\mathrm{BALB} / \mathrm{C}$ and $\mathrm{C} 57 \mathrm{BI} / 6$ strains (the strains most often used to exemplify inter-strain differences in collateral growth). The authors presented evidence that differences between natural killer cell function in BALB/C and C57BI/6 mice contributed to the propensity of the two strains to undergo vascular remodeling, independent from the underlying vascular network structure. Together these findings show a number of roles for lymphocyte populations in coordinating inflammatory cell recruitment and influencing arteriogenesis. These studies suggest an apparent interplay between lymphocytes and other BMC populations in terms of activation and recruitment, which, again, highlight the coordinated nature of collateral growth and the interaction of BMCs with each other to potentiate arteriogenesis. Further, these findings indicate that targeting inflammatory cell function and activity outside of the traditional focus on monocytes may be an effective therapy.

\section{Mast Cells}

Despite an important role for mast cells in other vascular remodeling conditions (e.g. atherosclerosis $(70,71))$ and an initial suggestion of a role in arteriogenesis over a decade ago (35), the role of mast cells in arteriogenesis is largely unexplored. There exists, however, one study on mast cell deficient mice that suggests mast cells do play a significant role in revascularization after hindlimb ischemia (72) with the proposed mechanism centering around mast cell secretion of VEGF and MMP9. Studies on mast cells in other models of vascular remodeling suggest additional likely mechanisms of how these cells are involved in arteriogenesis. First, mast cells secrete a variety of chemokines, cytokine, proteases, and growth factors such as CCL2, GM-CSF, VEGF, PDGF, and bFGF $(71,73)$ known to play a role in arteriogenesis. Second, through the release of these pro-inflammatory products, mast cells appear to play a critical role in coordinating the recruitment of other inflammatory cells from 
initiating neutrophil recruitment (73) to promoting macrophage and CD4+ T-cell accumulation (70). The revascularization study by Heissig et al (72) additionally proposed a mast cell mediated mobilization of progenitor cells from the bone marrow during ischemia that could be enhanced by low-dose radiation. As such, mast cells appear to be involved in arteriogenesis through the recruitment and mobilization of multiple BMC populations and by enhancing vascular cell growth and differentiation through paracrine signaling.

While the timing of mast cell accumulation is poorly understood, the data reported by Wolf et al (35) suggest a larger presence during the initiation and growth phases, with a disappearance as the collateral vessel matures. Since, mast cells are rarely found along collateral vessels in a quiescent state (35), these cells are likely recruited from circulation as basophils. One potential mechanism for this process could be CX3CL1 (fractalkine), which is rapidly (<24 hrs) upregulated upon hindlimb ligation (74) and is known to be a critical mediator of basophil recruitment in vitro (75) and in vivo (76). Given the proposed role in the recruitment of early response cells, such as neutrophils, this fits an early recruitment time course with a sustained presence during the growth phase as paracrine support cells and coordinators of BMC recruitment.

\section{Monocytes}

In contrast, as the most widely studied inflammatory cell involved in arteriogenesis, monocytes have been shown to be critical to the process through multiple methods (77-79). However, even though a role for monocytes has been clearly established, there is now a further need to explore when and where monocytes are recruited and how different subpopulations of monocytes function in collateral development.

The importance of the temporal aspect of monocyte recruitment is emphasized by the mounting evidence that other inflammatory cells help coordinate their recruitment (Figure 1.3). 
In terms of the timing of monocyte recruitment to collateral vessels, it is known that monocytes are particularly prominent in the growing phase of collateral development $(43,46)$. However, the role of monocytes during the initiation of collateral growth is largely unknown. As previously discussed, PMNs are often the first responders to inflammation (50), and in comparison to PMNs, monocytes have a much slower accumulation within collateral tissues after acute occlusion $(21,60,80)$. In other instances of inflammation-induced recruitment of immune cells, such as ischemic injury $(50,81)$, there is a similar process of initial neutrophil predominance that is replaced by macrophages over the course of a few days. As such, macrophages accumulate along collateral vessels within 12 hours after ligation (32) and reach a maximum at around three days but disappear over time $(32,45,46,51)$. This accumulation correlates with vascular cell proliferation. Subsequently, as the collateral vessel begins to mature and proliferative index decreases, the presence of macrophages decreases $(32,45,46)$. The fact that the temporal nature of this recruitment and sustained presence helps elucidate the primary role of monocytes in arteriogenesis belies the importance of understanding the time course of monocyte recruitment. Moreover, increases in circulating macrophage levels after collateral vessels have begun the growth phase do not appear to impact further collateral growth (78). This suggests that macrophages help to potentiate vascular cell growth and structural remodeling during the growth phase but have a decreasing role as these vessels mature. Such a role correlates with a recent study by Bakker et al (48) showing that monocytes act as permissive cells for collateral vessel remodeling with tone dictating the direction of growth (inward vs. outward). However, these data also raise the question of whether an increase in monocyte activity and/or circulating concentration can improve collateral growth if collateral growth is not in the phase where monocyte activity has the greatest impact. Thus, understanding the interplay of timing and recruitment of monocytes by other BMC populations will be critical to determining the exact role of monocytes during arteriogenesis and their optimal therapeutic use. 
Furthermore, many of these studies on the role of macrophages and monocytes use a definition that encompasses a broad spectrum of cell subpopulations. The recent discovery of functional differences between these monocyte subpopulations $(82,83)$ and their varying roles in other types of vascular remodeling (e.g. atherosclerosis $(84,85)$ ) underscores the need to further understand the role of monocyte subpopulations in arteriogenesis. The two most studied of these subpopulations have been termed "resident" ( $\left.\mathrm{Gr}^{1} / \mathrm{LyC} \mathrm{C}^{\mathrm{hi}}\right)$ and "inflammatory $\left(\mathrm{Gr}^{+} / \mathrm{LyC}^{\mathrm{lo}}\right)$ (85). These subpopulations may be identified by a variety of markers, such as relative CCR2 and CX3CR1 expression. These markers have been best characterized in mice but have other mammalian and human analogs (83). A study by Capoccia et al (80) using the adoptive transfer of bone marrow mononuclear cells suggests a functional difference in the capacity of these two monocyte subpopulations to help stimulate collateral growth.

Studies of these characteristic chemokine receptors additionally suggest both monocyte populations have a role in vascular remodeling and arteriogenesis. The most studied chemokine pathway, the CCL2 (MCP-1) and CCR2 axis (34), along with several other studies $(31,80)$ provide ample evidence that inflammatory monocytes $\left(\mathrm{CCR} 2^{\mathrm{hi}} \mathrm{CX} 3 \mathrm{CR} 1^{\text {lo }}\right)$ are critical for coordinating arteriogenesis. The more recent discovery of the resident monocyte subset has prompted one study that also suggests a role for resident monocytes (74). While few if any studies have compared the roles of the two monocyte subsets in arteriogenesis, there is clear evidence showing distinct functional roles in guiding pathologic remodeling in atherosclerosis $(84,85)$. Therefore, based on the observation that atherosclerosis and arteriogenesis share many similar mechanisms (86), it is likely these two monocyte subsets differentially regulation arteriogenesis.

Additionally, the inflammatory and resident subsets exhibit functionally different patterns of recruitment, which can play an important role in vascular remodeling. The inflammatory monocytes $\left(\mathrm{CCR} 2{ }^{\mathrm{hi}} \mathrm{CX} 3 \mathrm{CR} 1^{10}\right)$ reside primarily in the bone marrow and are mobilized from the 
bone marrow and spleen (87) to be recruited to sites of active inflammation through endothelial surface expression of CCL2 ligand (82). In contrast resident monocytes (CCR $2{ }^{\text {lo }} \mathrm{CX} 3 \mathrm{CR} 1^{\mathrm{hi}}$ ) exhibit a patrolling behavior with a large intravascular population that crawls along the venous endothelium, rapidly extravasating to sites of inflammation in a first response like fashion (88). This different pattern of spatial and temporal recruitment results in a differential sensitivity to neutrophil-mediated recruitment of monocytes during acute inflammation (54), where inflammatory monocyte recruitment is decreased with neutrophil depletion. Moreover, in a recent preliminary study in our laboratory, we compared how differentially knocking out the key chemokine receptors for the two subsets (CCR2 and CX3CR1) from bone marrow derived cells effects microvascular remodeling (89). In a model of inflammation-associated arteriogenesis, BMC specific deletion of CCR2 completely abrogated arteriolar remodeling (90) but did not significantly alter venular remodeling (89). In contrast, the loss of CX3CR1 significantly attenuated venular and arteriolar remodeling, though not to the same extent as the loss of CCR2 (89). The different patterns of recruitment between the two subsets results may help explain our results. Taken together, these data provide an example of how functional differences in spatial and temporal recruitment can affect vascular remodeling. As such, it is important to consider the various roles and recruitment patterns of monocyte subsets in arteriogenesis to understand the array of functions monocytes are involved in during collateral development.

\section{Bone Marrow Derived Progenitor Cells}

Since the discovery of endothelial progenitor cells (EPCs) by Asahara et al (91), these cells have stimulated a wealth of studies and have taken a prominent role as a means for treating ischemic disease. Numerous studies have shown EPCs, other bone marrow derived progenitor cells (BMPCs), and induced pluripotent stem cells have the ability to enhance revascularization and take part in vascular repair (91-94). Two key problems, however, have 
limited the ability to translate these laboratory successes to the clinic. First, there is not a definitive set of cell markers for BMPCs or universal isolation techniques, which complicates the interpretation of how these cells influence vascular remodeling and their potential for clinical use $(92,94,95)$. Second, there is a debate, particularly in the arteriogenesis field, of how these cells influence remodeling. Particularly, controversy exists regarding whether BMPCs incorporate into the vasculature and/or act as paracrine signaling sources to support vascular cell growth. This lack of a clear mechanism and difficulty in distinguishing specific cell populations prevents our ability to known where BMPC involvement could have the greatest clinical impact.

Increasing evidence suggests a widely heterogeneous BMPC population (94-96) with at least two functionally distinct sets of EPCs (early and late EPCs (97)), angiogenic T-cells (98), and a variety of other monocyte subpopulations (96). As such, the variety of isolation techniques and surface markers used by various groups have likely pulled unique subpopulations from this complex mix of BMPCs that cannot be directly compared between groups, which has resulted in the large variations of reported therapeutic potential $(92,94)$. Furthermore, each of these various cell populations may play different functional roles during vascular repair, with new functionally different subpopulations arising during ex vivo culture. For example, the neovascularization benefits from the administration of EPCs that arise earlier from colony-forming units during ex vivo expansion (early EPCs) appear to stem from their paracrine support cell role (97), whereby these cells contribute via secretion of angiogenic cytokines and growth factors. Conversely, EPCs arising late in ex vivo culture (late EPCs) appear to function in revascularization by incorporating into activated endothelium and expanding the endothelial cell population (97). These two EPC populations can work together synergistically to promote neovascularization after hindlimb ischemia (95). Similarly, a subpopulation of T cells first identified during EPC colony expansion have the capacity to enhance neovascularization after hindlimb ischemia independent from, and synergistically with, EPCs (98). Additionally, initial studies on the 
adoptive transfer of BM monocytic cells show a marked influence on endogenous leukocyte recruitment, which suggests another mechanism of action (80). All of these studies point to a complex, coordinated interaction of a wide array of bone marrow derived mononuclear cells, especially during ex vivo expansion and reinjection. However, their individual roles and interactions will only be well understood by defining a set of common markers for the various subpopulations and an approach that examines how these cells interact with multiple other cell types.

Multiple groups have demonstrated the potential role for injected BMPCs, after ex vivo culture and/or concentration, to transdifferentiate and directly incorporate into the vascular wall. However, without ex vivo manipulation, the potential for endogenous BMPCs to enhance collateral growth significantly by directly expanding endothelial and/or smooth muscle cell populations appears much less likely. When examined by high resolution confocal microscopy, endogenous bone marrow-derived cells were not seen by Ziegelhoeffer et al (44) to significantly transdifferentiate into endothelial and or smooth muscle cells, attributing the difference from initial reports of extensive incorporation to lower resolution traditional immunohistochemistry techniques. Similar examinations by our group $(99,100)$ and others $(43,59)$ for a variety of vascular remodeling preparations show no evidence that endogenous bone marrow-derived cells can significantly contribute to vascular growth by transdifferentiating and directly expanding endothelial and/or smooth cell populations. Instead, it appears that bone marrow-derived cells contribute to the collateral growth process by localizing to collateral vessels and secreting a variety of paracrine signals to enhance native vascular cell growth and proliferation $(43,101)$. As discussed earlier, BMPCs likely also contribute to the process by coordinating the recruitment of other leukocyte populations.

At this point, we contend there is a need to understand how various BMPC populations influence other leukocytes and to determine how these cells can best be used to enhance 
collateral vessel growth synergistically. Moreover, the discussion of how ex vivo expanded BMPCs versus native BMPCs differ in driving collateral growth through paracrine signaling and/or directly incorporating and expanding vascular cell populations may provide unique strategies for therapeutic stimulation. On one hand, the use of BMPCs to support collateral growth through paracrine signaling can pull on the much larger native vascular cell populations to promote collateral growth during a transient arteriogenic stimulus. However, it has been suggested that endothelial dysfunction can significantly diminish the capacity of an individual to develop collateral vessels (11). In such a case, the use of ex vivo expanded BMPCs for incorporation into the native vasculature may provide a means of providing healthy, nondysfunctional endothelial cells. In either case, there is a substantial need to identify the specific cell populations involved in both processes and the natural time-course for both mechanisms in order to arrive at an optimal therapeutic strategy.

\section{Resident Expansion versus Recruitment of Leukocytes}

The debate over whether macrophages arise primarily from resident sources or from recruitment provides a key example of the need to further understand the temporal and spatial recruitment patterns of leukocyte types. It has been argued that resident macrophages and vascular precursor cells are the primary sources for BMCs that support arteriogenesis $(27,45,102)$. In 2004, Khmelewski et al (45) used cyclophosphamide to deplete circulating monocytes to show that the number of macrophages around collateral vessels increases, despite a $99 \%$ reduction in circulating monocyte counts. While there were limitations to the study that leave room for debating the extent of their role, the results still suggest some role for resident macrophages $(27,103)$. Similarly, fluorescently labeled BMC reconstitutions by our group (100) and Ziegelhoeffer et al (44), show a significant population of BMCs that reside in normal tissue and could serve as ample sources for resident BMCs. Recent studies have suggested these perivascular cells act as a source of mesenchymal progenitor cells (recently 
reviewed by Corselli (104)). However, the primary focus on monocytes neglects the known role of other inflammatory cells that are recruited to collateral vessels such as PMNs and lymphocytes. Given that neutrophils have no resident cell populations and lymphocytes have little capacity to proliferate in extra-lymphoid tissue, there is clear evidence that some populations of circulating inflammatory cells are recruited to collateral vessels. Therefore, it is likely that some populations of monocytes are recruited as well. However, the extent to which resident macrophages contribute to, and how these cells function within, the arteriogenic process is largely unknown. One potential solution to exploring this question may be to differentially ablate resident macrophages or circulating monocytes using CD11b-DTR mice in a method similar to Arnold et al (105).

While there likely is a role for resident macrophage and other support cells that arise from a perivascular pool of previously recruited BMCs, the debate itself centers on a lack of understanding of temporal and spatial recruitment of leukocytes due to the "shear-recruitment paradox". This paradox argues that the physical forces that stimulate collateral growth (i.e. high shear stresses) are less hospitable for leukocyte adhesion. The argument is used as a primary reason for resident cell predominance $(27,45,102)$, because recruitment of leukocytes through the arterial wall would not be able to sufficiently explain leukocyte accumulation around collateral vessels. This logic neglects, however, a key element that collateral arteries are often paired with neighboring veins. Venules may be a significant additional source of leukocyte recruitment that could help explain rapid perivascular accumulation during high arterial shear. Venules are the primary site of leukocyte rolling and adhesion for a wide variety of inflammatory signals, and venules may respond to several of the recruitment signals arising from nearby stimulated arteries (e.g. diffusing chemokines or gaseous products produced in stimulated arterial cells). Communication of diffusible gases and cytokines from venules to arterioles is well established for the regulation of microvascular blood flow (see review by Hester and Hammer 
(106)). Multiple investigators have found that signals released from venular endothelium during elevated shear stress, such as NO, are transferred to paired arterioles and can modulate their behavior $(107,108)$. Similar communication from artery to vein for leukocyte recruitment also appears to exist. For example, activation of arterial endothelium appears to be able to create a signaling gradient for leukocyte recruitment in the neighboring vein through reactive oxygen species (109), which are known to be involved in arteriogenesis (110). Moreover, our lab has recently explored this question of whether ROS can mediate arterio-venous crosstalk in a rat mesentery model of arteriogenesis. We found that ROS induced by high wall shear stresses enhances leukocyte adhesion in both the artery and its paired vein (Song $\mathrm{J}$, unpublished observations). Between the precedence of venulo-arteriolar cross-talk and our recent observations, it would not be surprising to find that the inflammatory and adhesion signals from arteries upon arteriogenic stimulation could be transmitted to paired or neighboring venules and that venules may be a major site of $\mathrm{BMC}$ recruitment during arteriogenesis. However, the question of spatial patterning of leukocyte recruitment during arteriogenesis has been widely avoided, despite the capacity to help provide a solution to the shear-recruitment paradox.

\section{Future Directions}

Given the numerous interactions between leukocyte subtypes and the increased understanding that each subtype may play a particular role within the phases of collateral growth, there is a need to explore, in depth, the recruitment pattern of various leukocyte populations over the time course of arteriogenesis. As such, there is still a place for descriptive studies to examine when various leukocyte populations are recruited to collateral vessels (e.g. flow cytometry studies of isolated collateral vessels, whereby it is possible to screen for numerous leukocyte populations). Such a detailed understanding is particularly important at the early time points where collateral vessels are undergoing rapid changes from quiescent state through an initiation phase to the growth phase of arteriogenesis. Understanding the temporal 
roles of these various leukocyte populations could explain the dynamics of leukocyte interaction and may reveal particular target populations that enhance specific phases of the collateral growth cycle. Moreover, coupled with studies of gene expression at multiple time points through microarray or RNA sequencing of isolated vessels, these techniques have the power to begin teasing apart the molecular signaling pathways of the various collateral growth phases and how bone marrow-derived cells influence the process. Even without such explorations, the emerging evidence on the interactions between the numerous cell populations and signaling events involved in arteriogenesis calls for the reinterpretation of past and future results. It will be important to view these findings through the lens of how alterations in specific pathways during a reductionist approach could affect various other elements in the interconnected arteriogenesis pathway.

\section{Systems and Computational Biology as a New Frontier}

Systems biology, therefore, presents a powerful tool for looking at how the various leukocyte populations and signaling cascades work together to drive arteriogenesis at multiple levels. As highlighted by the recent study by Zbinden et al, gene expression pathway analysis from growing collateral vessels can reveal previously unexplored signaling pathways for stimulating collateral growth $(21,111)$. Similarly, computational network analysis provided an improved understanding of how nitric oxide synthase signaling helps mediate arteriogenesis and collateral vessel network maintenance (112). At a higher tissue scale, earlier modeling of vascular network resistance helped demonstrate the adaptation of the collateral network as the primary means for reperfusion after arterial occlusion $(28,29)$. Moreover, the recent computational modeling in the study by Mac Gabhann et al suggests that only a minimum of collateral pathways need exist for adequate ischemia protection (67), which is corroborated by previous theoretical modeling from de Lussanet (113) and in vivo patient data (114). This computational insight raises the prospect of using targeted delivery of therapeutics for treatment 
without the need of systemic and extensive collateral network restructuring. At a cellular and tissue level, computational modeling could begin to help illuminate how the numerous types of bone marrow-derived cells (including leukocytes and progenitor cells) influence one another and interact with the native vascular cells. The spatial and temporal patterning of leukocyte recruitment, discussed in the previous section, suggest the existence of numerous interactions that could generate rate-limiting choke points in cell recruitment and collateral growth. The identification of such rate-limiting and critical steps in the arteriogenic process could prove invaluable for identifying key therapeutic targets both for the enhancement of collateral growth or for the inhibition of the growth of feeding arteries to tumors. Considering the numerous pathways and feedback interactions that are already known to occur in the remodeling process, the screening of the dynamics of these interactions is best suited to computational modeling approaches followed by targeted in vivo experiments (see review by Sefcik et al (115)). Such insights can further enhance our understanding of the temporal aspects of collateral growth and could identify specific targets for the different phases of arteriogenesis.

\section{Identifying the Common Impairments to Collateral Growth in Patients}

The limited success of large therapeutic arteriogenesis trials despite success in smaller scale trials and subsets of patients may additionally suggest that there are multiple origins of collateral growth impairment and that treatments need to be tailored to specific patient populations $(6,11)$. In particular, there are two questions to answer about the clinical population before moving forward. 1) Are symptomatic patients unable to develop collateral pathways because of an underlying deficit in their endogenous vascular structure and a lack of adequate pre-existing collateral pathways or is there an impairment in their ability to coordinate and undergo arteriogenesis in the collateral vessels that do exist? 2) In patients that do not develop adequate growth from their pre-existing collaterals, is this impairment occurring during the initiation, growth, and/or maturation phases of arteriogenesis? In each patient, there are likely to 
be elements of both answers from both questions. However, understanding the relative importance of these factors through non- or minimally invasive measures of vessel morphology and biomarkers for key elements of arteriogenic phases could provide an invaluable guide for directing patient appropriate therapeutic strategies.

There are also implications for arteriogenesis approaches in terms of the balance between the existence of a pre-existing collateral network versus the ability to develop those pre-existing bridging arteries. Recent studies in mice have demonstrated a wide variation in vascular network structure that lead to collateral-extensive or collateral-sparse networks according to genetic background $(67,68,116)$. These variations have a functional impact on revascularization and recovery after ischemic insult. Such variations likely exist in patient populations. In patients with poor existing collateral networks, it may be necessary to involve a two-fold approach of stimulating the targeted growth of capillary arcades and then stimulating arterialization and collateral growth (e.g. the recent study by Benest et al (117)). One interesting observation from the work by Mac Gabhann et al was that only a relative few number of collaterals need exist to protect against ischemia, which suggests that targeted therapy to establish a limited number of collateral vessels may be all that is needed clinically (67). Conversely, patients with a deficiency in developing collateral vessels may be more responsive to bone marrow derived cell therapy (including leukocytes and progenitor cells), as cell based therapies provide a route for delivering a complex mixture of growth factors and cytokines to coordinate remodeling as compared to individual growth factor treatments (11). Moreover, vascular progenitor cell populations that trend toward incorporating into the endothelial lining may be useful in helping to reestablish normal endothelial function.

However, in order to utilize the full benefits of cell-based therapy, there is a need to consider what elements of the arteriogenesis process are impaired in the patient population of interest. Impairments in each phase of arteriogenic growth may imply a need for different 
approaches for stimulating arteriogenesis in terms of cell types, targeting, and timing of administration. Because the initiation phase is primarily mediated by the endothelial response to increase shear stress along collateral pathways, conditions that lead to endothelial dysfunction (e.g. diabetes (118) and aging (119)) exhibit an impaired arteriogenesis, likely through impaired initiation and network maintenance. This is exemplified by dysfunctional nitric oxide signaling and reactive oxygen species generation resulting in impaired collateral growth that can be restored by normalizing endothelial function and the initiation phase of arteriogenesis $(3,120,121)$. Conversely, aiding growth in later steps through the administration of a growth factor like VEGF may not restore collateral growth (122). Additionally, it is important to consider how decreased endothelial activation could serve to reduce the recruitment of leukocytes that are involved in the initiation and subsequent phases of arteriogenesis. Such disruptions in leukocyte recruitment may explain part of the decreased collateral growth seen in aged mice (119) and nitric oxide synthase knockout mice (23).

Impairments in leukocyte recruitment during the initiation phase may also arise from altered leukocyte migration and function and lead to reduced arteriogenesis. Blocking slow rolling or firm adhesion will substantially reduce collateral growth (33). Similarly, bone marrowspecific knockout of chemokine receptors such as CCR2 can prevent leukocyte recruitment during the initiation phase $(90)$ and impair collateral growth $(90,123)$. Moreover, the decreased collateral growth seen in diabetes mellitus patients can be partially explained by impaired monocyte migration through VEGF chemotaxis (124). Therefore, patients with abnormal initiation of collateral growth may stem from individual or a combination of impairments in endothelial function and BMC function, which may require different therapeutic strategies.

Similarly, patients' coexistent pathologies and inherent capacity for collateral growth can manifest from diminished paracrine signaling function of BMCs, which can affect the growth phase of arteriogenesis. For example, BMCs isolated from patients with coronary artery disease 
have reduced capacity for coordinating revascularization (125) in part from decreased VEGF production in response to hypoxia (126). Likewise, transcriptional profiling of monocyte function by Chittenden et al (127) demonstrated impaired monocyte activation in patients with coronary arterial disease. Genetic lesions in a variety key pathways within BMC populations (e.g. MMP9 in neutrophils (55)) could dramatically alter arteriogenic capacity. These results describe a scenario wherein, even with adequate recruitment, altered BMC function in symptomatic patients may yield reduced collateral growth due to an impaired growth phase of the arteriogenesis process. Further, given the complexity of the recruitment pattern of BMCs to collateral vessels, altered BMC function likely compromises further BMC recruitment and vascular cell activation.

Additionally, while largely unexplored, there are likely elements of impaired maturation and collateral vessel maintenance in certain patient populations. This can be inferred from several recent studies. Recent work from Faber's group suggests that certain genetic factors, such as Clic4 and VEGF-A are required to maintain the collateral connections during embryonic and perinatal development (128-130). Moreover, Dai et al (112) recently demonstrated that nitric oxide signaling is critical to maintaining collateral vessel networks during adulthood. Given the age related declines of nitric oxide production and availability, this may help explain collateral vessel dropout seen with aging (131) and the age-related decreases in collateral growth seen during hindlimb ligation $(112,119,132)$. Collateral growth is also complicated by the fact that there is often preferential pruning of collateral vessels over time as some initially developed collateral vessels undergo stabilization during the maturation phase, while others undergo regression $(2,3)$. Therefore, any arteriogenesis therapies will additionally need to factor in whether the stimuli will be present to maintain or stabilize any newly developed collateral vessels. As such, these findings suggest that there is a need to understand the various factors 
involved that are necessary to promote collateral vessel stabilization and preservation of bridging arteries in the network and how BMCs can influence this process.

When taken as a whole, the evidence suggests that there are combinations of factors that impair collateral growth in symptomatic patient populations. These impairments can occur in each of the different phases of arteriogenesis and can influence BMC function and recruitment at all levels, which, in turn, can influence collateral vessel growth at multiple levels from initiation to growth to maturation and preservation. Each of these factors likely influences the efficacy of various therapeutic strategies in the various patient populations. Therefore, there is a need to define risk factors and biomarkers that correlate with specific impairments in collateral growth in order to select the most efficacious therapies.

Such considerations will be particularly important when evaluating the results of upcoming large-scale arteriogenesis trials using BMCs as the therapeutic agent (e.g BONMOTCLI (133) and (134) JUVENTAS trials). Though initial pilot trials have shown success in treating peripheral arterial disease and coronary arterial disease $(135,136)$, the lack of understanding of individual patient deficits and the coordination of BMCs in arteriogenesis may lessen the potential benefits seen during large scale trials. A similar incomplete understanding of the

coordination of growth factor signaling led to discouraging results in multiple large-scale trials using single growth factors, despite promising results in pilot studies $(6,7,136,137)$.

\section{Conclusions}

Arteriogenesis is a complex process that is highly dependent on proper bone marrow derived cell (i.e. leukocytes and progenitor cells) recruitment and function. The process follows an ordered sequence of events with specific BMC populations recruited at specific times and locations. Many of the actions of these BMC populations are interrelated, influencing endothelial and smooth muscle cell function, as well as the function of other BMCs during subsequent 
phases. Given the complex interactions involved throughout the process, moving forward, it will be important to understand how collateral growth is working as a multi-stage process in relation to BMC activation, recruitment, and function. This further exploration would, ideally, use a twofold investigation into how arteriogenesis is coordinated across multiple elements in normal conditions (i.e. during femoral arterial ligation, where the process is very controlled and has a standard time course) and an examination of pathological conditions to determine where the process is going wrong in the patient population of interest. As such, there is a need to use more "systems-like" approaches where cell population and function interactions throughout the sequence of events in arteriogenesis are considered in parallel during experiment design and interpretation. Such approaches would likely uncover novel bottlenecks for therapeutic targeting and help guide treatment strategies according to the pathological condition of specific patient populations.

\section{Acknowledgements}

The authors would like to acknowledge the contributions and many stimulating conversations with the colleagues in the Price and Skalak laboratories. The unpublished work by the authors referred to in this review was supported by grants from the National Institutes of Health (5R01HL074082 to RJP) and the American Heart Association (09PRE2060385 to JKM/RJP). Additional support came from University of Virginia Center for Undergraduate Education and the American Medical Association Foundation. 


\section{Chapter 1 Figures}

A. Initiation Phase

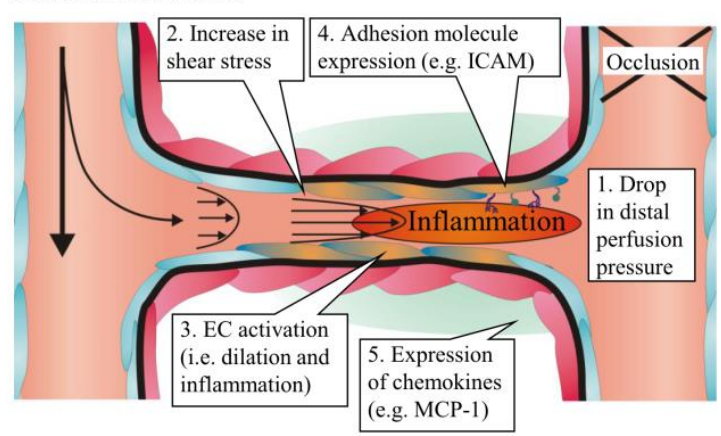

\section{B. BMC Recruitment}

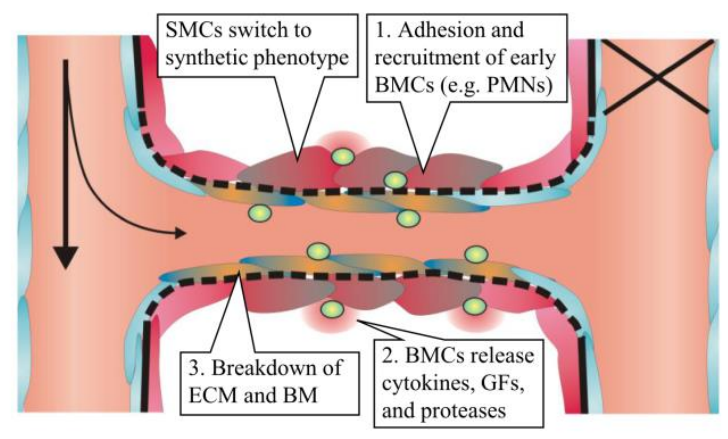

C. Growth Phase

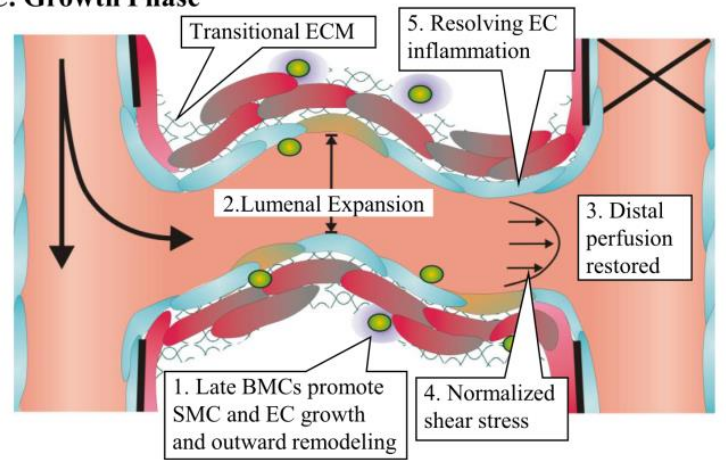

D. Maturation Phase

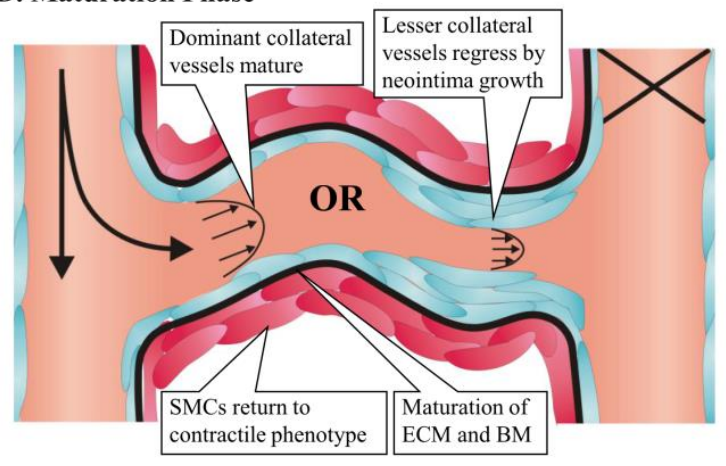

\section{Figure 1.1}

The stages of arteriogenesis can be broken down into three phases (initiation, growth, and maturation). A) The drop in perfusion pressure distal to an occlusion causes increased flow through pre-existing collateral arteries. The increased shear stress triggers an inflammatory response in endothelial cells (ECs, blue, activation indicated by orange) initiating bone marrow derived cell (BMC) recruitment through expression of adhesion molecules and chemokines. B) During the initiation phase, the recruitment of early BMCs (light green) such as neutrophils (PMNs) aid the breakdown of the extracellular matrix (ECM) and basement membrane (BM). Paracrine and autocrine signaling through cytokines and growth factors (GFs) leads to smooth muscle cell (SMC) differentiation into a synthetic phenotype (indicated by grey) to allow for migration, proliferation, and the creation of a transitional matrix. C) Later arriving BMCs (dark green), such as monocytes, further stimulate SMC and EC proliferation and outward growth through paracrine support and matrix remodeling during the growth phase. This leads to lumenal expansion and vessel lengthening in a tortuous pattern, characteristic of growing collateral vessels. As lumenal diameter increases, resistance decreases, distal perfusion is restored, and shear stress begins to normalize. This leads to the resolution of endothelial cell inflammation. D) As inflammation decreases, BMCs disappear and proliferation wanes. Collateral vessels then undergo one of two paths. Vessels with the least resistance supply the most blood flow and mature into the dominant collaterals. Small collateral vessels that cannot maintain sufficient hemodynamic stimulus undergo neointimal hyperplasia and eventual regression. 


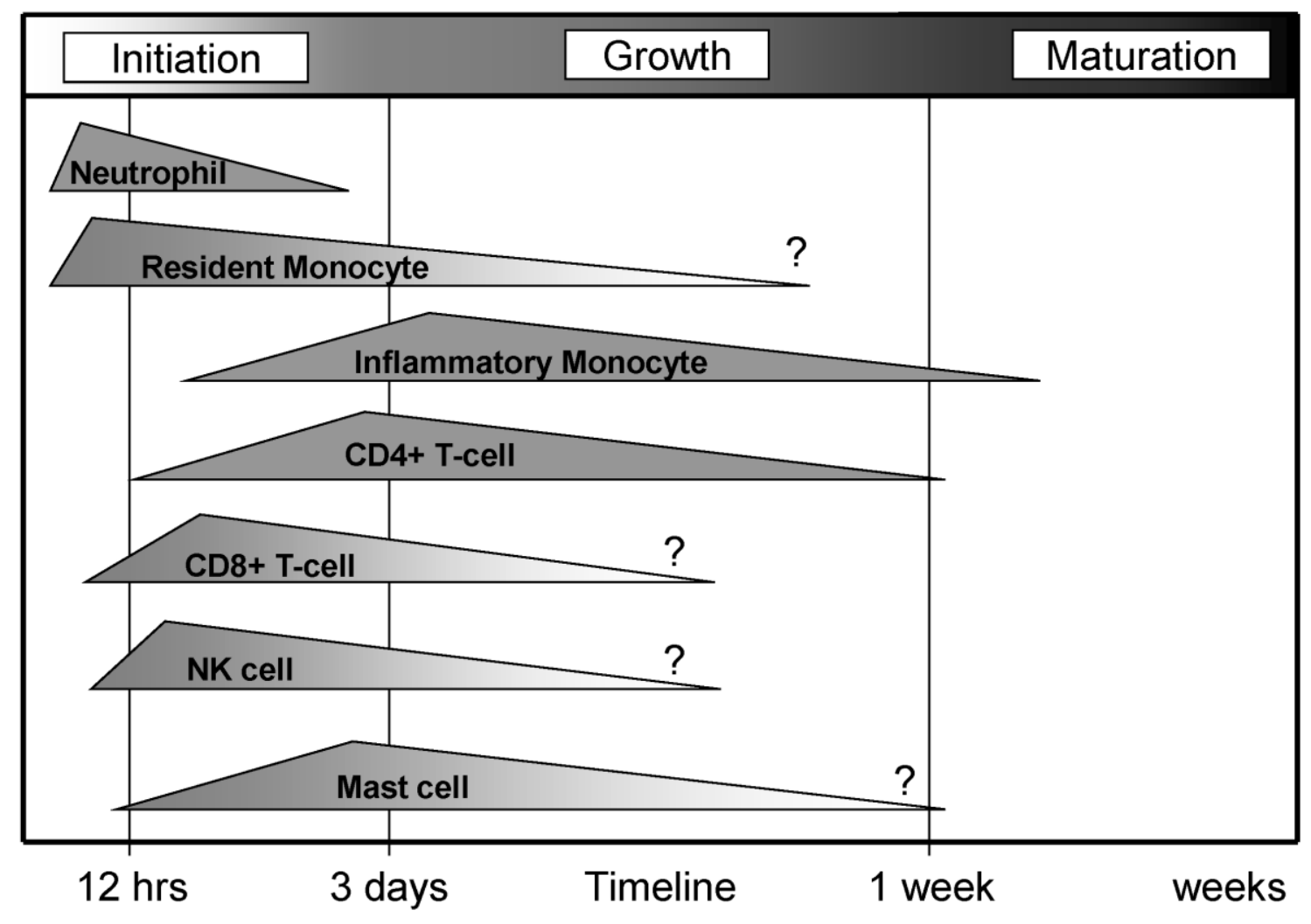

\section{Figure 1.2}

Time line of recruitment of leukocyte subsets during arteriogenesis. The earliest responding leukocytes appear to be neutrophils followed by inflammatory monocytes and mast cells. The temporal recruitment pattern suggests possible relationships between the recruitment of other leukocyte populations. The timing of progenitor cell recruitment, however, is largely unknown. It is important to note that the sequence of leukocyte recruitment and feedback appears to take place within the first few hours and days after the initiation of collateral vessel development. An approximate timeline is given based on BMC recruitment to collateral vessels after femoral arterial ligation. 


\section{Resident}

Monocyte

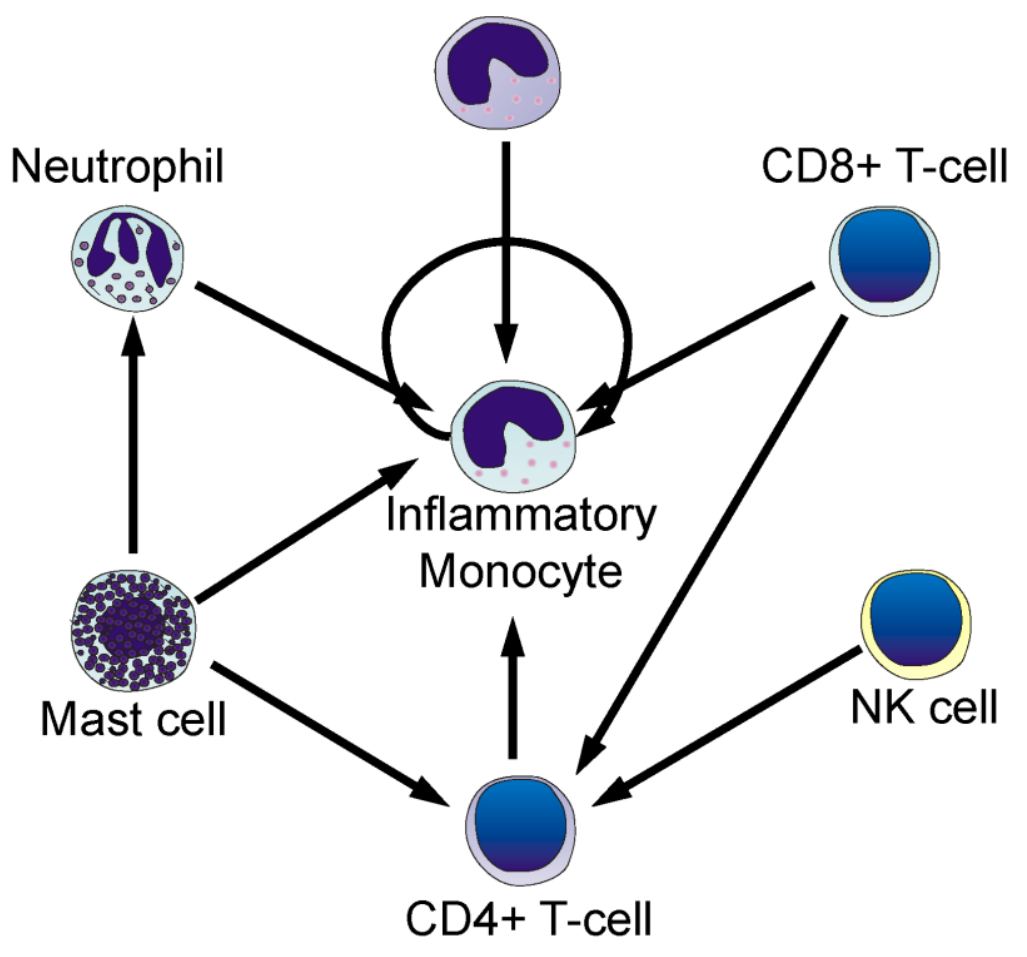

\section{Figure 1.3}

Proposed interconnected relationships of recruitment between BMC populations. Many BMC populations may aid arteriogenesis through the activation and subsequent recruitment of inflammatory monocytes. However, the disruption in earlier BMC recruitment may amplify collateral growth impairments through the reduced recruitment and synergistic activation of other, later-arising BMC populations. How and if bone marrow-derived progenitor cells influence the recruitment of other BMC populations is still largely unknown. 
Chapter 2: Bone Marrow Cell-Derived Matrix Metalloproteinase 9 Deletion Inhibits Reperfusion in the Ischemic Mouse Hindlimb Through Impaired Skeletal Muscle Regeneration, but Does Not Affect Arteriogenesis and Arteriogenesis.

Joshua K. Meisner, Suna Sumer, Ji Song, Sameer S. Bajikar, Brian H. Annex, Richard J. Price

(in preparation) 


\section{Introduction}

Peripheral arterial disease (PAD) is caused mainly by progressive and acute occlusion of the peripheral limb vasculature at atherosclerotic lesions, and it is widely prevalent in the aged population ( $20 \%$ asymptomatic and $6 \%$ symptomatic prevalence in those $>65$ years of age (8)). These ischemic events can ultimately lead to the symptomatic consequences of intermittent claudication and critical limb ischemia (CLI), both significant causes of morbidity and mortality. Within some patients, however, the lack of symptomatic disease can be attributed to their endogenous capacity to compensate to these insults, despite widespread occlusive vascular disease. Thus, one potential treatment for established PAD is to therapeutically stimulate neovascularization in symptomatic patients, where there is insufficient endogenous compensation.

The capacity to endogenously compensate for these ischemic insults comes from a confluence of three processes. First, the pre-existing small arterial pathways that originate upstream of and bypass the occluded region undergo arteriogenesis (i.e. outward remodeling), thereby decreasing upstream resistance and increasing flow downstream (i.e. expansion of inflow). Second, in the tissue downstream of the occluded region, hypoxia induces the expansion of the capillary network (i.e. angiogenesis) to improve the distribution of the blood that does reach the tissue. Clear evidence exists that both adaptive arteriogenesis and angiogenesis can ameliorate PAD, while diseases that impair both arteriogenesis or angiogenesis can contribute to symptomatic PAD $(13,138,139)$. However, there is a third component that is often overlooked: the tissue injured by the ischemia must undergo adaptation and/or regeneration to return functional capacity. In both the disease and in animal models that serve as analogues (i.e. hindlimb ischemia), the ischemic tissue is often the lower limbs. McClung et al. (140) recently provided evidence that suggest previously overlooked differences in skeletal muscle regeneration and response to ischemia may be as important as the more widely studied 
elements of collateral structure and vascular remodeling differences $(116,141,142)$. Ultimately, all three elements must work synergistically to allow for full reperfusion and recovery; however, they are rarely examined in tandem. To date, all phase II or higher clinical trials targeting neovascularization have failed or, at best, been met with marginal success $(4,6,7)$. We postulate that this lack of success stems, at least in part, from an incomplete understanding of how these three elements integrate to lead to functional reperfusion.

Here, we examined the relative roles of arteriogenesis, angiogenesis, and muscle regeneration in establishing functional reperfusion by focusing on matrix metalloproteinase 9 (MMP9), an enzyme that has been hypothesized, in separate studies, to regulate all three processes. Specifically, bone marrow cell (BMC) specific loss of MMP9 is well documented to impair reperfusion capacity in response to an ischemic insult (72,143-146), with BMC derived MMP9 being hypothesized to allow for neovascularization in response to ischemic injury through mobilization of endothelial progenitor cells $(72,146)$ and angiogenesis $(143,145)$. Evidence also exists that BMC-derived MMP9 is involved in arteriogenesis, though the results are conflicting $(48,147)$. In either case, most MMP9 is BMC-derived, with neutrophils being the most prevalent MMP9 cell source $(55,144,148,149)$. However, given that neutrophils have a limited role in the angiogenic $(150)$ and arteriogenic processes $(52,151)$ in response to arterial occlusion, this may suggest the role of MMP9 may lie in regulating skeletal muscle clearance and regeneration. Indeed, MMP9-depedent ECM degradation and remodeling allow for proper satellite cell activation and muscle regeneration during injury (152). One complication in assessing these elements individually is that altered angiogenesis and impaired skeletal muscle regeneration are often correlated and require detailed analysis of both to dissect which is the underlying impairment (150).

Given this evidence, we hypothesized that the primarily role of MMP9 in ischemic muscle injury due to arterial occlusion is not through arteriogenesis and angiogenesis, but instead through 
tissue regeneration. Consistent with this hypothesis and contrary to previous findings $(143,145,146,153)$, we determined that, when accounting for impaired muscle regeneration and clearance, there is no impairment in the dynamics of capillary growth when MMP9 is lost from BMCs. Further, we found that arteriogenesis is not diminished in the primary collateral pathways of the non-ischemic upper hindlimb with loss of BMC derived MMP9. Rather, in mice with BMCspecific MMP9 deletion, impaired reperfusion is best correlated with impaired skeletal muscle regeneration, providing evidence that non-vascular remodeling function may serve as an additional target for PAD revascularization therapies.

\section{Material and Methods}

\section{Animals}

All animal protocols were approved by the Institutional Animal Care and Use Committee at the University of Virginia and conformed to all regulations for animal use outlined in the American Heart Association Guidelines for the Use of Animals in Research. Control (FVB/NJ, strain\# 1800) and MMP9-/ (FVB.Cg-Mmpg ${ }^{\text {tmiTVu} / J, ~ s t r a i n \# ~ 4104) ~ m i c e ~ w e r e ~ p u r c h a s e d ~ f r o m ~ J a c k s o n ~}$ Laboratory (Bar Harbor, ME) and housed in the animal facilities at the University of Virginia. All mice used were male.

\section{Bone Marrow Transplantation}

Bone marrow cell $(B M C)$ transplantations were performed as previously described $(90,99,100)$ to create two groups: $\mathrm{MMP9}^{-1} \rightarrow \mathrm{WT}$ (MMP9 knockout specific to BMC derived cells) and WT $\rightarrow$ WT (control) chimeric mice (denoted as donor $\rightarrow$ host). Recipient mice were irradiated in two doses of 550-600 rads each, 4 hours apart. Bone marrow was harvested from the femur and tibia of each donor mouse (either WT or $\mathrm{MMP9}^{-1}$ ), and approximately 1-2 million unfractionated bone marrow cells suspended in $200 \mu$ of media were delivered through the tail 
vein to reconstitute each irradiated host. Mice were allowed to reconstitute for at least 8 weeks, which we have previously shown yields $>90 \%$ reconstitution (100).

\section{Femoral Arterial Ligation Model}

Femoral arterial ligation (FAL) was performed to induce collateral remodeling in the gracilis adductor muscle and produce a moderate level of ischemia in the downstream tissue in a method similar to that previously described $(99,112,154,155)$. Briefly, age-matched mice $(5-7$ months of age, $>8$ weeks post BMT) were anesthetized (i.p $120 \mathrm{mg} / \mathrm{kg}$ ketamine, $12 \mathrm{mg} / \mathrm{kg}$ xylazine, and $0.08 \mathrm{mg} / \mathrm{kg}$ atropine) and prepped for aseptic surgery. On the left leg, the femoral artery was exposed and isolated from the femoral vein and nerve. Two 6.0 silk sutures were placed immediately distal to the epigastric artery (proximal to the saphenous-popliteal artery bifurcation) and the artery segment between the two ligatures wa severed, and the surgical site was closed. A sham operation, wherein the femoral artery was exposed but not ligated, was performed on the right hindlimb (i.e. on the other leg). Animals received injections of bupernorphine for analgesia immediately post-op and 8-12 hours later.

\section{Micro-Computed Tomography of Mouse Hindlimb}

Micro-computed tomography (microCT) was performed to determine the primary collateral pathways induced with this particular FAL scheme in age and strain matched WT mice. After 14 days, mice were euthanized with an overdose of anesthetic, and the abdominal aorta was cannulated. Lower limbs were then flushed with $2 \%$ heparinized saline and then perfused with saline containing $0.25 \%$ heparin, $0.1 \mathrm{mM}$ papaverine, and $4 \mathrm{mM}$ adenosine to vasodilate the lower limb vasculature. Limbs were then perfused with $4 \%$ paraformaldehyde in PBS and allowed to fix for 30 minutes, followed by perfusion with a radio-opaque vascular casting agent (Microfil@, FlowTech Inc, 2:1:0.15 ratio of color:dilutent:curing agent). Once casting agent was visible in the saphenous artery distal to the ligation site, perfusion was discontinued to limit filling 
only to the primary collaterals, as previously described (155). The casting agent was allowed to cure overnight at $4^{\circ} \mathrm{C}$ followed by decalcification for 5 days. Thigh regions of the limbs were then imaged with a vivaCT40 microCT scanner (SCANCO Medical, Brüttisellen, Switzerland).

\section{Laser Doppler Perfusion Imaging}

Laser Doppler perfusion imaging was performed to monitor blood flow recovery in response to FAL as previously described $(99,154)$. Briefly, mice were anesthetized (i.p $120 \mathrm{mg} / \mathrm{kg}$ ketamine, $12 \mathrm{mg} / \mathrm{kg}$ xylazine, and $0.08 \mathrm{mg} / \mathrm{kg}$ atropine) and placed prone on a surgical heating pad for 5 minutes to minimize temperature variation and allow imaging of the soles of the feet. The lower limbs were scanned (Lisca PIM laser Doppler imager), and mean voltage of foot perfusion was used to calculate relative perfusion ratio (ligated/unligated).

\section{Tissue Harvesting for Cross-sectional Analysis and Collateral Network Structure}

At specified time points $(3,7,10$, and 14 days) after femoral artery ligation, mice were anesthetized, and the gracilis adductor muscle was exposed and vasodilated by drip perfusion of $10^{-4} \mathrm{M}$ adenosine in Ringer's physiologic saline solution. Mice were then euthanized by an overdose of anesthetic and then flushed with $2 \%$ heparinized saline and perfusion fixed with $10 \%$ neutral buffered formalin via cardiac cannulation. After 30 minutes of fixation, gracilis and calf muscles, including the gastrocnemius and plantaris muscles, were harvested and prepared for paraffin embedding or set aside for whole mount immunofluorescence analysis.

\section{Whole Mount Immunofluorescence and Collateral Network Image Analysis}

Un-embedded, formalin-fixed gracilis muscles underwent immunoflourescent staining for smooth muscle alpha actin to visualize the collateral vasculature. Immediately after harvesting, gracilis muscles were washed, and then incubated for 72 hours at $4^{\circ} \mathrm{C}$ in $1: 200$ anti-smooth muscle alpha actin - Cy3 (1A4 clone, Sigma Aldrich, St Louis, MO) in antibody and blocking 
buffer (PBS with $0.1 \%$ saponin, $2 \%$ BSA, and $0.2 \%$ sodium azide). Muscles were then washed and cleared in $50 \%$ glycerol in PBS overnight at $4^{\circ} \mathrm{C}$. Clear tissues were mounted between two coverslips using $500 \mu \mathrm{m}$ thick spacers (645501, Grace Bio-Labs) to keep constant thickness between muscles. Muscles were imaged at $4 \mathrm{x}$ magnification on a Nikon TE300 wide-field, fluorescence microscope with a CCD camera (Microfire, Optronics, Goleta, CA). Individual fields of view were montaged together for network analysis (Photoshop CS2, Adobe Systems Inc). Muscles were then rehydrated and prepared for paraffin embedding.

In each gracilis muscle whole mount montage, there was one primary collateral pathway that spanned from the muscular branch artery to the saphenous artery in each head of the gracilis muscle (see Figure 2.2). Diameter was measured at 9 evenly spaced intervals along the length of the each primary collateral using the Fiji (http://fiji.sc) (156) image analysis platform. The diameter measurements across both heads of the gracilis muscles were pooled to determine mean collateral diameter.

\section{Cross Sectional Analysis of Collateral Structure and Collagen}

Mid-zone sections (5 $\mu \mathrm{m}$ thickness) of paraffin embedded gracilis muscle (cut in half at the midline with both halves mounted) were cut and stained with hematoxylin and eosin (H\&E) for measurements of cross sectional collateral structure or with picrosirius red for analysis of collagen content. In H\&E cross sections (2 sections per leg), the primary collateral artery in each head of the gracilis muscle was identified and imaged at $60 x$ magnification with a color CCD camera (Microfire, Optronics, Goleta, CA). Wall thickness and wall nuclei measurements were determined from manual tracing and counting using the Fiji image analysis software, where inner and outer diameter was calculated from the traced perimeter.

For collagen analysis, the primary collateral artery in each head of the gracilis muscle was identified and imaged in two sections under brightfield and circular polarized light (Olympus 
BX51) at 40x magnification. Collagen intensity and area were determined using a custom-built semi-automatic analysis program within the Fiji platform. Briefly, a 230 pixel (10 micron) region around the traced lumenal perimeter was calculated and the corresponding polarized light image underwent automated thresholding to determine collagen birefringence intensity and total collagenous area, which were then normalized to the perimeter.

\section{Immunohistochemistry Analysis of Immune Cell Recruitment}

Sections ( $5 \mu \mathrm{m}$ thickness) of paraffin embedded muscle from the gracilis muscle were labeled by immunohistochemistry for common leukocyte antigen (CD45) for measurement of peri-collateral recruitment of inflammatory cells or were stained using toluidine blue to identify peri-collateral mast cells. For CD45 cell staining, slides were dewaxed, rehydrated, and underwent heat mediated antigen retrieval (0.5 min at 22 psi and $125^{\circ} \mathrm{C}$, DAKO Pascal Pressure Chamber) using DAKO TRS citrate-based buffer. Slides were then blocked and labeled with CD45 primary antibody (1:80, 550539, 30-F11 clone, BDBiosciences) followed by a biotinylated goat anti-rat $(1: 200)$ secondary and streptavidin-HRP (1:200). Chromatic coloring was developed using DAKO DAB+ substrate and counterstained with hematoxylin. The primary collateral artery in each head of the gracilis muscle was identified in 2 sections per slide and was then imaged at 60x magnification with a color CCD camera (Microfire, Optronics). CD45-positive or toluidine blue positive cells within $\sim 25 \mu \mathrm{m}$ from the artery were counted using Fiji image analysis software.

\section{Cross Section Immunofluorescence}

Sections (5 $\mu \mathrm{m}$ thickness) of paraffin embedded muscle from the gracilis and calf muscles were labeled for multi-color immunofluorescence for visualization of CD31 (PECAM1), MMP9, and macrophages (Mac3). For all antigens, slides were dewaxed, rehydrated, and underwent heat mediated antigen retrieval in a 1200W microwave (10 min for MMP9/Mac3, 20 min for CD31) in 
citrate-based antigen retrieval buffer (Vector Laboratories, H-3300). Slides were then blocked and labeled with primary antibody [rat anti-CD31 (1:100, SZ31 clone, HistoBioTec LLC, Miami Beach, FL), rabbit anti-MMP9 (1:500, ab38898, Abcam), rat anti-Mac3 (1:50, M3/4 clone, 550292, BD Biosciences)] overnight at $4^{\circ} \mathrm{C}$. Slides were then washed and incubated with goatanti rat Alexa Fluor 647 (1:200, Life Technologies) and/or goat anti-rabbit Alexa Fluor 555 (1:200, Life Technologies) for 45 minutes at room temperature for CD31 and MMP9, respectively. Mac3 required 3 step detection using a biotinylated sheep anti-rat (1:200, Abcam) secondary followed by streptavidin-Alexa Fluor 647 (1:200, Life Technologies). Nuclei on all sections were counterstained using 10nM SYTOX green (Life Technologies). Slides were then sealed with Prolong Gold (Life Technologies) to minimize photo-bleaching.

\section{Cross Sectional Damage Analysis}

Sections (5 $5 \mathrm{~m}$ thickness) of paraffin embedded calf muscles, containing gastrocnemius and plantaris muscles, (cut in half at the thickest region with both halves mounted) were cut and stained with $\mathrm{H} \& \mathrm{E}$ for measurements of skeletal muscle damage. A high resolution image of the entire muscle cross section was reconstructed by stitching together individual fields of view taken at 10x magnification. In the event of partial cross section, the section was excluded from analysis. At least one of two sections was usable in all harvested muscles. Tissue composition was classified into five categories defined as followed: 1) necrotic, where fibers lacked nuclei, were rounded and dilated in appearance, and had weak eosiniphilic cytoplasm; 2) early regenerating, where fibers were small, loosely organized, and had centrally located nuclei; 3) late regenerating, where fibers were approximately the size of mature fibers within the given region, compactly organized, but still maintained centrally located nuclei; 4) fibro-adipose tissue, where there was minimal presence of myoblasts and was dominated by fibrous matrix and adipose tissue; 5) mature fibers, where muscle fibers were comparable in size, organization, and structure for the respective region in unligated control limb. Individual regions of each 
category were manually identified and measured for calculating percent area. Total damaged area was calculated as the sum of regions 1-4.

\section{Capillary Growth (Angiogenesis) Analysis}

Calf muscle cross sections stained for CD31 were used to determine capillary density metrics. Because there are at two largely distinctive regions of the sectioned muscles with significantly different capillary and muscle fiber composition, analysis was separated into two regions: the superficial region within the white gastrocnemius muscle and the deep region containing the plantaris and red gastrocnemius muscles, termed here as the glycolytic and oxidative regions, respectively (157-159). Two fields of view from each region in each section were imaged at $20 x$ magnification on a Nikon TE2000 C1 laser scanning confocal microscope. Capillary density was then determined using a semi-automated image analysis algorithm in Fiji by thresholding for percentage CD31-positive tissue area within a classified region (either fibro-adipose, regenerating, or mature).Capillary to muscle fiber ratio was also calculated to account for changes in muscle fiber size. Specifically, capillary and viable (regenerating or mature) muscle fibers (identified by auto-fluorescence) were counted within each field of view, yielding $>100$ and >200 muscle fibers per glycolytic or oxidative region per muscle, respectively. Capillary to muscle fiber ratio was also determined in damaged, but viable, oxidative regions of day 3 postFAL limbs that showed intermixing of necrotic and viable muscle fibers as determined by morphology in H\&E stained serial cross sections.

For comparison to laser Doppler perfusion measurements collected at the day of harvest, vascular area per total muscle fiber cross section was approximated by using the mean CD31+ area across each type (mature, regenerating, fibro-adipose, and necrotic) averaged across muscle region and mouse groups multiplied by the percentage of the tissue types within each cross section. Necrotic vascular density was calculated as zero. 


\section{Statistics}

All results are reported as \pm standard error of the mean. All images were randomized and deidentified to enable blinded analysis. All data were first tested for normality. Statistical significance was assessed by one- and two-way ANOVA, followed by paired comparisons using the Holm-Sidak method for multiple comparisons (SigmaStat 3.5, Systat Inc). Co-variance with laser Doppler perfusion measurements was assessed by Spearman's correlation. Significance was assessed at $\mathrm{P}<0.05$.

\section{Results}

\section{Loss of BMC-Derived MMP9 Impairs Perfusion Recovery After FAL}

Laser Doppler Perfusion Imaging (LDPI) was used to evaluate how the loss of MMP9 from BMCs affects perfusion recovery in response to FAL (Figure 2.1). BMP-specific MMP9 deletion inhibited perfusion recovery, with the strongest difference occurring at 4 days post ligation. This particular FAL model showed a moderate level of ischemic impairment, with a plateau in recovery around day 10 post-FAL, but did not return to baseline levels of perfusion.

\section{Loss of BMC-Derived MMP9 Does Not Alter Arteriogenesis in Response to FAL}

Whole mount and cross sectional analysis of the collateral network in the gracilis muscle was used to assess arteriogenesis in the non-ischemic thigh region (160). Micro-CT angiography was used to determine the primary collateral pathways using this FAL model in the FVB/NJ strain. Similar to the C57BL/6 strain $(112,155,160)$, all mice showed prominent growth and characteristic tortuosity indicative of arteriogenesis in the collateral arteries of the gracilis adductor muscle (Figure 2.2A, $\mathrm{n}=4$ ). Whole mount analysis of collateral arteries labeled with smooth muscle alpha actin (Figure 2.2B) was used to quantify the degree of arteriogenesis with or without BMC-specific loss of MMP9. In both chimeric mouse groups, there was significant 
remodeling of the arteries within the ligated limb compared to the unligated control limb. The degree of outward remodeling increased with time, but stabilized after 7 days post-FAL (Figure 2.2C). However, there was little variation in the degree of outward remodeling between the chimeric groups. At day 7 post-FAL, there was a slight, but significant increase in outer diameter within the ligated limbs of the $\mathrm{MMP}^{-/} \rightarrow \mathrm{WT}$ mice compared to WT $\rightarrow \mathrm{WT}$ controls, but this difference disappeared at later time points (Figure 2.2C). There were no significant differences in baseline collateral diameter between the chimeric groups.

Cross-sectional analysis of collateral growth at the mid-zone of the gracilis muscle showed that the outward remodeling seen in the immunofluorescence-labeled whole mounts was matched with a functional increase in wall mass and wall nuclei (Figure 2.2D and 2.2E). By day 7 post-FAL, there was a significant increase in wall thickness (Figure 2.2E) and mural cell nuclei per vessel (Figure 2.2E) that was present at all subsequent time points. Similar to the collateral network analysis seen in the muscle whole mounts, there were no significant differences in wall thickness or mural cell number to indicate differential remodeling between chimeric groups within either the ligated or unligated limbs, including day 7 post-FAL.

\section{Loss of BMC-Derived MMP9 Does Not Alter Peri-Collateral Collagen Remodeling in Response to FAL}

Additional cross sectional analysis of collateral arteries in the mid-zone of the gracilis muscle was used to determine if loss of BMC derived MMP9 altered collagen degradation during arteriogenesis. By immunofluorescence, MMP9 was not detected in peri-collateral cells recruited during arteriogenesis (Figure 2.3A, supplement Figure 2.S1). Further, macrophages, which are known to play a key role in arteriogenesis (2), were similarly present around collateral arteries in the ligated limb in both WT $\rightarrow$ WT and $\mathrm{MMPg}^{-/-} \rightarrow \mathrm{WT}$ mice (Figure 2.3A). BMC-derived MMP9 (type IV collagenase) involvement in arteriogenesis was further characterized by 
quantification of collagen content in the ligated and unligated limb through picrosirius red staining and birefringence to circularly polarized light (Figure 2.3B). Both peri-collateral collagen intensity (Figure 2.3C) and total peri-collateral collagen area (Figure 2.3D) were decreased by day 7 post-FAL around the remodeling collateral arteries within the ligated limb within both $\mathrm{MMP}^{-/-} \rightarrow$ WT and WT $\rightarrow$ WT groups, but there were no significant differences between MMP9 ${ }^{-/-}$ $\rightarrow \mathrm{WT}$ and WT $\rightarrow \mathrm{WT}$ mice within the remodeling or control (unligated) collateral arteries.

\section{Loss of BMC-Derived MMP9 Does Not Alter Leukocyte or Mast Cell Recruitment}

We assessed total leukocyte recruitment by CD45 positivity and mast cell density (previously suggested to be involved in MMP9-mediated reperfusion (72)) by toluidine blue staining in the mid-zone of the gracilis muscle (Figure 2.4A). As expected, there was significant recruitment of leukocytes to remodeling collateral arteries within the ligated limb at all time-points (Figure 2.4B). However, there were no significant differences in pericollateral leukocyte numbers between $\mathrm{MMPg}^{-/} \rightarrow \mathrm{WT}$ and WT $\rightarrow \mathrm{WT}$ groups in either the baseline (unligated) or remodeling (ligated) conditions. While present in a pericollateral position at a low density, similar to CD45+ leukocytes at baseline, there was no additional increase in toluidine blue positive cells in the remodeling collateral arteries (Figure $2.4 \mathrm{C}$ ). No significant differences between $\mathrm{MMP}^{-/} \rightarrow \mathrm{WT}$ and WT $\rightarrow$ WT groups were present in either the baseline (unligated) or remodeling (ligated) conditions.

\section{Loss of BMC-Derived MMP9 Does Not Alter Angiogenesis in Response to FAL}

To determine if the impaired perfusion recovery seen in $\mathrm{MMPg}^{-/} \rightarrow \mathrm{WT}$ mice was related to altered capillary remodeling in the downstream ischemic tissue, BMC recruitment and capillary growth (i.e. angiogenesis) were assessed in the ischemic calf muscles. Previous findings suggest the highest degree of MMP9 expression seen in ischemic muscle occurs within the first few days of ischemia $(40,144,161)$. Therefore, day 3 post-FAL was chosen for assessment of 
BMC recruitment and MMP9 production. Only ischemic regions with high inflammatory cell infiltrates showed prominent MMP9-positive cell staining (Figure 2.5). As expected, MMP9-/ $\rightarrow$ WT showed negligible MMP9-postive cell staining, constituting a >95\% reduction in MMP9positive cells within infiltrated ischemic tissues at day 3 post-FAL $(2.4 \pm 1.1$ vs $84.2 \pm 16.2$ MMP9+ cells/high power field for $\mathrm{MMPg}^{-/-} \rightarrow$ WT vs WT $\rightarrow$ WT mice, respectively, $\mathrm{n}=5$ mice/group) (Figure 2.5). The infiltrating cells in this tissue predominantly represented macrophages as indicated by Mac3-positive staining, but macrophages were not prominent sources of MMP9 production (Figure 2.5, top row). Both $\mathrm{MMPg}^{-1-} \rightarrow \mathrm{WT}$ and WT $\rightarrow$ WT groups showed a similar degree of cellular infiltration within necrotic regions of the muscle at day 3 post-FAL (71.5 \pm $3.6 \%$ vs $61.6 \pm 12.4 \%$ infiltrated area of necrotic fibers for $\mathrm{MMPg}^{-1-} \rightarrow \mathrm{WT}$ vs WT $\rightarrow$ WT mice, respectively, assessed from complete H\&E stained calf muscle cross sections $n=5$ mice/group, $\mathrm{p}=0.37)$.

The spatial variation in muscle fiber type and capillary structure within the calf muscles (157-159) necessitated a separate analysis of the glycolytic (superficial white gastrocnemius muscle) and oxidative (plantaris and deep gastrocnemius muscle) regions of the muscle for assessment of capillary growth. Similarly, the ischemia induced by FAL produced non-uniform damage throughout the muscle, which also required separation of vascular growth analysis (supplemental Figure 2.S2). Regions were separated into four types of tissue and vascular structure, based on histological morphology: necrotic tissue, mature muscle fibers, regenerating muscle fibers, and fibro-adipose tissue (Figure 2.6A). As demonstrated in Figure 2.6A, necrotic regions completely lacked CD31+ vascular structures. Mature fibers showed the typical organized capillary structure present in skeletal muscle. Regenerating fibers showed a similarly organized capillary network between developing fibers, but with a higher density than mature tissue. Fibro-adipose tissue, however, showed largely unorganized structure with heterogeneous CD31+ vascular tissue. When CD31+ vascular area (proportional to a 
measurement of capillary density) was quantified per vessel region within the ischemic tissue at 14 days post-FAL, there were broad differences in each region (Figure 2.6B). Specifically, the vascular area of fibro-adipose tissue>regenerating fiber tissue>mature fiber tissue (Figure 2.6B). However, the variation in capillary structure between oxidative and glycolytic regions seen at baseline was lost (Figure 2.6B, 2.6C). Because the necrotic regions were completely avascular, they were excluded from analysis for comparison of total CD31+ tissue area. As expected, the ischemic tissue showed a greater density of CD31+ vascular tissue in total than the baseline (unligated) state within both glycolytic and oxidative regions (Figure 2.6C). However, there were no significant differences in vascular area between MMP9 ${ }^{-/-} \rightarrow$ WT and WT $\rightarrow$ WT groups in either baseline or ischemic tissue in either muscle region (Figure 2.6C).

As a second metric to better define the dynamics of vascular growth within the ischemic muscle tissue after 14 days post-FAL, capillary to muscle fiber ratio and capillary density were measured within regions of viable muscle fibers (i.e. regenerating or mature muscle). As expected, capillary density (Figure 2.6D), which is proportional to the CD31+ vascular area measurements, showed a similar relationship to the previous CD31+ vascular area results. There was a significant increase in capillary density in the ischemic muscle versus the nonischemic muscle in both $\mathrm{MMPg}^{-/-} \rightarrow \mathrm{WT}$ and WT $\rightarrow \mathrm{WT}$ mice in the glycolytic and oxidative regions. While there were no significant differences in capillary density in the non-ischemic muscle between $\mathrm{MMPg}^{--} \rightarrow \mathrm{WT}$ and WT $\rightarrow \mathrm{WT}$ mice, there was a slight, but significant, increase in capillary density in the ischemic muscle of $\mathrm{MMPg}^{-/} \rightarrow \mathrm{WT}$ mice versus the WT $\rightarrow \mathrm{WT}$ mice within the glycolytic muscle region. However, when the change in muscle fiber size was accounted for using the capillary to muscle fiber ratio, there were, again, no differences between $\mathrm{MMPg}^{--} \rightarrow$ WT and WT $\rightarrow$ WT groups within non-ischemic or ischemic muscle in either glycolytic or oxidative regions of the muscle (Figure 2.6E). Further, when the change in muscle fiber size was taken into account, there was an increase in capillarity, indicative of angiogenesis, within 
the glycolytic region, but a decrease in capillarity in the oxidative region (Figure 2.6E). Additional capillary density and capillary to muscle fiber ratio analysis was performed at the day 3 postFAL time point to determine if BMC-specific loss of MMP9 altered capillary loss within the ischemic tissue. Similar to the day 14 time point, there were no significant differences in capillary density or capillary to muscle fiber ratio (Figure $2.6 \mathrm{~F}, 2.6 \mathrm{G}$ ).

\section{Loss of BMC-Derived MMP9 Impairs Ischemic Skeletal Muscle Recovery after FAL}

To determine if altered skeletal muscle recovery could account for the decreased reperfusion within $\mathrm{MMPg}^{-/} \rightarrow \mathrm{WT}$ mice, the composition of the muscle tissue within the damaged tissue regions was determined by histological analysis (Figure 2.7). Unlike collateral growth or capillary density measurements, there was a significant impairment in the dynamics of skeletal muscle recovery within the $\mathrm{MMPg}^{-1-} \rightarrow \mathrm{WT}$ mice versus the WT $\rightarrow \mathrm{WT}$ mice (Figure 2.7B, 2.7C). The degree of regrowth of regenerating muscle fibers (early and late stage) within the damaged tissue region was significantly impaired in the $\mathrm{MMPg}^{-/} \rightarrow \mathrm{WT}$ mice versus the WT $\rightarrow$ WT (Figure 2.7C). These impairments were most prominent at day 10 post-FAL, where $41.1 \pm 12.2 \%$ of the damaged tissue had already begun regenerating muscle fibers within the damaged tissue in WT $\rightarrow$ WT mice versus only $7.9 \pm 2.2 \%$ within $\mathrm{MMPg}^{-1-} \rightarrow \mathrm{WT}$ mice (Figure $\left.2.7 \mathrm{C}\right)(\mathrm{p}=0.003, \mathrm{n}=7$ and 6, respectively).

\section{Impaired Perfusion Recovery is Associated With Impaired Ischemic Skeletal Muscle Recovery}

Comparisons of vascular area, mean collateral diameter, and degree of muscle fiber damage to the corresponding laser Doppler L/R perfusion measurements on the day of harvest were used to assess how each of these elements co-varied with hindlimb perfusion. Surprising, neither approximate CD31+ vascular area nor mean collateral diameter show correlation with the corresponding perfusion measurements by laser Doppler perfusion imaging (Figure 2.8F and 
2.8D, $p=0.536$ and 0.416 , respectively). However, the percentage of non-viable muscle tissue (fibro-adipose and necrotic fiber regions) showed significant correlation with their corresponding perfusion measurements $(p<0.001$, Figure 2.8B).

\section{Discussion}

\section{Summary}

We report here that impaired reperfusion in response to $F A L$ in mice with BMC-specific MMP9 deletion is mediated by impaired skeletal muscle regeneration. We base this central conclusion on three key findings. First, the BMC-specific loss of MMP9 had no detrimental effect on FAL-induced arteriogenic remodeling of the primary collateral arteries in the upper hindlimb (Figure 2.2). Second, when differences in tissue composition were taken into account, we observed no differences in capillary growth within the ischemic tissue of $\mathrm{MMP}^{-/-} \rightarrow \mathrm{WT}$ mice when compared to WT $\rightarrow$ WT controls (Figure 2.6). Third, the loss of BMC-derived MMP9 did, however, significantly mar skeletal muscle repair within the distal ischemic tissues (Figure 2.7). This impairment of skeletal muscle regeneration showed the strongest correlation with diminished reperfusion (Figure 2.8). Together, these data suggest that studying arteriogenesis, angiogenesis, and tissue regeneration can provide better insight into the mechanisms of the reperfusion response to ischemia. Furthermore, they indicate that, in addition to stimulating collateral arteriogenesis and/or ischemic tissue angiogenesis, therapeutic approaches for stimulating reperfusion in PAD may stand to benefit significantly by targeting improved skeletal muscle regeneration.

\section{Comparison to previous studies and the need for detailed assessment of angiogenesis and arteriogenesis}

Multiple studies have shown decreased revascularization with loss of MMP9 in the context of hindlimb ischemia models, with the impairment being primarily attributed to BMC- 
derived MMP9 $(72,143,145,146)$. These studies have, however, largely focused on the role of angiogenic remodeling, independent of detailed consideration of arteriogenesis and/or skeletal muscle regeneration, which effects how the role of MMP9 during revascularization is interpreted. For example, a substantial increase in tissue necrosis is seen in $\mathrm{MMP9}^{-/-}$mice $(72,145,146)$, which could skew the estimates of angiogenic metrics, such as capillary density and capillary to fiber ratio if not adequately compensated for by exclusion of necrotic fibers (162). In turn, this can lead to capillary to fiber ratios significantly below those expected for viable muscle $(145,146,162)$. Further, even within normal and regenerating tissue (i.e. nonnecrotic) tissue, alterations in cross-sectional muscle fiber size can affect capillary density (162), making the capillary density measurements difficult to interpret without corresponding assessment of muscle fiber cross sectional area (143). Here, we demonstrate that, through detailed analyses to account for these potentially confounding factors, there is no shift in capillary to muscle fiber ratio or capillary density within tissue-type regions, thereby suggesting no angiogenic impairment with loss of BMC-derived MMP9. Nonetheless, while these additional considerations may impact how results from previous studies are interpreted, there is still strong evidence that loss of MMP9 significantly impairs reperfusion capacity and that this is linked specifically to altered BMC function $(143,145,146)$. These previous findings are clearly supported by the current study (Figure 2.1).

Another possible explanation for diminished reperfusion capacity in $\mathrm{MMPg}^{-/} \rightarrow \mathrm{WT}$ mice was deficient upper hindlimb collateral arteriogenesis. Therefore, a detailed examination of arteriogenic growth was similarly necessary. This analysis was facilitated by the careful selection of the FAL model used. While the FAL model used here does not demonstrate the same degree of ischemia as the more widely used femoral artery excision models $(155,163)$, it does yield consistent remodeling of the gracilis muscle collateral pathways. Such an approach has been previously useful to confirm or exclude the involvement of arteriogenesis in the 
reperfusion response $(112,155,160)$. Here, we showed that, unexpectedly, MMP9 ${ }^{-/} \rightarrow W T$ mice exhibit fully functional arteriogenesis. When considered in light of the angiogenesis data, this leads to the conclusion that the impaired reperfusion response observed in mice with loss of BMC-derived MMP9 is not due to defective vascular growth and remodeling. Together these results argue for the careful selection of the ischemic model and subsequent detailed analyses of both arteriogenesis and angiogenesis when investigating the mechanisms of, and treatments for stimulating, ischemic tissue reperfusion.

\section{Potential Mechanisms of MMP9 in impaired muscle repair}

A critical question that arises from our current data is that of how BMC specific loss of MMP9 impairs skeletal muscle regeneration. At first, there does not appear to be a direct connection because neutrophils, which are the primary source of MMP9 in ischemic muscle $(55,144,149)$, are not prominent contributors to reperfusion $(52,151)$ and may actually inhibit skeletal muscle repair during ischemia (164). However, there are multiple lines of evidence suggesting that MMP9 can play a role in skeletal muscle repair through both degradation and clearance of necrotic tissue and/or through activation and migration of satellite cells $(152,165,166)$. While some of the MMP9 involved in satellite cell activation may be of satellite cell origin (167), our data still suggests a prominent role for BMCs and MMP9 of BMC origin in full BMC function. With respect to ECM degradation (166), the time course of this process coincides with MMP9 expression during hindlimb ischemia, which peaks at the initial few days during the clearance of necrotic tissue by myeloid cells (164) and then begins to taper off before the appearance of regenerating fibers $(40,161)$. During this phase, MMP9 is known to be involved in the release of growth factors during ECM breakdown (168) and in the exposure of cryptic epitopes (40) such that, in combination with angiogenic growth factors, MMP9 can promote balanced muscle and vascular growth during ischemia $(41,169,170)$. An additional explanation may be that the impairment of endothelial progenitor cell recruitment and 
mobilization during hindlimb ischemia seen with BMC-specific loss of MMP9 $(55,145,146)$ may directly contribute to the impairment in skeletal muscle repair. If not involved in altered angiogenesis dynamics, the loss of EPC recruitment or altered function may still inhibit muscle repair through loss of paracrine growth signaling. This could occur through mechanisms such as MMP9 mediated activation of IGF1 (171) or modulation of recruited monocytes and macrophages toward more reparative phenotypes, a recently identified primary role for multipotent adult progenitor cells (172).

\section{Importance of skeletal muscle regeneration in vascular function}

Another interesting facet of our study is that, while not directly tested, our data suggest that different tissue compositions (i.e. normal, regenerating, necrotic, and fibro-adipose) have different capacities for enabling reperfusion because, at least in the context of this animal model, microvascular network topology, morphology, and density are intrinsically linked to tissue composition. This is not surprising given that several recent studies have shown that treatments that expand microvascular networks without maintaining normal topologies and/or dimensions do not necessarily improve-and may even decrease-reperfusion in response to ischemia because they are hemodynamically inefficient (173-175). This may explain the paradoxical result that poor reperfusion was observed in mice with a large composition of highly vascularized fibro-adipose tissue. Therefore, we contend that the promotion of proper skeletal muscle regeneration in combination with the inhibition of fibrosis and adipogenesis in ischemic muscle may yield disproportionate, yet more effective, reperfusion.

Lastly, it is important to consider that, clinically, MMP9 has been primarily proposed as a biomarker for PAD, with increasing MMP9 production linked to increasing disease severity $(41,149,176)$. This seems counterintuitive given our results and previous evidence that loss of MMP9 decreases revascularization potential. However, MMP9 expression seems to peak during 
the initial ischemic insult and recede upon perfusion recovery $(40,144,161,165)$, which suggests that MMP9 expression and production are enhanced during active ischemia. Therefore, we postulate that the MMP9 biomarker may be more indicative of increasing ischemic muscle injury, which correlates with increasing severity of PAD. As such, rather than altering the interpretation of MMP9 as a biomarker for PAD, the potential clinical insight seen here is the demonstration that altered tissue skeletal muscle repair alone can have a significant impact on tissue reperfusion. This implies that variations in skeletal muscle regeneration capacity may play a role in determining symptomatic and progressive PAD within certain patient populations. Further, these data suggest that strategies targeting skeletal muscle regeneration in conjunction with targets to enhance vascular remodeling may yield synergistic effects for therapeutic revascularization approaches toward treating PAD.

Acknowledgements: The authors would like to thank the University of Virginia Biorepository and Tissue Research Facility and the University of Virginia Research Histology Core for their work in histological tissue processing.

Sources of Funding: This work was supported by American Heart Association awards 09PRE2060385 (JKM) and 10GRNT3490001 (RJP) and NIH grants T32-GM007267 (JKM), T32-HL007284 (JKM), R21-HL098632 (RJP), and R01-HL074082(RJP).

Disclosures: The authors have no conflicts to report. 


\section{Chapter 2 Figures}
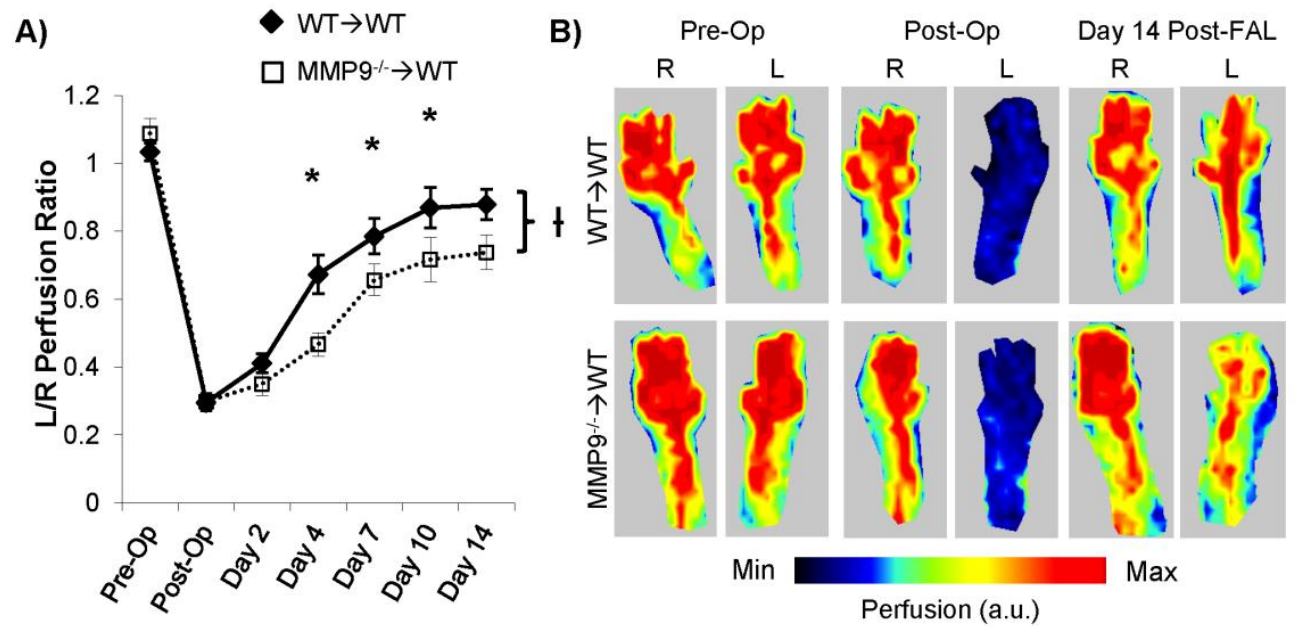

Figure 2.1

BMC-specific deletion of MMP9 impairs perfusion recovery after FAL. A) Laser Doppler perfusion recovery curve between WT $\rightarrow \mathrm{WT}$ and $\mathrm{MMPg}^{-/} \rightarrow \mathrm{WT}$ out to 14 days post-FAL ( $\mathrm{n}=8$ and 7 , respectively). B) Representative laser Doppler perfusion images in foot pads. * indicates $p<0.05$ between WT $\rightarrow W T$ and MMPg $^{-/} \rightarrow$ WT within the given time point. $f$ indicates $p<0.05$ between WT $\rightarrow$ WT and MMPg $\rightarrow$ WT recovery curves across all time points. 

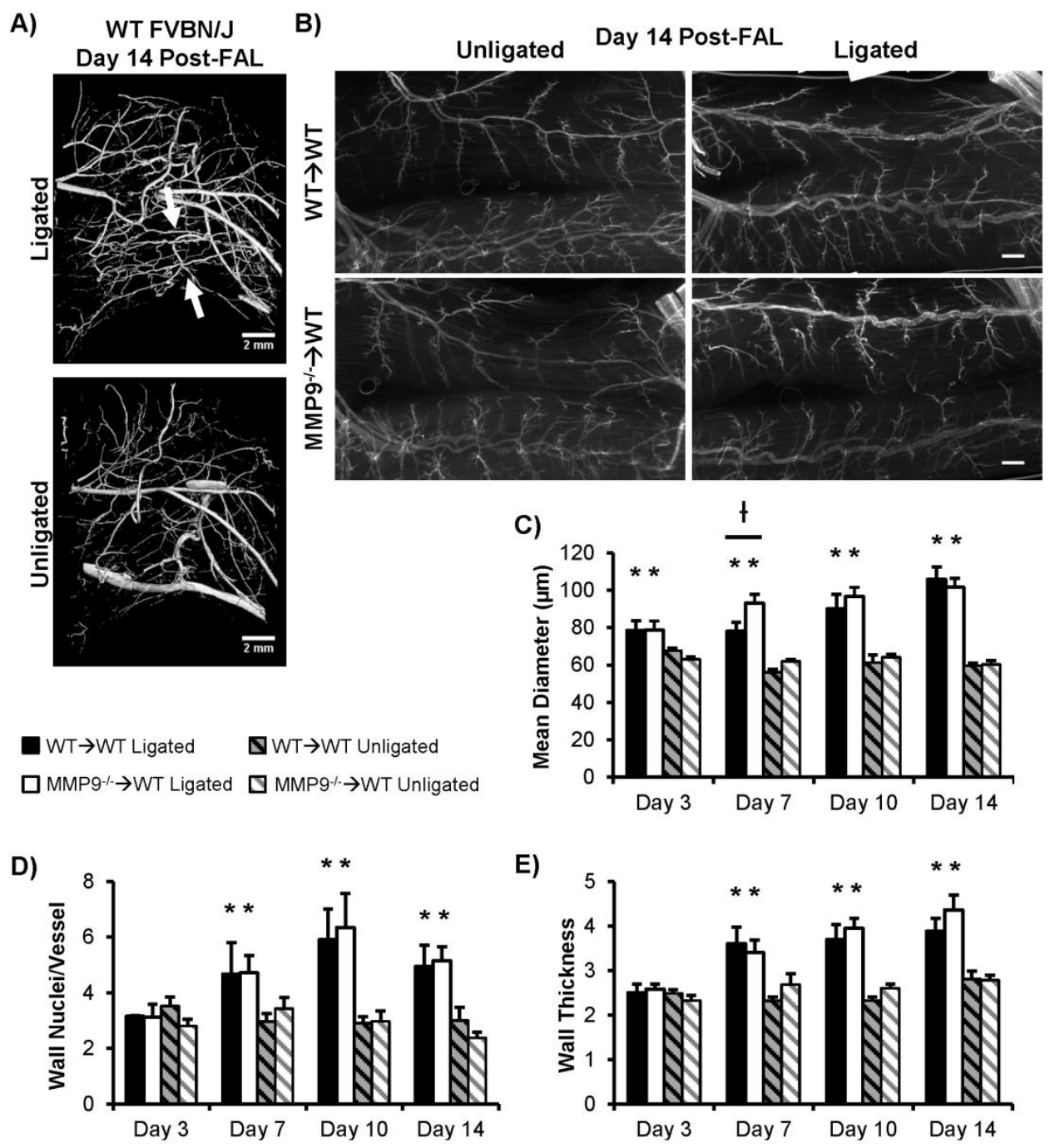

\section{Figure 2.2}

Arteriogenesis in gracilis adductor muscle after FAL. A) microCT images of mouse upper hindlimbs at 14 days post-FAL (age- and strain-matched WT mice). Arrows indicate primary collateral arteries in the gracilis adductor muscle. B) Representative gracilis muscle whole mounts of fluorescently labeled smooth muscle a-actin+ (SMaA) collateral arteries. Scale bars are $500 \mu \mathrm{m}$. C) Whole mount collateral artery diameters were quantified, showing outward remodeling of collateral arteries within the ligated limb starting at day 3 post-FAL. There were minimal differences between WT $\rightarrow$ WT and MMP9 ${ }^{-1-} \rightarrow W T$ mice ( $n=5-8$ per group). D,E) Cross-sections of collateral arteries stained with H\&E from the mid-zone of the gracilis muscle were quantified for wall nuclei and thickness, indicating significant hyperplasia and hypertrophy of the collateral artery wall within the ligated limbs of both WT $\rightarrow$ WT and MMP ${ }^{-1} \rightarrow$ WT mice starting at day 7 ( $n=5-8$ per group). * indicates $p<0.05$ between ligated versus unligated limbs within $\mathrm{WT} \rightarrow \mathrm{WT}$ or $\mathrm{MMPg}^{-/} \rightarrow \mathrm{WT}$ mice. + indicates $\mathrm{p}<0.05$ between WT $\rightarrow \mathrm{WT}$ versus $\mathrm{MMPg}^{-/} \rightarrow \mathrm{WT}$ mice within ligated or unligated limbs. 
A)

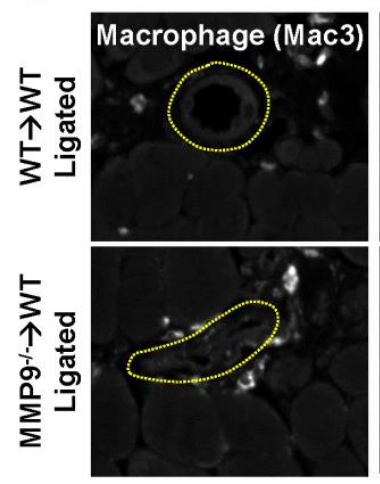

B)
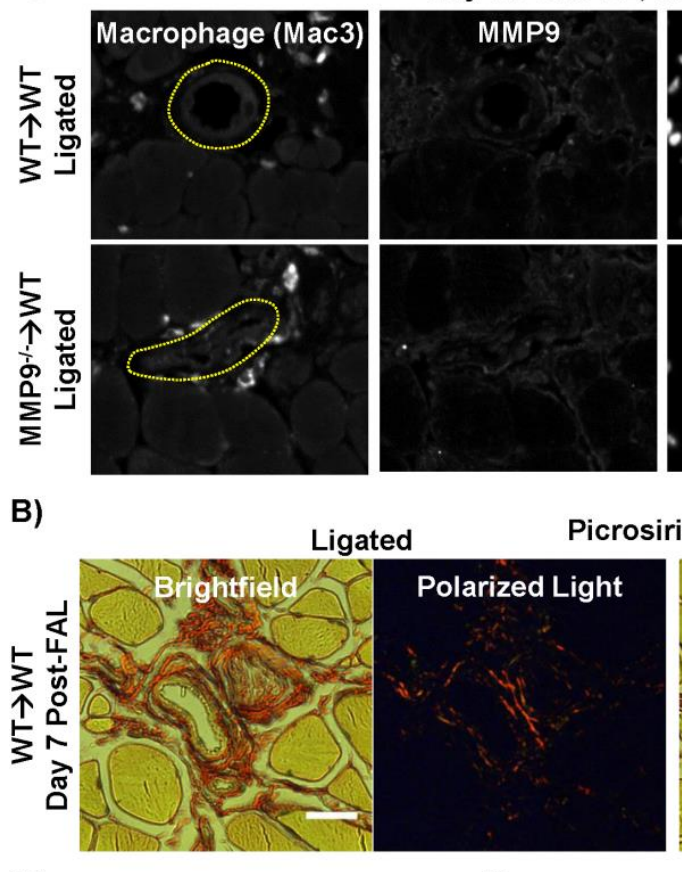

Day 3 Post-FAL, Gracilis Adductor
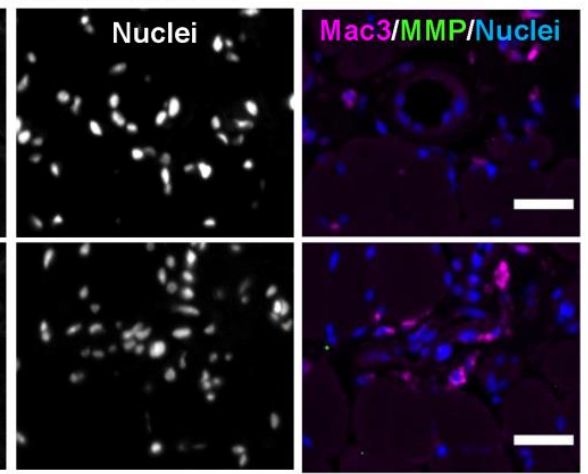

Unligated
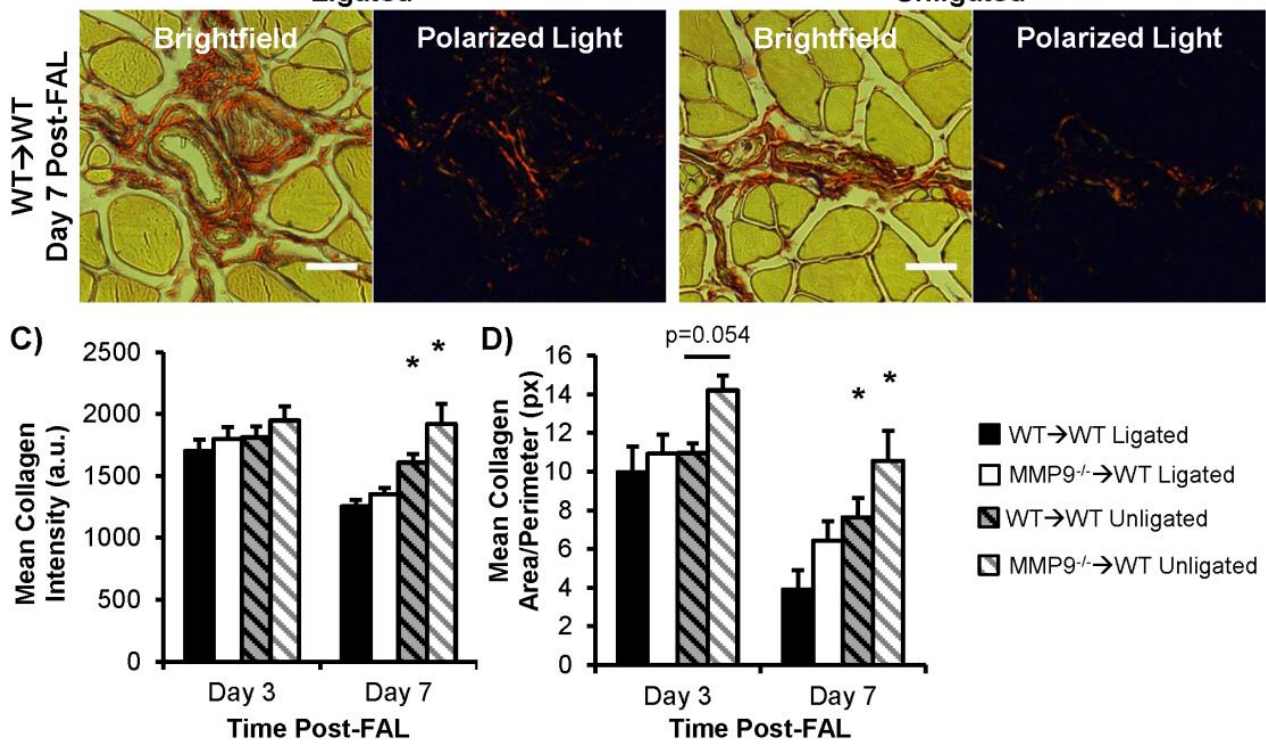

WT $\rightarrow$ WT Ligated

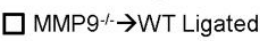

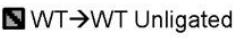

$\checkmark \mathrm{MMPg}-\mathrm{H}$ WT Unligated

\section{Figure 2.3}

BMC-derived MMP9 is not involved in collateral artery matrix remodeling. A) Immunofluorescence labeling of MMP9 in collateral artery cross-sections in the mid-zone of the gracilis muscle at 3 days postFAL showed minimal presence of MMP9 around collateral arteries or within Mac+ macrophages in either WT $\rightarrow$ WT or $\mathrm{MMPg}^{-1} \rightarrow \mathrm{WT}$ mice (see Figure 2.S1 for positive control). B) Polarized light birefringence of picosirious red stained collagen was used to quantify mean peri-collateral collagen intensity $(\mathrm{C})$ and area (D). All scale bars are $25 \mu \mathrm{m}$. * indicates $p<0.05$ between ligated versus unligated limbs within WT $\rightarrow$ WT or $\mathrm{MMPg}^{-/} \rightarrow \mathrm{WT}$ mice ( $\mathrm{n}=6-7$ per group). There were no significant differences between WT $\rightarrow \mathrm{WT}$ versus $\mathrm{MMPg}^{-/} \rightarrow$ WT mice within ligated or unligated limbs on either day 3 or day 7 . 


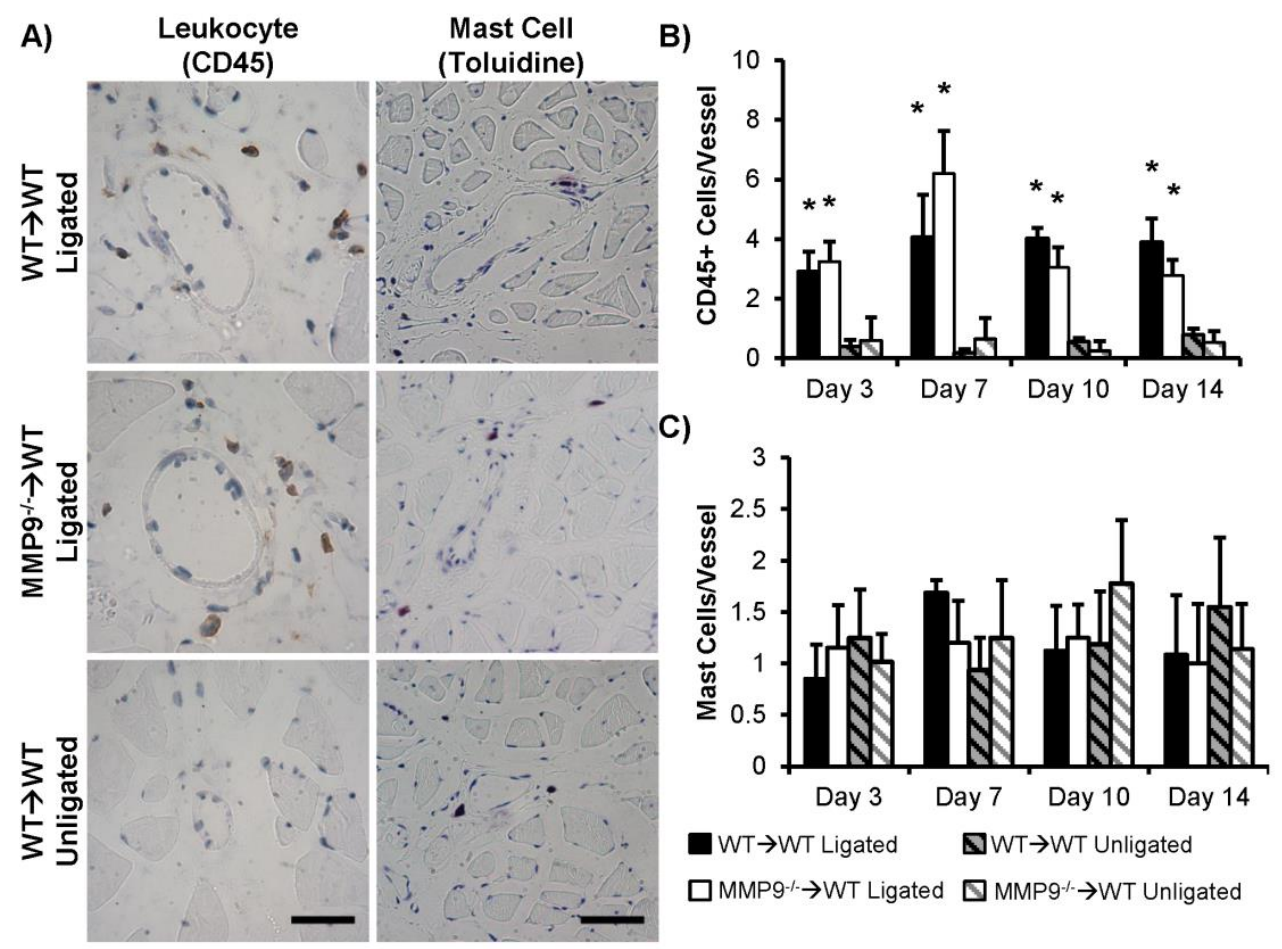

Figure 2.4

Recruitment of leukocytes and mast cells to remodeling collateral arteries in the gracilis muscle is unaltered in $\mathrm{MMPg}^{-1} \rightarrow \mathrm{WT}$ mice. A) Representative images of peri-collateral CD45-positive leukocytes (day 7 post-FAL) and toluidine blue positive mast cells (day 14 post-FAL) within gracilis collateral cross sections from ligated and unligated limbs. Scale bars are $25 \mu \mathrm{m}$. B, C) The mean number of CD45+ (C) or toluidine blue-positive (D) staining cells within $\sim 25$ microns around the artery wall shows leukocyte, but not mast cell, recruitment to the remodeling collaterals starting at day 3 post-FAL. ${ }^{*}$, indicates $p<0.05$ between ligated versus unligated limbs within WT $\rightarrow \mathrm{WT}$ or $\mathrm{MMPg}^{-/} \rightarrow \mathrm{WT}$ mice $(\mathrm{n}=5-8$ per group). There were no significant differences between WT $\rightarrow$ WT versus $\mathrm{MMP}^{-/-} \rightarrow \mathrm{WT}$ mice within ligated or unligated limbs at any time point. 

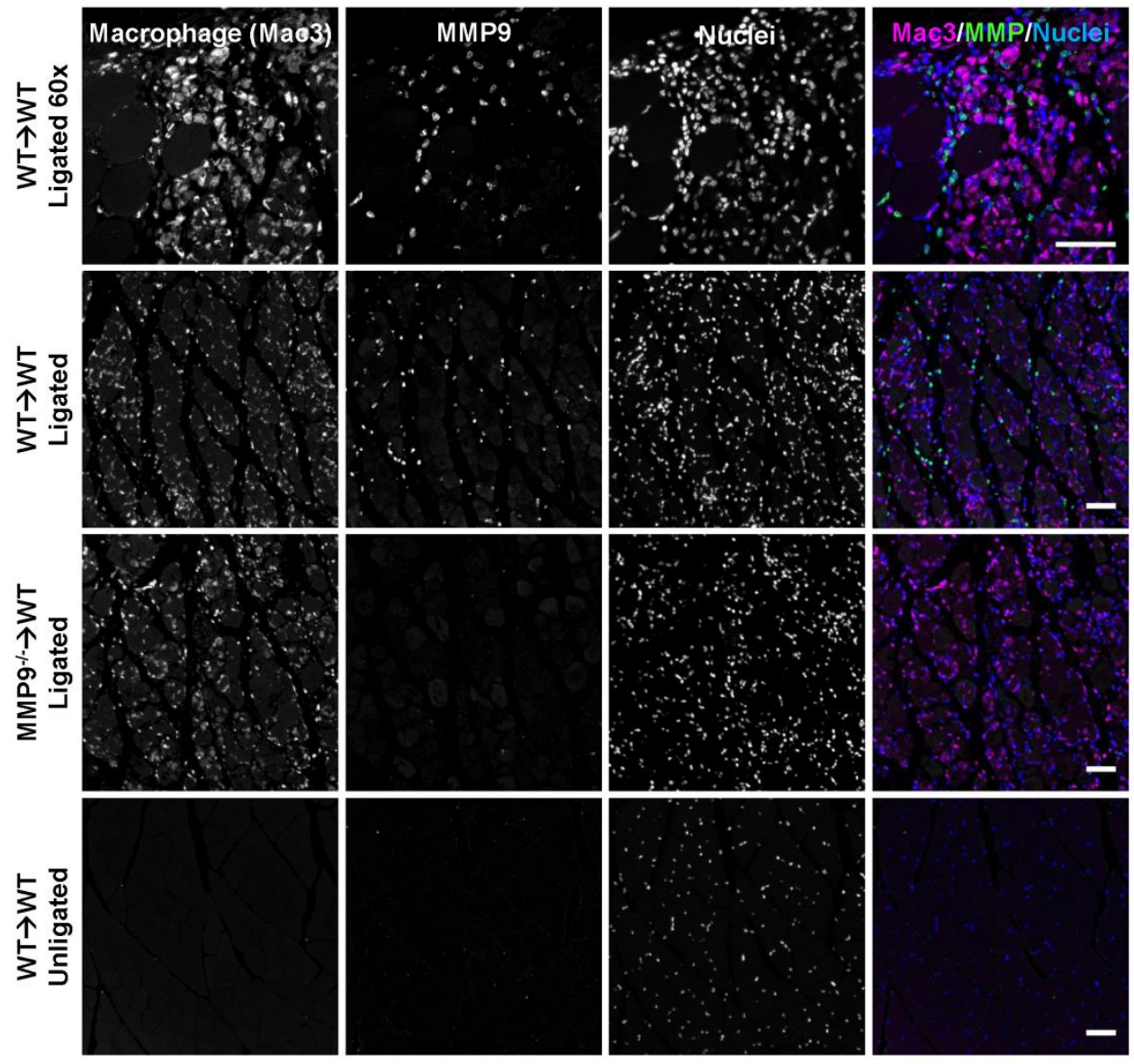

\section{Figure 2.5}

MMP9 is produced in BMCs recruited to the distal, ischemic muscle. Representative images from the ischemic calf muscle (oxidative region) at day 3 post-FAL show similar recruitment of Mac3+ macrophages to the ischemic tissue in both WT $\rightarrow$ WT and MMP9 $\rightarrow$ WT mice. Only WT $\rightarrow$ WT mice demonstrate MMP9+ cell staining, indicating these cells are of BMC origin. (Top row) High resolution imaging indicates these MMP9+ cells are not Mac3+ macrophages in WT $\rightarrow$ WT ischemic muscle. (Bottom row) Neither Mac3+ macrophages nor MMP9+ BMCs are present in unligated WT $\rightarrow$ WT calf muscle. All scale bars are $50 \mu \mathrm{m}$. 
A)
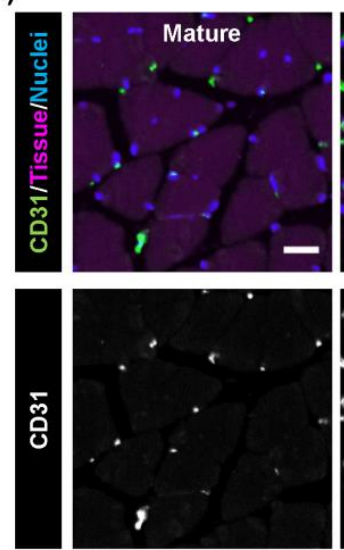

Day 14 Post-FAL
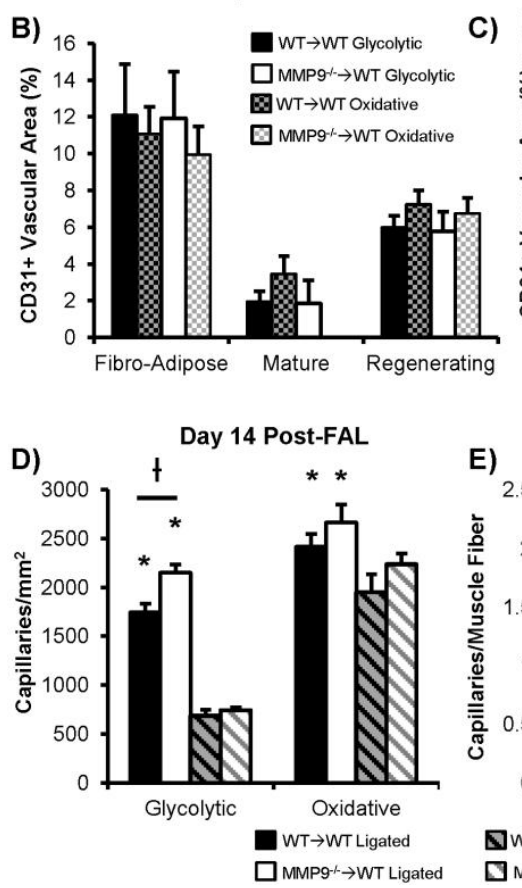

Ischemic Glycolytic Muscle, Day 14 Post-FAL
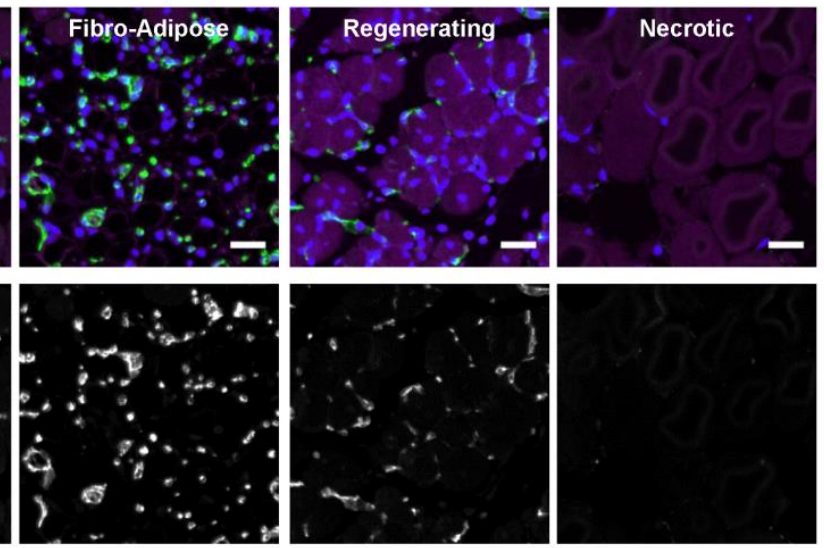

Day 14 Post-FAL
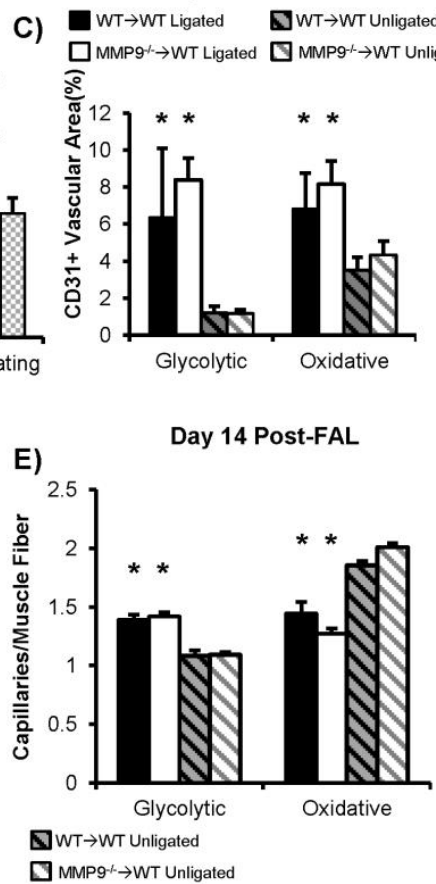

Day 3 Post-FAL

F)

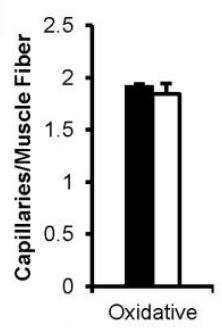

G)

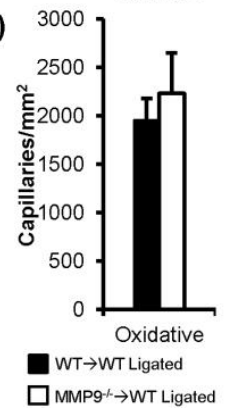

Figure 2.6

Capillary growth dynamics within ischemic calf muscle is unaltered in MMP9 $\rightarrow$ WT mice. A) Representative images of each of the 4 categories of classification for ischemic muscle based on tissue morphology (glycolytic region, 14 days post-FAL). Endothelial cells were fluorescently labeled with CD31 (green). Tissue auto-fluorescence (red) and nuclear counterstaining (blue) were used to define tissue structure. Scale bars are $25 \mu \mathrm{m}$. B,C) CD31+ vascular area was quantified for both the glycolytic (superficial) and oxidative (deep) regions of the calf muscle according to tissue type (B) or averaged across all tissue types (C) ( $n=7-8$ mice per group). D, E) Separately, CD31+ capillaries were quantified within regions of viable muscle (regenerating or mature fibers) on a per fiber basis for measures of capillary density (D) or capillary to muscle fiber ratio (E) ( $n=7-8$ mice per group). $F, G$ ) Capillaries within mixed (viable and necrotic) fiber regions at day 3 post-FAL were similarly quantified for capillary density $(F)$ and capillary to muscle fiber ratio $(G)(n=4$ mice per group). * indicates $p<0.05$ between ligated versus unligated limbs within WT $\rightarrow$ WT or MMPg $^{-1} \rightarrow$ WT mice. + indicates $p<0.05$ between WT $\rightarrow$ WT versus $\mathrm{MMPG}^{-/} \rightarrow$ WT mice within ligated or unligated limbs. 
A)
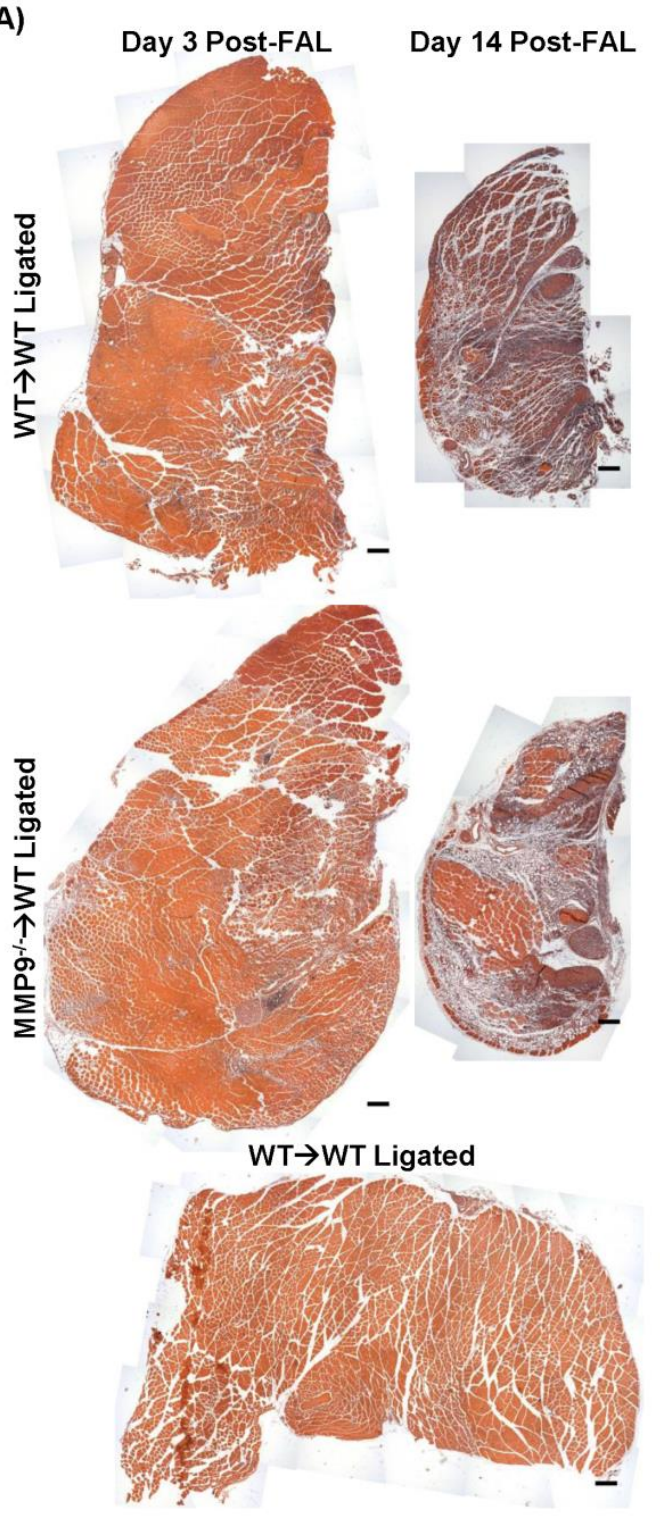

B) WT $\rightarrow$ WT Ligated

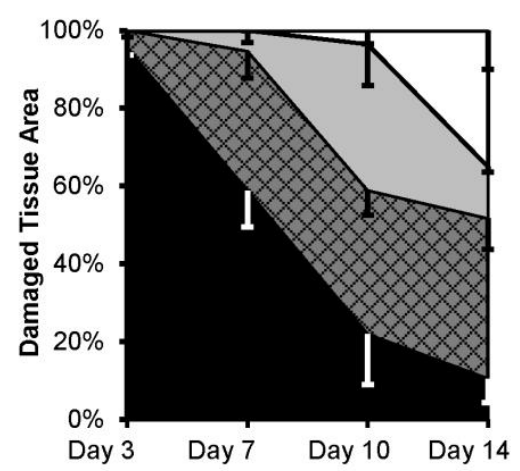

C) $\quad$ MMP9 $-1 \rightarrow$ WT Ligated

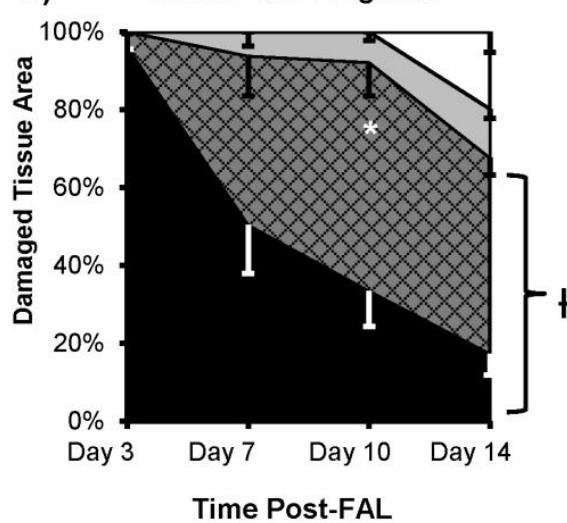

Necrotic Fiber $\square$ Early Regenerating

O Fibro-Adipose $\square$ Late Regenerating

\section{Figure 2.7}

$\mathrm{MMPg}^{-/} \rightarrow$ WT mice exhibit impaired skeletal muscle repair within damaged regions. A) Whole H\&E stained calf muscle cross-sections were analyzed by type tissue type (necrotic fibers, fibro-adipose tissue, early regenerating fibers, late regenerating fibers, and mature fibers). At 3 days post-FAL, tissue demonstrated large regions of frank necrosis (lack of muscle nuclei, and weakly eosinophilic cytoplasm). At day 14 post-FAL, muscles had significantly atrophied and undergone varying degrees of repair. Scale bars are $200 \mu \mathrm{m}$. B, C) Quantification of tissue composition within damaged tissue regions demonstrated that $\mathrm{MMPg}^{-/} \rightarrow \mathrm{WT}(\mathrm{B})$ had decreased muscle fiber regeneration compared to WT $\rightarrow \mathrm{WT}$ (C) mice. * indicates $\mathrm{p}<0.05$ for the percentage of regenerating (late and early stage) fiber area within damaged tissue between WT $\rightarrow$ WT versus MMPg $^{-/} \rightarrow W T$ mice at the given time point ( $n=5-8$ per group). $t$ indicates $\mathrm{p}<0.05$ between $\mathrm{WT} \rightarrow \mathrm{WT}$ versus $\mathrm{MMPg}^{-/-} \rightarrow \mathrm{WT}$ mice across all time points. 

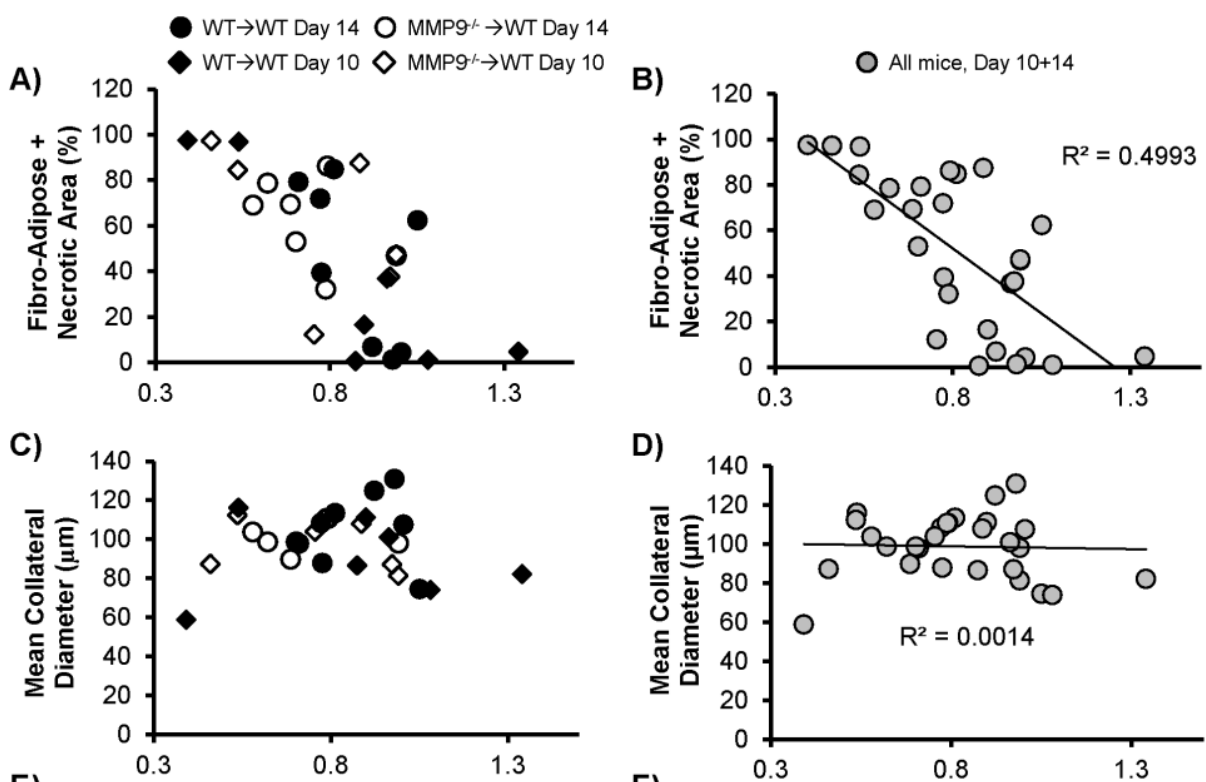

E)
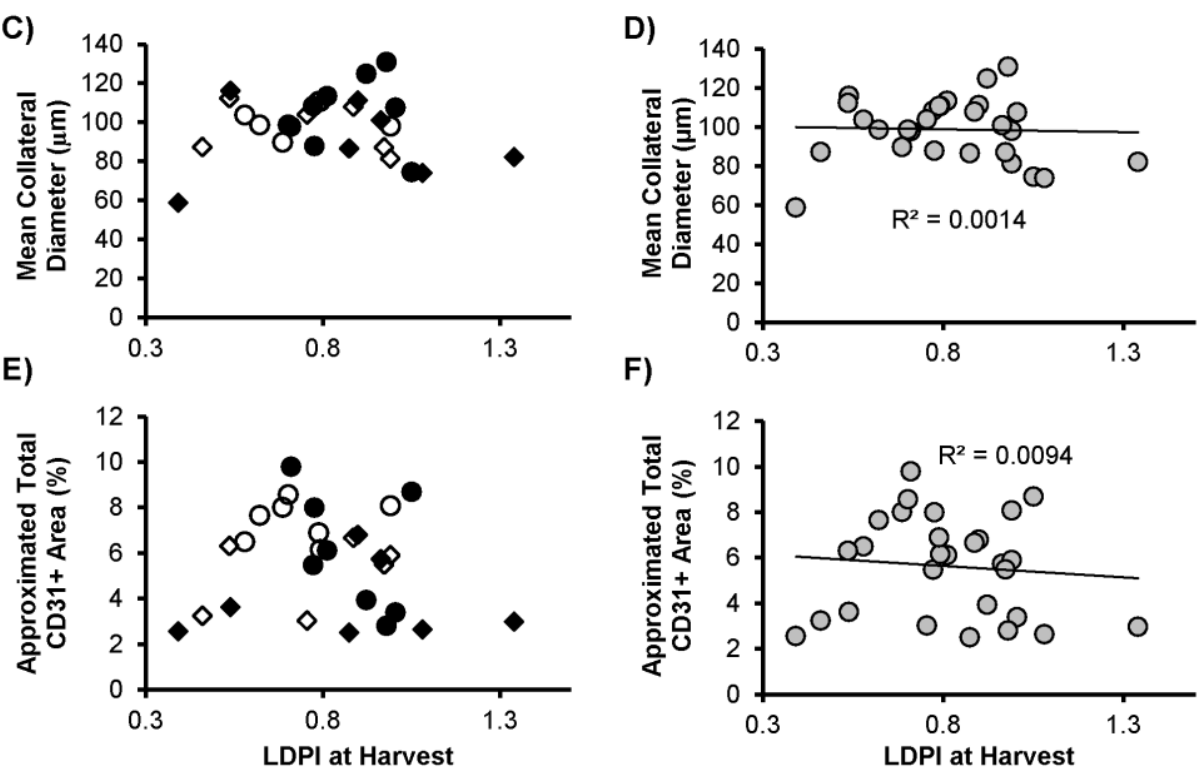

\section{Figure 2.8}

Perfusion is related to skeletal muscle repair, but not vascular remodeling. A, C, E) Laser Doppler L/R foot perfusion (LDPI) ratios at the day of harvest were compared to percent total fibro-adipose and necrotic tissue area per calf muscle cross sections, mean gracilis collateral diameter, or approximate CD31+ vascular area per calf muscle cross section for mice harvested at 10 and 14 days post-FAL. B, D, F) Values for all mice were then analyzed by linear regression (line). $R^{2}$ coefficient is displayed for each regression. Only the percentage fibro-adipose and necrotic tissue area showed significant correlation with LDPI perfusion ratio at harvest $(p<0.001)$. 

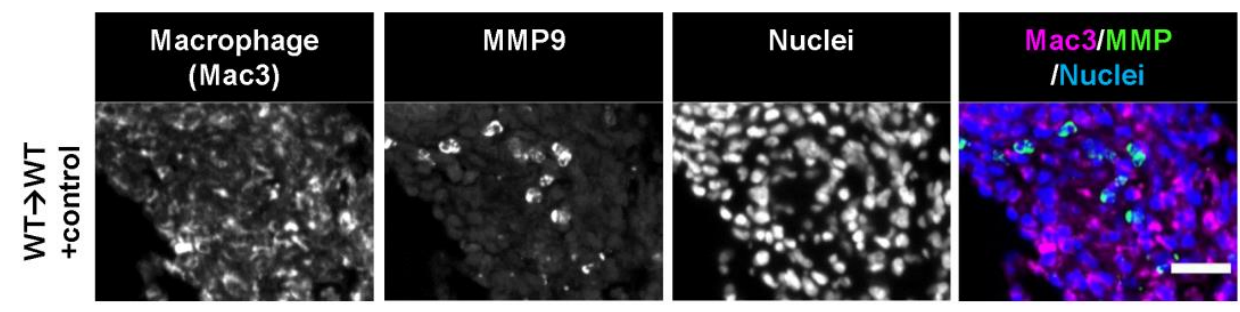

\section{Figure 2.S1}

Positive control of Mac3 and MMP9 staining for day 3 post-FAL gracilis cross sections. Granulomatous tissue within WT $\rightarrow$ WT mouse tissue was identified nearby gracilis adductor muscle. This positive control demonstrates clear MMP9 (green) labeling in Mac3-negative inflammatory cells (magenta). Scale bar is $25 \mu \mathrm{m}$. 


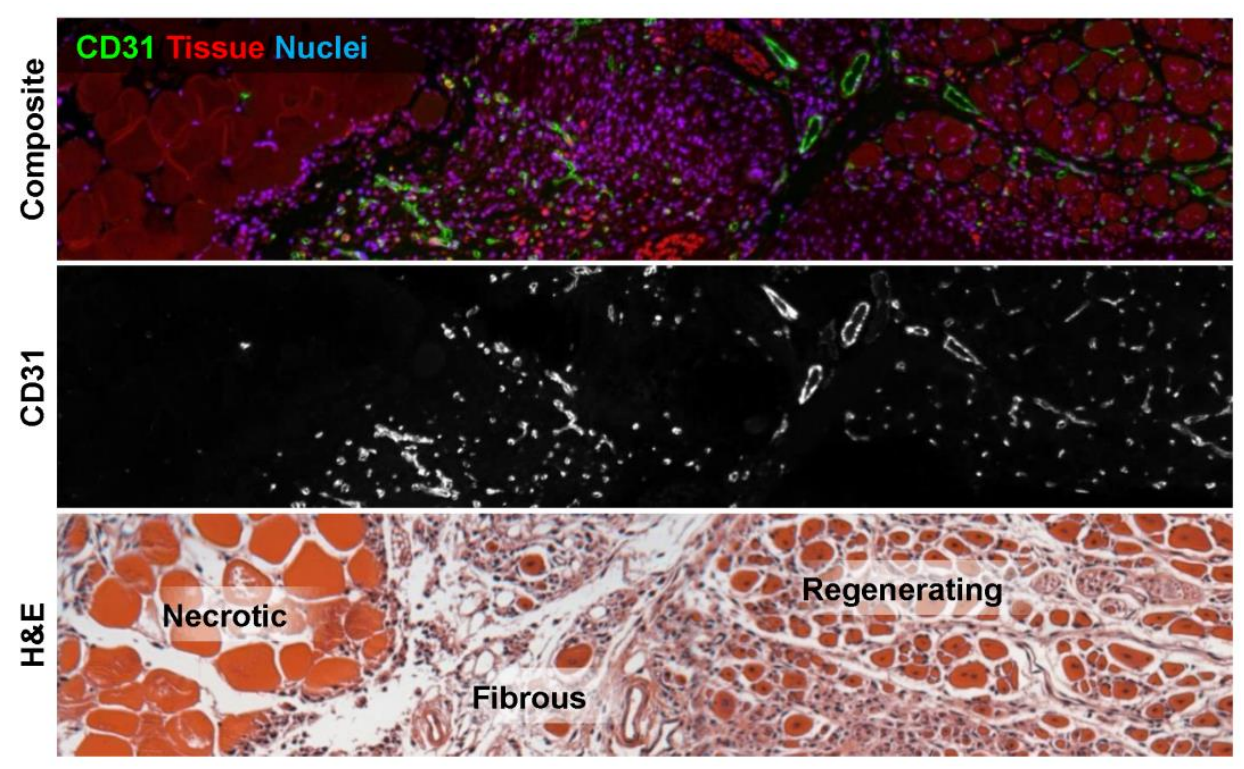

\section{Figure 2.S2}

Ischemic gastrocnemius muscle showed heterogeneity of muscle regeneration and damage. (Top, middle) Ischemic WT $\rightarrow$ WT gastrocnemius tissue from 14 days post-FAL showed spatial variation in CD31+ immunofluorescence labeling. (Bottom) Corresponding tissue structure can be seen from a serial section stained with H\&E. Regions that would be classified as necrotic, fibrous, or regenerating are denoted. 
Chapter 3: Myoglobin Overexpression Inhibits Reperfusion in the Ischemic Mouse Hindlimb Through Impaired Angiogenesis but Not Arteriogenesis.

Joshua K. Meisner, Ji Song, Brian H. Annex, Richard J. Price

(in preparation) 


\section{Introduction}

Peripheral arterial disease (PAD) is a chronic disease caused by the progressive and acute occlusion of the peripheral vasculature at atherosclerotic lesions. Primarily occurring in the lower limbs, progressive PAD ultimately leads to the debilitating consequences of intermittent claudication and critical limb ischemia. With high prevalence $(>20 \%$ of those $>65$ years of age (8)) and economic impact ( $\$ 4.4$ billion estimated treatment costs (9)) of PAD but limited therapeutic options, there is a critical need for developing new therapeutic modalities. One promising approach is therapeutically stimulating adaptive vascular remodeling to slow and, potentially, reverse the course of PAD. To date, however, trials using this approach have largely failed. An improper understanding of the balance of angiogenesis versus arteriogenesis has been cited as a primary reason for many of these failures $(4,6,7)$.

The adaptive vascular remodeling process can be broken down into two aspects. First, in ischemic tissues downstream of an arterial occlusion, capillaries grow from existing vessels via angiogenesis, expanding blood flow distribution throughout the ischemic tissue. In contrast, collateral arteries around the occlusion are stimulated to undergo structural lumenal expansion (i.e. arteriogenesis), which allows for greater in-flow into the distal, ischemic tissue. However, therapeutic trials have largely focused their therapeutic effect predominantly toward only one process (angiogenesis or arteriogenesis), but not both $(4,6,7)$. The most direct example of an unbalanced approaches comes from the early and prominent failures of a number of large clinical trials using predominantly angiogenic factors (e.g. VEGF and HIF1alpha) to induce angiogenesis as a means of enhancing revascularization (177-179). However, clinical trials targeting factors chosen specifically for their arteriogenic potential (e.g. FGF2, GM-CSF) have also reported only marginal success (17,180-182). A more fruitful strategy was recently hinted at in a study by West et al (183), which suggested that even with increase perfusion pressure to the distal tissue after a percutaneous intervention, revascularization is unable to restore 
microvascular perfusion in PAD patients. This suggests that even with functional arteriogenesis and subsequent restoration of normal perfusion pressure downstream of an occlusion, there are still microvascular perfusion impairments in PAD that must be addressed for full functional recovery. In sum, these findings suggest the need to better understand how angiogenesis and arteriogenesis work together to improve reperfusion after arterial occlusion.

Novel data demonstrating how angiogenesis and arteriogenesis separately contribute to reperfusion after arterial occlusion could significantly help bridge the divide in the field by outlining how targeting both aspects of neovascularization could optimize the potential for therapeutic success. However, being able to identify how angiogenesis and arteriogenesis each contributes to the reperfusion response to ischemic injury requires both a condition which does not simultaneously impact arteriogenesis and angiogenesis and methods that can separately quantify angiogenesis and arteriogenesis. Myoglobin-over expression in skeletal muscle $\left(\mathrm{MbTg}^{+}\right)$may serve as a unique stimulus that has an effect on tissue reperfusion during ischemia but uncoupled effects on arteriogenesis and angiogenesis. $\mathrm{MbTg}^{+}$has previously been documented to impair angiogenesis and reperfusion in a severe hindlimb ischemia model (184). The impaired angiogenesis was proposed to arise from the excess myoglobin acting as a sink for nitric oxide $(184,185)$, which results in the loss of the key trophic and angiogenesis factor during ischemia $(122,186)$. The tight association of the endothelium with muscle fibers required for this mechanism occurs at the capillary level, but the greater separation of collateral arteries from the parenchymal muscle tissue reduces the potential for this mechanism on arteriogenesis $(187,188)$. The potential angiogenesis-specific impairment is further supported by the delayed time-course of the perfusion deficit $(151,184)$. However, because of the more severe ischemia model chosen, the ability to assess arteriogenesis was limited (155). Alternatively, a milder hindlimb ischemia, femoral artery ligation ( $F A L)$, model can be used to produce consistent collateral artery remodeling needed for the precise quantification of arteriogenesis 
$(142,154,155)$. Therefore, we hypothesize that coupling the $\mathrm{MbTg}^{+}$transgenic model with the milder FAL ischemic stimulus will yield the first dataset to quantify the degree to which impairment in angiogenesis alone can contribute to the reperfusion response after arterial occlusion. These data would present a unique, and presently lacking, demonstration that angiogenesis is required along with normal arteriogenesis for a more fully effective revascularization response.

\section{Material and Methods}

\section{Animals}

All animal protocols were approved by the Institutional Animal Care and Use Committee at the University of Virginia and conformed to all regulations for the ethical use of animals as outlined in the American Heart Association Guidelines for the Use of Animals in Research. The intact myoglobin gene and promoter regions of transgenic mice $\left(\mathrm{MbTg}^{+}\right)$produces a moderate level ( $200 \%$ of normal) of myoglobin over-expression specific to cardiac and skeletal muscle tissue, matching endogenous myoglobin expression $(184,189)$. Transgenic mice were backcrossed for nine generations into $\mathrm{C} 57 \mathrm{BI} / 6$ mice before use. $\mathrm{MbTg}^{+}$and $\mathrm{WT}$ littermates were identified using previously described markers $(184,189)$. Only male mice were used for this study.

\section{Femoral Arterial Ligation Model}

Femoral arterial ligation (FAL) was performed similar to that previously described $(99,112,154,155)$. The protocol produces consistent remodeling of the collateral arterial pathway traversing the gracilis adductor muscle, representative of upper hindlimb arteriogenesis, and produces a moderate level of ischemia in the downstream tissue. Age-matched mice (5.3 \pm 0.9 (standard deviation) months of age) were anesthetized (i.p $120 \mathrm{mg} / \mathrm{kg}$ ketamine, $12 \mathrm{mg} / \mathrm{kg}$ xylazine, and $0.08 \mathrm{mg} / \mathrm{kg}$ atropine) and prepared for aseptic surgery. On the left leg, a small 
incision was made to expose the femoral artery, which was then isolated from the femoral vein and nerve. Two ligatures of 6.0 silk sutures were placed around the femoral artery; one immediately distal to the epigastric artery with the second proximal to the saphenous-popliteal artery bifurcation. The artery segment between the two ligatures was then severed, and the surgical site was closed. A second, sham operation was performed on the opposite (i.e. right) limb, wherein the femoral artery was exposed but not ligated. Animals received injections of bupernorphine for analgesia immediately post-procedure and 8-12 hours later.

\section{Functional Assessment of Ischemic Injury}

To quantify the extent of functional injury induced by FAL, at various time points mice were assess for post-operative weight loss/gain and for foot use. Foot use score was assessed similar to that previously described (112), with each scored defined as: 0 , normal use; 1 , no flexion of the toes; 2, no plantar flexion; 3 , dragging of the foot.

\section{Laser Doppler Perfusion Imaging}

Laser Doppler perfusion imaging was performed to monitor blood flow recovery in response to FAL as previously described $(99,154)$. Briefly, mice were anesthetized (i.p 120 $\mathrm{mg} / \mathrm{kg}$ ketamine, $12 \mathrm{mg} / \mathrm{kg}$ xylazine, and $0.08 \mathrm{mg} / \mathrm{kg}$ atropine) and placed prone on a surgical heating pad for 5 minutes to minimize temperature variation and allow imaging of the soles of the feet. The lower limbs were scanned (Lisca PIM laser Doppler imager), and mean voltage of foot perfusion was used to calculate relative perfusion ratio (ligated/unligated).

\section{Tissue Harvesting}

To minimize differences in collateral arterial tone across mice, immediately prior to tissue collection, gracilis muscles of anesthetized mice were exposed and drip perfused with $10^{-4} \mathrm{M}$ adenosine in warmed Ringer's physiologic saline solution. Mice were then euthanized by an 
overdose of anesthetic and then flushed with $2 \%$ heparinized saline and perfusion fixed with $10 \%$ neutral buffered formalin via cardiac cannulation. After 30 minutes of fixation, gracilis muscles were removed for whole mount immunofluorescence analysis, and calf muscles (containing the gastrocnemius and plantaris muscles) were removed and prepared for paraffin embedding by the University of Virginia Research Histology Core.

\section{Whole Mount Immunofluorescence and Collateral Network Image Analysis}

Harvested gracilis muscles were labeled by immunofluorescence for smooth muscle $\alpha$ actin to visualize the collateral vasculature, similar to that previously described $(99,154)$. Immediately after harvesting, gracilis muscles were washed, and then incubated with 1:200 antismooth muscle a-actin - Cy3 (1A4 clone, Sigma Aldrich, St Louis, MO) antibody in blocking buffer (PBS with $0.1 \%$ saponin, $2 \%$ BSA, and $0.2 \%$ sodium azide) for 72 hours at $4^{\circ} \mathrm{C}$. Gracilis muscles were then washed and cleared in $50 \%$ glycerol in PBS overnight at $4^{\circ} \mathrm{C}$. Clear tissues were mounted between two coverslips using $500 \mu \mathrm{m}$ thick spacers (645501, Grace Bio-Labs) to keep constant thickness between muscles. Muscles were imaged at $4 \mathrm{x}$ magnification on a Nikon TE300 wide-field, fluorescence microscope with a CCD camera (Microfire, Optronics, Goleta, CA). Individual fields of view were montaged together for network analysis (Photoshop CS2, Adobe Systems Inc).

Each head of the gracilis muscle (anterior and posterior) contained one primary collateral pathway that connected the muscular branch artery (also known as the profundus femoris or lateral caudal femoral artery) to the saphenous artery (see Figure 3.2). Diameter was measured at 9 evenly spaced intervals along the length of the each primary collateral using the Fiji image analysis platform (http://fiji.sc) (156). The diameter measurements across both heads of the gracilis muscles were pooled to determine mean collateral diameter per muscle. 


\section{Cross Section Immunofluorescence}

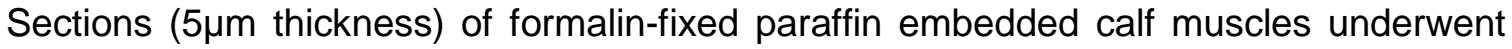
immunofluorescence labeling for CD31 (PECAM1) analysis of capillary density. Dewaxed and rehydrated slides underwent heat mediated antigen retrieval in a 1200W microwave for 20 minutes in citrate-based antigen retrieval buffer (Vector Laboratories, H-3300). Slides were then blocked and labeled with primary antibody [rat anti-CD31 (1:100, SZ31 clone, HistoBioTec LLC, Miami Beach, FL)] overnight at $4^{\circ} \mathrm{C}$. Slides were then washed and incubated with goat-anti rat Alexa Fluor 647 (1:200, Life Technologies) for 45 minutes at room temperature. Nuclei were counterstained using 10nM SYTOX green (Life Technologies). Slides were then sealed with Prolong Gold (Life Technologies) to minimize photo-bleaching.

\section{Capillary Growth (Angiogenesis) Analysis}

Cross sections of calf muscle stained for CD31 were used to determine capillary density metrics. Because there are at two largely distinctive regions of the harvested calf muscle with significantly different capillary and muscle fiber composition, analysis was separated into two regions: the superficial region, comprised of the white gastrocnemius muscle, and the deep region, comprised of the plantaris and red gastrocnemius muscle, termed here as the glycolytic and oxidative regions, respectively (157-159). Two fields of view from each region in each section (two separate sections per muscle) were imaged at 20x magnification on a Nikon TE2000 C1 laser scanning confocal microscope. Number of capillaries (CD31+ structures $<7 \mu \mathrm{m}$ in diameter), mature and regenerating muscle fibers (identified from auto-fluorescence), and muscle area were counted in each field of view using Fiji image analysis software. The presence of centrally located nuclei was used to identify regenerating fibers. Each field of view yielded >100 and >200 muscle fibers per glycolytic or oxidative region per muscle, respectively. 


\section{Cross Sectional Damage Analysis of Ischemic Tissue}

For measurements of skeletal muscle damage, sections $(5 \mu \mathrm{m}$ thickness) of paraffin embedded calf muscle were cut and stained with H\&E at the University of Virginia Research Histology Core. A high resolution image of the entire muscle cross section was reconstructed by stitching together individual fields of view taken at 10x magnification on a Nikon TE300 microscope with color CCD camera (Microfire, Optronics Inc). In the event of partial cross section, the section was excluded from analysis. At least one of two sections was usable in all harvested muscles. Tissue composition was classified into five categories defined as followed: 1) necrotic, where fibers lacked nuclei, were rounded and dilated in appearance, and had weak eosiniphilic cytoplasm; 2) early regenerating, where fibers were small, loosely organized, and had centrally located nuclei; 3 ) late regenerating, where fibers were approximately the size of mature fibers within the given region, compactly organized, but still maintained centrally located nuclei; 4) fibro-adipose tissue, where there was minimal presence of myoblasts and was dominated by fibrous matrix and adipose tissue; 5) mature fibers, where muscle fibers were comparable in size, organization, and structure for the respective region in unligated control limb. Individual regions of each category were manually identified and measured for calculating percent area.

\section{Statistics}

All results are reported as \pm standard error of the mean. All images were randomized and de-identified to enable blinded analysis. All data were first tested for normality. Statistical significance was assessed by one- and two-way ANOVA, followed by paired comparisons using the Holm-Sidak method for multiple comparisons (SigmaStat 3.5, Systat Inc). Significance was assessed at $\mathrm{P}<0.05$. 


\section{Results}

\section{Myoglobin Over-expression Impairs Perfusion and Functional Recovery after FAL}

The time course of reperfusion after FAL was assessed by Laser Doppler Perfusion Imaging (LDPI) in myoglobin over-expression mice $\left(\mathrm{MbTg}^{+}\right)$and control littermates (WT) (Figure 3.1). Both $\mathrm{MbTg}^{+}$and WT mice show a perfusion deficit only within the first 7 days post-FAL, with a return to baseline perfusion levels by 10 days post-FAL (Figure 3.1A). After day 10, perfusion values showed higher perfusion in the ligated limb during ketamine and xyaline induced anesthesia. Within the window of reduced perfusion during first 7 days post-FAL, there is a significant deficit in perfusion within the $\mathrm{MbTg}^{+}$mice (Figure 3.1A, 3.1B). This perfusion recovery deficit is further reflected in a functional deficit in terms of post-operative weight loss and foot use score within the $\mathrm{MbTg}^{+}$mice (Figure 3.1C, 3.1D). However, both foot use and weight were fully restored by day 28 post-FAL in both groups (no mice had foot use $>0$ in either group; $-1.89 \pm 0.38 \mathrm{~g}$ and $-1.36 \pm 0.31 \mathrm{~g}$ weight change for $\mathrm{MbTg}^{+}(\mathrm{n}=11)$ and WT $(n=7)$ mice, respectively, $p=0.33)$.

\section{Myoglobin Over-expression Does Not Alter Arteriogenesis in Response to FAL}

As previous demonstrated, the FAL method used produced consistent arteriogenesis in the collateral pathways with the gracilis adductor muscle (Figure 3.2) $(142,154,155)$. Immunofluorescence labeling of smooth muscle $\alpha$-actin was used for the identification and measurement of collateral artery diameter along the length of the collateral pathway spanning the gracilis muscle (Figure 3.2A). Both $\mathrm{MbTg}^{+}$and WT mice showed a similar degree of outward remodeling (i.e. arteriogenesis) within the ligated limb by 7 days post-FAL that was maintained out to day 28 post-FAL, without further expansion (Figure 3.2B). This matches the reperfusion time course, with limited additional gains in foot perfusion after 7 days post-FAL. At no time did the mean collateral diameters within the ligated or unligated limb significantly differ between 
$\mathrm{MbTg}^{+}$and WT groups, showing no indications of differences in baseline or remodeled collateral diameters (Figure 3.2B).

\section{Myoglobin Over-expression Delays Skeletal Muscle Recovery, but Does not Alter Capillary Density Once Ischemia is Resolved}

To determine if the impaired perfusion recovery seen in the $\mathrm{MbTg}^{+}$mice was related to impairments in capillary remodeling in the downstream ischemic tissue, capillary and muscle fiber structures were analyzed in cross sections of calf muscle. Because of the spatial clustering of muscle fiber type in the muscle $(159,190)$ and the variation in MbTg expression across fiber types and muscle groups (189), analysis was divided into glycolytic (superficial, white gastrocnemius muscle) and oxidative (deep, plantaris and red gastrocnemius muscle) regions. The glycolytic regions show predominance of type IIB and IIDB muscle fibers, while the oxidative regions of the muscle show a mix of types IID, IIAD, IIA, and I muscle fibers within the c57bl6 strain $(158,190)$. As expected from these variations in fiber type, glycolytic regions showed larger average fiber size (Figure 3.3E) and lower capillary to muscle fiber ratio (Figure 3.3C) than oxidative regions. Similar to previous results (184), there were no significant differences seen between WT and $\mathrm{MbTg}^{+}$mice at baseline in terms of capillary to muscle fiber ratio or muscle fiber size in either glycolytic or oxidative regions. Not surprisingly, by $>2$ weeks after returning to baseline perfusion, there were only moderate effects on capillary to muscle

fiber ratio in the ischemic limbs of either WT or $\mathrm{MbTg}^{+}$mice at 28 days post-FAL. Specifically, there was an increase in capillary to muscle fiber ratio and capillary density within the $\mathrm{MbTg}^{+}$ mice in the glycolytic regions (Figure 3.3C). This increase in capillarity occurred in a setting of delayed regeneration, where $\mathrm{MbTg}^{+}$mice showed a larger presence of regenerating fibers than WT mice, which showed few regenerating fibers by 28 days post-FAL (Figure 3.3F). Additionally, there was an increase in capillary density within the oxidative regions of the muscle (Figure 3.3D). Further, the increased presence of regenerating fibers within the glycolytic region 
of $\mathrm{MbTg}^{+}$mice translated into increased regenerating fibers across the entire calf muscle cross section (Figure 3.3G). These findings coincide with the decreased functional recovery of $\mathrm{MbTg}^{+}$ mice after ischemic ligation (Figure 3.1).

\section{Discussion}

\section{Summary}

Our results suggest that impairment in angiogenesis alone is sufficient to impair perfusion recovery after arterial occlusion. We base these conclusions on three key pieces of information from the response to hindlimb ischemia in $\mathrm{MbTg}^{+}$mice. 1) We confirmed the deficit in reperfusion capacity of $\mathrm{MbTg}^{+}$that was previously identified (Figure 3.1) (184). In the ischemia model chosen here, the more distal femoral arterial ligation procedure allowed for full perfusion recovery to baseline levels by 10 days after $\mathrm{FAL}$ (Figure 3.1), but $\mathrm{MbTg}^{+}$mice showed decreased reperfusion during this initial ischemic window. While this milder ischemic stimulus decreased the extent of functional tissue damage and duration of reperfusion deficit, it allowed for the quantification of potential differences in the arteriogenic capacity within the two strains (Figure 3.2). 2) When arteriogenic capacity was quantified, it demonstrated that the deficit in perfusion capacity seen in $\mathrm{MbTg}^{+}$mice could not be attributed to impaired arteriogenesis. 3) Previously it was shown $\mathrm{MbTg}^{+}$mice show impairments in their angiogenic capacity when some ischemia is still present in the distal muscle (184) at 28 days after a more severe ischemic stimulus. While, these differences were not present with $>2$ weeks after ischemia resolution here, the impact of impaired angiogenesis during the ischemic window of the ischemia model used here is likely to have attributed to the decreased perfusion recovery seen in $\mathrm{MbTg}^{+}$mice during the first 7 days post-FAL, when angiogenesis is more prominent (26). Investigation is ongoing to confirm these finding at the earlier day 7 post-FAL time point, where there is a significant deficit in perfusion capacity in $\mathrm{MbTg}^{+}$mice. Together the current data and the 
previous data provide evidence that microvascular impairment in capillary growth within the distal ischemic hindlimb is sufficient to impair limb function and reperfusion without any impairment in upstream arteriogenesis.

\section{Myoglobin Overexpression Preferentially Impacts Glycolytic Muscle Fibers}

Interestingly, the delayed regeneration seen in the $\mathrm{MbTg}^{+}$group suggests that overexpression of myoglobin appears to have a more pronounce effect within glycolytic muscle than in oxidative muscle. This pattern appears somewhat opposite from initial expectations. The promoters used for the transgene yield an over-expression directly proportional to endogenous expression (189); however, in the normal state, there is minimal myoglobin expression seen within the superficial (glycolytic) region of the gastrocnemius muscle $(189,191)$. This would suggest the effect of increasing myoglobin expression would have a more pronounced effect on the response to ischemia in the oxidative region versus the glycolytic region, if the mechanism of impaired angiogenesis is through acting as a sink for nitric oxide as previously proposed (184). However, hypoxia alters myoglobin expression (191-193), which would subsequently dictate over-expression in the $\mathrm{MbTg}^{+}$mice (189). These effects vary by muscle fiber type (191), which suggest differential response between glycolytic and oxidative regions. However, how these elements integrate over the course of ischemia and regeneration is poorly understood. Given myoglobin expression can be activated by hypoxia, it may be the increase in myoglobin from the minimal levels pre-ischemia within the glycolytic muscle constitutes a larger relative increase in myoglobin within the $\mathrm{MbTg}^{+}$mice, yielding a larger relative effect within the glycolytic regions of the ischemic muscle.

\section{Relative Contribution of Arteriogenesis and Angiogenesis}

Understanding the balance of how arteriogenesis and angiogenesis contribute to the vascular reperfusion response is critical for determining the optimal approach for therapeutic 
revascularization. Few studies have made detail analysis of the relative contribution of the collateral vasculature versus the remodeling of the distal microcirculation to the decrease resistance of the vascular tree downstream of an arterial occlusion. However, those that have clearly demonstrate that the arteriogenesis and the expansion of the pre-existing collateral circulation are the predominant contributors to lowering the resistance imposed by the occlusion of a major artery (see review (3)). A similar observation is present in the current data, whereby the collateral growth seen at 7 days post-FAL correlates well with the large increase in perfusion within the first several days. However, there is still a perfusion deficit within the $\mathrm{MbTg}^{+}$mice at the time, and perfusion still continues to increase despite no evidence of further arteriogenesis. While this evidence does not conflict with the well-founded conclusions that arteriogenesis is the most efficient means of lowering bulk resistance to the distal tissue $(3,26,142)$, it does not preclude angiogenesis from still playing a significant-and critical-role to the total revascularization response, as is suggested in the current data.

As such, there is a need to view the total contribution of both elements to the vascular remodeling process (i.e. angiogenesis and arteriogenesis) to understand the full impact on the reperfusion response to ischemia. The results presented here present one such example, but there likely exist multiple instances where such a view could be beneficial. For example, it was recently demonstrated knockout of Rac2 significantly impairs tissue reperfusion after FAL with a significant increase in muscle damage in the downstream ischemic tissue $(155,194)$. However, in one of the most detailed analyses of arteriogenesis to date, they show absolutely no effect on collateral remodeling that was sufficient to explain the deficits in perfusion or increased tissue damage (155). However, incorporating the known deficit in angiogenesis seen in Rac2 ${ }^{-/-}$mice may provide sufficient explanation for the total impairment in reperfusion response (194). A similar case can be made in the opposite direction. One example is the involvement of CD18/ICAM-1, where the documented impairment in arteriogenesis seen with blockade of ICAM 
(33) or enhancement with additional CD18 signaling (154) offers a strong alternative to or, at least, synergistic with explanation EPC mediated effects on angiogenesis alone in response to ischemia (195-197).

\section{Clinical Impetus for a Combined Role of Arteriogenesis and Angiogenesis in PAD}

These oversights may, in part, be explained by the different roles arteriogenesis and angiogenesis play during revascularization. Without sufficient arteriogenic remodeling, upstream resistance at the site of an arterial occlusion will remain high and limit the resolution of ischemia. However, it is important to note that improving bulk flow to the distal, ischemic tissue alone, as occurs during revascularization procedures, is insufficient to yield functional improvements or even tissue perfusion (183). Further, the functional outcomes induced by supervised exercise, one of the few Class IA recommendations for treatment of PAD (198), is preceded and correlated with angiogenic expansion within the ischemic muscle $(139,199)$. Together, these findings suggest that, even if microvascular remodeling does not contribute to the bulk flow into the ischemic tissue (see review (3)), microvascular function and capillary remodeling can play a significant role in the functional reperfusion within the distal tissue of PAD patients. By demonstrating that a selective impairment in angiogenesis alone can functionally contribute to the reperfusion process during hindlimb ischemia, the most widely used pre-clinical model to study the response to arterial occlusion, we hope to bridge the divide between looking at perfusion solely through the lens of arteriogenesis or angiogenesis. Rather, these data suggest

that to reach functional recovery and a full, healthy reperfusion response, it requires both a functional arteriogenesis and angiogenesis capacity. Therefore, clinical strategies that target both elements of vascular remodeling will likely yield the greatest therapeutic benefits for PAD.

Acknowledgements: The authors would like to thank the University of Virginia Research Histology Core for their work in histological tissue processing. 
Sources of Funding: This work was supported by American Heart Association awards 09PRE2060385 (JKM) and 10GRNT3490001 (RJP) and NIH grants T32-GM007267 (JKM), T32-HL007284 (JKM), and R01-HL074082(RJP).

Disclosures: The authors have no conflicts to report. 


\section{Chapter 3 Figures}

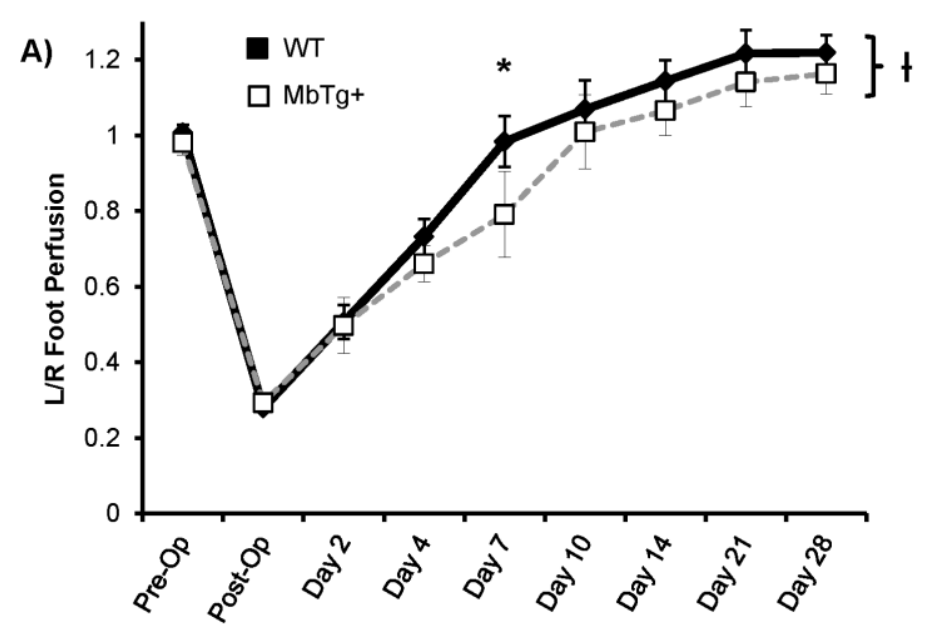

B)

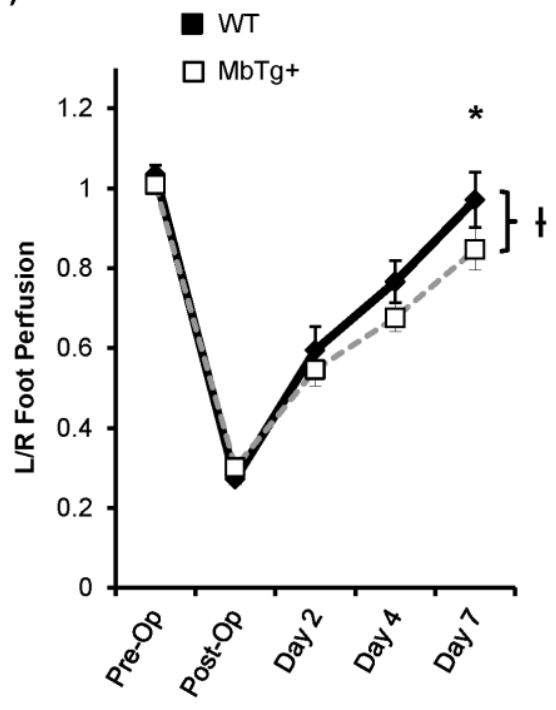

C)

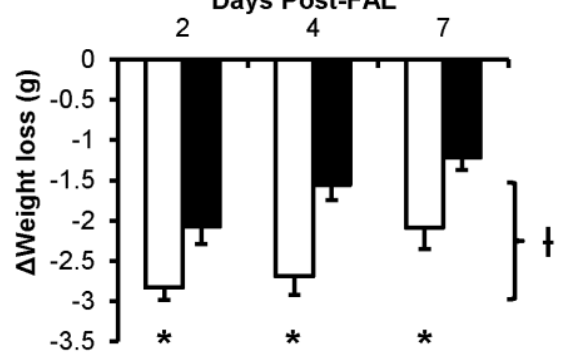

D)

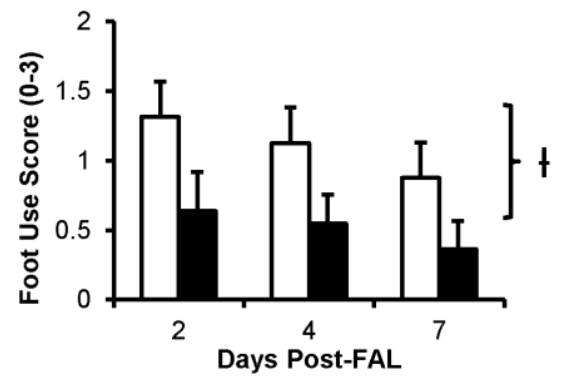

\section{Figure 3.1}

Myoglobin over-expression impairs perfusion recovery after FAL. A) Laser Doppler perfusion recovery curve (ischemic ligated leg, $\mathrm{L}$, normalized to non-ischemic sham, $\mathrm{R}$, leg) between $\mathrm{MbTg}^{+}$mice and WT littermate controls out to day 28 ( $n=9$ and 5 , respectively). B) Early perfusion recovery within the first 7 days post-FAL ( $n=14$ and 9 , respectively). Functional recovery as determined by post-FAL weight recovery $(\mathrm{C})$ and foot use (D) (scaled from 0 (normal) to 3 ). ${ }^{*}$ indicates $\mathrm{p}<0.05$ between $\mathrm{MbTg}^{+}$and WT within the given time point. + indicates $p<0.05$ between $\mathrm{MbTg}^{+}$and $\mathrm{WT}$ recovery curves across all time points. 

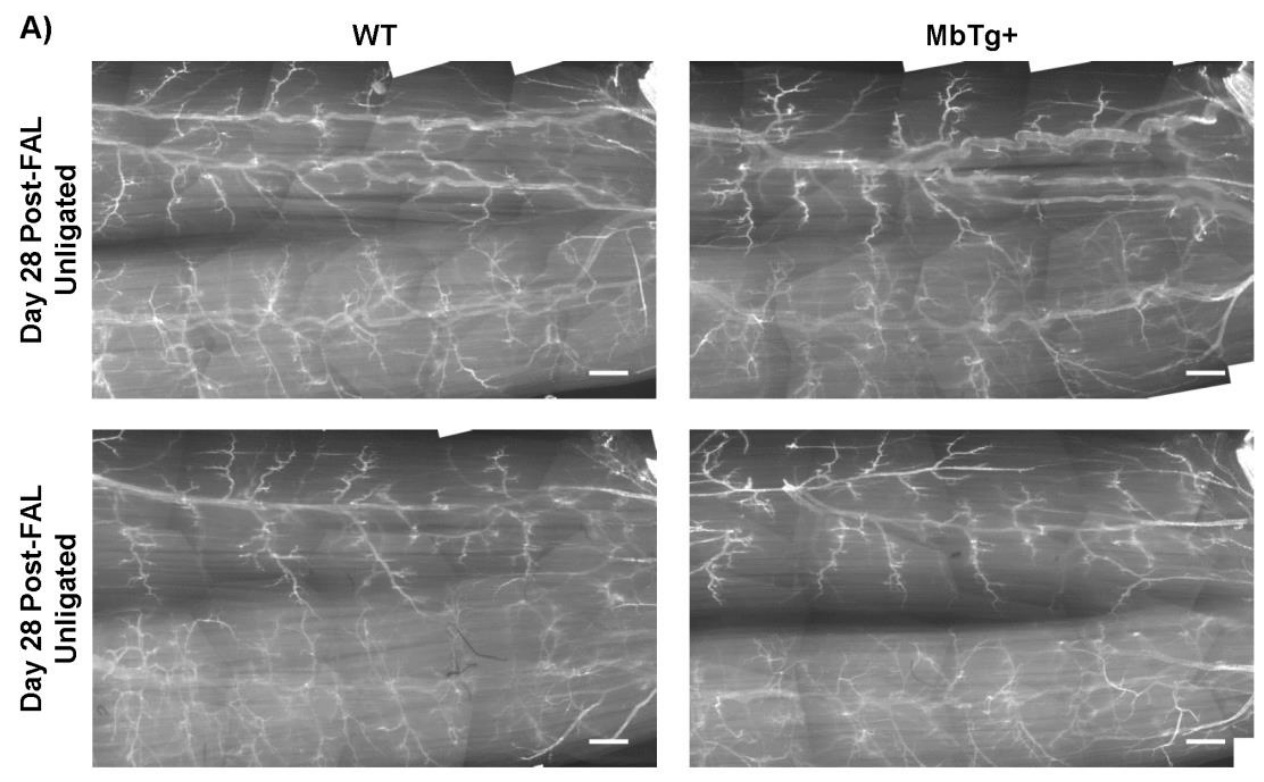

B)

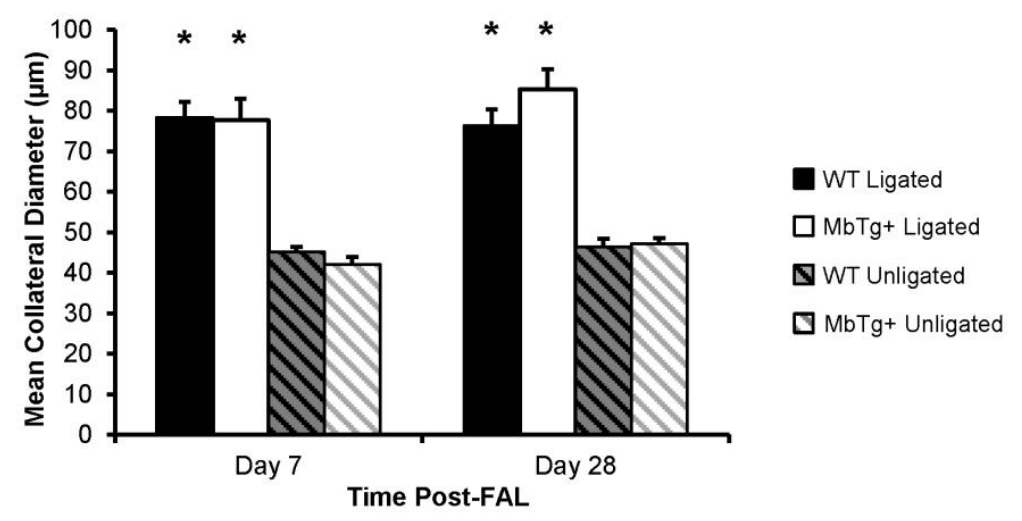

\section{Figure 3.2}

Collateral artery development in gracilis adductor muscle induced by FAL. A) A) Gracilis muscle whole mounts were fluorescently labeled using smooth muscle $\alpha$-actin to identify collateral arteries. Scale bars are $500 \mu \mathrm{m}$. B) Whole mount collateral artery diameters were quantified, showing outward remodeling of collateral arteries within the ligated limb starting at day 7 post-FAL, but no additional growth by day 28 post-FAL. There were no differences between $\mathrm{MbTg}^{+}$and WT mice $(\mathrm{n}=5$ and 4 , at day $7, \mathrm{n}=11$ and 7 , at day 28 , respectively). * indicates $p<0.05$ between ligated versus unligated limbs within $\mathrm{MbTg}^{+}$and WT mice. 

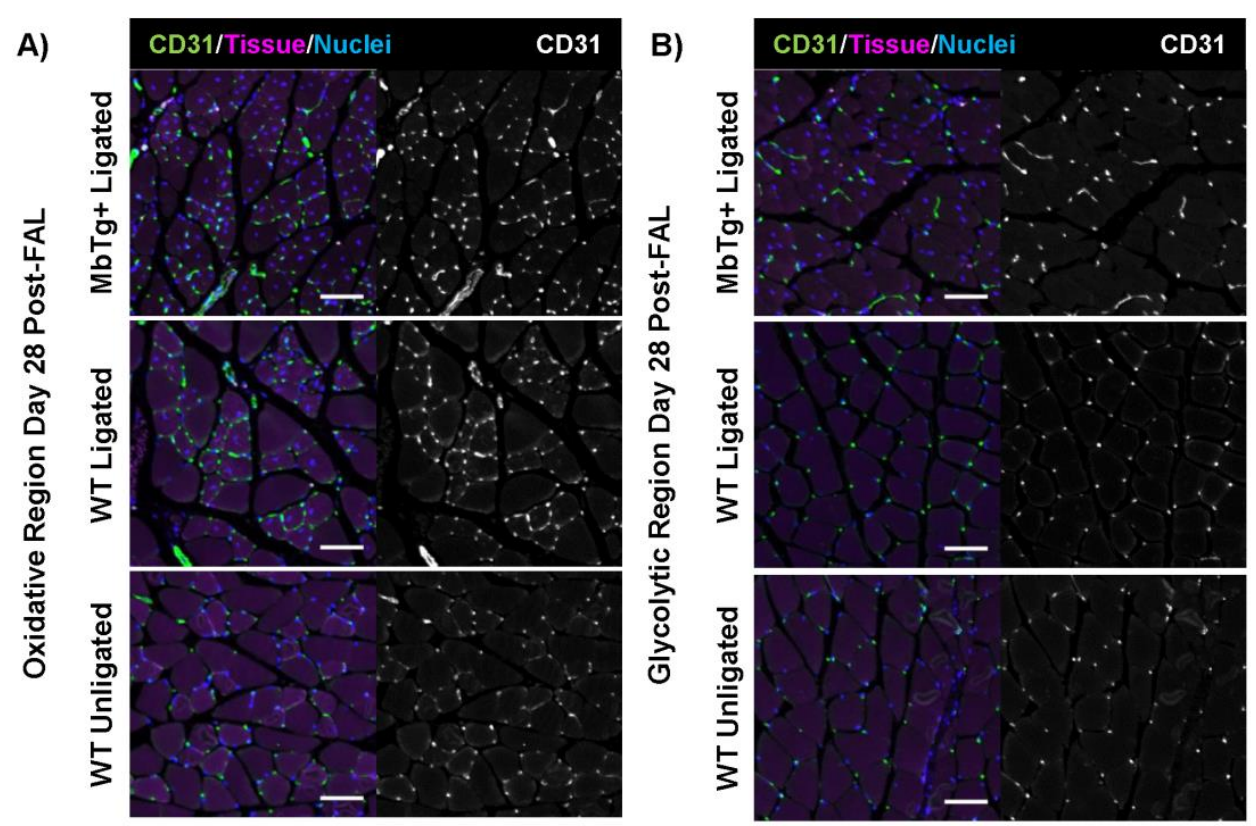

C)

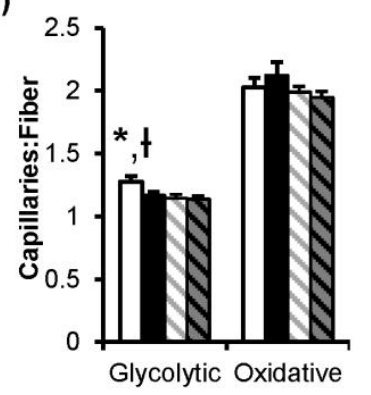

$\square$ WT Ligated
$\square \mathrm{MbTg}+$ Ligated
$\square \mathrm{WT}$ Unligated
$\square \mathrm{MbTg}+$ Unligated
D)

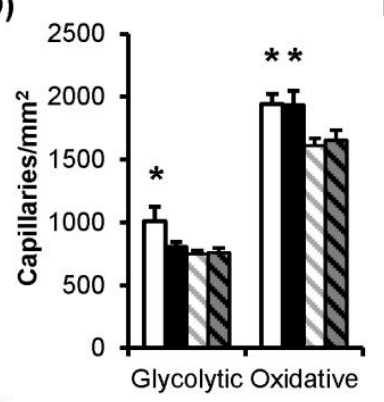

F)

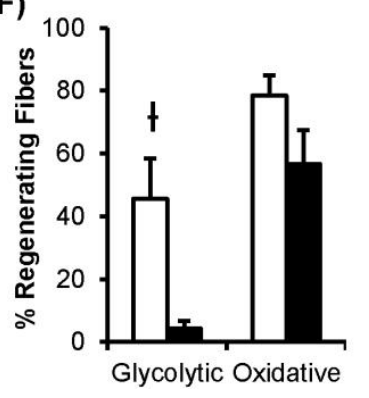

E)

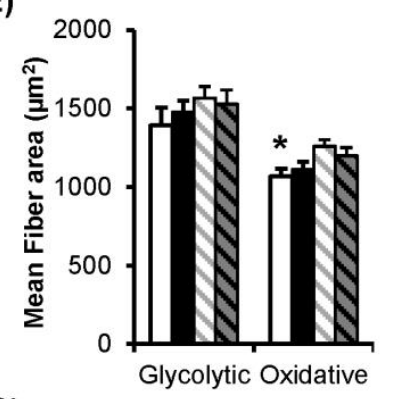

G)

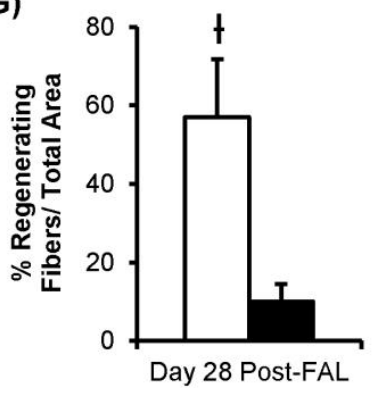

\section{Figure 3.3}

Capillary density within distal calf muscle cross-sections. A,B) Endothelial cells were labeled with CD31 and imaged in the oxidative (plantaris and deep gastrocnemius) and glycolytic (superficial gastrocnemius) regions of the calf muscle. C, D, E) Quantification of capillary to muscle fiber ratio (C), capillary density (D), and mean fiber cross sectional area (E) within ligated (solid) and unligated (hatched) limbs in $\mathrm{MbTg}^{+}$ (white) and WT (dark) control mice at 28 days post-FAL. F) The percentage of regenerating fibers within ligated limbs was quantified within the analyzed oxidative and glycolytic fields of view at 28 days postFAL. G) Percent of total cross sectional area within H\&E stained calf muscle cross-sections was also analyzed at 28 days post-FAL $\left(n=7, W T ; n=11, M^{2} \operatorname{Tg}^{+}\right)$. ${ }^{*}$ indicates $p<0.05$ between ligated and unligated muscles within $\mathrm{MbTg}^{+}$and WT mice. + indicates $\mathrm{p}<0.05$ between $\mathrm{MbTg}^{+}$and WT within ligated or unligated muscles. 
Chapter 4: Laser Speckle Flowmetry Method for Measuring Spatial and Temporal Hemodynamic Alterations Throughout Large Microvascular Networks.

Joshua K. Meisner, Suna Sumer, Kelsey P. Murrell, Timothy J. Higgins, and Richard J. Price. Microcirculation 19(7): 619-631, 2012. 


\section{Abstract}

Objectives: 1) Develop and validate laser speckle flowmetry (LSF) as a quantitative tool for individual microvessel hemodynamics in large networks. 2) Use LSF to determine if structural differences in the dorsal skinfold microcirculation (DSFWC) of C57BL/6 and BALB/c mice impart differential network hemodynamic responses to occlusion.

Methods: We compared LSF velocity measurements to known/measured velocities in vitro using capillary tube tissue phantoms and in vivo using mouse DSFWCs and cremaster muscles. Hemodynamic changes induced by feed arteriole occlusion were measured using LSF in DSFWCs implanted on C57BL/6 and BALB/c.

Results: In vitro, we found that the normalized speckle intensity (NSI) versus velocity linear relationship $\left(R^{2} \geq 0.97\right)$ did not vary with diameter or hematocrit and can be shifted to meet an expected operating range. In vivo, DSFWC and cremaster muscle preparations $\left(R^{2}=0.92\right.$ and 0.95, respectively) demonstrated similar linear relationships between NSI and centerline velocity. Stratification of arterioles into predicted collateral pathways revealed significant differences between $\mathrm{C} 57 \mathrm{BL} / 6$ and $\mathrm{BALB} / \mathrm{c}$ strains in response to feed arteriole occlusion.

Conclusions: These data demonstrate the applicability of LSF to intravital microscopy microcirculation preparations for determining both relative and absolute hemodynamics on a network-wide scale while maintaining the resolution of individual microvessels.

Keywords: laser speckle flowmetry, hemodynamics, network blood flow, intravital microscopy, microvascular imaging 


\section{Introduction}

The proper maintenance of microvascular network structure and vasoactivity is essential for normal tissue physiology and function both in the short term (i.e. via smooth muscle tone) and long term (i.e. via remodeling of vascular wall structure) (200). Vascular wall shear stress is generally thought to play a central role in coordinating the sequence of events leading to arteriolar remodeling $(151,200,201)$. However, few studies are aimed at determining the molecular mechanisms of coordinated arteriolar remodeling in response to shear stress, even in light of its clinical significance to hypertension, peripheral arterial disease, tumor vascularization, and retinopathy. One major reason for why mechanistic investigation in this important field has been limited is that experimental approaches capable of quantitatively linking shear stress changes to arteriolar remodeling in individual vessels over time throughout large microvascular networks are widely unavailable, limiting many of the few existing studies to computational approaches or single time points $(201,202)$. Here, we define a network as the interconnected structure of all microvessels from feeding arterioles to draining venules in the visible microvascular preparation.

One potential method for quantitatively measuring network autoregulation is laser speckle flowmetry (LSF). Laser speckle imaging works on the principle that coherent light (i.e. laser light) creates a random interference (speckle) pattern when shone on a static tissue region, with reduced contrast in regions exhibiting motion. Initially proposed in 1981, Fercher and Briers (203) showed that, using spatial statistics, it is theoretically possible to create a 2dimensional distribution map of velocity based on the dynamic motion of scattering particles in a tissue (see reviews $(204,205)$ ). With the technological advances in CCD and CMOS cameras, as well as improvements in statistical algorithms for enhanced temporal and spatial resolution $(204,206,207)$ and processing power $(208)$, the LSF technique has become readily accessible with a demonstrable ability to produce distribution maps of blood flow in the microcirculation 
(e.g. pial circulation (209-212), mesentery (213), dorsal skinfold window chamber $(206,214,215)$ and retina (216)). Therefore, LSF presents a clear opportunity to quantify hemodynamics at the resolution of the microcirculation.

To date, however, laser speckle imaging has been largely limited to use as a qualitative tool of regional perfusion $(204,209-212)$, even when correlated to microvascular remodeling (217). One of the key factors leading to this limitation in current applications is the use of lowmagnification optical components that are needed to capture an entire network within a single field of view. This limits the ability to resolve the majority of components of the microcirculation. This limitation has been historically overcome using intravital microscopy techniques in twodimensional networks to observe the microcirculation at high magnification. A second key issue is that, of the few studies specifically suggesting the potential of laser speckle imaging in the microcirculation $(204,213,215)$, there has been a lack of validation in vivo, which is of particular concern given the debated quantitative potential of speckle in vivo (see review (218)). Therefore, we tested and validated the use of LSF with intravital microscopy of the mouse cremaster muscle and dorsal skinfold window chamber using particle imaging velocimetry of circulating fluorescent microspheres. Subsequently, we sought to demonstrate the potential of LSF toward quantifying changes in hemodynamics throughout the dorsal skinfold window chamber. Specifically, the potential of LSF to quantify network hemodynamics was applied to test how strains known to have large differences in microvascular network structure (i.e. C57BL/6 and BALB/c $(68,116,142))$ acutely respond to changes in network flow. 


\section{Method and Materials}

\section{Animals}

All animal studies were approved by the Animal Research Committee at the University of Virginia and conformed to the AHA Guidelines for the Use of Animals in Research. C57BL/6 and BALB/c male mice, 8-10 weeks of age, were purchased from Charles River Laboratories (Wilmington, MA). "n" values for each experiment are provided in the figure legends.

\section{Dorsal Skinfold Window Chamber Implantation for Intravital Imaging}

Window chamber surgeries were performed in a similar procedure to that previously described by our laboratory (90). Briefly, wild type mice were anesthetized by i.p. injection of ketamine, xylazine, atropine, and saline (100/20/0.4 mg/g body weight), with supplemental doses equal to one fifth of the initial dose administered as required. After anesthesia, the dorsal skin was depilated and sterilized. Using a dissecting microscope, one half of a dorsal skinfold window chamber was surgically attached. A $12 \mathrm{~mm}$ diameter circular section of the entire top skin tissue layer and subcutaneous tissue was excised under sterile conditions to expose the microcirculation of the underside of the dermis in the opposite layer skin with a goal of minimizing damage to the microvascular network. The second half of the window chamber was installed, and the exposed network was filled with sterile Ringer's solution and covered with a sterile glass coverslip that inserts into the titanium frame. Buprenorphine was administered by subcutaneous injection post-operatively for analgesia $(0.3 \mathrm{mg} / \mathrm{g}$ body weight). After the end of the experiment, the mouse was euthanized with an overdose of pentobarbital.

\section{Cremaster Muscle Preparation for Intravital Imaging}

The cremaster muscle was isolated similar to a previously described method (219). Briefly, C57BL/6 wild type mice were anesthetized by i.p. injection of ketamine, xylazine, 
atropine, and saline (100/20/0.4 mg/g body weight), with supplemental doses equal to one fifth of the initial dose administered as required. After anesthesia, the scrotal sac was cut longitudinally and the testis was gently pulled out and cleared of connective tissue to expose the cremaster muscle. Tissue moisture was maintained with warmed sterile Ringer's solution. Next, the muscle flap was opened with a longitudinal incision along the anterior wall of the muscle, taking care to avoid the primary feeding arteriole and venule pair. After incision, the testis was returned to the abdominal cavity and the animal was secured to a heated, costume-designed Plexiglas stage. The muscle flap was then stretched and secured over an imaging window by 30 gauge needles. The stage was then mounted to the microscope. At the end of the experiment, the mouse was euthanized with an overdose of pentobarbital.

\section{Laser Speckle Flowmetry for Imaging of Microvascular Network Flow}

When coherent light, such as that from a laser, illuminates a tissue surface, it produces a consistent scatter pattern known as speckle. This pattern is characteristic for a given tissue structure. As elements within the tissue move (i.e. red blood cells) and scatter light differently, the speckle pattern fluctuates. When these fluctuations are integrated over time, such as during the acquisition of a digital image, the image shows a decrease in speckle contrast and variation due to the averaging of the intensity of each speckle. Using spatial statistics of time-integrated speckle contrast, Fercher and Briers (203) showed that this decrease in speckle contrast ( $C$, Eq. 1) can be related to particle flux, where $\sigma$ is the standard deviation and $/$ is the mean of the pixel (Pij, Figure 4.1) values in a $7 \times 7$ neighborhood. For our calculations, we used a simplified algorithm (Eq. 2) for relating speckle contrast $(C)$ and exposure time ( $T$ ) to autocorrelation time $(\tau)$, which is directly proportional to particle velocity ( $S I$, speckle intensity) within the local volume of the optical tissue slice. This simplified algorithm removes the assumption of Gaussian or Lorentzian distributions, and is valid for the flow ranges seen in microvascular networks (e.g dorsal skinfold window chamber) (206). 


$$
C_{i j}{ }^{2}=\frac{\sigma_{i j}^{7 x 7^{2}}}{\left\langle I_{i j}^{7 x 7}\right\rangle^{2}}(1) \quad \frac{1}{\tau}=\frac{1}{2 T C^{2}}=L S I \text { (2) }
$$

In our arrangement, a beam expanded 30mW, 658nm laser diode (Newport LPM658-30) was used to illuminate the window chamber tissue (Figure 4.1). The raw laser speckle images were collected by an intravital microscope (Zeiss Axioskop,Thornwood, NY) using a 4x air lens (Zeiss Acroplan LD NA=0.1) and acquired and digitized by a cooled, monochrome CCD camera (Optronics Quantifier, Goleta, CA) $(7.4 \mu \mathrm{m} \times 7.4 \mu \mathrm{m}$ pixels). The selection of the speckle imaging setup was chosen to balance the resolution of the scope and size of the speckle. During imaging, the speckle of light is projected into the CCD camera at a size (S) of $S=2.44 \lambda(1+M) /(2 N A)$, where $\lambda, N A$, and $M$ are the wavelength of laser light, the numerical aperture of the objective, and the magnification of the objective, respectively. At these settings, the physical size of the CCD pixel element satisfied the Nyquist sampling criteria of at least 2 pixels per individual speckle (220). At each field of view, a sequence of $\geq 15$ 12-bit raw speckle images were acquired at variable exposure times to capture average flow. These raw speckle images were converted to laser speckle flow index maps using a MATLAB algorithm. To account for variations in tissue background motion from mouse movement, the processed flow images were normalized to median background intensity and then analyzed using ImageJ software (NIH, Bethesda, MD) to quantify the average speckle flow intensity. Individual network flow images were then merged into larger, 2D microvascular network maps using Adobe Photoshop (CS2, Adobe Systems Inc, San Jose, CA). For dorsal skinfold window chamber microligation experiments, brightfield and processed speckle flow images were merged into separate color panes before montaging, allowing for precise overlay of brightfield and speckle flow network montages. 


\section{In Vitro Speckle Tissue Phantom Imaging Protocol}

To generate light scattering tissue phantoms, titanium dioxide nanopowder (677469, Sigma Aldrich, St Louis, MO) was sonicated and dispersed into 184 Sylgard PDMS gel (Dow Corning, Midland, Ml) at $1 \mathrm{mg} / \mathrm{mL}$ and allowed to cure overnight. Polyethylene tubing was connected to glass capillary tubes of varying inner diameters and similar wall thicknesses (142, 282, and 447 $\mu \mathrm{m}$, Drummond Microcap, P1799 Sigma Aldrich, St Louis, MO) and placed atop the phantom to mimic a superficial vessel in a scattering tissue medium. The tubing was connected to a PHD2000 syringe pump (Harvard Apparatus, Holliston, MA) which was used to drive a pre-determined, randomized program of flow rates of defibrinated bovine blood (Hemostat Labs, Dixon, CA). Pump rate and image acquisition were controlled by customdesigned LabVIEW software (National Instruments, Austin, TX). Each complete program of flow rates was repeated 5 times at a specific exposure time, hematocrit, or tubing diameter. For hematocrit variation, the defibrinated blood was centrifuged at $800 \mathrm{~g}$ for $10 \mathrm{~min}$ and then resuspended to the initial $36 \%$ hematocrit or concentrated by removal of plasma and resuspended to $54 \%$ hematocrit. A logarithmically spaced distribution of velocities was used to determine how variation in exposure time (3ms and $20 \mathrm{~ms}$ ) alters the dynamic range of the linear speckle velocity versus absolute velocity relationship.

\section{In Vivo Calibration Imaging Protocol Using Laser Speckle and Microspheres}

To determine the relationship between speckle flow measurements and absolute measurements of hemodynamics, circulating fluorescent microspheres were used as tracer particles. Cremaster and window chamber tissues were prepared for intravital imaging, laser speckle flowmetry, and fluorescence microscopy as described above. Both preparations were drip superfused with warmed, sterile Ringer's solution throughout the duration of imaging. A $2 \%$ wt/vl green fluorescent microsphere solution (3.0 $\mu \mathrm{m}$, Fluoro-Max G0300, Thermoscientific, Waltham, MA) was continuously injected intravenously via tail vein catheter at a variable rate 
$(<200 \mu \mathrm{L} / \mathrm{hr})$ to ensure continuous observation of $\geq 2$ discrete microspheres per frame in the observed microvessels. At least 5 random fields of view per preparation were chosen for imaging. At each field, a 20 second sequence of $100 \mathrm{~ms}$ exposure images were taken under $100 \mathrm{~Hz}$ pulsed LED (M455L2-C4, Thorlabs, Newton, NJ) fluorescent epillumination to allow for microsphere tracking. This was immediately followed by the acquisition of a $\geq 15$ frame sequence ( 4 frames per second) of raw speckle images ( $<5$ seconds switch procedure).

\section{In Vivo Measurement of Altered Network Flow Using Laser Speckle}

To determine how arteriole occlusion affects flow throughout large microvascular networks with different degrees of arteriolar interconnectivity, wild-type BALB/c and C57BL/6 mouse strains were implanted with dorsal skinfold window chambers as described above. After $24 \mathrm{hrs}$ of post-surgical recovery, mice were induced and maintained under $1.5 \%$ inhaled isoflurane anesthesia and prepared for intravital imaging on a warmed microscope stage. After a 15 minute equilibration period, the entire window chamber was imaged by brightfield microscopy and laser speckle flowmetry with the glass coverslips left in place. At each field of view, imaging alternated between brightfield image acquisition using a using $560 \mathrm{~nm}$ bandpass filter to improve vessel contrast and laser speckle imaging without transillumination or a bandpass filter. A 20ms exposure time was chosen for all imaging steps based on initial experiments. After the initial imaging step, the glass coverslip window was removed and the microcirculation was exposed and drip superfused with warmed, sterile Ringer's solution. After exposure, the largest feeding arteriole to the window chamber network with adequate separation from its paired venule was chosen for occlusion using a custom designed and fabricated micro-cauterization device capable of isolated cauterization down to an 100 micron diameter. The tissue was then flushed with sterile Ringer's solution and a sterile glass coverslip was replaced and secured over the window chamber. Inhaled isofluorane was removed, and the mouse was then allowed to recover for 24 hours. After 24 hours, the mouse was, again, induced 
and maintained on inhaled isofluorane. After a 15 minute equilibration period, the entire window chamber was reimaged as before with the glass coverslip left in place to generate a second set of brightfield and laser speckle images without a change in laser illumination orientation. Together, these datasets represent the initial hemodynamic state of the window chamber network and the acute (24 hours post-occlusion) hemodynamic state of the entire network in response to the altered network structure and flow patterns induced by the primary feeding arteriole occlusion.

\section{Microsphere Particle Velocity Analysis}

To determine hemodynamics using fluorescent microsphere tracer particles, centerline velocity was measured by determining the distance traveled by individual spheres during the observed number of pulsed time steps $(10 \mathrm{~ms}$ at $100 \mathrm{~Hz}$ LED strobe rate, Figure $4.3 \mathrm{~A})$. Within individual frames, only the fastest moving spheres in the center of the microvessel segment were chosen for analysis to exclude slower microspheres near the vessel wall above and below the central axis that were also visible in the focal plane. A typical sequence of images in a given field of view yielded $>3$ arteriole or venular segments. Only vessel segments with $\geq 6$ measurable centerline particles during the complete sequence were included in the analysis to ensure an accurate representation of average centerline velocity. While velocity analysis was limited to centerline velocity due to optical clarity and the number of particles visualized during acquisition, centerline velocity represents a useful approximation for mean velocity in microvessels $(221,222)$.

\section{Laser Speckle Flowmetry Analysis}

For individual microvessel segments, average speckle pixel intensity was measured along a length of the given segment across the full diameter of the vessel. A similarly sized region of the neighboring background tissue was also measured for speckle intensity in each 
field of view or montage separately for each vessel segment. The laser speckle intensity of the vessel $\left(S I_{\text {vessel }}\right)$ was normalized (NSI, normalized speckle intensity) to the neighboring background intensity $\left(S I_{\text {background }}\right)$ to remove background artifact from general tissue motion and variations in speckle illumination according to Eq 4. With the assumption of Poiseuille flow, wall shear rate is proportional to average blood velocity divided by tube diameter. Therefore, proportional wall shear rate (SSR, speckle shear stress) was calculated using the NSI measurement of average blood velocity and vessel diameter (D) (Eq 4).

$$
N S I=\frac{L S I_{\text {vessel }}}{L_{\text {background }}}-1(3) \quad S S R \propto N S I / D^{(4)}
$$

Within the cremaster network, individual fields of view containing the input $\left(Q_{\text {parent }}\right)$ and output $\left(Q_{\text {daughter }}^{A}\right.$ and $Q_{\text {daughter }}^{B}$ flows at bifurcations were used to assess conservation of mass. To compare NSI and absolute velocity accuracy, relative error from perfect conservation of mass (Eq 5) was calculated from flow estimates for LSF ( $\left.Q_{\text {speckle }}\right)$ using $\mathrm{NSI}$ as a measurement of average velocity (Eq 6) and flow estimates using absolute microsphere centerline velocity ( $\left.V c_{\text {sphere }}\right)$ (Eq 7, assumed Poiseuille flow, where $Q_{\text {sphere }}$ is flow).

$\operatorname{Error}(\%)=\frac{\left(Q_{\text {daughter }}^{A}+Q_{\text {daughter }}^{B}\right)-Q_{\text {parent }}}{Q_{\text {parent }}} * 100$

$Q_{\text {speckle }} \propto N S I * D^{2}($ a.u. $)(6)$

$Q_{\text {sphere }} \propto V c_{\text {sphere }} * D^{2}$ (a.u.) (7) 


\section{Network Structure and Flow Analysis}

Using a custom-designed LabVIEW program (available on request), the montaged network images composed of brightfield and speckle velocity data were used to measure diameter and NSI for all arteriole segments in a given window chamber network visible in both the pre-occlusion and post-occlusion microvascular networks. Arteriole segments were defined as the length of vessel between arteriolar branch points. Prior to analysis, all pre- and postocclusion network pairs were de-identified and randomized. Arcade vessels were defined either as circular, continuous connections of arterioles or venules larger than terminal arterioles or as connections between primary feeding arterioles or draining venules through non-terminal arteriole or venules, respectively. For micro-occlusion experiments to quantify changes in network hemodynamics, individual arteriole segments were classified into "collateral" or "background" in de-identified images. The two most direct and largest continuous diameter arteriole pathways connecting primary feeding arterioles (either self or separate) to the downstream half of the occluded arteriole were termed "collateral" pathways (see Figure 4.5 for examples). All arteriole branch segments in this pathway were deemed "collateral" segments, all other arterioles in the network that were not designated as "collateral" segments were designated as "background" arteriole segments.

\section{Statistics}

For comparison of the distribution of pre-occlusion arteriolar diameter and the percent change in velocity (NSI) and shear rate (SSR) per arteriolar segment (NSI) in BALB/c and C57BL/6 networks, segment diameters and percent change in velocity or shear stress were binned and averaged across all mice within the group. Linearity of the NSI versus known velocity was tested using linear regression. Regression was performed on logarithmically transformed data for in vitro exposure variation analysis using a log distribution of velocity steps. All data were reported with error bars representing \pm standard deviation of the mean. Two-way 
ANOVA statistical testing combined with the Holm-Sidlak method for multiple testing of interactions was used to compare differences in vascular loop structure and change in flow within collateral and background arterioles for BALB/C and C57BL/6 mice. Significance was assessed at $p<0.05$ (Sigma Stat 3.4, Systat Software, San Jose, CA).

\section{Results}

\section{In Vitro Testing of Laser Speckle Flowmetry Linear Response}

During in vitro tissue phantom testing, the normalized speckle index (NSI) exhibited a highly linear relationship with average blood velocity $\left(>0.97 R^{2}\right.$, Figure $4.2 A, n=6$ trials per velocity step at each diameter, 3ms exposure). This linear relationship has no significant variation with diameter spanning from 142 to $447 \mu \mathrm{m}$ for glass capillary tubes (Figure $4.2 \mathrm{~A}$ ). Further, this linear relationship has no significant variation with respect to hematocrit when comparing $\mathrm{NSI}$ to velocity using $36 \% \mathrm{PCV}$ and 54\% PCV substrate blood (Figure 4.2B, $\mathrm{n}=6$ trials per velocity step per hematocrit, 3ms exposure). As previously demonstrated (214), altering camera exposure time adjusts the range and sensitivity of the linear operating region of the $\mathrm{NSI}$ to velocity relationship (Figure $4.2 \mathrm{C}$ ). For a $20 \mathrm{~ms}$ exposure time, the linear operating range spans from zero to approximately $1 \mathrm{~mm} / \mathrm{s}$ average velocity with sensitivity down to a minimal resolution of $0.05 \mathrm{~mm} / \mathrm{s}\left(0.92\right.$ log-weighted $R^{2}$ for region to $1 \mathrm{~mm} / \mathrm{s}, \mathrm{n}=6$ trials per velocity step). For a shorter 3ms exposure time, the linear operating range of the NSI to velocity relationship increases, spanning from zero to greater than $20 \mathrm{~mm} / \mathrm{s}$ average velocity. However, sensitivity decreases to a minimal resolution of $0.2 \mathrm{~mm} / \mathrm{s}\left(0.90 \mathrm{log}\right.$-weighted $\mathrm{R}^{2}$ for region from $0.2 \mathrm{~mm} / \mathrm{s}, \mathrm{n}=6$ trials per velocity step).

\section{In Vivo Validation of LSF Measurements}

Velocity measurements were collected in cremaster and dorsal skinfold window chamber (DSFWC) preparations using circulating fluorescent microspheres and NSI. In the mouse 
cremaster muscle, a highly linear relationship existed between absolute velocity as measured by fluorescent microspheres and NSI (Figure 4.3). This linear relationship was true both within a given muscle $\left(R^{2}=0.66 \pm 0.15, n=4\right.$ cremaster muscles $)$ and across multiple muscles $\left(R^{2}=0.95\right.$ when binned by $0.1 \mathrm{~mm} / \mathrm{s}$ increments of absolute velocity, $\mathrm{n}=4$ muscles, Figure $4.3 \mathrm{C})$. In cremaster muscle, mass balance of flow from parent to daughter vessels was used as an additional verification of accuracy in both particle velocimetry and $\mathrm{NSI}$ measurements. NSI measurements showed a similar degree of mass balance error $(-6.06 \pm 0.04 \%$ S.E.M. $n=14$ bifurcations in $\mathrm{n}=4$ muscles) compared to circulating microsphere measurements (10.03 \pm 0.07\% S.E.M., no significant differences versus NSI estimates). These similar errors suggest a compounding of error from both $\mathrm{NSI}$ and microsphere velocity measurements in the binned data. In the DSFWC, there was a similar degree of linearity of LSI to absolute microsphere velocity measurements within individual preparations $\left(R^{2}=0.48 \pm 0.16, n=5\right.$ windows $)$ and across preparations $\left(R^{2}=0.92\right.$, when binned by $0.1 \mathrm{~mm} / \mathrm{s}$ increments of absolute velocity, $\mathrm{n}=5$ windows, Figure 4.3E). Interestingly, there was similarity in the NSI versus absolute microsphere velocity slope between cremaster and DSFWC data.

\section{Application of LSF and Brightfield Intravital Imaging to Large Microvascular} Networks

As demonstrated in Figure 4.3D/F, individual fields of view for both brightfield and LSF can be stitched together to yield high resolution maps of vascular structure and blood velocity in both cremaster and DSFWCs. Both cremaster and DSFWC networks demonstrate measurable LSF velocity signal in arterioles $<15 \mu \mathrm{m}$ and venules $>250 \mu \mathrm{m}$ in diameter (Figure 4.3D/F). This resolution allowed for an average of 34.6 arteriole LSF measurable segments per $\sim 80 \mathrm{~mm}^{2}$ of DSFWC tissue. An identical intravital microscopy LSF method was applied to several other intravital preparations including the mouse ear skin and exposed hindlimb skeletal muscle yielding similar results (data not shown). 


\section{Alteration in Network and Collateral Pathway Hemodynamics in BALB/c and C57BL/6 Mice}

We used a microcauterization device to occlude a primary feeding arteriole in order to determine how the differences in microvascular network structure and function between BALB/c and C57BL/6 mouse strains (Figure 4.4A/B) alters flow redistribution in response to the loss of a primary feeding arteriole. As previously demonstrated in other organs, C57BL/6 and BALB/C DSFWC microvascular networks showed significant differences between the strains $(68,116,142)$. There was a skewing of the BALB/c networks to a higher percentage of small diameter arteriole segments and fewer arteriolar arcade loop structures compared to the C57BL/6 networks (mean pre-ligation arteriole segment diameter; BALB/C $=27.2 \pm 7.3 \mu \mathrm{m}$, $\mathrm{n}=163$ segments in 5 mice: $\mathrm{C} 57 \mathrm{BL} / 6=36.8 \pm 11.2 \mu \mathrm{m}, \mathrm{n}=183$ segments in 5 mice, Figure 4.4C/D). However, in converse to the arteriole loops, there was an increase in venular loop structures in the BALB/c networks compared to the C57BL/6 networks (Figure 4.4D).

Given the significant differences in network structure, we tested whether this would translate into significant hemodynamic differences throughout the network in response to the occlusion of a primary arteriole. LSF was used to quantify hemodynamic changes from pre to 24hrs post primary feeding arteriole occlusion. This was done either independent of network context or with stratification into "collateral" and "background" arteriole segments (Figure 4.5). When analyzed across an entire network, there were no significant differences in the distribution of velocity or shear stress changes from pre- to post-arteriole occlusion. However, when vessel segments were stratified into likely collateral segments or background arteriole segments, there was a significant percent increase in velocity and shear stress in collateral segments compared to background arterioles $(p<0.023$ and $p<0.045$, respectively, Figure $4.5 B)$, but this was only significant within the C57BL/6 strain ( $p<0.017$ and $p<0.001$, respectively, Figure 4.5B). 


\section{Discussion}

Both short and long term autoregulatory responses are the result of the spatial and temporal integration of multiple signals arising from within both the microvascular network and surrounding tissue. In particular, hemodynamic signals are known to be critically important, but they are difficult to characterize in individual microvessels through time. Thus, the ability to obtain hemodynamic information (i.e. velocity, flow, and/or shear stress) on an individual vessel level across entire microvascular networks could represent an important advance in our understanding of integrated "whole-network" microvascular function. However, such measurements are technically challenging due to limitations on resolution, optical clarity, total network area, or duration of experiment. Here, we demonstrate that laser speckle flowmetry is capable of resolving blood velocity across the arteriolar and venular spectrum, on an individual microvessel level, throughout entire microvascular networks. Further, our data validate that the acquired velocity data from laser speckle imaging is quantitative in nature for blood velocity within and across individual intravital preparations, a question that has been debated previously (218). Of particular interest for the microcirculation, our in vitro testing additionally demonstrates that the laser speckle velocity measurements are independent of vessel diameter and hematocrit, which can vary extensively in the microcirculation (see Figure 4.4). When taken together, these results indicate that laser speckle flowmetry is a powerful tool for measuring hemodynamics in individual vessels in intact microvascular networks.

\section{Laser Speckle Flowmetry as a Quantitative Tool for Absolute Measurements}

The potential ability of LSF to measure absolute velocity is currently under debate $(205,218)$. Here, our empirical studies suggest that laser speckle can be used to quantitatively determine changes in microvascular velocity and flow at the level of an individual vascular segment. Various laser speckle flowmetry approaches have been used previously for the 
measurement of hemodynamics in a variety of intravital microscopy models, most prominently in the cerebral circulation (see review (205)), but also in the dorsal skinfold window chamber $(206,214,215)$, mesentery (213), and ear circulation (217). However, in contrast to our approach, these studies almost exclusively assessed global or regional changes in tissue perfusion (205), and in vivo validation of laser speckle imaging data was extremely limited. We believe the primary reason for the focus on global and regional perfusion is related to the use of a single "wide field" acquisition to capture the entire network at once. This approach is exemplified in a recent study by Rege et al (217) to equate global and regional alterations in hemodynamics with alterations in vascular structure. Our data on differential C57BL/6 and BALB/c microvascular responses suggest that critically important information can be extracted when hemodynamics are examined on an individual vessel basis.

\section{Advantages of Laser Speckle Imaging}

Laser speckle flowmetry applied to intravital microscopy provides several advantages to current techniques available for measuring hemodynamics in the microcirculation. First, LSF has high enough resolution to image individual vessels. This stands in contrast to the bulk perfusion measurements acquired by laser Doppler perfusion imaging $(160,211,223)$ and wide field applications of $\operatorname{LSF}(205,211,217)$. Second, where high resolution is available, such as in the recent development of optical imaging techniques including optical coherent tomography $(224,225)$, intravital microscopy of fluorescent particles (226-228), and multiphoton microscopy $(229,230)$, there are often limitations with respect to spatial coverage and the challenges of registering multiple fields of view. A similar difficulty is present in the more traditional microvascular tools of autocorrelation and dual-slit velocimeters $(231,232)$ in the experimental duration needed to capture hemodynamics throughout an entire network one vessel at a time. Lastly, LSF is easily integrated into an existing intravital microscopy set up, with low cost and minimal modification. This is in contrast to photoacoustic microscopy, optical coherence 
tomography, and multiphoton microscopy, which require expensive, uniquely dedicated equipment for data collection and often require extensive custom development $(224,229,230,233,234)$.

Our results also demonstrate the adaptability of LSF for exploring microvascular network hemodynamics. Specifically, our results on sensitivity and linear dynamic range of LSF demonstrate that variations in camera exposure time can be used to adapt a given LSF setup to the expected velocity range within a specific intravital preparation, as has been suggested previously (214). This would allow for imaging of both low velocity (e.g. in the dorsal skinfold window chamber) and high velocity (e.g. collateral vessel flow in upper hindlimb muscle (data not shown)) intravital preparations. Interestingly, in the two intravital microscopy models of the microcirculation tested here, both setups yielded surprisingly similar in vivo validation relationships. This suggests that LSF should be applicable to a wide variety of intravital preparations and, potentially, there may be a broadly applicable relationship between NSI and absolute velocity across multiple intravital preparations, though this would require additional study. Moreover, although the current study focused on using a spatial algorithm for determining the speckle velocity measurement, it is well documented that the mode of acquisition and speckle algorithm processing can be readily modified to balance for further increases in spatial resolution versus temporal resolution (see review (204)). Though not examined in the current study, further spatial resolution may also be achieved with the use of higher numerical aperture microscope objectives (220), potentially allowing for direct measurement of wall shear rate. Together the adaptable linear range, potentially broad applicability, and ability to readily adjust for increased spatial or temporal resolution demonstrate how LSF can be readily adapted and customized to a large range of microvascular studies on network regulation and hemodynamics. 


\section{Use of LSF for Interrogating Network Responsiveness to Microvessel Occlusion in $\mathrm{C} 57 \mathrm{BL} / 6$ and $\mathrm{BALB} / \mathrm{c}$ Mice.}

In the current study, we sought to demonstrate the power of LSF and individual vessel hemodynamic analysis by examining the responsiveness of two structurally different microvascular networks to primary feeding arteriole occlusion. BALB/c and C57BL/6 mice represent opposite ends of a spectrum of arteriolar connectivity and network structure across inbred mouse strains within the pial (68) and skeletal muscle circulations (116). Here, we demonstrated that similar differences exist for skin microcirculation, as evidenced by the shifted distribution of arteriolar segments within BALB/c windows toward fewer large arteriole segments (Figure 4.4C), along with a decreased number of anastomotic arcade arterioles (Figure 4.4D). Interestingly, BALB/c mice exhibited more venular arcade pathways when compared to C57BL/6 mice, an observation not previously reported. While the functional significance of this finding is unknown, a recent study demonstrates that such pathways can have a functional significance in upstream capillary perfusion (235). Together, the data presented here further demonstrate a significant difference in network structure between BALB/c and C57BL/6 mice.

Combining the confirmed difference in network structure between the two strains with the utility of network wide laser speckle flowmetry, we were able to test whether differences in network structure and vasoactivity in $\mathrm{C} 57 \mathrm{BL} / 6$ and $\mathrm{BALB} / \mathrm{c}$ mice create differences in responsiveness to primary arteriole occlusion. Our data show that, when viewed on a networkwide scale, there were few apparent differences in how blood flow is redistributed. This was a somewhat unexpected finding given the well documented differences in perfusion deficits between the two strains upon larger vessel occlusion, such as the femoral artery $(116,142)$ or middle cerebral artery (68). However, when analyzed on an individual vessel level using network structure as a guide, our data demonstrated that C57BL/6 mice recruit their predicted collateral vessels more efficiently upon primary arteriole occlusion, as denoted by significant 
increases in velocity and shear stress in collateral segments (Figure 4.5B). It is important to consider that the observed differential recruitment of collateral arterioles may be a significant factor contributing to the differential capacity to undergo collateral remodeling between the two strains. Though not directly tested, the differences in network structure alone between the two strains may underlie the differential collateral recruitment given that there are no significant differences in conduit artery (236) or arteriolar vasoreactivity (237) between C57BL/6 and BALB/c strains.

While the rules guiding our prediction of collateral arteriole pathways appear successful in identifying "collateral" pathways in the microcirculation upon arteriole occlusion, the complexity of the networks and spread of hemodynamic changes apparent in our network-wide analysis suggest that there were a number of similarly recruited pathways missed by our simple prediction rules. However, even the argument that the observed differences in hemodynamic alterations were related to our ability to predict collateral routes between the networks of the two strains further highlights the necessity for examining hemodynamics across an entire network on an individual arteriole level. To date, the vast majority of studies examining the mechanism of collateral arterial development have focused on networks where it is easy to select for specific vascular segments that undergo reliable hemodynamic changes and/or growth such as the mesentery $(238,239)$, hindlimb ligation $(3,155)$, and pial circulation (68). In the microcirculation, the arteriovenous networks are often highly interconnected and variable between individual animals, making examinations of the coordination of hemodynamics and cell signaling mechanisms as related to microvascular remodeling challenging. Further, the interindividual variations necessitate the use of models that can chronicle these variables over time. Laser speckle flowmetry, therefore, presents a particularly compelling and powerful tool by being able to measure hemodynamics on an individual vessel basis, non-invasively, repeatedly, and without the need of exogenous contrast agents. As such, network-wide LSF can function as 
a means of stratifying hemodynamic changes across an entire microvascular network that can then be mapped back to the vascular remodeling or cell signaling/recruitment data obtained by other intravital microscopy techniques (Figure 4.6). Such combinations can pull from the strength of various intravital microcirculation preparations such as the ear $(217,240,241)$ which requires minimal surgical intervention or the dorsal skinfold window chamber for understanding of tumor vascularization (242) and vascular remodeling in response to injury/inflammation (90). While the scope of the current study does not cover how network alterations in hemodynamics guide network structural remodeling, the results demonstrate that laser speckle microscope has the potential to explore these questions in conjunction with existing intravital microscopy techniques to begin teasing apart the interplay of hemodynamics in short-term and long-term autoregulation. For example, laser speckle flowmetry could be used to measure the propagation of hemodynamic alterations through a network in response to a localized metabolic or electrical stimulus in order to quantify the relative contribution of circulating and vascular mural connection components to the conducted response of short-term network vasoactivity. Further, the non-invasive nature of laser speckle flowmetry could be combined with transgenic mice with fluorescent markers to understand the temporal recruitment of various immune cell populations driven by hemodynamic changes that guide long-term structural network remodeling.. Taken together, network-wide LSF could be particularly valuable in efficiently acquiring numerous datasets that capture velocity distributions throughout a network during short-term and longterm autoregulation to test and validate some of the autoregulatory insights gained from theoretical modeling (201).

\section{Limitations}

Several limitations are necessary to keep in mind when applying LSF to microvascular intravital imaging. First, though our data suggest a potentially broad relationship between speckle flow and absolute flow, this has not been verified in other preparations and the exact 
relationship may vary if using a different intravital arrangement. Therefore, the measurements from laser speckle are limited to quantifying the relative changes in hemodynamics in individual segments or a distribution throughout a network unless the individual intravital system has been calibrated. Second, there are numerous algorithms for converting raw speckle images to relative velocity. The one chosen in this study is a simplified version to improve processing time and is valid for the longer exposure times required for microcirculation preparations chosen here (206), but it may not be applicable to higher velocities seen in other intravital preparations. Lastly, the network-wide approach generates large quantities of data, which can be difficult to process. However, combinations of manual and semi-automated analysis tools, as in the current study, or automated analysis $(215,217)$ can be used to aid the process.

\section{Perspectives}

Here we demonstrated the use of laser speckle flowmetry as a quantitative tool for measuring relative blood velocity and shear stress in large microvascular networks at high resolution. The technique can be easily adapted to a variety of intravital microscopy preparations for examining the microcirculation without the need of exogenous contrast agent. Combining the laser speckle flowmetry technique with the wide array of other methods available to intravital preparations, it will be readily possible to directly examine how changes in hemodynamics coordinate long-term vascular remodeling in microvascular networks and how short-term vasoactivity can modulate network hemodynamics.

\section{Acknowledgements}


The authors would like to thank Ji Song for her expertise in cremaster muscle preparation. This work was supported by grants from the National Institutes of Health (R01 HL074082 and R21 HL098632 RJP) and the American Heart Association (10GRNT3490001, RJP). JKM was supported by a pre-doctoral fellowship from the American Heart Association (09PRE2060385) and National Institutes of Health Training grant (T32-HL007284). 


\section{Chapter 4 Figures}

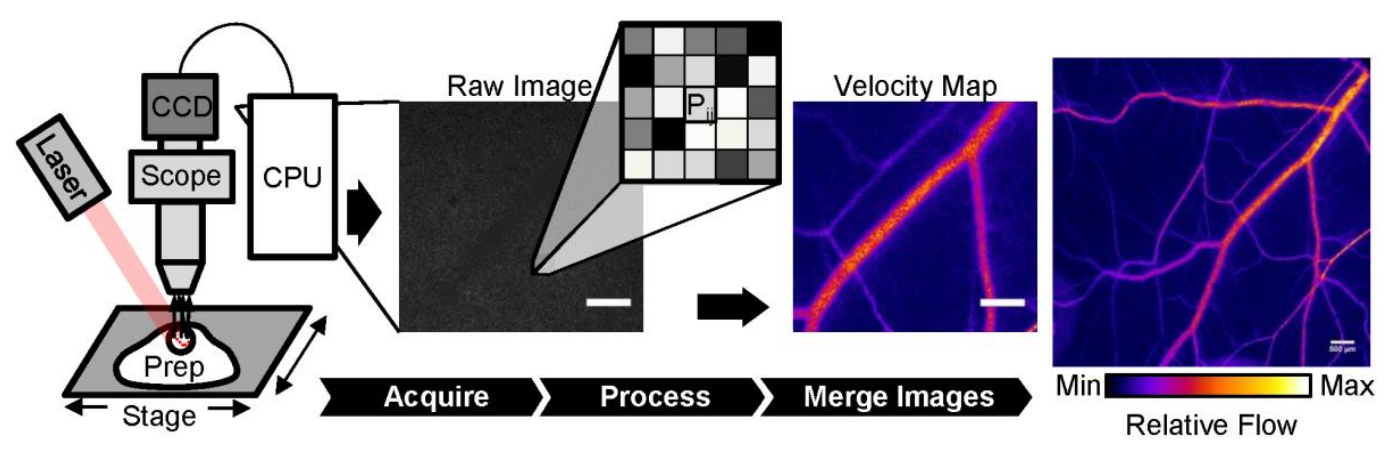

\section{Figure 4.1}

Generation of 2 dimensional blood velocity maps in large microvascular networks. A uniform field of laser light is used to illuminate the intravital tissue preparation. This produces a dynamic speckle pattern that is acquired by a CCD (or CMOS) camera. From the raw digital speckle image, a relative velocity measurement for each pixel $(\mathrm{P} i j)$, based on the variance of the speckle pattern in the surrounding $7 \times 7$ pixel neighborhood (Eq 1), is used to generate a relative velocity map at the given field of view. For more robust measurements, individual processed speckle images are averaged and then merged into the larger microvascular network (scale bar is $500 \mu \mathrm{m}$ for all images). 

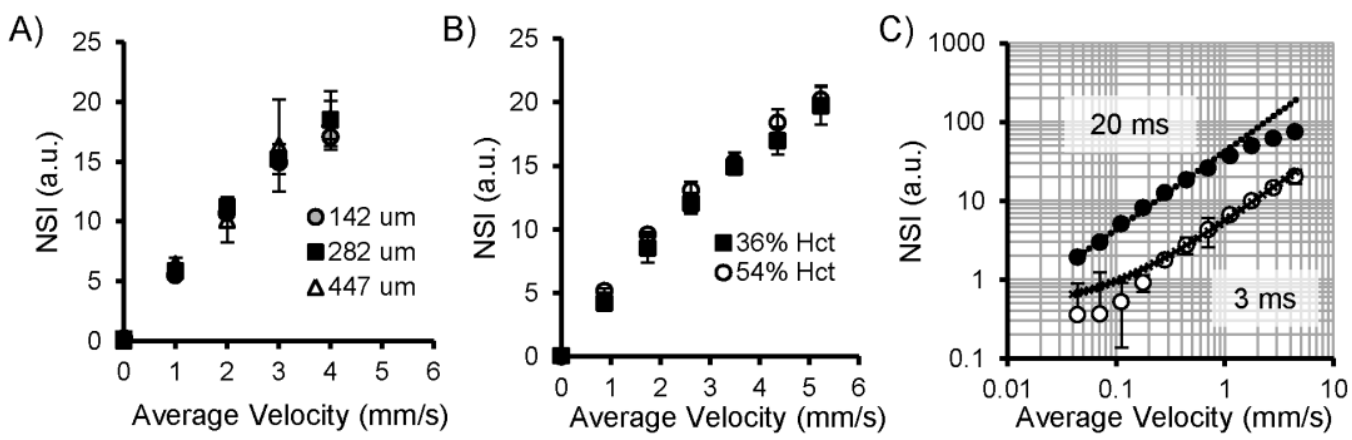

\section{Figure 4.2}

In vitro relationship between normalized speckle index (NSI, arbitrary units, a.u.) and absolute average velocity. A) Glass capillary diameter was increased to approximately 2- $(282 \mu \mathrm{m})$ and 3$(447 \mu \mathrm{m})$ fold of the initial $142 \mu \mathrm{m}$ diameter with no significant variation in the NSI-velocity relationship (3ms exposure). B) Increasing hematocrit from 36\% PCV to 54\% PCV demonstrated no significant variation in the NSI-velocity relationship (3ms exposure). C) The linear dynamic range (in log-log scale) of the in vitro NSI-velocity relationship can be shifted by adjusting exposure rate from $3 \mathrm{~ms}$ exposure (open circles) to $20 \mathrm{~ms}$ exposure (closed circles). Linear relationship (log-transformed) is displayed with dotted lines $(20 \mathrm{~ms}$, circles, excludes last 4 non-linear values; $3 \mathrm{~ms}$, cross-hatches, excludes first 4 non-linear values). 
Microsphere Velocimetry

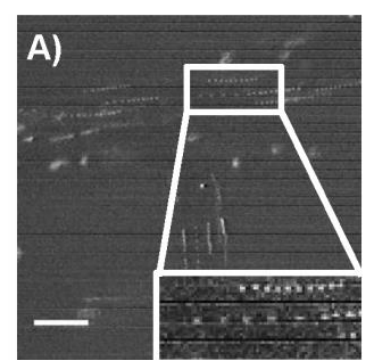

LSF Velocity Map

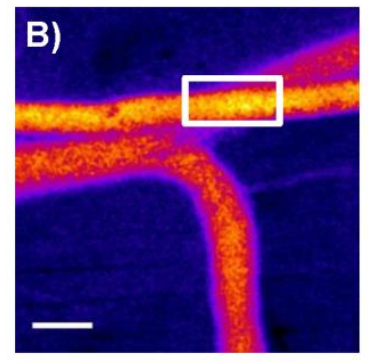

Min
Cremaster Muscle

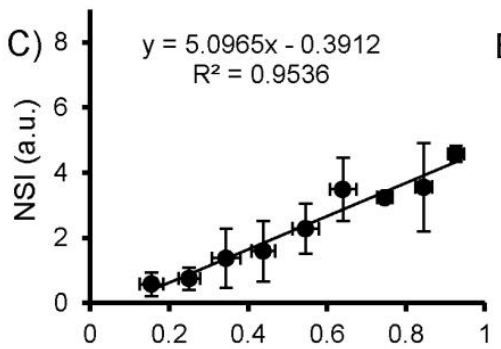

Microsphere Velocity $(\mathrm{mm} / \mathrm{s})$

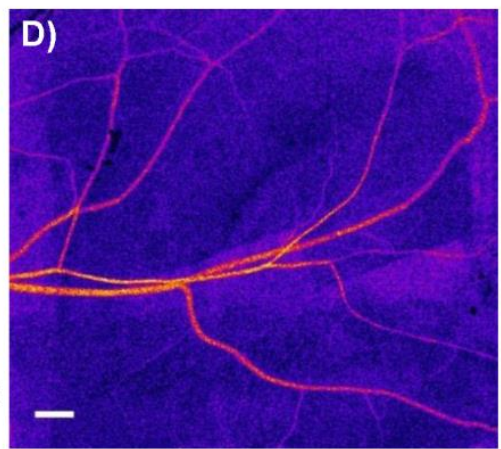

Dorsal Skinfold Window Chamber

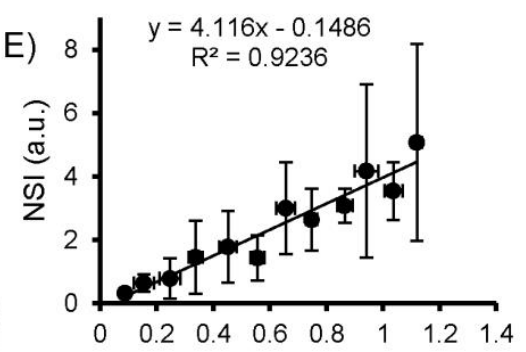

Microsphere Velocity $(\mathrm{mm} / \mathrm{s})$

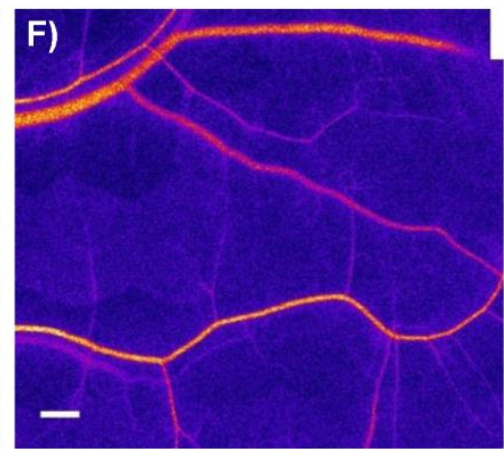

\section{Figure 4.3}

In vivo validation of NSI versus absolute velocity relationship. A) Circulating fluorescent microspheres were used to calculate centerline velocity (pulsed excitation source, 10ms intervals) at each field of view. Inset depicts microsphere traveling in centerline. B) LSF velocity maps were generated for the same field of view immediately after microsphere imaging (scale bar is $100 \mu \mathrm{m}$, 3ms exposure). This method was applied in mouse cremaster muscle and dorsal skinfold window chamber. $\mathrm{C}, \mathrm{E})$ In vivo relationships between NSI and centerline velocity in the mouse cremaster ( $C$, binned by $0.1 \mathrm{~mm} / \mathrm{s}$ divisions, $\mathrm{n}=4$ muscles) and dorsal skinfold ( $\mathrm{E}$, binned by $0.1 \mathrm{~mm} / \mathrm{s}$ divisions, $\mathrm{n}=5$ windows) microcirculation. D,F) Individual fields of view were then merged to form velocity distribution maps of entire microvascular networks ( $3 \mathrm{~ms}$ exposure, scale bar is $500 \mu \mathrm{m}$ ). 


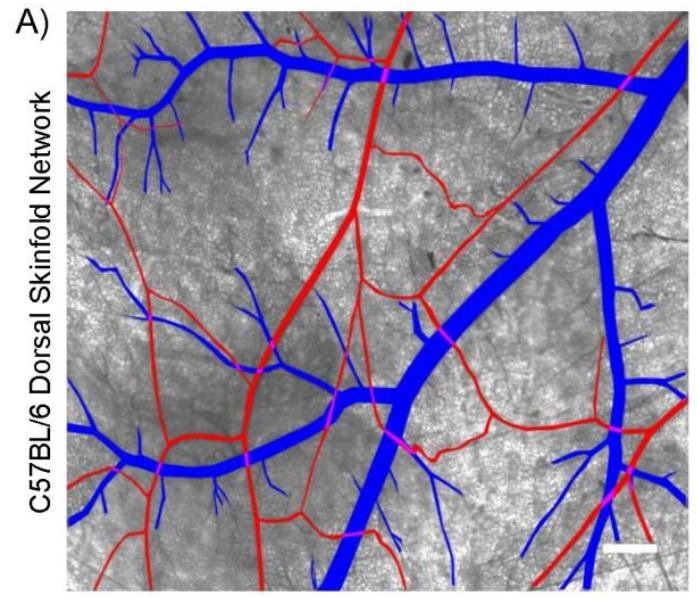

C)

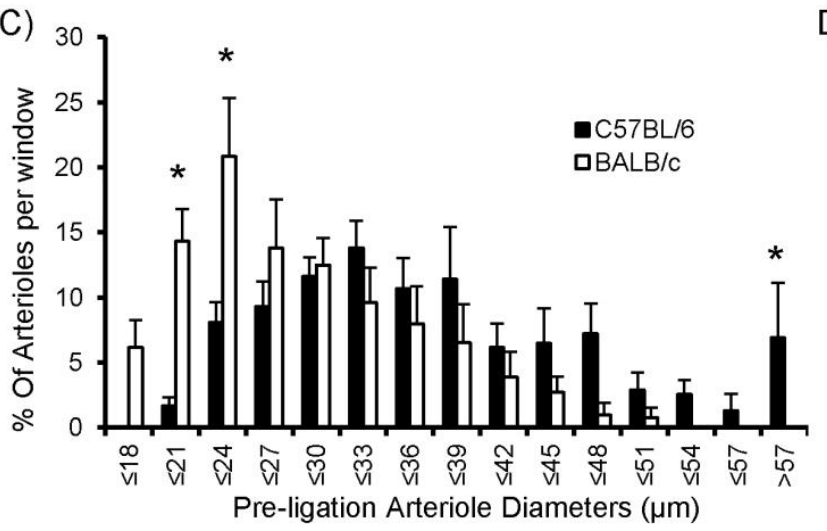

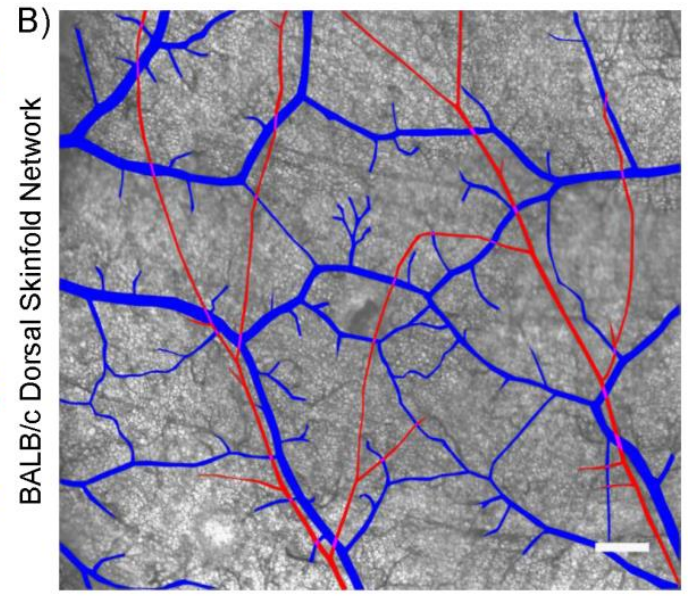

D)

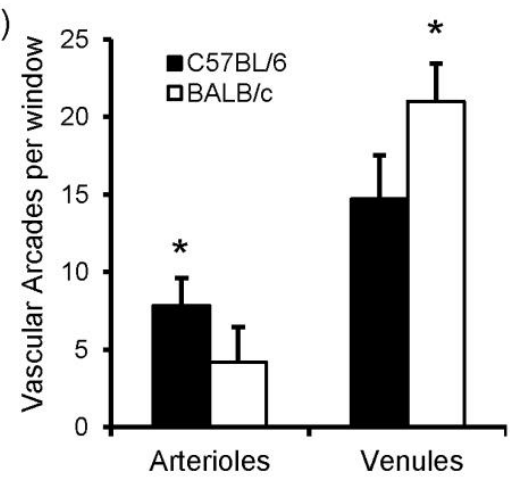

\section{Figure 4.4}

Dorsal skinfold window chamber network structure for C57BL/6 and BALB/c mouse strains. A, B) Demarcated brightfield images of dorsal skinfold window chamber microvascular networks demonstrate differences in network structure between $\mathrm{C} 57 \mathrm{BL} / 6$ and BALB/c strains (arterioles in red, venules in blue, scale bar is $500 \mu \mathrm{m})$. C) Histogram of pre-ligation arteriole diameters shows skewing of BALB/c segments toward smaller diameters and a significant increase in segments $>57 \mu \mathrm{m}$ in diameter in C57BL/ 6 networks ( ${ }^{*}$ indicates significantly different than other strain within same diameter bin at $p<0.05, n=5$ windows per strain). D) Microvascular arcade loop analysis demonstrates significantly more arteriole loops in C57BL/6 mice, but fewer venular loops compared to BALB/c networks ( ${ }^{*}$ indicates significantly different than other strain at $p<0.05, n=5$ windows per strain). 

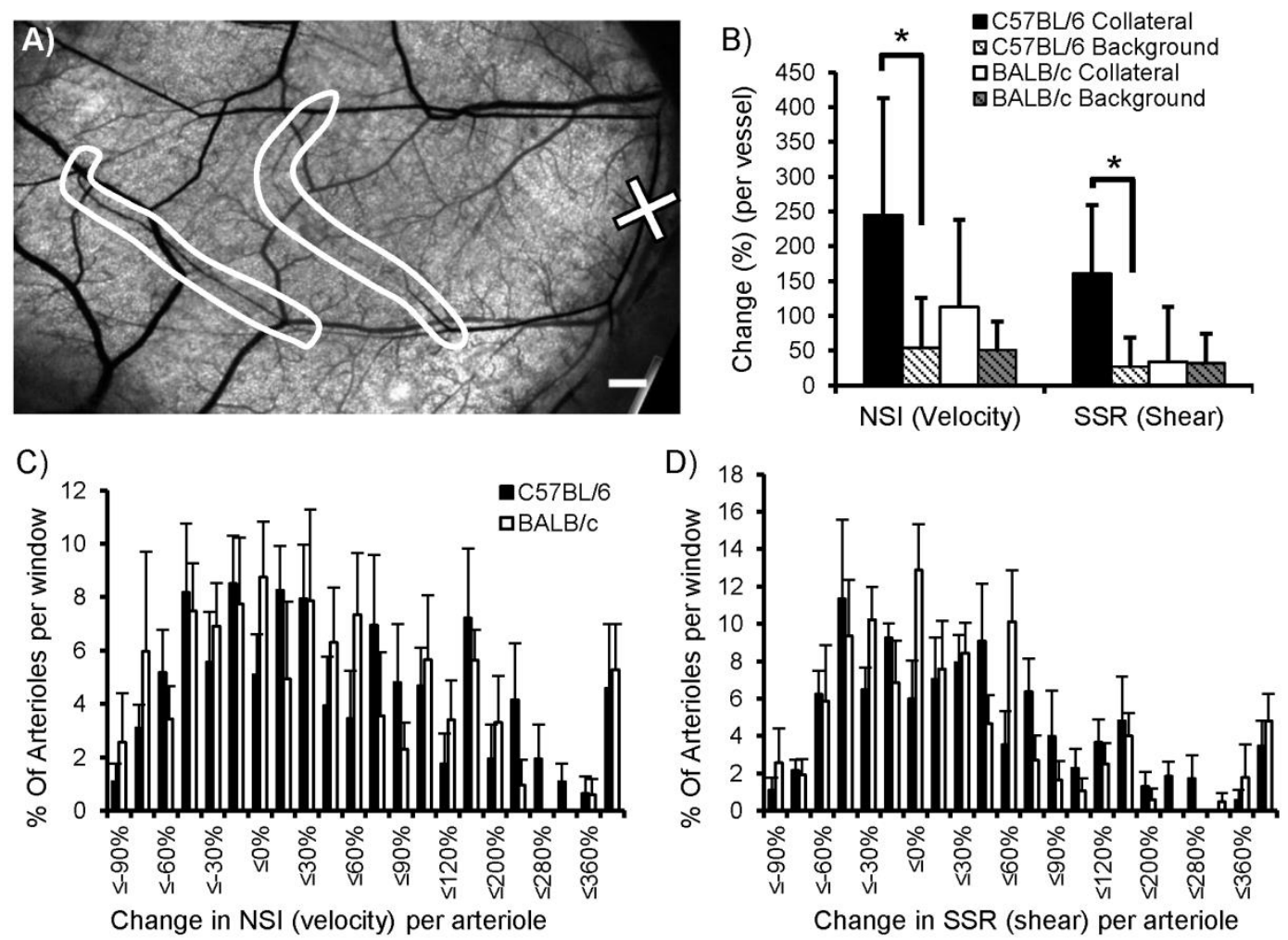

\section{Figure 4.5}

Network hemodynamic alterations after arteriole occlusion in C57BL/6 and BALB/C dorsal skinfold window chambers. A) Representative window chamber network with the chosen site for micro-occlusion (marked by ' $X$ ') and the predicted collateral pathways (circled in white) (scale bar is $500 \mu \mathrm{m}$ ). B) Bar graph analysis of collateral and background arteriole pathways shows significant increase in collateral velocity and shear stress from pre- to $24 \mathrm{hrs}$ post-microvessel occlusion for the C57BL/6 networks ( ${ }^{*}$, $p<0.05$ between collateral and background changes within strain, $n=5$ windows per strain). C, D) Histograms representing global analysis of hemodynamic changes (NSI, proportional to velocity, and SSR, proportional to wall shear stress) without stratification. No significant differences were observed between strains (binned by percent change, $n=5$ windows per strain). 


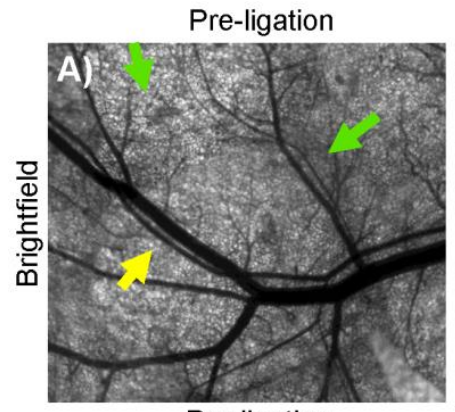

Pre-ligation

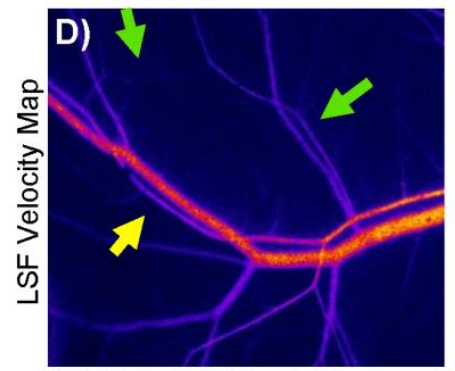

NSI (Velocity) (Pre $\rightarrow 24 \mathrm{hrs}$ post) SSR (Shear) (Pre $\rightarrow 24 \mathrm{hrs}$ post)

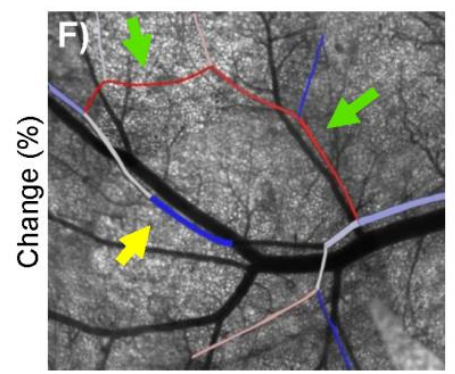

24hrs Post-ligation

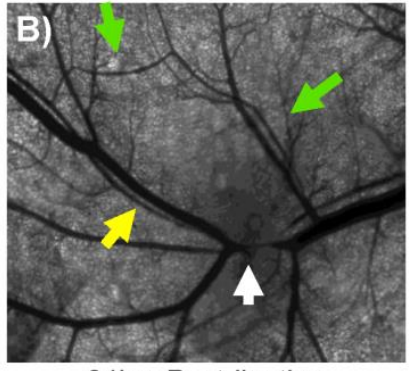

24hrs Post-ligation
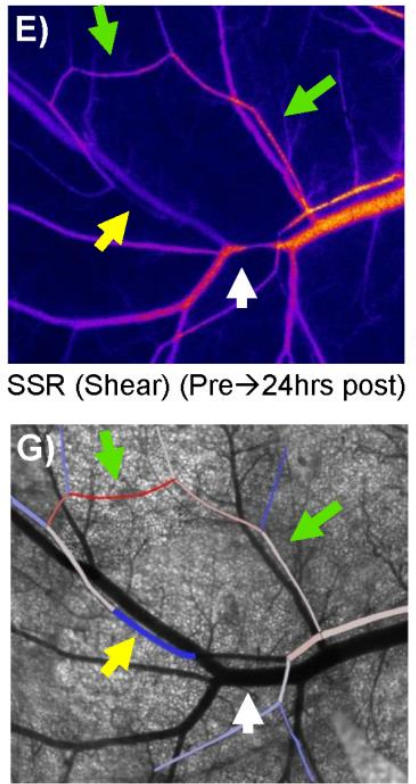

Day 6 Post-ligation

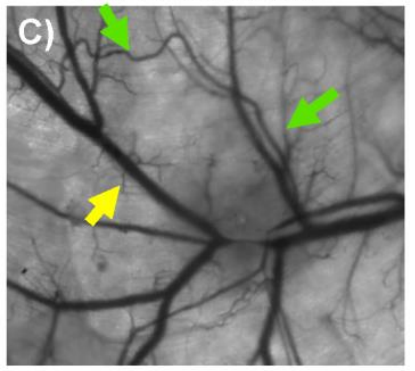

Speckle Flow (a.u.)
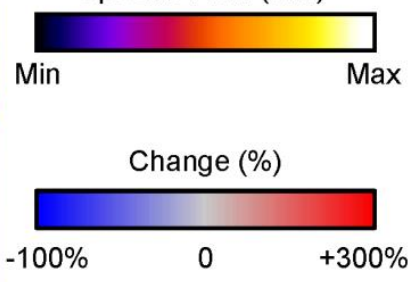

Diameter (24hrs $\rightarrow 6$ days post)

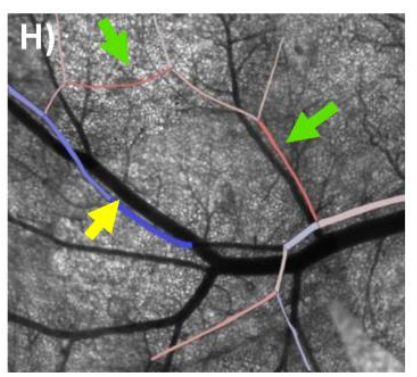

\section{Figure 4.6}

Illustration of how hemodynamic changes and microvascular structure may be mapped in individual microvessels over time using LSF. A-C) Brightfield images of network structure over 6 days after arteriole occlusion in an example window chamber network (green arrows denote collateral pathway exposed to increased velocity, yellow arrow denotes segment exposed to decreased velocity, white arrow indicates site of arteriole occlusion, C57BL/6 strain). Note the increased tortuosity, indicative of outward remodeling, along the high flow pathway. D, E) LSF velocity maps from pre- to $24 \mathrm{hrs}$ post-arteriole occlusion. F, G, H) Overlays of changes in velocity, shear stress, and diameter, respectively, on the preligation network demonstrate how changes in hemodynamics using LSF can be mapped back to changes in network structure in a preparation that allows for chronic measurements. 
Chapter 5: Trans-illuminated Laser Speckle Imaging of Deep Collaterals in Ischemic Mouse Hindlimb Reveals that Flow Direction Reversal is an Independent Arteriogenic Stimulus

Joshua K. Meisner, Joshua L. Heuslein, Jacqueline Niu, Xuanyue Li, Suna Sumer, Song J, Elizabeth G. Ames, Richard J. Price

(in preparation) 


\section{Introduction}

The importance of adequate remodeling of pre-existing arterial interconnections to form endogenous collateral bypasses - i.e. arteriogenesis - is highlighted in the extensive link between adequate collateral development and enhanced patient outcomes with arterial occlusive disease $(3,6,243,244)$. However, of the numerous clinical trials designed to therapeutically stimulate collateral development, few have proven successful. This is likely related to the fact that these interventions do not sufficiently recapitulate the complex sequence of processes that must be coordinated to achieve functional collateral development $(2,6,151)$. These initial failures have led to a re-examination of the basic mechanisms underlying collateral remodeling in the hope of identifying central signaling pathways for better therapeutic development.

The key initiating stimulus for arteriogenesis is altered shear stress along collateral arterial pathways (2). Upon occlusion of a major artery, downstream pressure is reduced, causing an increase in pressure gradient, blood flow, and shear stress along pre-existing collateral arteries that bypass the occlusion. Broadly speaking, it is known that the magnitude (245) and duration $(24,25)$ of increased shear stress determine maximal collateral outgrowth and eventual resolution. However, the topological arrangement of collateral arteries in skeletal muscle dictates that changes in shear stress from baseline after the occlusion of a major artery will be non-uniform along any given collateral pathway. For example, in a mouse model of moderate hindlimb ischemia (Figure 5.1A), some pre-existing collateral artery segments will experience a reversal of flow direction. We hypothesize that such changes in flow direction could have a profound influence on arteriogenesis, such as has been clearly demonstrated for reciprocating flow and recirculation zones in atherosclerosis (246). Nonetheless, quantitative measurements of these "segment-to-segment" variations in shear stress magnitude and direction along individual collateral arteries have not been reported. 
We believe this paucity of hemodynamic information is due, at least in part, to the lack of a method capable of making such measurements. The primary challenges are the small caliber of the developing collateral vessels (often $<100$ um in initial diameter (3)) and the need to integrate these data over a large area. Of the technologies with sufficient spatial resolution, such as multiphoton excitation fluorescence $(230,234)$, optical coherence tomography $(224,242)$, and photoacoustic tomography $(233,247)$, there are still multiple barriers to their adoption for studying arteriogenesis. These include cost and technical expertise, depth of imaging, and data integration over the observed vascular networks. One potential solution is laser speckle flow (LSF) microscopy, which we have recently shown to be a low-cost, efficient solution for quantifying change in hemodynamics across large microvascular networks (248). The use of LSF, however, has been limited by the depth of signal acquisition to the most superficial vascular structures (205), limiting its application for analysis of collateral arteries embedded deeply in the tissue. To increase acquisition depth, a recent report (249) suggested that reorientation of the illumination source to transmission through the tissue could project and capture the hemodynamic signals from deeper vascular structures.

Here, we developed a novel transillumination based LSF system to quantify, for the first time, the in vivo spatial distribution of collateral artery hemodynamics before and after femoral artery ligation in the mouse ischemic hindlimb, which is the most widely used model of peripheral arteriogenesis. Using these data in conjunction with a spatial assessment of collateral artery development, we show that flow direction change is an independent arteriogenic stimulus. In addition, collateral artery endothelial cells and those in culture are able integrate the directional reversal stimulus induced by femoral artery ligation into reorientation of planar cell polarization on the scale of hours. To then probe the mechanism(s) of enhanced arteriogenesis and sensitivity to direction stimulus in the flow-reversed collateral segment, we performed genome-wide transcriptional profiling and pathways analysis on cultured endothelial cells that 
had been exposed to flow-reversal conditions mimicking those measured in vivo through LSF. We found that the inclusion of the reversal of shear stress direction more fully activated many of the known pathways and transcription factors involved in arteriogenesis, including ERK 1/2 MAPK cascades $(25,250,251)$, PKA signaling $(252,253)$, NFKB $(173,254)$, and KLF2 (255). These data suggest that, in addition to shear stress magnitude increases from baseline, reversed shear stress direction should be considered in studies aimed at addressing mechanisms of hemodynamic arteriogenesis regulation.

\section{Materials and Methods}

An expanded section detailing the transillumination LSF method and techniques used to assess arteriogenesis, angiogenesis, planar cell polarization, and genome-wide transcriptional profiling through DNA microarrays can be found online at http://circres.ahajournals.org. Briefly, femoral artery ligations (FAL) on wild-type C57BL/6 mice were performed as previously described $(99,112,154,155)$ to produce consistent remodeling in the gracilis adductor muscle and the predicted hemodynamic stimuli depicted in Figure 5.1A. LSF-derived measurements of velocity and shear stress were calculated for transillumination and epi-illumination configurations (see Online Figure 5.I) using previously described algorithms $(206,248)$. Perfusion fixation $(4 \%$ paraformaldehyde) and vascular casting (MicroFil $\Theta$, FlowTech Inc, Carver, MA) were used to obtain regional measurements of lumenal diameter in the gracilis collateral network and to enable cross sectional analysis of remodeling and angiogenesis in specific regions (see Online Figure 5.II). Perfusion fixation (4\% paraformaldehyde) and whole mount immunofluorescence were used to obtain adluminal collateral artery diameter (anti-smooth muscle $\alpha$-actin) and planar cell polarization (anti-giantin or anti-pericentrin, for golgi apparatus or microtubule organizing center perinuclear localization, respectively) from specified collateral artery regions. Relative shear stress changes obtained from in vivo transillumination LSF measurements were used to simulate "FAL" conditions in human umbilical vein endothelial cell (HUVEC) cultures (see Figure 
5.5A). 6 hours after simulated "FAL", total RNA was isolated and genome-wide transcriptional profiling was performed using DNA microarrays (Affymetrix Human Gene ST 1.0 oligo microarrays), and analyzed using the Robust Multiarray Average (RMA) algorithm (256). Expression dataset is publically available at GEO (http://www.ncbi.nlm.nih.gov/geo/ (GSE 46248). All other statistical analyses were performed as one- or two-way ANOVA tests using the Holm-Sidak method for multiple comparisons. All results are reported as mean \pm standard error of the mean.

\section{Supplemental Methods}

All animal protocols were approved by the Institutional Animal Care and Use Committee at the University of Virginia and conformed to all regulations for animal use outlined in the American Heart Association Guidelines for the Use of Animals in Research. C57BL/6 mice were purchased from Charles River Laboratory (Wilmington, MA) and housed in the animal facilities at the University of Virginia.

\section{Femoral Arterial Ligation Model}

To produce uniform hemodynamic changes in the collateral arteries in a superficial adductor muscle, the femoral artery ligation scheme shown in Figure 5.1A was chosen. This particular ligation pattern has been previously shown to produce consistent arteriogenesis in the collateral arteries of the gracilis adductor muscles $(99,112,155,257)$ with minimal heterogeneity in the baseline collateral structure and with the predicted changes in flow direction from baseline.

Male mice, 9-13 weeks of age, were anesthetized (i.p $120 \mathrm{mg} / \mathrm{kg}$ ketamine, $12 \mathrm{mg} / \mathrm{kg}$ xylazine, and $0.08 \mathrm{mg} / \mathrm{kg}$ atropine), depilated, and prepped for aseptic surgery. On the left leg, an incision was made directly above and along the femoral artery, which was gently dissected from the femoral vein and nerve between the bifurcation of the superior epigastric artery and 
popliteal artery. Two 6.0 silk sutures were placed immediately distal to the epigastric artery, which served as the origin of the muscular branch artery in all mice, and the artery segment between the two ligatures was then severed with micro dissecting scissors. The surgical site was then closed with 6.0 prolene suture. A sham surgery, wherein the femoral artery was exposed but not ligated, was performed on the right hindlimb (i.e. on the other leg). Animals received one injection of buprenorphine for analgesia at the time of surgery and a second dose 8-12 hours later.

\section{Laser Doppler Perfusion Imaging}

For monitoring blood flow recovery and post-surgical ischemia, mice were anesthetized (i.p $120 \mathrm{mg} / \mathrm{kg}$ ketamine, $12 \mathrm{mg} / \mathrm{kg}$ xylazine, and $0.08 \mathrm{mg} / \mathrm{kg}$ atropine) then placed prone on a surgical heating pad for 5 minutes to minimize temperature variation. The soles of the feet were scanned (Lisca PIM laser Doppler imager), and mean voltage of foot perfusion was used to calculate relative perfusion ratio (ligated/unligated).

\section{Laser Speckle Flowmetry Setup and Data Analysis}

When a coherent light source passes through a static tissue, it creates a random interference pattern known as speckle. The speckle pattern produced is characteristic for a given tissue structure. When particles move within the given tissue (i.e. red blood cells), it causes the speckle pattern to fluctuate, such that when these fluctuations are integrated over time it is possible to use spatial statistics to quantify tissue motion (203). We have previously shown that using a simplified algorithm developed to remove the assumption of Gaussian or Lorentzian distributions (206), it is possible to quantify relative change in blood flow in the microcirculation at an individual vessel level (248). The approach related the change in speckle contrast (C, Eq. 1) to autocorrelation time (т, Eq. 2), which is inversely proportional to blood velocity (SI, speckle intensity, Eq. 2). 


$$
C_{i j}^{2}=\frac{\sigma_{i j}^{7 x 7^{2}}}{\left\langle I_{i j}^{7 x 7}\right\rangle^{2}}(1) \quad \frac{1}{\tau}=\frac{1}{2 T C^{2}}=S I(2)
$$

Here $C$ is derived on a per pixel basis from the standard deviation ( $\sigma$, Eq. 1) and mean pixel intensity (〈I〉, Eq. 1) values in a $7 \times 7$ neighborhood. For our calculations, we used a simplified algorithm (Eq. 2) for relating speckle contrast $(C)$ and exposure time ( $T$ ) to autocorrelation time ( $\tau$, Eq. 2). Vessel speckle velocity was normalized (NSI) to the background tissue according to Eq. 3. As previously described (248), this process removes background artifact from general tissue motion and variations in speckle illumination to allow for quantitative comparison of velocity change across experiments.

$N S I=\frac{S I_{\text {vessel }}}{S I_{\text {background }}}-1$

In our arrangement, a 30mW, 658nm laser diode (LPM658-30, Newport Corporation, Irvine, CA) was coupled to a fiber optic cable and used to illuminate the mouse hindlimb or tissue phantom (Online Figure 5.I) from either an epi-illumination position or trans-illumination orientation, where the fiber output was placed at the base of the (phantom) tissue. For both the in vitro and in vivo testing, a cooled, monochrome CCD camera (Optronics Quantifier, Goleta, CA) $(7.4 \mu \mathrm{m} \times 7.4 \mu \mathrm{m}$ pixels) was used to acquire the raw speckle images through an intravital microscope (Zeiss Axioskop,Thornwood, NY) using a 4x air objective (Zeiss Acroplan LD $N A=0.1$ ). As previously described (248), the objective and camera were chosen to balance the resolution of the scope with the size of the speckle to satisfy the Nyquist sampling criteria of at least 2 pixels per individual speckle (220). For each field of view, laser position, and flow setting, a sequence of $\geq 15$ 12-bit raw speckle images were acquired with $5 \mathrm{~ms}$ exposure time to capture average velocity over multiple cardiac cycles. Camera gain and light path intensity were varied to maintain mean pixel intensity of the raw speckle image. To minimize the influence of whole background tissue motion from mouse movement, the processed speckle intensity (SI) images 
were normalized to median background intensity. Code for converting raw speckle images into speckle flow maps was developed, implemented, and analyzed in Fiji (156), and is freely available at request. Individual flow images were then merged into larger 2D maps using Adobe Photoshop (CS2, Adobe Systems Inc, San Jose, CA).

\section{In Vitro Speckle Tissue Phantom Imaging Protocol}

While initial studies suggested that speckle signal analysis from transmitted laser light yields dominant vascular signals from deeper tissue structures (249) and that speckle signals remain viable through up to $2 \mathrm{~cm}$ of biological tissue (258), the quantitative applicability of signal processing used for epi-illumination versus transillumination was unknown. Therefore, light scattering tissue phantoms were used to test speckle velocity linearity between transillumination and epi-illumination speckle arrangements. Phantoms were generated as previously described (248) by adding titanium dioxide nanopowder (677469, Sigma Aldrich, St Louis, MO) to 184 Sylgard PDMS gel (Dow Corning, Midland, MI) at $10 \mathrm{mg} / \mathrm{mL}$ and pouring the mixture over optical glass-bottomed molds. Phantoms were then allowed to cure overnight at $37^{\circ} \mathrm{C}$. Molds $0.5 \mathrm{~cm}$ thick were composed of polyethylene tubing connected to glass capillary tubes with $142 \mu \mathrm{m}$ inner diameter (Drummond Microcap, P1799 Sigma Aldrich, St Louis, MO) placed at 0, 120,360 , and $720 \mu \mathrm{m}$ from the surface.

For validating the linear operation of the speckle processing algorithm for both epiillumination and trans-illumination arrangements, a sequence of known flows of defibrinated bovine blood (Hemostat Labs, Dixon, CA) was driven via a syringe pump (PHD2000, Harvard Apparatus, Holliston, MA) through the phantom. The randomized sequence and image acquisition was controlled by a custom-designed LabVIEW program (National Instruments, Austin, TX)_available at request. Only data from the most superficial capillary tube was 
analyzed. Each complete program of flow rates was repeated 5 times for both epi-illumination and trans-illumination configurations.

For testing the effect of increasing depth on speckle signal attenuation, a single flow rate was applied to the phantom and raw speckle images were captured at each capillary tube depth $(0,120,360$, and $720 \mu \mathrm{m})$. Image capture sequence at each depth setting was repeated 5 times for both epi-illumination and trans-illumination configurations.

\section{In vivo analysis in transillumination hindlimb}

For imaging of gracilis collateral hemodynamics, an intravital microscopy setup was used similar to that depicted in Online Figure 5.I. Mice were anesthetized (i.p $120 \mathrm{mg} / \mathrm{kg}$ ketamine, $12 \mathrm{mg} / \mathrm{kg}$ xylazine, and $0.08 \mathrm{mg} / \mathrm{kg}$ atropine), depilated, and prepped for aseptic surgery. On the left leg, a window of skin was dissected free and retracted directly above the superficial adductor muscles. The femoral artery was gently dissected from the femoral vein and nerve between the bifurcation of the superior epigastric artery and popliteal artery. One 6.0 silk suture was loosely placed immediately distal to the epigastric artery, which served as the origin of the muscular branch artery in all mice. During surgical preparation, the mouse body temperature was maintained by a surgical heating pad and the exposed tissue was moistened with warmed Ringer's physiological saline solution (RPSS, $137.9 \mathrm{mM} \mathrm{NaCl}, 4.7 \mathrm{mM} \mathrm{KCl}, 1.2 \mathrm{mM}$

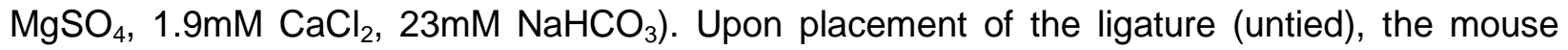
was transferred to the intravital microscopy station, and the exposed tissue was maintained under a constant drip of warmed RPSS that was deoxygenated with $5 \% \mathrm{CO}_{2}$ balance $\mathrm{N}_{2}$, and set to maintain $37^{\circ} \mathrm{C}$ fluid temperature. Mice were allowed 15 minutes to equilibrate under the drip conditions before baseline imaging. After the equilibration period, a baseline (pre-ligation) series of image acquisitions using epi-illumination and then trans-illumination laser orientation were taken at multiple fields of view to encompass the gracilis collateral arteries between the 
saphenous and muscular branch arteries (Figure 5.1B). Between each series of raw speckle images (epi- and trans-illumination), images of the collateral arteries were taken using brightfield epi-illumination through an objective-mounted fiber optic light guide to allow for lumenal diameter measurements (A08650, Schott Inc, Elmsford, NY). After baseline acquisition, the ligature was tightened, and stoppage of blood flow through the femoral artery was visually confirmed in all mice. After 30 minutes post-ligation, trans-illumination speckle and brightfield then epi-illumination and brightfield imaging sequences were repeated. Predicted baseline and post-ligation flow direction was confirmed in an identical preparation on a separate set of mice through fluorescent imaging of intravascularly injected fluorescent microspheres (FP-3070-2, Spherotech Inc, Forest Lake, IL).

For analyzing hemodynamic changes, the assumption of Poiseuille flow was made, where wall shear rate is proportional to average blood velocity divided by tube diameter. This assumption is valid for most cases in the microcirculation (200). Therefore, proportional wall shear rate (SSR, speckle shear stress) was calculated using the NSI measurement of average blood velocity and vessel diameter $(D)$ at each designated location (Eq 4).

$S S R \propto N S I / D(4)$

\section{Tissue Harvesting for Cross Sectional Analysis and Collateral Structure by Vascular Casting}

For analysis of lumenal diameters in the gracilis collateral arteries and to enable sectioning at specific regions, vascular casting was performed using an opaque polymer that allows for accurate lumenal diameter measurements (155). At specified time points (1, 3, and 7 days) after femoral artery ligation, mice were euthanized, and the abdominal aorta was cannulated. The lower body was then perfused with $2 \%$ heparinized saline with $2 \mathrm{mM}$ adenosine (16404, Fisher Scientific, Pittsburg, PA) and 0.1mM papaverine (P3510, Sigma Aldrich, St 
Louis, MO) to clear and vasodilate the downstream vasculature at a constant rate of $1 \mathrm{~mL} / \mathrm{min}$ (PHD2000, Harvard Apparatus) and then fixed with 4\% paraformaldehyde solution (19943, Affymetrix, Cleveland, $\mathrm{OH}$ ). The lower body was then perfused with $0.8 \mathrm{~mL}$ of the casting agent Microfil@ (FlowTech, Inc, Carver, Massachusetts) at a constant pressure of $100 \mathrm{mmHg}$. Viscosity of Microfil@ was adjusted to minimize transport across capillaries. After curing overnight at $4^{\circ} \mathrm{C}$, gracilis muscles were dissected free and then cleared in $50 \%$ glycerol in phosphate buffered saline (PBS) overnight. Cleared tissues were mounted between two coverslips using $500 \mu \mathrm{m}$ thick spacers (645501, Grace Bio-Labs Inc) to keep constant thickness between muscles. Muscles were imaged using transmitted light at 4x magnification on a Nikon TE200 inverted microscope with a CCD camera (Quantifier, Optronics Inc). Individual fields of view were montaged together (Photoshop CS2, Adobe Systems Inc). After imaging, muscles were rehydrated, cut, and then paraffin embedded for cross sectional analysis at the muscular branch and saphenous artery entrance regions to the collateral arteries made visible by vascular casting (Online Figure 5.II).

\section{Tissue Harvesting for Collateral Structure by Immunofluorescence}

For analysis of adluminal diameter in the gracilis collateral artery network, smooth muscle alpha-actin immunofluorescence was performed on whole mount gracilis muscles. At specified time points $(7,14$, and 28 days) after femoral artery ligation, mice were anesthetized, and the exposed gracilis collaterals were vasodilated by drip perfusion of $10^{-4} \mathrm{M}$ adenosine in RPSS. Mice were then euthanized, and perfusion fixed via cardiac cannulation through a series of $2 \%$ heparinzed saline and $10 \%$ neutral buffered formalin at a constant rate of $1 \mathrm{~mL} / \mathrm{min}$. After 30 minutes of fixation, gracilis muscles were harvested, washed, and then incubated for 48 hours at $4^{\circ} \mathrm{C}$ in 1:200 anti-smooth muscle alpha actin - Cy3 (1A4 clone, C6198, Sigma Aldrich, St Louis, MO) in antibody and blocking buffer (PBS with $0.1 \%$ saponin, $2 \%$ BSA, and $0.2 \%$ sodium azide). Muscles were then washed and cleared in $50 \%$ glycerol in PBS overnight at $4^{\circ} \mathrm{C}$. 
Clear tissues were mounted between two coverslips using $500 \mu \mathrm{m}$ thick spacers $(645501$, Grace Bio-Labs Inc). Muscles were imaged using transmitted light at 4x magnification on a Nikon TE200 microscope with a CCD camera (Quantifier, Optronics Inc). Individual fields of view were montaged (Photoshop CS2, Adobe Systems Inc).

\section{Tissue Harvesting for In Vivo Collateral Artery Endothelial Planar Cell Polarization}

For analysis of the spatial variation of endothelial cell planar polarization in the gracilis collateral arteries, whole gracilis muscles were immunolabeled for smooth muscle alpha-actin, the golgi apparatus, and the nucleus. The golgi apparatus was chosen over the more widely reported microtubule organizing center (MTOC) (259-261) due to its larger size in order to aid identification in the situation of decreased resolution and clarity when imaging thick $(>100 \mu m)$ tissues using confocal microscopy. The golgi apparatus, which colocalizes with the MTOC (262), has previously been shown to serve as a functional marker of endothelial cell planar polarization (263). Pre-ligation and 24 hours after femoral artery ligation, mice were euthanized, and the abdominal aorta was cannulated. The lower body was then perfused with $2 \%$ heparinized saline with $2 \mathrm{mM}$ adenosine and $0.1 \mathrm{mM}$ papaverine to clear and vasodilate the downstream vasculature at a constant rate of $1 \mathrm{~mL} / \mathrm{min}$ (PHD2000, Harvard Apparatus) and then fixed with $2 \%$ paraformaldehyde solution for 30 minutes. Tissues were then flushed with PBS and perfused with an antibody and blocking solution consisting of PBS, $2 \%$ BSA, $10 \%$ NGS, and $0.015 \%$ saponin and containing anti-smooth muscle $\alpha$-actin-FITC (1:400 1A4 clone, A5691,

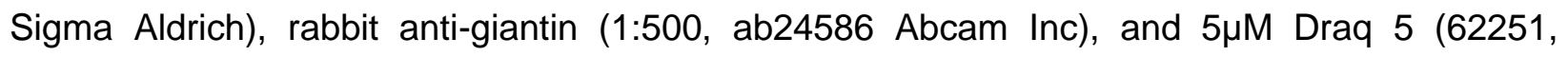
Thermo Scientific Inc). This perfusion step was found necessary to ensure adequate penetration of the giantin antibody to the collateral artery endothelial cells. After 60 minutes, muscles were removed and further incubated overnight at $4^{\circ} \mathrm{C}$ in an antibody solution consisting of PBS, $2 \%$ BSA, 10\% NGS, and 1\% TritonX100 and containing anti-smooth muscle alpha actin-FITC

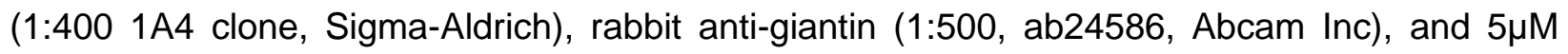


Draq 5. Muscles were then washed and incubated overnight at $4^{\circ} \mathrm{C}$ in a secondary antibody solution consisting of PBS, $2 \%$ BSA, and $1 \%$ TritonX100 and containing goat anti-FITC Alexa 488 (1:200 A11090, Life Technologies Inc), goat anti-rabbit IgG Alexa 555 (1:200, A21428 Life Technologies Inc), and $5 \mu \mathrm{M}$ Draq 5 . Muscles were then washed and cleared in a series of $50 \%$ glycerol in PBS and $90 \%$ glycerol in PBS overnight at $4^{\circ} \mathrm{C}$. Cleared muscles were then mounted between two coverslips using $500 \mu \mathrm{m}$ thick spacers (645501, Grace Bio-Labs Inc). Muscles were then imaged on a Nikon TE2000 C1 laser-scanning confocal microscope with a 20x oil lens immediately upstream of the first visible transverse arteriole branch off of the anterior and posterior collateral arteries extending from the muscular branch and saphenous arteries and at the midpoint of the anterior and posterior collateral arteries.

\section{Cross Sectional Analysis for Regional Capillary Density and Muscle Fiber Atrophy}

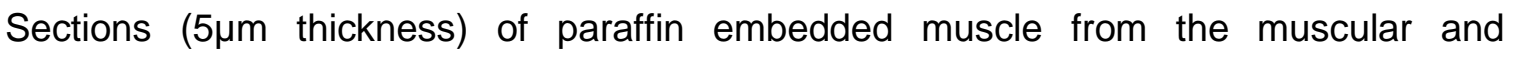
saphenous regions (Online Figure 5.II) were rehydrated and labeled with Griffonia simplicifolia isolectin- $B_{4}$ conjugated to Alexa 647 (GSL-I-B $10 \mu \mathrm{g} / \mathrm{ml}$, I32450 Life Technologies Inc) and 10nM SYTOX green nuclear stain (S7020, Life Technologies Inc). The entire cross-section for each region (encompassing both the anterior and posterior heads of the gracilis muscle) was imaged at 20x magnification on a Nikon TE2000 C1 confocal microscope and individual fields of view were stitched together (Photoshop CS2, Adobe Systems Inc). All GSL-I-B 4 positive vessels $<7 \mu \mathrm{m}$ in diameter and all muscle fibers (identified by autofluorescence) were counted in each section using Fiji (156). Total muscle area was determined by semi-automated threshold analysis for calculation of average muscle fiber area. 


\section{Regional Analysis of Collateral Artery Structure and Hemodynamics}

For analysis of lumenal and medial diameter from intact gracilis collateral whole mounts (i.e. vascular casting and smooth muscle alpha-actin immunofluorescence), collateral entrance regions were defined according to the following method (Online Figure 5.II). A cropped portion $(560 \mu \mathrm{m} \times 560 \mu \mathrm{m})$ of the montaged image (previously randomized and de-identified) was taken of the collateral artery at the first visible branch point of a terminal arteriole from the primary collateral as it extended from either the saphenous or muscular branch artery. This was done for each primary collateral running through the anterior and posterior heads of the gracilis muscle, yielding 4 total image regions per tissue whole mount. After each cropped image region was taken, all images were randomized and de-identified. The mean diameter was then taken from three separate diameter measurements along the length of cropped portion of the collateral artery. An identical procedure was performed for the processed montages obtained from laser speckle imaging with trans-illuminated laser orientation and the paired brightfield montage to enable calculation of relative velocity (NSI, Eq 3), diameter (D, Eq 4), and shear rate (SSR, Eq 4).

\section{Human Umbilical Vein Endothelial Cell Culture}

Human umbilical vein endothelial cells (HUVECs) purchased from Cell Applications Inc., VEC Technologies Inc. (Rensselaer, NY), and Lonza were unthawed and maintained on $0.1 \%$ gelatin coated flasks in M-199 medium (Lonza), supplemented with 10\% fetal bovine serum (Life Technologies Inc, Grand Island, NY), 100U/mL penicillin-G $+100 \mathrm{ug} / \mathrm{ml}$ streptomyocin (Life Technologies Inc) , 2mmol/L L-glutamine (Life Technologies Inc), 5ug/ml endothelial cell growth supplement (Biomedical Technologies, Stoughton, MA), and 10ug/ml heparin (Sigma Aldrich Inc). For each set of experimental comparisons, cells were used from the same company and cell line, in their third subculture passage. 


\section{In vitro Exposure of Endothelial Cells to Simulated Ligation Shear Stress}

HUVECs were plated on cell culture grade plastic dishes coated with $0.1 \%$ gelatin and grown to confluence. A cone and plate flow apparatus (264), which maintains cells at $5 \% \mathrm{CO}_{2}$ and $37^{\circ} \mathrm{C}$, was used to induce a shear stress protocol. The applied shear stress protocol consisted of a 24 hour preconditioning period at a steady of 15 dyne $/ \mathrm{cm}^{2}$ and then either increased to 30 dynes $/ \mathrm{cm}^{2}$ (non-reversed), increased to 30 dynes $/ \mathrm{cm}^{2}$ and reversed in direction (reversed), or held constant at 15 dynes $/ \mathrm{cm}^{2}$ (control) (Figure 5.5A). Fresh culture media consisting of M199 with 4\% dextran from Leuconostoc spp (Sigma Aldrich, $\mathrm{M}_{\mathrm{r}} \sim 500,000$ ), $2 \%$ fetal bovine serum, $100 \mathrm{U} / \mathrm{mL}$ penicillin-G $+100 \mathrm{ug} / \mathrm{ml}$ streptomyocin, $2 \mathrm{mmol} / \mathrm{L} \quad$ L-glutamine, $5 \mathrm{ug} / \mathrm{ml}$ endothelial cell growth supplement, and $10 \mathrm{ug} / \mathrm{ml}$ heparin was added to cells before exposure to shear stress and was continuously exchanged throughout the duration in the cone and plate apparatus.

\section{HUVEC RNA isolation, qRT-PCR, and Microarray Gene Expression Profiling}

For RT-PCR and cDNA microarray assays, total RNA was extracted using the PureLink total RNA purification system (Life Technologies Inc). RNA concentration and purity was confirmed with a NanoDrop spectrophotometer for RT-PCR and by Agilent Bioanalyzer. Each replicate consisted of three plates split from the same culture. The three plates were then run in tandem, with one plate run under each of the three separate shear conditions.

For microarray analysis, 4 replicates were collected for each condition at 6 hours postsimulated "FAL". RNA amplification, labeling, hybridization with Human Gene 1.0ST oligo microarrays (Affymetrix), quality control, and raw data acquisition were performed by the University of Virginia Biomolecular Research facility and data analysis by the University of Virginia Bioinformatics Core facility. All preprocessing and analysis was done using $\mathrm{R}$ version 2.15.0. CEL files were imported using the affy package, version 1.34.0. Expression intensities 
were summarized, normalized, and transformed using Robust Multiarray Average (RMA) algorithm (256). Probesets were annotated using the bioconductor annotation package hugene10sttranscriptcluster.db version 8.0.1, using the annotate package, version 1.34.0. Probesets not mapping to an Entrez gene were excluded (probesets mapping to RIKEN genes were not excluded). For examining differential gene expression, a linear model with empiricalBayes moderated standard errors was fit using the limma package in $\mathrm{R}$. The BioConductor package arrayQualityMetrics was used to perform quality assessment on the preprocessed, summarized, normalized, transformed, filtered data. Heat maps were created using the gplots package in R (http://CRAN.R-project.org/package=gplots). Gene ontology was assessed through the gene ontology enrichment analysis and visualization tool (GOrilla) (265). Biological context was analyzed by Ingenuity Pathway Analysis (http://www.ingenuity.com/) for functional gene clustering and function.

For quantitative reverse transcriptase PCR (qRT-PCR), total RNA was reverse transcribed using the iScript cNDA synthesis kit (Bio-Rad, Hercules, CA). RT-PCR was performed on CCL2 (forward 5'-CCAGCAGCAAGTGTCCCAAAG -3' reverse 5'TGCTTGTCCAGGTGGTCCATG-3'), eNOS (forward 5'-CTCCATTAAGAGGAGCGGCTC-3', reverse 5'-CTAAGCTGGTAGGTGCCTGTG-3'), E-selectin (forward 5'AATCCCAGTTTGTGAAGCTTTCCA-3', reverse 5'-GCCAGAAGCACTAGGAAGACAATT-3'), ICAM (forward 5'-TCGCTATGGCTCCCAGCAGC-3', reverse 5'TTCCGGTTGTTCCCAGGCAGG-3'), KLF2 (forward 5'-GCTGAGTGAACCCATCCTGCC-3', reverse 5'-CGCTGTTGAGGTCGTCGTCG-3'), KLF4 (forward 5'GGCCAGAATTGGACCCGGTGTAC-3', reverse 5'-CTGCCTTTGCTGACGCTGATGA-3'), and VCAM (forward 5'-GTTTGTCAGGCTAAGTTACATATTGATGA-3' reverse 5'GGGCAACATTGACATAAAGTGTTT-3'), with SYBR Green (Roche Applied Sciences) on a CFX96 Real Time Detection System (Biorad). Expression was normalized to $\beta 2$-microglobulin 
(forward

5'-AGCATTCGGGCCGAGATGTCT-3',

reverse

5'-

CTGCTGGATGACGTGAGTAAACCT-3') which is endogenously expressed and is not altered by many stimuli including shear stress(266). Normalized expression was quantified using the comparative $2^{\Delta \Delta \mathrm{Ct}}$ method.

\section{Immunofluorescence Labeling for HUVEC Planar Cell Polarity}

After exposure to our shear stress protocol, cells were fixed in 4\% PFA for 10 minutes at room temperature, blocked with PBS $+0.1 \%$ saponin $+2 \%$ BSA $+5 \%$ normal goat serum for 45 minutes at room temperature, and then incubated in primary antibody solution containing

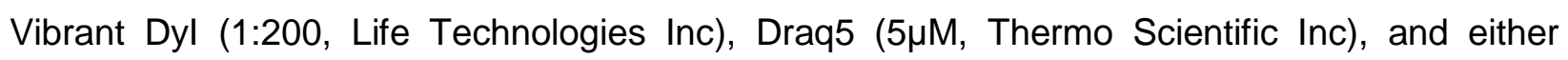
rabbit-anti-giantin (1:1000, ab24586, Abcam Inc) or rabbit-anti-percentrin (1:500, ab4448, Abcam Inc). Cells were washed three times in PBS $+0.1 \%$ saponin then incubated in goat-antirabbit Alexafluor 488 (1:500, Life Technologies) secondary antibody solution for 45 minutes. Cells were washed twice in PBS, then twice in 50:50 PBS:glycerol, and then in 10:90 PBS:glycerol prior to cover slip mounting with Prolong Gold (Life Technologies). Plates were allowed to cure overnight then were imaged using a Nikon TE2000 C1 laser scanning confocal microscope. The relative location of the nucleus to the Golgi apparatus or MTOC from three independent sets of experimental conditions, each an average of three representative fields of view (for a total of nine images per experimental condition), was determined by a blinded, independent observer.

\section{Data Analysis of Endothelial Cell Planar Polarity}

For analysis of endothelial orientation with respect to flow, maximum intensity projections of collateral arteries (for whole mount analysis) or fields of view (for HUVEC plates) were indicated with flow direction then de-identified for blinded analysis. Peri-nuclear location of 
the Golgi apparatus or MTOC was determined with respect to flow direction and nuclear position (Figure 5.4B).

\section{Statistical Analysis}

All results are reported as mean \pm standard error. All data were first tested for normality. Statistical significance was assessed by one- and two-way ANOVA, followed by paired comparisons using the Holm-Sidak method for multiple comparisons (SigmaStat 3.5, Systat Inc). Significance was assessed at $\mathrm{P}<0.05$.

\section{Results}

\section{Transillumination Laser Speckle Flow Microscopy Decreases the Signal Attenuation from Increasing Depth In Vitro}

We used an in vitro tissue phantom to test whether the linearity of the relationship between normalized speckle index (NSI) and velocity that was originally derived for epiillumination remains valid for trans-illumination LSF (Figure 5.2A). As predicted, both epiillumination and trans-illumination produced a similar and highly linear relationship between the speckle velocity measurement, $\mathrm{NSI}$, and known velocity (Figure $5.2 \mathrm{~A}$ ). To test the relative sensitivity of trans-illumination and epi-illumination to speckle velocity signal from blood vessels of increasing depth, tissue phantoms were created with artificial vessels placed at multiple depths. The trans-illumination configuration showed reduced sensitivity to increasing tissue depth with an approximate $50 \%$ reduction of the exponential decay of the speckle velocity signal with increasing depth compared to epi-illumination $\left(1.20 \cdot 10^{-3}\right.$ versus $2.70 \cdot 10^{-3}$, Figure $5.2 \mathrm{~B}$ at a constant velocity of $5 \mathrm{~mm} / \mathrm{sec}$ ). As such, trans-illumination maintained $40 \%$ of the initial superficial signal out to $720 \mu \mathrm{m}$ deep within the tissue phantom, versus $15 \%$ with epiillumination. 


\section{Transillumination LSF Microscopy Demonstrates Regional Variation of Hemodynamic Changes Along Collateral Arteries After Femoral Artery Ligation.}

A femoral arterial ligation (FAL) model, as depicted in Figure 5.1A, was used to induce arteriogenesis in the gracilis adductor muscle. In agreement with previous studies $(112,155)$, perfusion measurements of the distal tissue in the plantar surface of the foot indicate a moderate level of ischemia with full reperfusion back to baseline perfusion ratios by day 7 postFAL (Online Figure 5.III). Further, the chosen ligation scheme was predicted to yield a spatial variation in blood flow direction and magnitude along the length of the collateral arteries (Figure 5.1A) from the feeding muscular branch artery (also known as the lateral caudal femoral artery) to re-entry into the downstream vascular tree at the saphenous artery. The predicted flow directions were confirmed by observation of circulating fluorescent microspheres during intravital imaging, indicating reversal of initial blood flow direction at the saphenous artery entrance to the gracilis collateral network (data not shown, $n=4$ mice).

LSF microscopy was used to quantify relative velocity changes along the gracilis collateral network prior to FAL and 30 minutes post FAL (Figure 5.1B). Consistent with the in vitro findings, the trans-illumination configuration preserved the hemodynamic signal of the collateral artery network embedded within the gracilis muscle to a greater degree than the epiillumination configuration (Online Figure 5.IV). Because there were no changes in depth of the collateral artery network within the gracilis muscle from pre- to 30 minutes post-FAL, normalized speckle index could be used to quantify relative velocity change at the muscular branch and saphenous artery entrance regions (Figure 5.1C). When coupled with diameter measurements through brightfield imaging, the velocity changes were used to calculate relative change in wall shear rate (Eq. 4) from pre- to 30 minutes post-FAL (Figure 5.1D). As expected, there was an increase in blood flow and velocity along the gracilis collateral network. Interestingly, the percent increase in velocity $(102.9 \pm 9.5 \%$ and $99.7 \pm 11.9 \%$ for $\mathrm{MB}$ and SA entrance regions, 
respectively, $p=0.84)$ and shear stress $(102 \pm 12 \%$ and $97 \pm 10 \%$ for MB and SA entrance regions, respectively, $\mathrm{p}=0.73$ ) were not different at the muscular branch and saphenous artery entrance regions (Figure 5.1C, 5.1D, data from $n=10$ mice). The central region of the gracilis collateral network showed the largest relative change in velocity after FAL, going from negligible flow (minimal signal prevented accurate baseline measurement) pre-FAL to high velocity postFAL (Figure 5.1B). The switch from convergent blood flow toward the central region of the gracilis muscle pre-FAL from both the saphenous and muscular branch arteries to high flow across the length of the collateral artery was present in all mice, matching the predicted hemodynamic patterns seen in Figure 5.1A. Quantitative measurements of diameter and, therefore, SSR were not obtained for the central region due to the greater depth of the collateral artery within the muscle tissue in the mid-zone, leading to decreased brightfield visualization.

\section{Gracilis Collateral Arteries Exhibit Increased Arteriogenesis in Flow Reversal Regions.}

To determine if the regional differences in hemodynamics relate to regional differences in collateral remodeling, we measured both lumenal (i.e. vascular casting, Figure 5.3A) and smooth muscle cell adluminal (i.e. immunofluorescence, Figure 5.3B) collateral diameter. Specifically, we focused our measurements on the muscular branch and saphenous artery entrance regions to isolate the influence of change in directionality. By both lumenal and adluminal measurements, the saphenous artery (reversing) entrance region experienced significantly greater lumenal expansion than the muscular branch artery (non-reversing) entrance (Figure 5.3C and 5.3D).

After 24 hours, neither region demonstrated lumenal expansion compared to the unligated control limb (ligated vs unligated: $45.4 \pm 3.4 \mu \mathrm{m}$ vs $39.6 \pm 2.9 \mu \mathrm{m}, \mathrm{p}=0.18$, muscular branch, $42.7 \pm 2.4 \mu \mathrm{m}$ vs $36.2 \pm 3.7 \mu \mathrm{m}, \mathrm{p}=0.14$, saphenous). By day 3 , however, both regions 
had undergone significant lumenal expansion that had begun to diverge between the reversing (saphenous, $67.8 \pm 4.0 \mu \mathrm{m}$ vs $42.9 \pm 2.4 \mu \mathrm{m}, \mathrm{p}<0.001$, ligated vs unligated) and non-reversing (muscular branch, $55.5 \pm 1.6 \mu \mathrm{m}$ vs $46.7 \pm 2.4 \mu \mathrm{m}, \mathrm{p}=0.04$, ligated vs unligated, $\mathrm{p}=0.004$ muscular versus saphenous within ligated) regions. Outward remodeling and divergence between reversing and non-reversing regions increased further by day 7 post-ligation (93.7 \pm $9.1 \mu \mathrm{m}$ vs $38.6 \pm 2.1 \mu \mathrm{m}, \mathrm{p}<0.001$, saphenous, $70.7 \pm 6.1 \mu \mathrm{m}$ vs $48.5 \pm 3.6 \mu \mathrm{m}, \mathrm{p}=0.01$, ligated vs unligated; muscular branch; $p=0.01$ muscular versus saphenous within ligated) (Figure 5.3D). At day 7 post-FAL, the extent of remodeling within the convergent (central) region was in between reversing (saphenous) and non-reversing (muscular branch) entrance regions such that only the reversing and non-reversing entrance regions showed significant divergence (ligated: $70.7 \pm 6.1 \mu \mathrm{m}, 84.7 \pm 8.9 \mu \mathrm{m}, 93.7 \pm 9.1 \mu \mathrm{m}$, muscular, central, saphenous, respectively; $p=0.11$ central vs muscular, $p=0.30$ central vs saphenous, $p=0.01$ saphenous versus muscular) (unligated: $48.5 \pm 3.6 \mu \mathrm{m}, 39.0 \pm 2.8 \mu \mathrm{m}, 38.6 \pm 2.1 \mu \mathrm{m}$, muscular, central, saphenous, respectively; all p>0.25) (Figure 5.3C, data not shown for central region). After day 7, the increased outward remodeling of the reversing and non-reversing regions stabilized with no further increases in adluminal diameter (Figure 5.3D). The greater degree of outward remodeling in the reversing entrance region was maintained out to 28 days post-ligation. These regional differences in the fully developed day 28 collateral artery represent a $51 \%$ greater increase in collateral diameter within the reversing region $(81.3 \pm 9.9 \%$ and $132.5 \pm 2.1 \%$ versus their unligated controls, saphenous and muscular branch entrances, respectively, $n=9)$. The time course of remodeling matches well with the laser Doppler reperfusion measurements (Online Figure 5.III), where resting foot perfusion returns to baseline around 5 days postligation, with no further increases in reperfusion.

Cross-sectional analysis of the reversing and non-reversing entrance regions was used to confirm arteriogenic wall mass increases. Both reversing and non-reversing regions showed 
significant hypertrophy and outward remodeling in terms of increased wall thickness, wall area, and inner diameter versus their respective unligated control arteries at day 7 post-FAL (Online Figure 5.V). However, similar to the results from the whole mount analysis, there was a greater increase in wall area $\left(1112 \pm 177 \mu \mathrm{m}^{2}\right.$ vs $291 \pm 25 \mu \mathrm{m}^{2}, \mathrm{p}<0.01$ versus unligated, reversing; 752 $\pm 101 \mu \mathrm{m}^{2}$ vs $275 \pm 54 \mu \mathrm{m}^{2}, p<0.01$ versus unligated, nonreversing; $p<0.05$ reversing ligated vs non-reversing ligated) and diameter $(79.9 \pm 8.2 \mu \mathrm{m}$ vs $31.4 \pm 2.1 \mu \mathrm{m}, \mathrm{p}<0.01$ versus unligated, reversing; $58.4 \pm 6.3 \mu \mathrm{m}$ vs $29.4 \pm 4.4 \mu \mathrm{m}, \mathrm{p}<0.01$ versus unligated, nonreversing; $\mathrm{p}<0.01$ reversing ligated vs non-reversing ligated) at 7 days post-FAL in the cross sections from the reversing region compared to the cross sections from the non-reversing region (Online Figure 5.V).

\section{No Evidence of Hypoxia in Developing Collateral Regions}

To examine the potential role of ischemia in the spatial differences in arteriogenesis between the flow reversing and non-reversing regions, we performed cross sectional analysis to quantify functional evidence of tissue hypoxia. Similar to previous findings for the gracilis muscle in this FAL model (142), capillary to muscle fiber ratio (Online Figure 5.VI) showed no evidence of angiogenesis in either the muscular branch and saphenous artery regions $(1.64 \pm 0.09$ vs $1.52 \pm 0.10, p=0.39$ ligated vs unligated, saphenous (reversing); $1.67 \pm 0.08$ vs $1.41 \pm 0.11$, $\mathrm{p}=0.07$ ligated vs unligated, muscular branch (non-reversing)). As a second measure of tissue ischemia, muscle fiber size in the gracilis muscle (Online Figure 5.VI) showed no evidence of atrophy in either the muscular branch or saphenous artery regions $\left(719 \pm 21 \mu \mathrm{m}^{2}\right.$ vs $706 \pm 12$ $\mu \mathrm{m}^{2}, \mathrm{p}=0.10$ ligated vs unligated, saphenous (reversing); $697 \pm 37 \mu \mathrm{m}^{2}$ vs $742 \pm 34 \mu \mathrm{m}^{2}, \mathrm{p}=0.62$ ligated vs unligated, muscular branch (non-reversing)). 


\section{Endothelial Cell Planar Polarization Demonstrates Orientation and Reorientation to Predicted Flow Directions.}

To determine the degree of endothelial cell planar polarization (PCP) toward the direction of blood flow in the collateral arterioles of the gracilis muscle, we quantified the perinuclear position of the golgi apparatus with respect to predicted flow direction pre- and 24hrs post-FAL (Figure 5.4). The strength of directional polarization was quantified similar to that previously described for the microtubule organizing center (259) (Figure 5.4B). As predicted, at baseline, the endothelial cells at the collateral entrance regions had an upstream orientation to their respective feeding arteries (muscular branch and saphenous arteries) (Figure 5.4 and Online Figure 5.VII). The central region of the collateral artery showed no significant polarization toward either direction. By 24 hours post-FAL, however, both the central and saphenous entrance regions showed strong polarization toward (upstream direction) the muscular branch artery (Figure 5.4D), such that the degree of PCP toward the new flow direction within the saphenous artery entrance region was similar to that pre-FAL.

\section{HUVECs Exhibit Planar Polarization and Directional Responsiveness to Simulated Femoral Artery Ligation}

To isolate the influence of directional change on endothelial cell responsiveness to the hemodynamic changes induced by FAL, the changes in shear stress and direction quantified by trans-illumination LSF measurements were applied to human umbilical vein endothelial cell (HUVEC) cultures (Figure 5.5). A value of $15 \mathrm{dynes} / \mathrm{cm}^{2}$ shear stress was chosen as a baseline shear stress, within normal physiological range (246). Preconditioning for $24 \mathrm{hrs}$ at the baseline shear stress was used to establish endothelial cell PCP toward the pre-conditioned flow direction to mimic the in vivo baseline state. FAL was then simulated by a step-wise $100 \%$ increase in shear stress in the same direction or in the opposite direction (Figure 5.5A) to match 
the changes in shear stress experienced in the muscular branch and saphenous artery entrance regions, respectively. The control plates (maintained at 15 dynes $/ \mathrm{cm}^{2}$ ) showed an upstream polarization similar to that seen in vivo (Figure 5.4) at 2 and 6 hours post-step change. This upstream polarization was maintained (at 2 hours) or enhanced (at 6 hours) with a step-wise increase in shear stress in the same direction. However, when shear direction was reversed, endothelial cells transitioned from the PCP induced by preconditioning toward the new upstream direction. At 2 hours after reversal, HUVECs exhibited no significant PCP (Figure 5.5C). By 6 hours after reversal, HUVECs had achieved a degree of PCP similar to that of baseline, but toward the new direction (Figure 5.5C). While labeling of the golgi apparatus was used to aid visualization in the highly scattering gracilis muscle tissue, we confirmed the use of golgi perinuclear position matches well with the more widely used metric measurements of PCP via perinuclear position, as previously reported (263) (Online Figure 5.VIII). These data demonstrate sensitivity to directional change in shear stress in HUVECs similar to that seen in vivo, with complete re-orientation of PCP toward the new upstream direction within 6 hours after reversal.

\section{Flow Direction Reversal Broadly Enhances the Arteriogenic Gene Expression Pattern Seen with Increased Shear Stress}

To explore how differential endothelial cell signaling induced by change in flow direction could lead to enhanced arteriogenesis, we performed genome-wide transcriptional analysis on HUVECs exposed to the relative changes in shear stress induced by femoral artery ligation as measured through trans-illumination LSF. The time-point of 6 hours post simulated FAL was chosen because PCP reorientation is complete and robust transcriptional changes in response to a stepwise shear stress increase have been reported at 6 hours (267). Using a false discovery rate for significance of $<0.10$ obtained through the Robust Multiarray Average (RMA) algorithm (256), HUVEC cultures undergoing a reversal of direction showed an approximate 10fold greater number of transcripts altered by the increase in shear stress (280 upregulated, 251 
downregulated, reversed versus control, RvC, Online Table I) compared to cultures without a change in direction (21 upregulated, 25 downregulated, non-reversed versus control, NvC, Online Table II) (Figure 5.6A). Interestingly, the genes altered by shear stress increase alone $(\mathrm{NvC})$ demonstrated a similar expression pattern as those seen with the addition of reversed direction, where there was $87 \%$ overlap with the significant RvC genes in the same expression direction (Figure 5.6B). Of the 537 genes with FDR $<0.1$ from both groups, only $2.6 \%$ of genes demonstrated regulation in opposing directions between reversed and non-reversed conditions (Figure 5.6C). Of those, all had fold change $<0.1$ and FDR $>0.88$ in the non-reversed group. While the additional mechanical stimulus of flow reversal induced a much larger degree of transcriptional change, the expression pattern was very similar to that seen in the non-reversed conditions, where $91.4 \%$ of all genes with FDR $<0.1$ were altered to a greater degree in the reversed condition than non-reversed condition (Figure 5.6B). With the changes of expression occurring in similar directions from the control state, there were no significant differences between reversal and non-reversal expression levels with an FDR $<0.20$. An a priori selection of 7 genes was used to validate the microarray results through real-time quantitative PCR (Table 1). All genes show similar expression patterns and significance between microarray and RTPCR measurements. However the increased sensitivity through RT-PCR demonstrated significant differences between reversal and non-reversal conditions within NOS3, ICAM1, and KLF2, which are known to play key roles in arteriogenesis $(33,112,255)$.

\section{Flow Reversal With Increased Shear Stress Magnitude More Fully Activates Key Arteriogenic Pathways When Compared to Increased Shear Stress Magnitude Alone}

Initial assessment of activated molecular functions was conducted through the gene ontology analysis (265) to assess over-representation of molecular pathways. The top 500 genes based on p-value ranking for increased shear stress, with and without flow reversal, as 
compared to control were used to understand the board functional processes involved in both conditions (Online Figure 5.IX, Online Table 5.III). There was only one significantly enriched molecular function in the case of the non-reversed increased shear stress: oxidoreductase activity. In the case of the additional reversed directional stimulus case, there was activation of multiple molecular functions including: reelin receptor activity, RNA pol II promoter transcription factors, cAMP phosphodiesterase activity, and GTPase regulation (Online Figure 5.IX, Online Table 5.III).

Additional function annotation, clustering, and analysis of predicted upstream regulators were performed by Ingenuity Pathways Analysis (http://www.ingenuity.com). Clustering of expression changes of all genes with FDR $<0.1$ along canonical pathways shows activation of several key pathways known to be involved in arteriogenesis, including cGMP signaling $(112,268)$, protein kinase A signaling $(252,253)$, MAPK signaling $(25,251,269)$, and cell-cell adherens junction signaling $(254,270,271)$ (Online Table IV). Reversed cultures showed much stronger activation of these canonical pathways versus the non-reversed cultures (Online Table IV). When the molecules within these canonical pathways from both conditions were grouped into phosphodiesterase, protein kinase A (PKA), MAPK, and cell-cell junction signaling, each of these clusters showed stronger activation under the reversed direction condition compared to the non-reversed condition (Figure 5.6). By further analyzing the transcription patterns for their potential upstream regulators, Ingenuity Pathways Analysis identified many of the known signaling pathways involved in arteriogenesis including growth factors (e.g. VEGF $(272,273)$, HGF (274,275), TGF $\beta$ (276), FGF2 (277,278), MAPK signaling $(25,251,269)$, PI3K signaling (11), and activation of the NFKB pathway $(173,279,280)$. Further, these pathways represent some of the critical proposed elements to endothelial responsiveness to altered shear stress when going from static culture to laminar shear stress (e.g. NFkB activation $(254,281)$ and MAPK signaling (282)). Of particular interest is that the strongest predicted upstream regulator 
is VEGF. Such predicted activation may not indicate autocrine signaling via secreted VEGF, but may be more indicative of VEGFR2 activation through a complex with VE-cadherin and PECAM1, which has been shown to be a central mechanosensory component to the application of shear stress $(254,283)$ and arteriogenesis $(271)$. Across all of these signaling pathways and upstream regulators, however, a clear activation is only apparent under the reversed direction condition. These data suggest that the shear stress-sensitive mechanotransduction pathways in endothelial cells, which are necessary for arteriogenesis, are more fully activated when there is a switch in the direction of blood flow.

\section{Discussion}

We report here the technical development and application of a novel laser speckle flowmetry (LSF) method for measuring blood velocity in collateral arteries in response to FAL in the widely used mouse ischemic hindlimb model (Figures 5.1 and 5.2 and Online Figures 5.1 and 5.IV). Using this new technology, we provide the first ever measurements of velocity and shear stress changes that occur in developing collateral arteries with respect to both longitudinal position and flow-direction, which reverses with FAL in well-defined regions (Figure 5.1). Our ability to make these hemodynamic measurements with high spatial resolution along the collateral arteries provided several new insights into the hemodynamic regulation of arteriogenesis. Foremost, we determined that collateral artery segments that are exposed to both an increase in shear stress magnitude and a reversal of flow direction exhibit markedly amplified arteriogenesis when compared to collateral artery segments exposed to increased shear stress magnitude alone (Figure 5.3 and online Figure 5.V). Endothelial cells in collateral segments exhibiting amplified arteriogenesis repolarized to the new upstream direction within 24 hours (Figures 5.4 and online Figure 5.VII), confirming that flow-direction had reversed and offering the hypothesis that signaling pathways associated with repolarization could be linked to amplified arteriogenesis. Meanwhile, capillary density in muscle tissue surrounding the flow- 
reversed collateral segments was unchanged from control (Online Figure 5.VI), indicating that amplified arteriogenesis was hypoxia-independent. Applying the LSF-derived "reversed-flow with increased shear stress magnitude" collateral artery shear stress pattern to cultured ECs also yielded EC repolarization (Figure 5.5), consistent with in-vivo observations. Genome-wide transcriptional analysis of these ECs revealed that flow-reversal with increased shear stress magnitude yielded an $\sim 10$-fold increase in significantly regulated transcripts over ECs exposed to increased shear-stress alone (Figure 5.6). This includes a set of potent arteriogenesis regulators (NOS3, ICAM-1, and KLF-2) that were then confirmed by RT-PCR (Table 1). Furthermore, IPA analysis indicated that flow-reversal more fully activates a number of important canonical arteriogenesis pathways and upstream regulators (Figure 5.6, Table 5.2). Going forward, the ability to capture these shear stress changes in vivo using our adapted LSF technology and to link markedly amplified collateral arteriogenesis to a set of signaling pathways that are more fully activated by flow-reversal may have important bearing on identifying novel targets for therapeutic revascularization strategies toward PAD.

\section{Application of Transillumination LSF for Generating Novel Measurements of In Vivo Hemodynamics in Small Arterial Networks}

Despite the known importance of hemodynamic stimuli in driving collateral development, there is a surprising lack of quantitative data on the hemodynamic changes within these arteries due to their small size $(<100 \mu \mathrm{m}(3))$ and variation in which collateral pathways develop across surgical models (3). In the current study, we chose to use a moderate ischemia model that produces a consistent and characteristic pattern of hemodynamic changes in the gracilis adductor muscle. By adapting the LSF technique to incorporate a transillumination configuration, we dramatically reduced the attenuation of the speckle signal with increasing depth, which has previously limited the usefulness of the technique to very select instances, without impacting the relationship for quantifying relative velocity (Figure 5.2). Therefore, we 
were able to harness the power of intravital LSF microscopy to generate the first quantitative measurements of altered shear stress and velocity in individual collateral arterioles. Because the software and hardware components necessary for the technique are widely available and present a minimal financial barrier for integration into existing intravital imaging setups, this adapted technique could rapidly expand to additional models and provide similarly difficult to obtain hemodynamic data at the individual microvessel level.

\section{Spatial Measurements of Altered Hemodynamics and Growth Identify Flow Reversal as a Potential Enhancer of Arteriogenesis}

From the in vivo shear stress data obtained from our adapted LSF method, we can demonstrate that the physical forces along collateral arteries that are critical for inducing arteriogenesis $(19,25)$ are not uniform. By measuring velocity changes across collateral arteries, there appear to be at least three distinct hemodynamic conditions: a non-reversing increase in shear stress near the feeding entrance to the collateral loop, an increase in shear stress from low/oscillating flow to sustained high shear stress at the central anastomotic region, and an increase in shear stress but in a reversed direction at the downstream outlet back into the occluded arterial tree. Interestingly, this latter region, with flow reversal, demonstrated the greatest degree of arteriogenesis. Considering that the central region went from near zero to high shear stress - the greatest relative increase between the three regions-but still did not achieve the same degree of arteriogenesis as the reversed region, we further argue that the influence of a complete reversal of shear stress direction leads to greater arteriogenesis versus an even larger relative change in shear stress. Therefore, we chose to focus on the two entrance regions to isolate the influence of flow reversal alone, without differences in the relative magnitude of an acute increase in shear stress. Importantly, the spatial variations observed here are not related to hypoxia and metabolic signaling as has been shown in a saphenous artery excision model $(284,285)$. The differential hypoxic environment across the muscle in the 
saphenous artery excision model can be seen in the increased capillary density within the saphenous entrance region (143) that is not present in our model (Online Figure 5.VI). Together

these data suggest flow reversal as a novel, independent stimulus for arteriogenesis. Additionally, they suggest that the location of tissue collection and analysis when studying arteriogenesis in hindlimb ischemia models needs to be chosen carefully, as each region may represent differential signaling cascades.

\section{Endothelial Cell Cytoskeletal Repolarization from a Pre-Conditioned Baseline As}

\section{A Potential Mechanism of Enhanced Arteriogenic Signaling}

A key motivation for the current study is that there is a paucity of data that have examined endothelial responsiveness to increased shear stress from a pre-conditioned baseline, which are the actual shear stress conditions that are believed to drive arteriogenesis in vivo. This is, in part, due to the lack of in vivo hemodynamic measurements available before and after femoral artery ligation. Of the handful of studies that consider baseline physiological conditioning, initial evidence suggested a $20 x$ reduction in the number of genes that are sensitive to step-wise increases in shear stress from a pre-conditioned baseline versus from static culture (86 versus $\sim 2000$ as reported by Zhang and Friedman (267)). Our data matches well with the closest similar study by Zhang and Friedman (267), who showed activation of 86 genes at FDR $<0.1$ at 6 hours after a non-reversed $100 \%$ increase in shear stress. While there was no overlap of the genes with an FDR $<0.1$ to those reported by Zhang et al, $73 \%$ of the genes were expressed in the same direction in the non-reversed condition at the same time point. For the reversal conditions, there was $20 \%$ overlap of genes at FDR $<0.1$ with $73 \%$ expressed in the same direction. More importantly, gene expression changes induced by the application of laminar shear stress from static culture are not wholly predictive of gene expression changes from an increase in shear stress of the same magnitude from a baseline shear stress (267). Additionally, those few studies that have incorporated non-reciprocating 
directional changes have shown that reversal of the direction of shear stress from baseline have a disproportionate impact on responses such as permeability (286), which is hallmark in the initiation of arteriogenesis (2). Even the application of very low (to -2.5 dynes $/ \mathrm{cm}^{2}$ ) levels of reversing shear stress elicits a disproportionately larger transcriptional response (of a similar pattern) than a greater increase in shear stress $\left(+10\right.$ dynes $\left./ \mathrm{cm}^{2}\right)(287)$. Together, these data suggest that the EC responsiveness to altered shear stress is dependent on its initial conditions, and the initial conditions present across a given collateral network need to be accounted for by incorporating baseline conditions when dissecting the mechanistic pathways of arteriogenesis.

Our results, additionally, raise the hypothesis that endothelial planar cell polarization may be linked to arteriogenic signaling pathways activated by the reversal of shear stress direction. Reorientation of PCP operates through small GTPases (i.e. GSK3 $\beta$, Rac1, RhoA and Cdc42 $(259,260,288,289))$ that are involved in arteriogenesis $(25,290)$ and were activated with flow reversal (Online Table III, online Figure 5.IX). The additional degree of cytoskeletal reorganization-most dramatically seen with the Golgi apparatus and MTOC repolarizationneeded to adapt to a $180^{\circ}$ change in shear direction, as compared to an increase in shear stress alone, may have increased arteriogenesis through coincident small GTPase signaling. Moreover, the additional step of transitioning through a depolarized state during reversal (Figure 5.5) could have prolonged this coincident signaling compared to that of the central region (lowflow, un-oriented baseline) leading to a great degree of outward remodeling.

\section{Conclusions}

In conclusion, these data demonstrate that a simple and cost-effective technology for measuring spatial variations in blood velocity along individual collateral arteries can lead to novel understanding of the arteriogenic response. Trans-illumination LSF provides the first method available for imaging these vessels at the required resolution in a quantitative manner. 
The minimal barriers presented by the technique should help enable wide-spread adoption to a variety of cardiovascular fluid mechanics studies in models that are currently inaccessible to intravital imaging. Using this novel application of LSF, we were able to obtain the first in vivo measurements of the hemodynamic signals that initiate arteriogenesis in developing collateral arteries. By incorporating this dataset into the study of endothelial cell responses to shear stress, we conclude that understanding how altered shear stress regulates arteriogenesis requires assessing and incorporating the pre-existing shear stress magnitude and direction. Incorporating these pre-existing environmental cues to more fully activate the arteriogenic program may provide a stronger or novel activation of the arteriogenic signaling pathways from which to tease out critical regulators of the arteriogenic response and help identify better therapeutic arteriogenesis targets.

\section{Acknowledgements:}

The authors would like to thank Dr. Stephen Turner (University of Virginia Bioinformatics Core) for help in processing and analysis of the microarray data and the University of Virginia Research Histology Core (under the direction of Sheri VanHoose) for histological tissue processing.

\section{Sources of Funding:}

This work was supported by American Heart Association awards 09PRE2060385 (JKM) and 10GRNT3490001 (RJP) and NIH grants T32-GM007267 (JKM), T32-HL007284 (JKM and JLH), and R21-HL098632 and R01-HL074082(RJP).

\section{Disclosures:}

The authors have no conflicts to report. 


\section{Chapter 5 Figures and Tables}
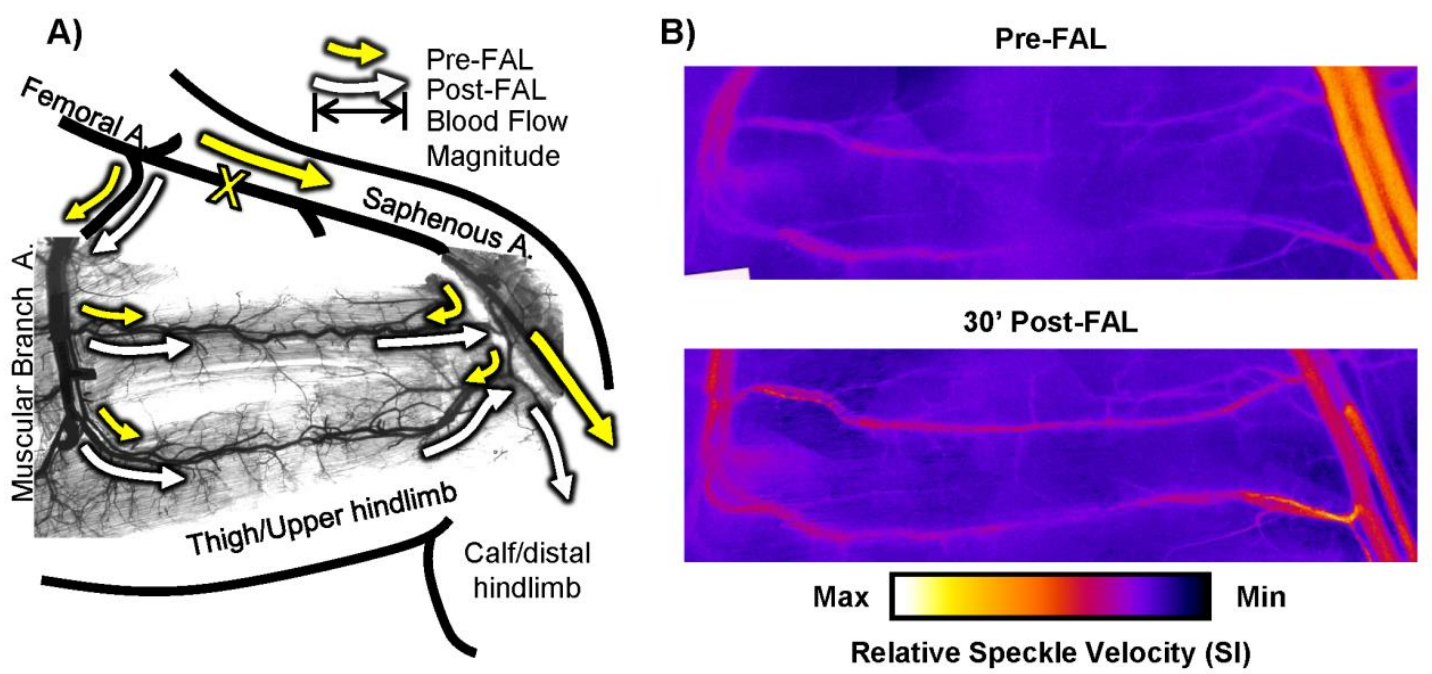

C)

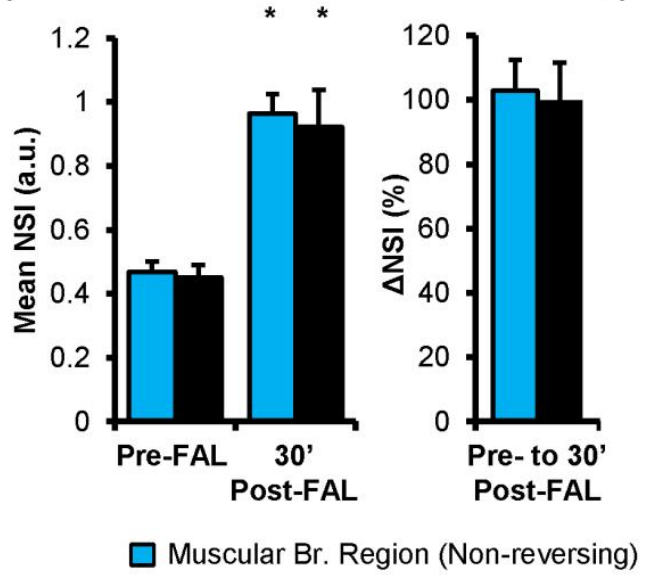

D)

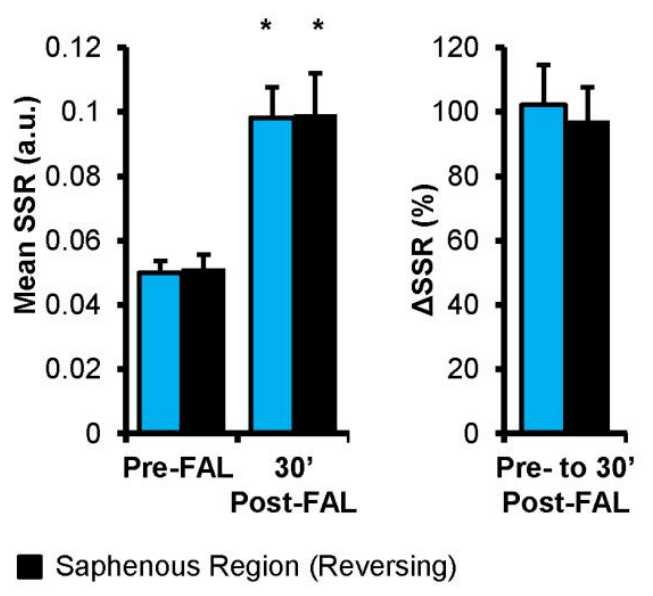

\section{Figure 5.1}

Hemodynamic changes occurring during femoral artery ligation (FAL) that drive arteriogenesis. A) Schematic of the primary gracilis adductor collateral pathways superimposed upon an image of a vascular cast of the remodeled collateral arteries in a gracilis muscle 7 days post-FAL. Arrows indicate the predicted direction and magnitude of blood flow both pre- (yellow) and post- (white) FAL (denoted by "X"). Flow direction reverses from pre- to post-FAL in the collateral region nearest the saphenous artery. These changes were confirmed by observation of circulating fluorescent microspheres $(n=4)$. B) High resolution maps of blood velocity before and 30 minutes after FAL produced from transillumination LSF. The change from low flow at the entrance regions with a point of convergence in the central regions preFAL to high flow continuously across the collateral arteries was apparent in all experiments $(n=10)$. C) Bar graphs of relative velocity signal (NSI) within the muscular branch (non-reversing) and saphenous artery (reversing) entrance regions. D) Bar graphs of relative shear stress (SSR). * indicates $p<0.05$ between pre- and post-FAL within the same region ( $\mathrm{n}=10$ mice). 

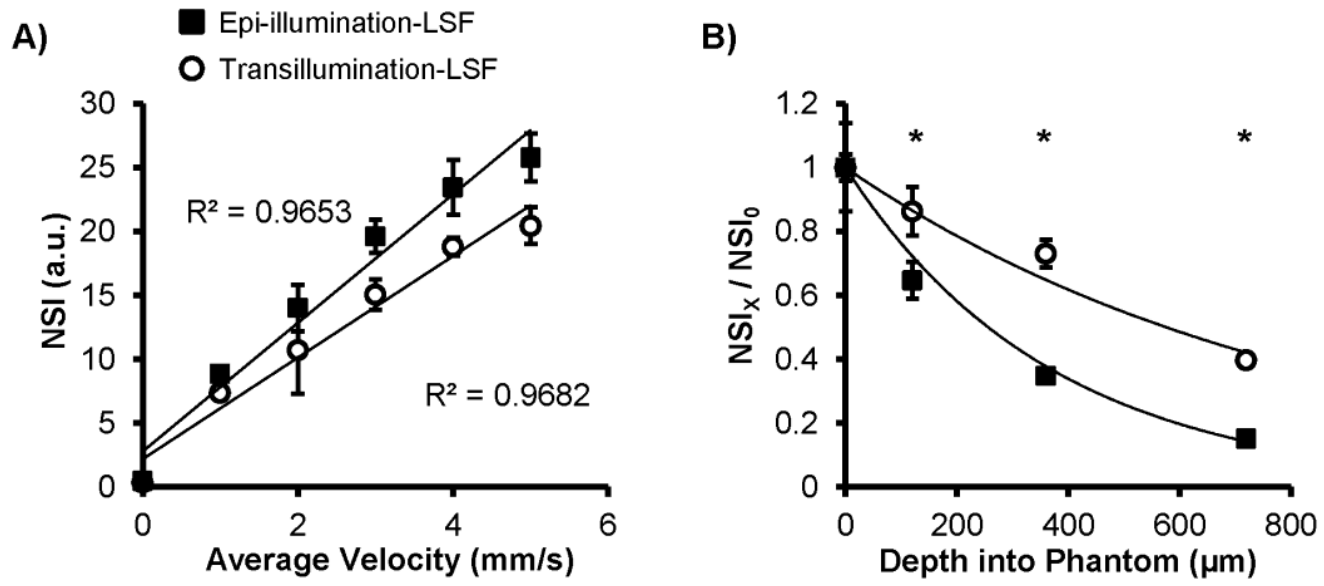

\section{Figure 5.2}

Trans-illumination maintains linear relationship between NSI and increases sensitivity to deeper velocity signals in vitro. A) Line graph of NSI for both epi-illumination (closed squares) and trans-illumination (open circles) configurations ( $n=5$ independent trials) as a function of known blood velocity. Both configurations demonstrated similar and highly linear relationships. B) Graph of speckle velocity ( $\mathrm{NSI}_{\mathrm{x}}$ ), normalized to surface speckle velocity $\left(\mathrm{NSI}_{0}\right)$, as a function of depth for epi-illumination and transillumination fit with an exponential relationship (line). * indicates $p<0.05$ between epi- and transillumination at the same depth. 

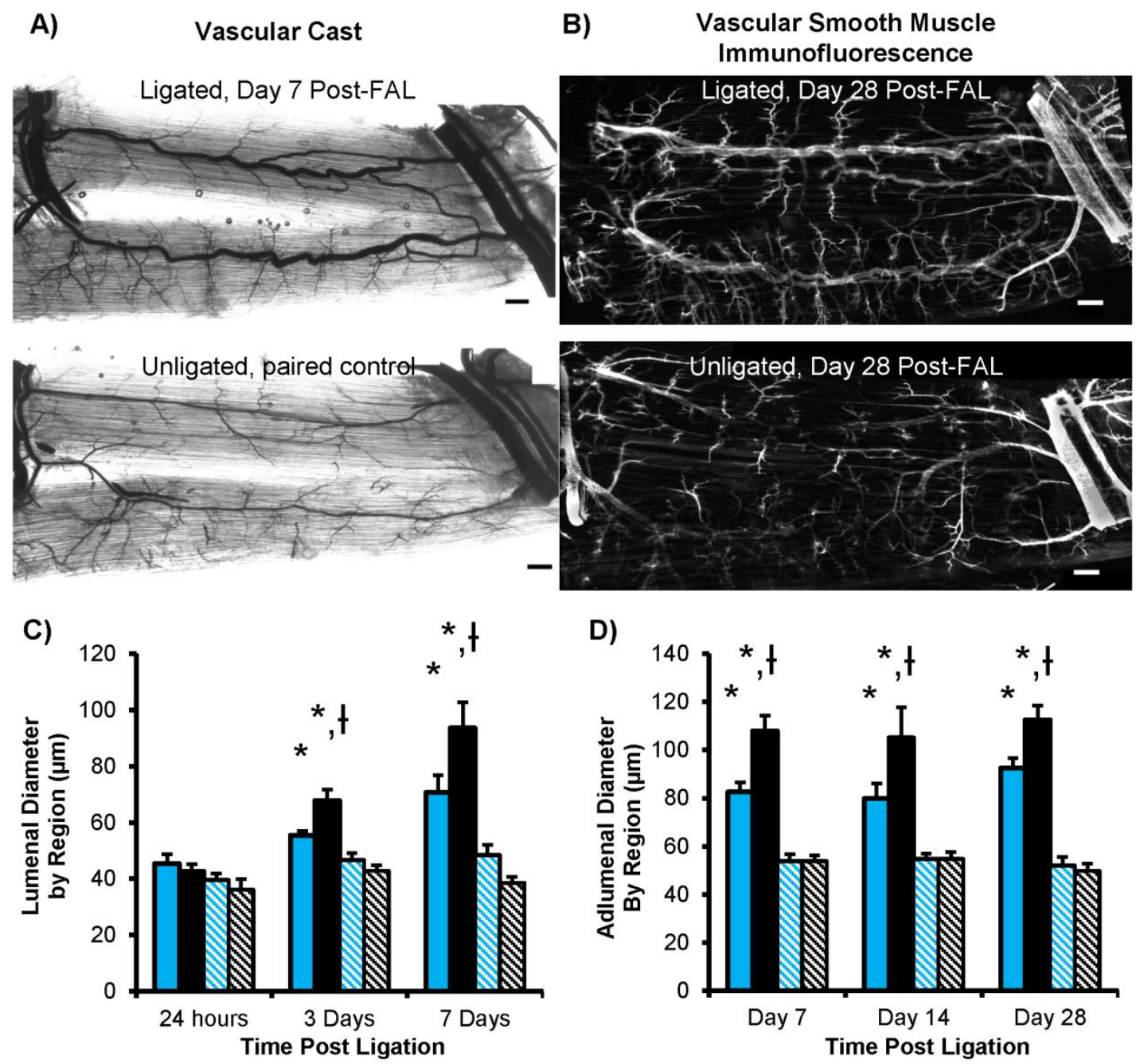

Saphenous Ligated (Reversing) \$ Saphenous Unligated Muscular Ligated (Non-reversing) \$ Muscular Unligated

\section{Figure 5.3}

Arteriogenesis is enhanced within flow reversed regions of the gracilis collateral network after FAL. A, B) Representative images of the remodeled gracilis collateral network (and the paired unligated control) obtained by either vascular casting or whole mount immunofluorescence for vascular smooth muscle (smooth muscle a-actin). C) Bar graph of collateral artery lumenal diameter at both the flow-reversing (saphenous, black) and non-reversing (muscular branch, blue) entrance regions in the remodeled collateral arteries (ligated, solid) and their unligated (sham operated, hatched) control muscles ( $n=6-7$ mice per day). D) Bar graph of adluminal collateral artery diameter, which showed similar enhanced outward remodeling in the flow reversed regions at all time points out to 28 days post-FAL ( $n=6-10$ mice per day). ${ }^{*}$ indicates $p<0.05$ between ligated and unligated diameter within the given region at the specified time-point; + indicates $p<0.05$ between regions (saphenous versus muscular) within the given treatment (ligated or unligated) at the specified time-point. 
A)

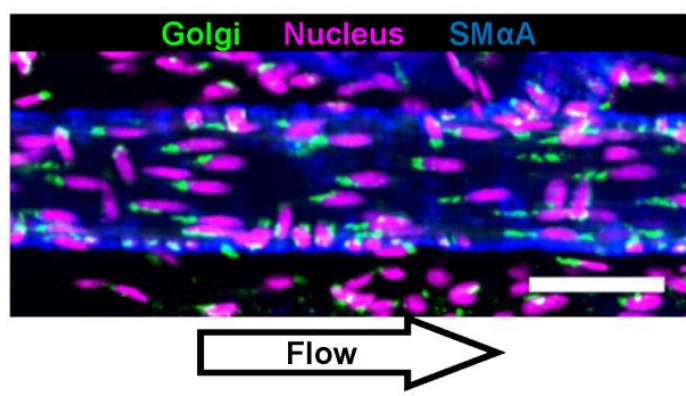

C)

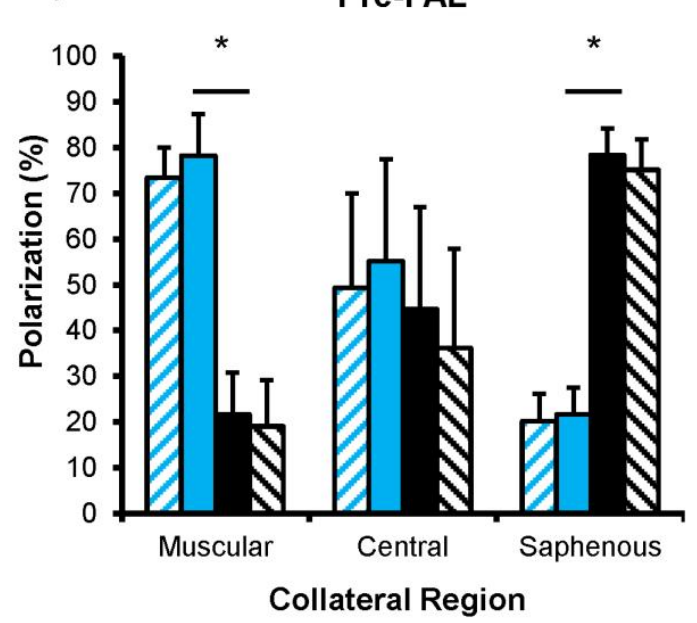

B)

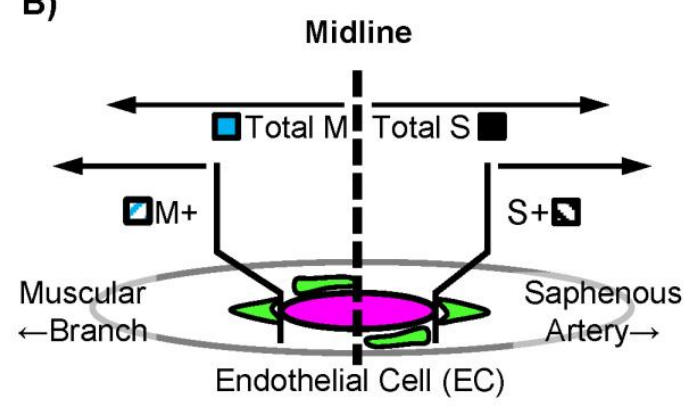

D)

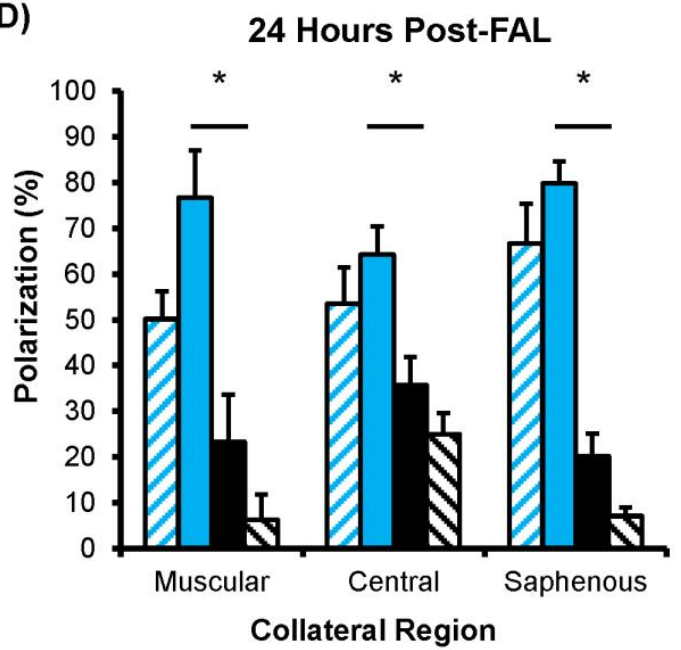

\section{Figure 5.4}

Collateral artery endothelial cell planar polarization is sensitive to directional change in shear stress induced by FAL. A) Representative segment of a collateral region used to determine planar polarization (muscular branch/non-reversing region) with arrow indicating direction of blood flow. Note the upstream position of the golgi apparatus (GA, identified by anti-giantin, green) with respect to the nucleus (Draq5, magenta) (scale bar is $25 \mu \mathrm{m}$ ). B) Diagram depicting the method used to quantify the degree of polarization. Total (hatched bars) GA polarization was classified as past the midline of the nucleus toward either the saphenous ( $\mathrm{S}$, black) or muscular branch ( $\mathrm{M}$, blue) arteries. GA completely up/down stream of the nucleus was classified as strongly polarized (+, solid bars). C) Bar graph of pre-FAL EC planar polarization. ECs in the entrance regions show upstream polarization, while the central region shows no significant polarization ( $n=4)$. D) Bar graph of 24 hour post FAL EC planar polarization. ECs in all regions show upstream polarization toward the muscular branch entrance region $(n=4)$. ${ }^{*}$ indicates $p<0.05$ between total (hatched) muscular branch versus saphenous polarization within the given region. 
A)

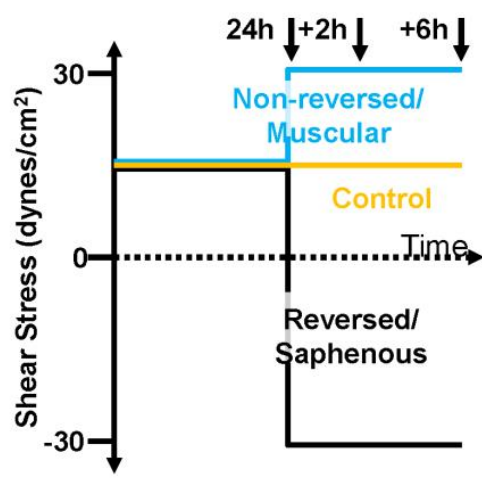

C)

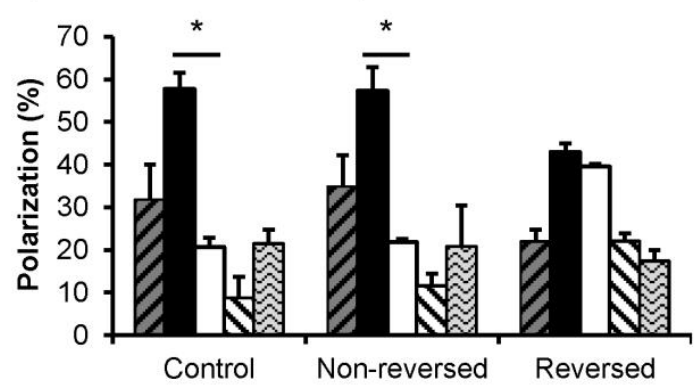

+6hrs post "FAL"

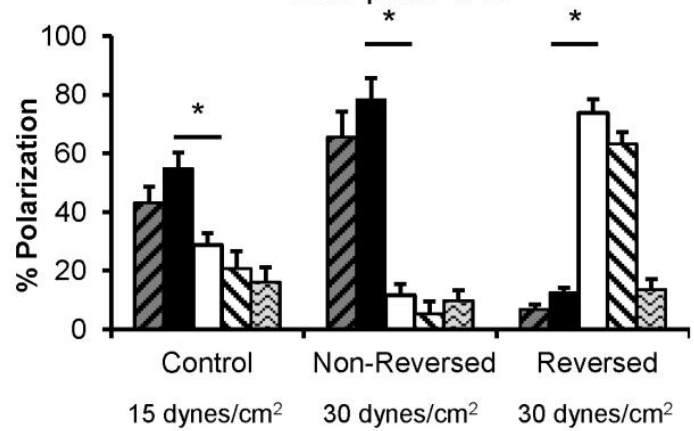

Post "FAL" Shear Stress

Upstream+ Upstream Downstream Downstream+ Dispersed

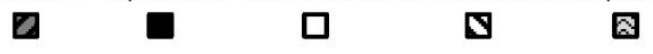

Peri-nuclear Golgi position

wrt. Pre-conditioning Shear Direction
B)

Shear $\frac{\left(\mathrm{dyn} / \mathrm{cm}^{2}\right) \rightarrow \text { Direction }}{\text { Time }}$

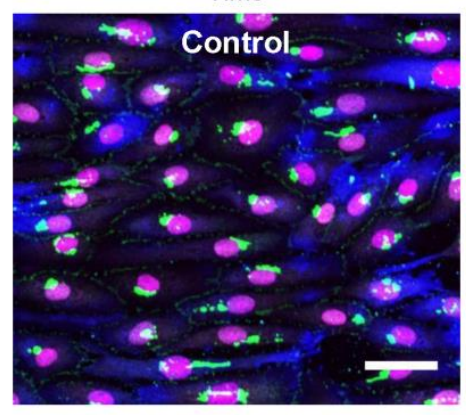

$$
\underset{\text { Pre }}{\stackrel{15 \rightarrow \mathrm{CW}}{\text { Post }}} \quad \frac{15 \rightarrow \mathrm{CW}}{}
$$
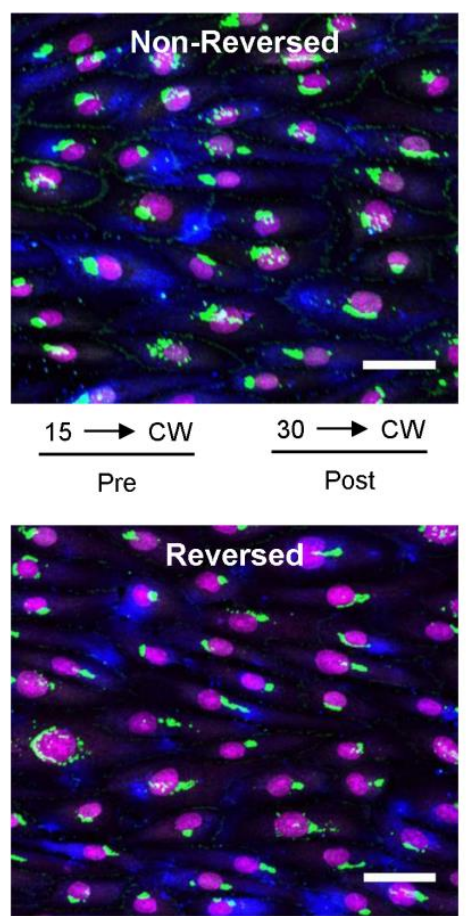

$$
\underset{\text { Pre }}{\stackrel{15 \rightarrow \mathrm{CW}}{\text { Post }}} \quad \frac{30 \longleftarrow \mathrm{cCW}}{}
$$

\section{Figure 5.5}

HUVEC planar polarization is sensitive to directional change in shear stress induced by simulated "FAL". A) Schematic depicting shear stress conditions applied to HUVECs to simulate reversing and nonreversing regions. HUVECs were preconditioned to shear direction (clockwise, CW) for 24 hours at 15 dynes $/ \mathrm{cm}^{2}$ and then increased (or kept constant, control) to $30 \mathrm{dynes} / \mathrm{cm}^{2}$ in the same or opposite (CCW) direction. B) Representative images of HUVEC planar polarization assessed by GA perinuclear position (see Figure 5.4A) after 6 hours post-"FAL". Polarization was classified with respect to pre-conditioned (CW) shear direction. GA that were circum-nuclear and without preferential orientation were classified as dispersed. C) Bar graph of HUVEC planar polarization at 2 hours after simulated FAL. D) Bar graph of HUVEC planar polarization at 6 hours after simulated FAL. * indicates $p<0.05$ between total (hatched) upstream versus downstream polarization within the given flow condition. 
A)

All Transcript FDR $<0.1$

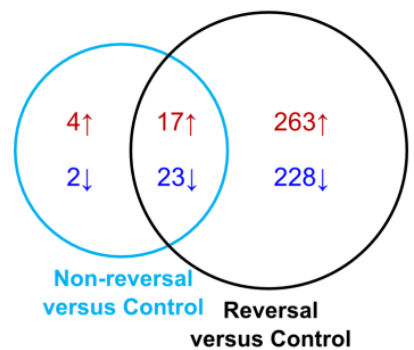

C) $\quad \mathrm{RvC} \quad \mathrm{NvC}$

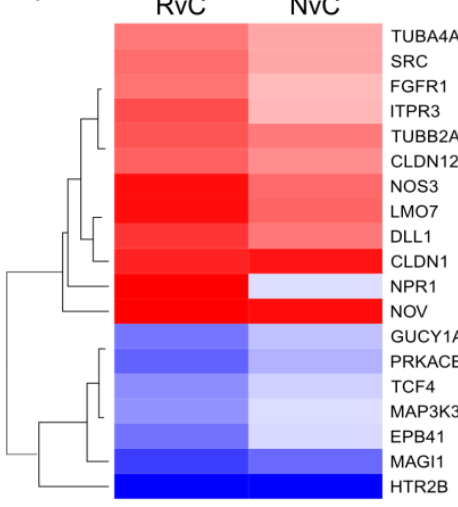

Cell-Cell Junction Signaling

E) $\quad \mathrm{RvC} \quad \mathrm{NvC}$
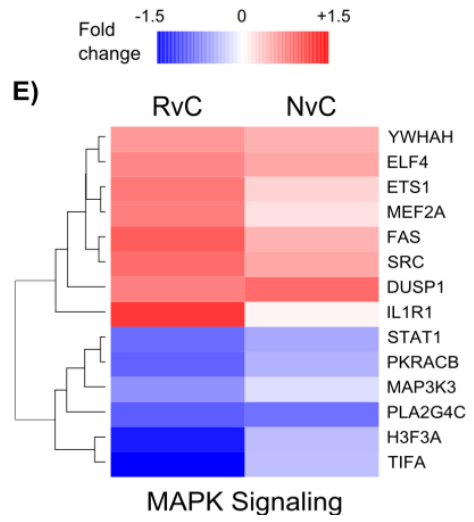

B)

All Transcripts FDR $<0.1$

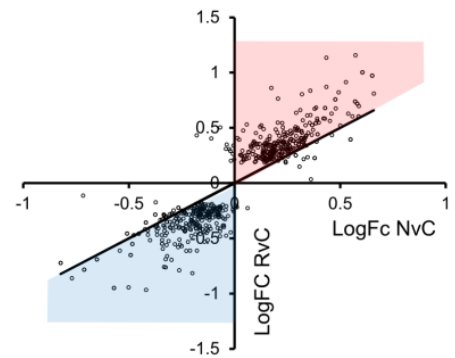

D)

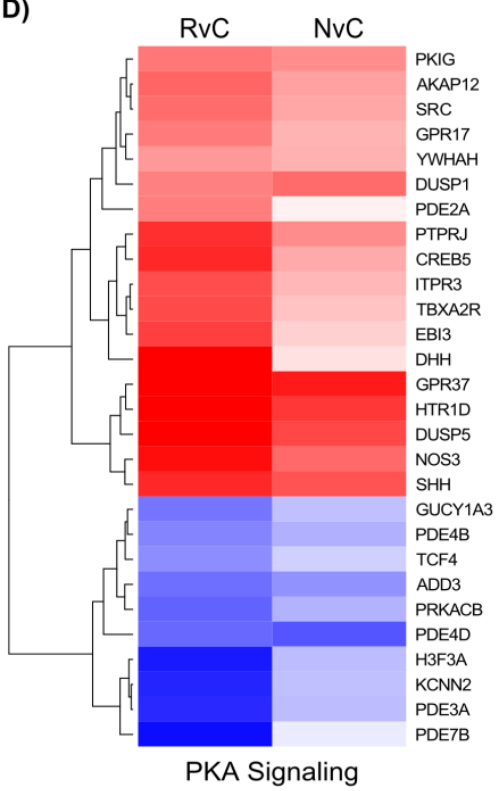

F)

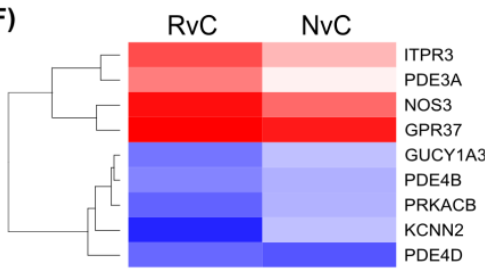

PDE Signaling

\section{Figure $\mathbf{5 . 6}$}

Addition of reversed directional stimulus enhances the arteriogenic expression pattern seen with an increase in shear stress. A) Venn diagram of the overlapping and non-overlapping genes showing significant $(\mathrm{FDR}<0.1)$ regulation with a non-reversed increase in shear stress versus control $(\mathrm{NvC})$ conditions and those significantly altered with an increase in shear stress with reversed direction versus control (RvC). B) Scatterplot showing gene expression changes between RvC and NvC conditions. A similar pattern of expression (either up in both or down in both) was observed, but a greater degree of enhancement was present in the reversed shear stress condition (red region, greater up-regulation in $\mathrm{RvC}$; blue region, greater down-regulation in RvC; line, equal RvC and $\mathrm{NvC}$ ). C-F) Heatmaps of the involved genes grouped along significantly altered canonical pathways (cell-cell junctional signaling, protein kinase A signaling, MAP kinase signaling, and phosphodiesterase signaling) activated in both $\mathrm{RvC}$ and NvC data (see online Table IV).Identical patterns of activation were observed between the conditions but to a greater degree in the reversed flow condition. 


\section{Table 5.1}

Table 5.1. Comparison of microarray genes to RT-PCR analyses for selected genes known to mediate arteriogenesis

\begin{tabular}{|c|c|c|c|c|c|c|}
\hline \multirow[b]{2}{*}{$\begin{array}{l}\text { Entrez } \\
\text { Symbol }\end{array}$} & \multirow[b]{2}{*}{ Gene Name } & \multirow[b]{2}{*}{ Comparison } & \multirow{2}{*}{$\begin{array}{c}\text { RT-PCR } \\
\text { Log }_{2} \text { Fold } \\
\text { Change }\end{array}$} & \multicolumn{3}{|c|}{ Microarray } \\
\hline & & & & $\begin{array}{l}\log _{2} \text { Fold } \\
\text { Change }\end{array}$ & $p$-value & FDR \\
\hline NOS3 & $\begin{array}{l}\text { nitric oxide synthase } 3 \\
\text { (endothelial cell) }\end{array}$ & $\begin{array}{l}\mathrm{RvC} \\
\mathrm{NvC}\end{array}$ & $\begin{array}{c}1.17 \pm 0.49 * t \\
0.30 \pm 0.44\end{array}$ & $\begin{array}{l}0.47 \pm 0.13 \\
0.29 \pm 0.13\end{array}$ & $\begin{array}{l}2.03 e-5 \\
1.09 e-3\end{array}$ & $\begin{array}{l}0.011 \\
0.207\end{array}$ \\
\hline ICAM1 & $\begin{array}{l}\text { intercellular adhesion } \\
\text { molecule } 1\end{array}$ & $\begin{array}{l}\mathrm{RvC} \\
\mathrm{NvC}\end{array}$ & $\begin{array}{c}0.92 \pm 0.25^{*} \mathrm{t} \\
0.49 \pm 0.44\end{array}$ & $\begin{array}{l}0.15 \pm 0.12 \\
0.10 \pm 0.12\end{array}$ & $\begin{array}{l}0.031 \\
0.114\end{array}$ & $\begin{array}{l}0.331 \\
0.640\end{array}$ \\
\hline VCAM1 & $\begin{array}{l}\text { vascular cell adhesion } \\
\text { molecule } 1\end{array}$ & $\begin{array}{l}\mathrm{RvC} \\
\mathrm{NvC}\end{array}$ & $\begin{array}{c}0.23 \pm 0.78 \\
-0.67 \pm 0.43 \text { * }\end{array}$ & $\begin{array}{l}-0.22 \pm 0.29 \\
-0.66 \pm 0.29\end{array}$ & $\begin{array}{c}0.161 \\
9.86 \mathrm{e}-4\end{array}$ & $\begin{array}{l}0.641 \\
0.203\end{array}$ \\
\hline KLF2 & $\begin{array}{l}\text { Kruppel-like factor } 2 \\
\text { (lung) }\end{array}$ & $\begin{array}{l}\mathrm{RvC} \\
\mathrm{NvC}\end{array}$ & $\begin{array}{l}0.84 \pm 0.31 * t \\
0.43 \pm 0.25 \text { * }\end{array}$ & $\begin{array}{l}0.33 \pm 0.11 \\
0.26 \pm 0.11\end{array}$ & $\begin{array}{l}1.30 \mathrm{e}-4 \\
7.47 \mathrm{e}-4\end{array}$ & $\begin{array}{l}0.025 \\
0.176\end{array}$ \\
\hline KLF4 & $\begin{array}{l}\text { Kruppel-like factor } 4 \\
\text { (gut) }\end{array}$ & $\begin{array}{l}\mathrm{RvC} \\
\mathrm{NvC}\end{array}$ & $\begin{array}{l}0.95 \pm 0.35 \text { * } \\
0.53 \pm 0.33 \text { * }\end{array}$ & $\begin{array}{l}0.47 \pm 0.13 \\
0.26 \pm 0.13\end{array}$ & $\begin{array}{l}1.86 \text { e-5 } \\
2.06 \text { e-3 }\end{array}$ & $\begin{array}{l}0.010 \\
0.261\end{array}$ \\
\hline SELE & selectin $E$ & $\begin{array}{l}\mathrm{RvC} \\
\mathrm{NvC}\end{array}$ & $\begin{array}{l}-0.86 \pm 0.63 * \\
-0.94 \pm 0.51 \text { * }\end{array}$ & $\begin{array}{l}-0.86 \pm 0.32 \\
-0.77 \pm 0.32\end{array}$ & $\begin{array}{l}2.57 \mathrm{e}-4 \\
6.35 \mathrm{e}-4\end{array}$ & $\begin{array}{l}0.032 \\
0.157\end{array}$ \\
\hline CCL2 & $\begin{array}{l}\text { chemokine (C-C motif) } \\
\text { ligand } 2\end{array}$ & $\begin{array}{l}\mathrm{RvC} \\
\mathrm{NvC}\end{array}$ & $\begin{array}{l}-0.69 \pm 0.37^{*} \\
-0.38 \pm 0.32\end{array}$ & $\begin{array}{l}-0.19 \pm 0.11 \\
-0.08 \pm 0.11\end{array}$ & $\begin{array}{l}5.53 \text { e-3 } \\
0.168\end{array}$ & $\begin{array}{l}0.153 \\
0.706\end{array}$ \\
\hline
\end{tabular}

Data represents RT-PCR and microarray analysis of $n=4 /$ group. $R v C$, reversal versus control. NvC non-reversal versus control. *, indicates $p<0.05$ significance versus control RT-PCR, $t$, indicates significance $p<0.05$ of reversal v nonreversal by RT-PCR expression levels Uncertainty in PCR is mean +/- standard deviation for microarray mean $+/-95 \%$ confidence 


\section{Table 5.2}

Table 5.2. Predicted upstream regulators of gene expression patterns seen with reversing and non-reversing increased shear stress

\begin{tabular}{|c|c|c|c|c|}
\hline \multirow{3}{*}{ Upstream Regulator } & \multicolumn{2}{|c|}{ Reversal v Control } & \multicolumn{2}{|c|}{ Non-reversal v Control } \\
\hline & Activation Z-Score & $p$-value overlap & Activation Z-Score & $p$-value overlap \\
\hline & \multicolumn{4}{|c|}{ A. NFKB Pathway } \\
\hline $\begin{array}{c}\text { phorbol myristate acetate } \\
\text { (activator PKC and NFKB) }\end{array}$ & 2.767 & 1.35E-03 & & \\
\hline NFKBIA & 2.273 & $2.56 \mathrm{E}-02$ & & 4.95E-02 \\
\hline RELA & 1.85 & $1.54 \mathrm{E}-02$ & & \\
\hline NFkB (complex) & 1.47 & 3.99E-03 & & \\
\hline IKBKB & 1.272 & $6.94 \mathrm{E}-06$ & & $1.22 \mathrm{E}-02$ \\
\hline \multicolumn{5}{|l|}{ B. MAPK Pathways } \\
\hline Mek (group) & 2.215 & $3.50 \mathrm{E}-02$ & & \\
\hline RAF1 & 2.037 & $2.62 \mathrm{E}-05$ & & $9.31 \mathrm{E}-04$ \\
\hline ERK1/2 & 1.912 & 3.05E-02 & & \\
\hline MAPK14 & 1.793 & $2.50 \mathrm{E}-02$ & & $3.38 \mathrm{E}-02$ \\
\hline MAP3K1 & 1.513 & $1.65 \mathrm{E}-02$ & & $6.70 \mathrm{E}-03$ \\
\hline ERK & 1.418 & $5.74 \mathrm{E}-03$ & & \\
\hline P38 MAPK & 1.403 & $2.72 \mathrm{E}-04$ & & \\
\hline $\begin{array}{l}\text { U0126 } \\
\text { (inhibitor of MEK 1/2) }\end{array}$ & -2.336 & $2.24 \mathrm{E}-05$ & & \\
\hline $\begin{array}{l}\text { PD98059 } \\
\text { (inhibitor of MEK 1) }\end{array}$ & -3.215 & $6.65 E-05$ & -0.577 & 1.91E-02 \\
\hline \multicolumn{5}{|l|}{ C. Growth Factors } \\
\hline HGF & 5.243 & $1.87 \mathrm{E}-13$ & & \\
\hline Vegf (group) & 4.955 & $1.81 \mathrm{E}-15$ & & \\
\hline EGF & 2.407 & 7.47E-05 & & \\
\hline PDGF BB & 2.33 & $1.78 \mathrm{E}-02$ & & 1.76E-02 \\
\hline FGF1 & 2.18 & 8.10E-03 & & \\
\hline EGR2 & 2.178 & $1.41 \mathrm{E}-02$ & & \\
\hline FGF2 & 2.11 & $1.68 \mathrm{E}-05$ & & \\
\hline TGFBR2 & 1.969 & $3.76 \mathrm{E}-02$ & & \\
\hline ERBB2 & 1.849 & $1.55 \mathrm{E}-03$ & & \\
\hline VEGFA & 1.84 & $1.74 \mathrm{E}-04$ & & \\
\hline TGFB3 & 1.134 & $5.48 \mathrm{E}-03$ & & \\
\hline Tgf beta (group) & 1.07 & $9.46 \mathrm{E}-03$ & & \\
\hline MET & 1 & 4.26E-03 & & \\
\hline $\begin{array}{l}\text { tyrphostin AG } 1478 \\
\text { (inhibitor of EGFR1) }\end{array}$ & -1.04 & $1.40 \mathrm{E}-02$ & & \\
\hline CTGF & -1.134 & 2.39E-03 & & $1.01 E-02$ \\
\hline \multicolumn{5}{|l|}{ D. PI3K/AKT Pathway } \\
\hline Akt & 1.941 & $3.40 \mathrm{E}-02$ & & \\
\hline PI3K (complex) & 1.714 & $1.43 \mathrm{E}-02$ & & \\
\hline $\mathrm{Ca} 2+$ & 1.191 & $4.45 \mathrm{E}-07$ & & \\
\hline $\begin{array}{l}\text { LY294002 } \\
\text { (inhibitor of PI3K) }\end{array}$ & -1.12 & $2.25 \mathrm{E}-04$ & & 4.99E-02 \\
\hline $\begin{array}{l}\text { wortmannin } \\
\text { (inhibitor of PI3K) }\end{array}$ & -2.007 & 8.01E-02 & & \\
\hline \multicolumn{5}{|l|}{ E. Cytokines } \\
\hline IL13 & 2.493 & $1.46 \mathrm{E}-01$ & & \\
\hline CXCL12 & 1.542 & $2.26 \mathrm{E}-02$ & & \\
\hline IL3 & 1.342 & $2.20 \mathrm{E}-02$ & & \\
\hline Ifn (group) & 1.305 & 1.19E-03 & & \\
\hline IL1RN & 1.195 & 1.47E-02 & & \\
\hline IL1A & 1.115 & $2.82 \mathrm{E}-04$ & & \\
\hline TNF & 0.062 & $1.03 \mathrm{E}-10$ & 1.236 & 8.23E-05 \\
\hline IFNA1/IFNA13 & -1 & $1.52 \mathrm{E}-02$ & & \\
\hline Tnf (family) & -1.089 & $5.67 \mathrm{E}-03$ & & \\
\hline IL27 & -1.277 & 8.09E-03 & & \\
\hline IFNA2 & -1.564 & $1.20 \mathrm{E}-02$ & & \\
\hline IFNB1 & -1.665 & $2.75 \mathrm{E}-03$ & & \\
\hline TNFSF10 & -1.715 & $2.22 \mathrm{E}-02$ & & \\
\hline
\end{tabular}

All matching upstream regulators fitting into the defined groups with activation $\mid z$-score $\mid>1.0$ 


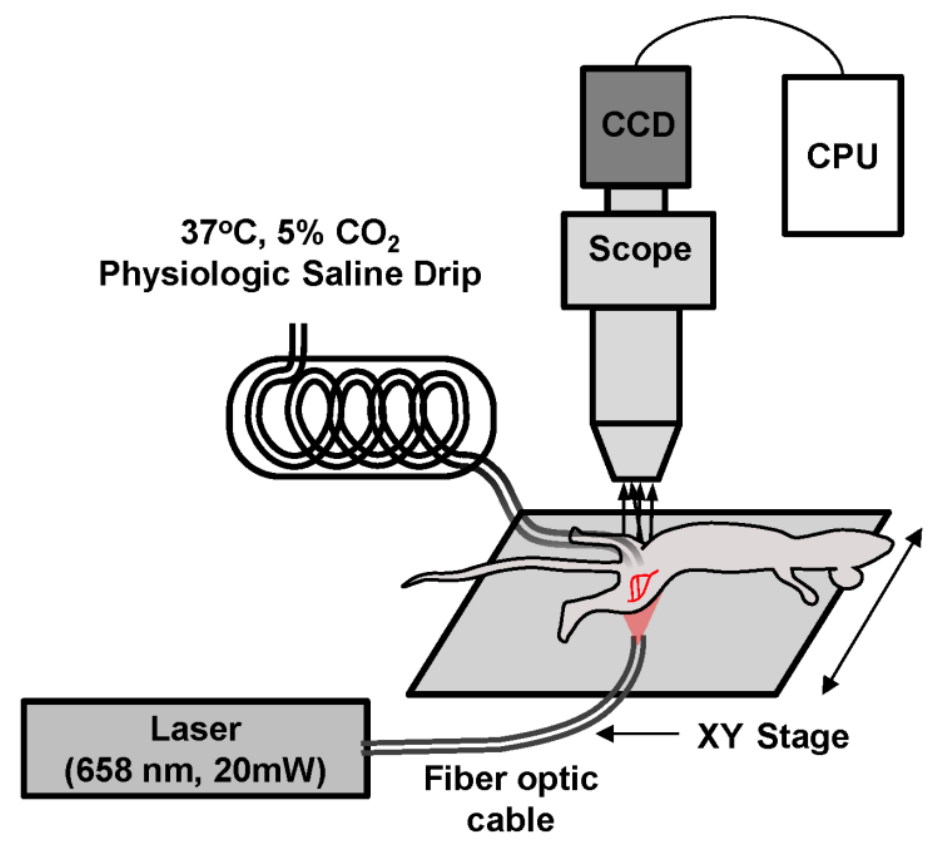

\section{Online Figure 5.I}

Arrangement for intravital imaging of collateral artery hemodynamics. The fiber optic cable was positioned for either trans-illumination or epi-illumination. For the trans-illumination configuration, the fiber optic outlet was placed on the opposite side of the leg from the exposed muscle and against the depilated skin to yield an evenly illuminated field of view. For the epi-illumination configuration, the fiber optic outlet was positioned to produce an unobstructed and even sheet of light on the surface of the exposed muscle. The given field of view was shifted using an xy-stage. Warmed physiologic saline solution deoxygenated with $5 \% \mathrm{CO}_{2}$ was perfused over the surface of the exposed muscle to maintain a constant surface temperature of $37^{\circ} \mathrm{C}$. 


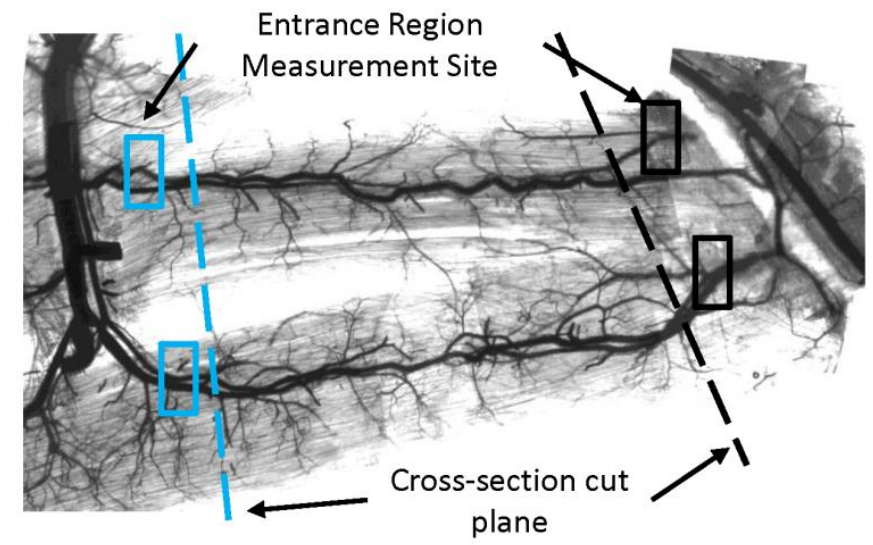

\section{Online Figure 5.II}

Demarcation of regional cross section cut planes. Boxes indicate the regions where lumenal and adluminal diameter were measured (at the first transverse arteriole branch point). Dashed lines indicate the approximate location where muscles were cut for regional analysis. 
A)

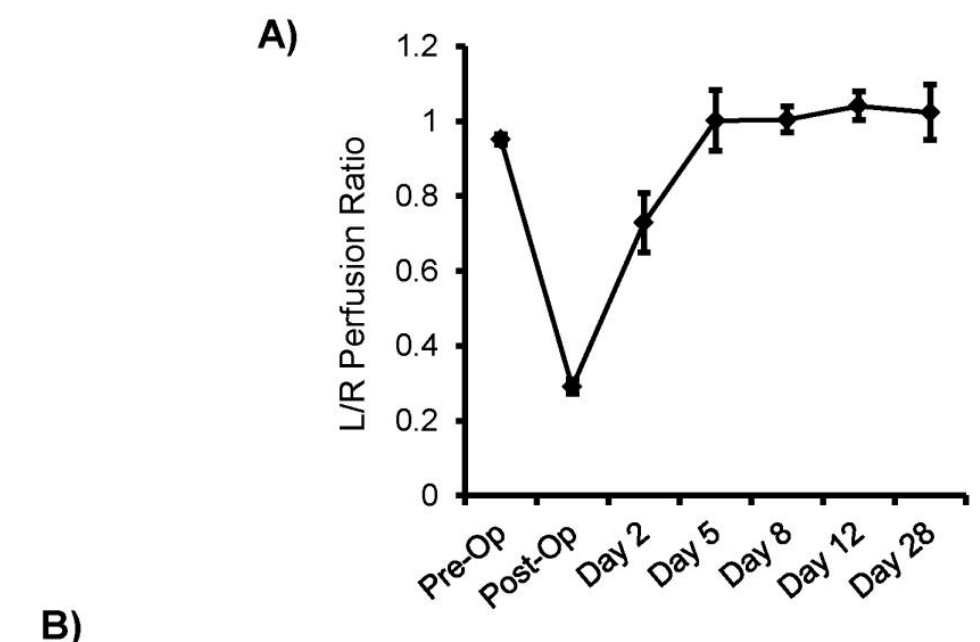

B)
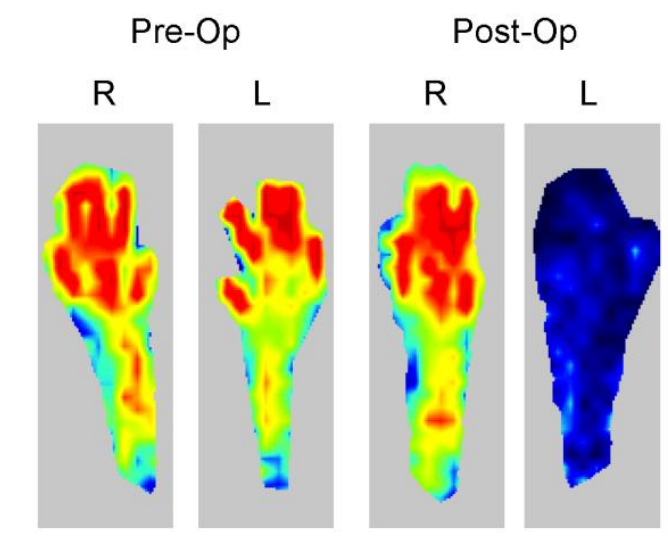

Day 2

Day 5
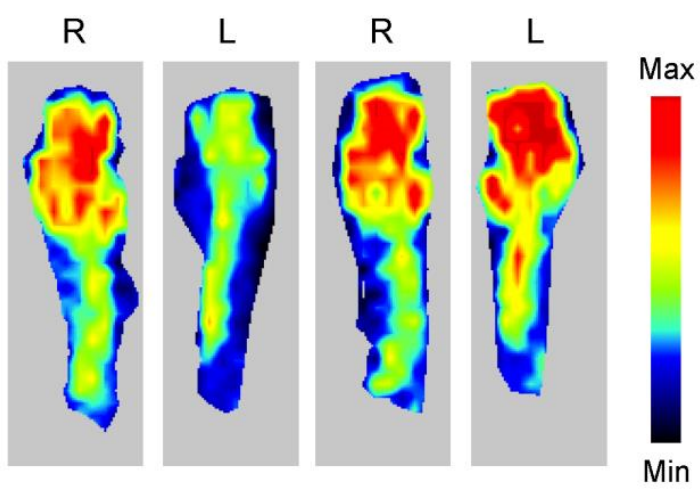

\section{Online Figure 5.III}

Laser Doppler perfusion recovery curve. A) Non-invasive laser Doppler perfusion measurements show mild ischemia that is back to baseline perfusion by day 5 post-FAL. B) Representative images of measured foot perfusion in the ligated $(L)$ and unligated $(R)$ legs. 


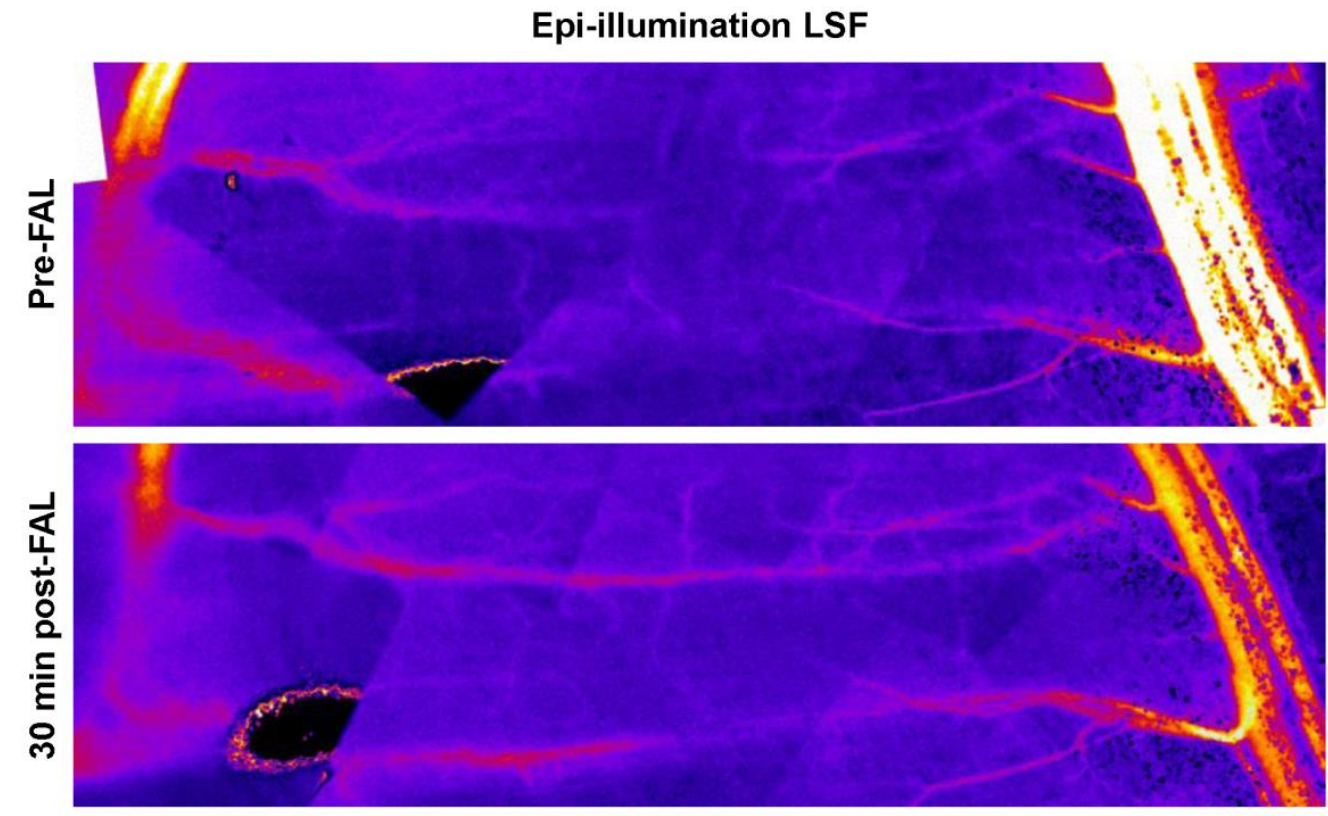

Trans-illumination LSF
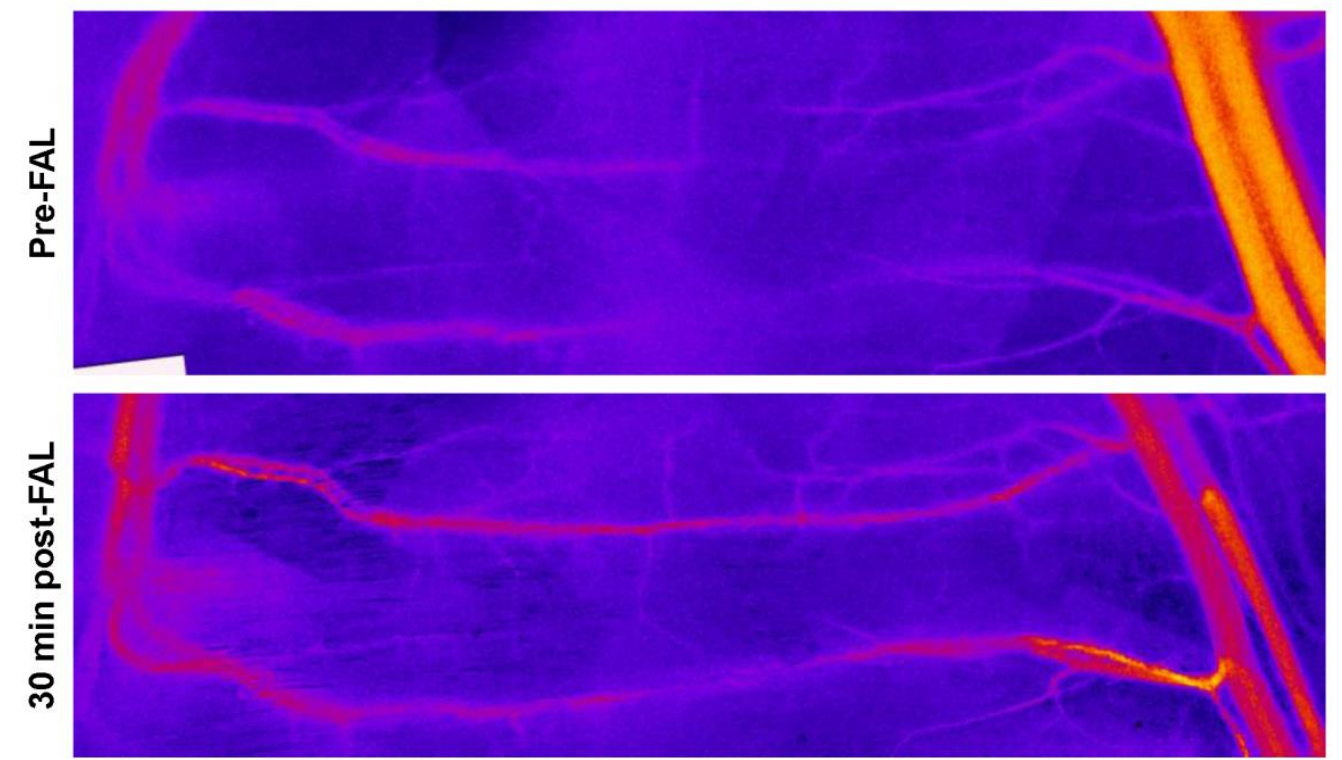

\section{Online Figure 5.IV}

Comparison of transillumination and epi-illumination based measurements of blood flow by LSF in a representative network. While epi-illumination LSF is capable of visualizing the most superficial elements of the gracilis collateral network (e.g. the saphenous artery and upstream region of the muscular branch), the epi-illumination configuration is less sensitive to the central region of the collateral network which is embedded within the muscle. Epi-illumination is also susceptible error from directly reflected light off of the tissue surface and overlying fat globules (black regions and grainy structure on the saphenous artery). Transillumination LSF has less variation in the acquired speckle signal as the collateral network varies in depth and is not susceptible to directly reflected light. 
A)

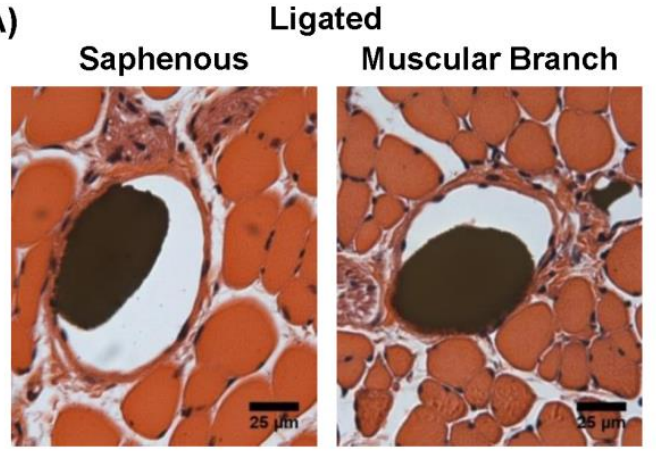

Unligated

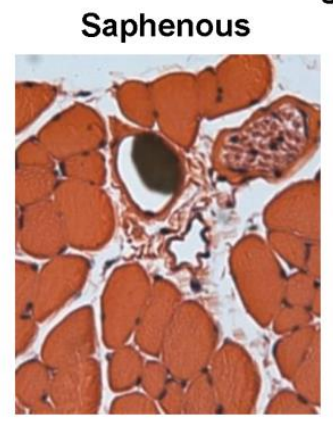

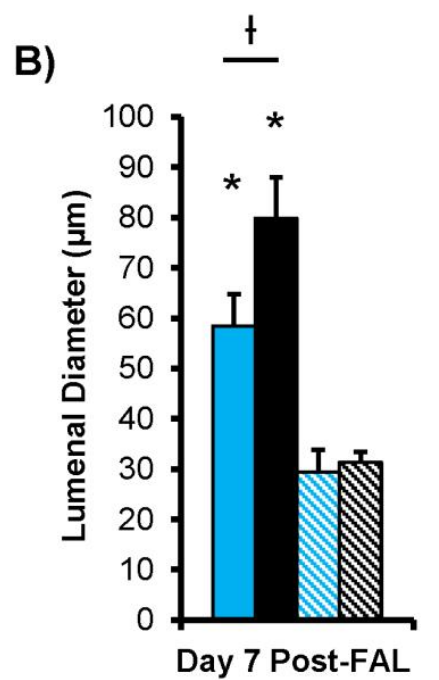

C)

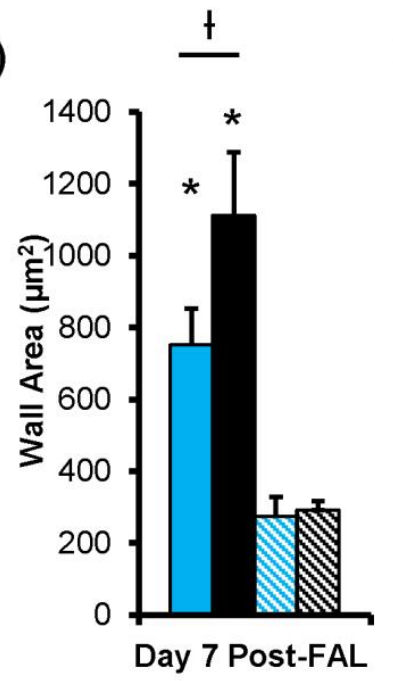

D)

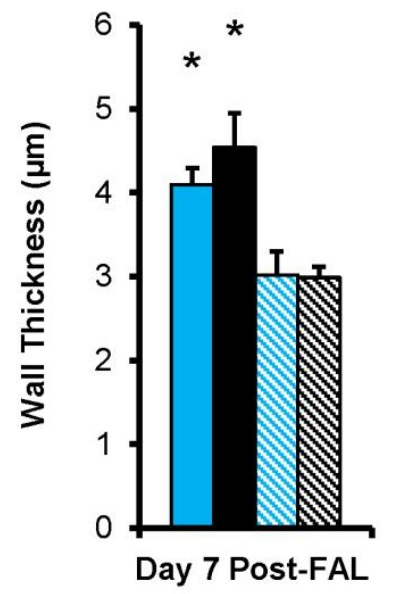

- Saphenous Ligated (Reversing)

\$ Saphenous Unligated

$\square$ Muscular Ligated (Non-reversing)

$\checkmark$ Muscular Unligated

\section{Online Figure 5.V}

Cross sectional analysis of collateral growth by region. A) Representative cross-sections of the gracilis muscle from the remodeling (ligated, solid) and control (unligated, hatched) limb within the saphenous (black) and muscular branch (blue) entrance regions were stained with H\&E. B-D) At 7 days post-FAL, quantification of total cross sectional wall area, lumenal diameter, and wall thickness show an increase in wall mass and outward remodeling within both regions versus their paired control sections, but this growth occurred to a greater degree within the flow-reversing region compared to the non-reversing region. *, indicates $\mathrm{p}<0.05$ between ligated and unligated diameter within the given region. + indicates $p<0.05$ between regions (saphenous versus muscular) within the given treatment (ligated or unligated) 


\section{A)}

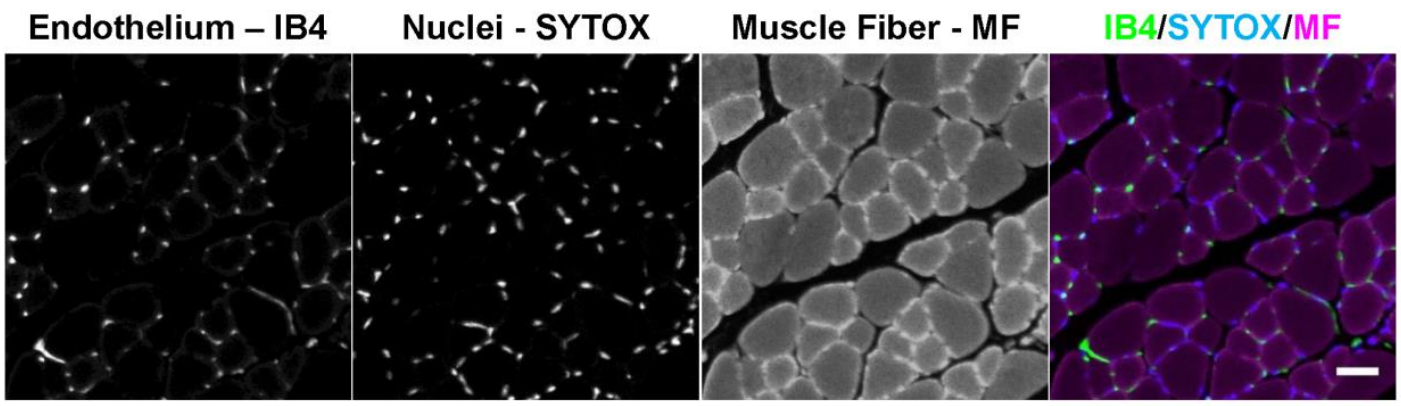

B)

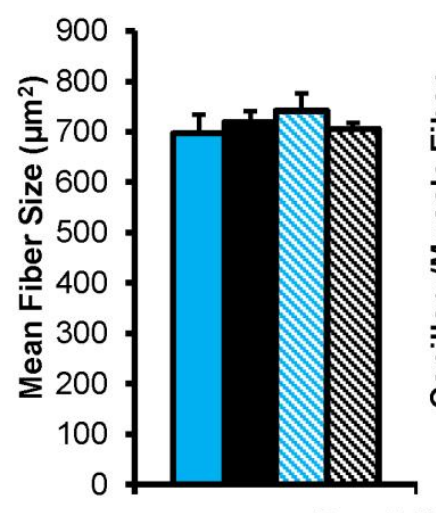

C)

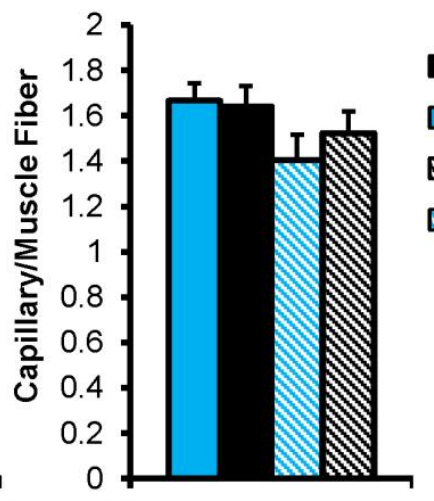

Saphenous Ligated (Reversing)

$\square$ Muscular Ligated (Non-reversing)

\$ Saphenous Unligated

Muscular Unligated

Day 7 Post-FAL

\section{Online Figure 5.VI}

Cross-sectional analysis of gracilis collateral regions reveals no evidence of functional hypoxia. A) Representative field of view of a gracilis muscle cross-section stained for capillaries (isolectin B4). Scale bar is $25 \mu \mathrm{m}$. Tissue auto-fluorescence provided endogenous contrast for identifying individual muscle fibers. B) At 7 days post-FAL, there was no evidence of muscle atrophy as determined by mean fiber size within either the muscular branch or saphenous artery entrance regions as compared to their respective unligated control. C) Similarly, there was no significant differences in capillary to muscle fiber ratio to indicate angiogenesis between ligated and unligated limbs in either region. 


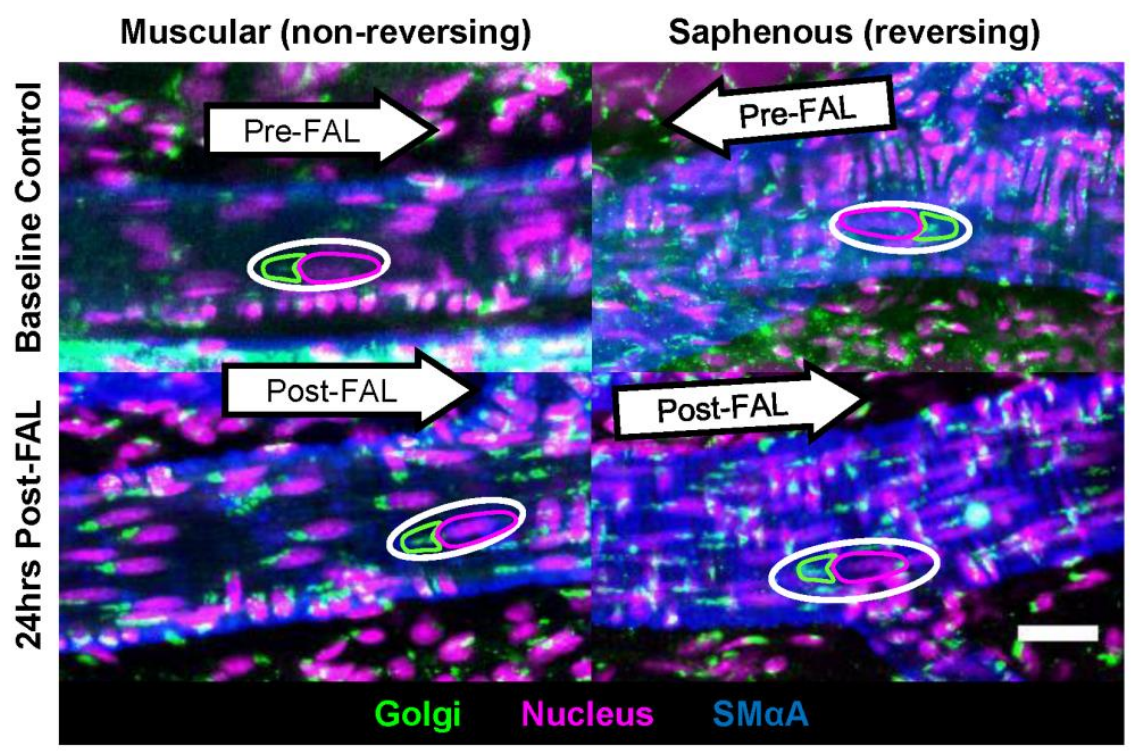

\section{Online Figure 5.VII}

Representative images of planar cell polarization orientation in reversing and non-reversing entrance regions. Images are oriented such that the muscular branch is toward the left and the saphenous artery is toward the right. Pre-FAL, the entrance regions have an upstream orientation of their Golgi apparatus (outlined in green) with respect to the nucleus (outlined in magenta) toward their respective feeding arteries. 24 hours post-FAL, both entrance regions are polarized toward the muscular branch artery. Arrows indicate anticipated direction of blood flow (scale bar is $25 \mu \mathrm{m}$ ). 
A)
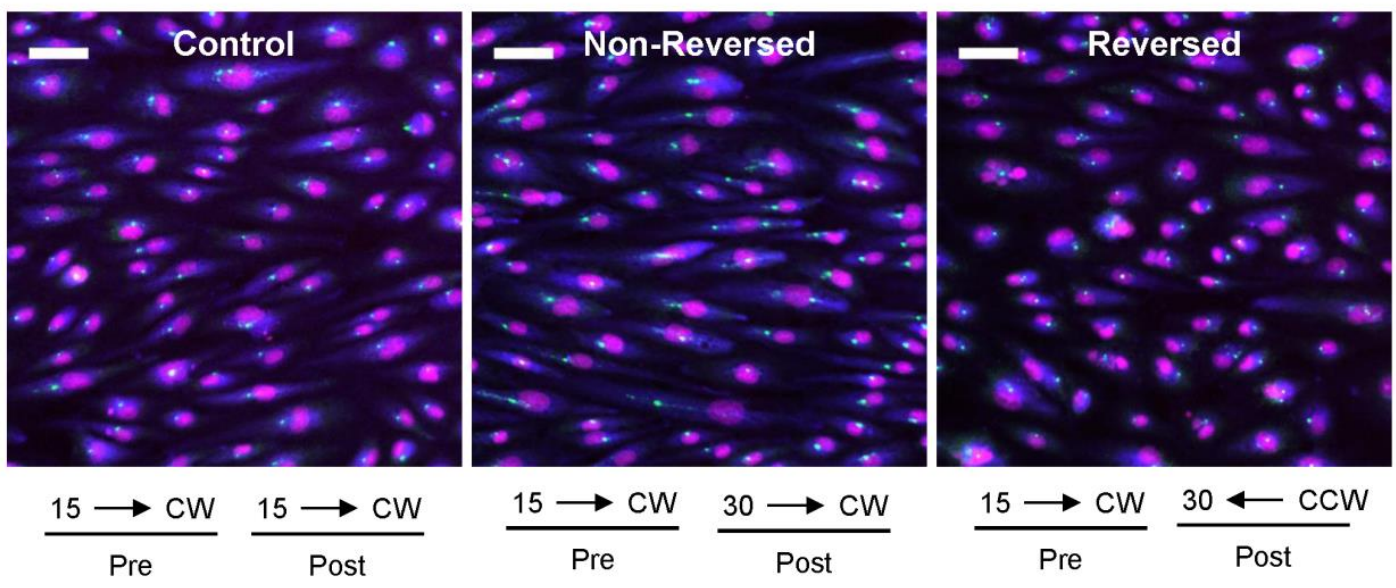

B)

+6hrs post "FAL"

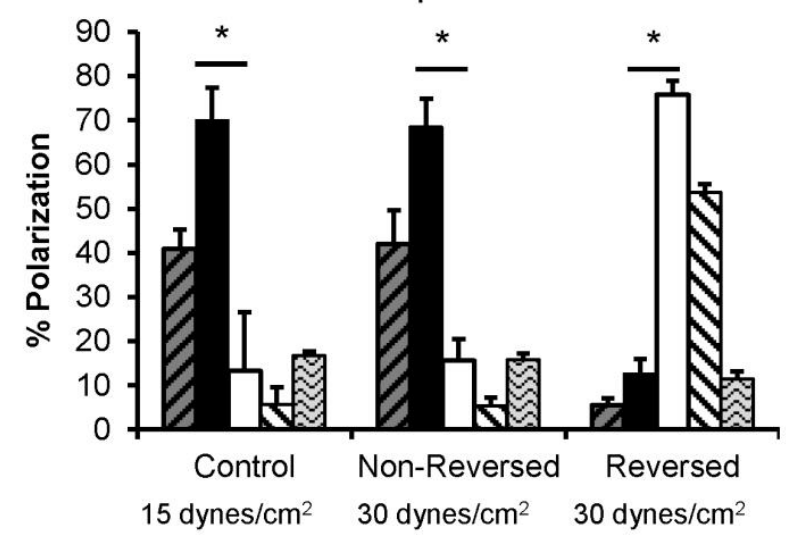

Shear $\frac{\left(\mathrm{dyn} / \mathrm{cm}^{2}\right) \rightarrow \text { Direction }}{\text { Time }}$

Post "FAL" Shear Stress

Upstream+ Upstream Downstream Downstream+ Central

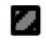

$\mathbf{\nabla}$

圆

Peri-nuclear MTOC position wrt. Pre-conditioning Shear Direction

\section{Online Figure 5.VIII}

Representative images of planar cell polarization in HUVECs using peri-nuclear position of the MTOC. Peri-nuclear position of the MTOC was also used to confirm HUVEC repolarization with reversal of shear stress. A) Representative images of each flow condition. Scale bar is $50 \mu \mathrm{m}$. B) Degree of polarization at 6 hours post-"FAL" are similar to those found using the Golgi apparatus as the polarization marker (Figure 5.5) ( $n=3-4$ per condition). *, indicates $p<0.05$ between total (solid) upstream versus downstream polarization within the given flow condition. 


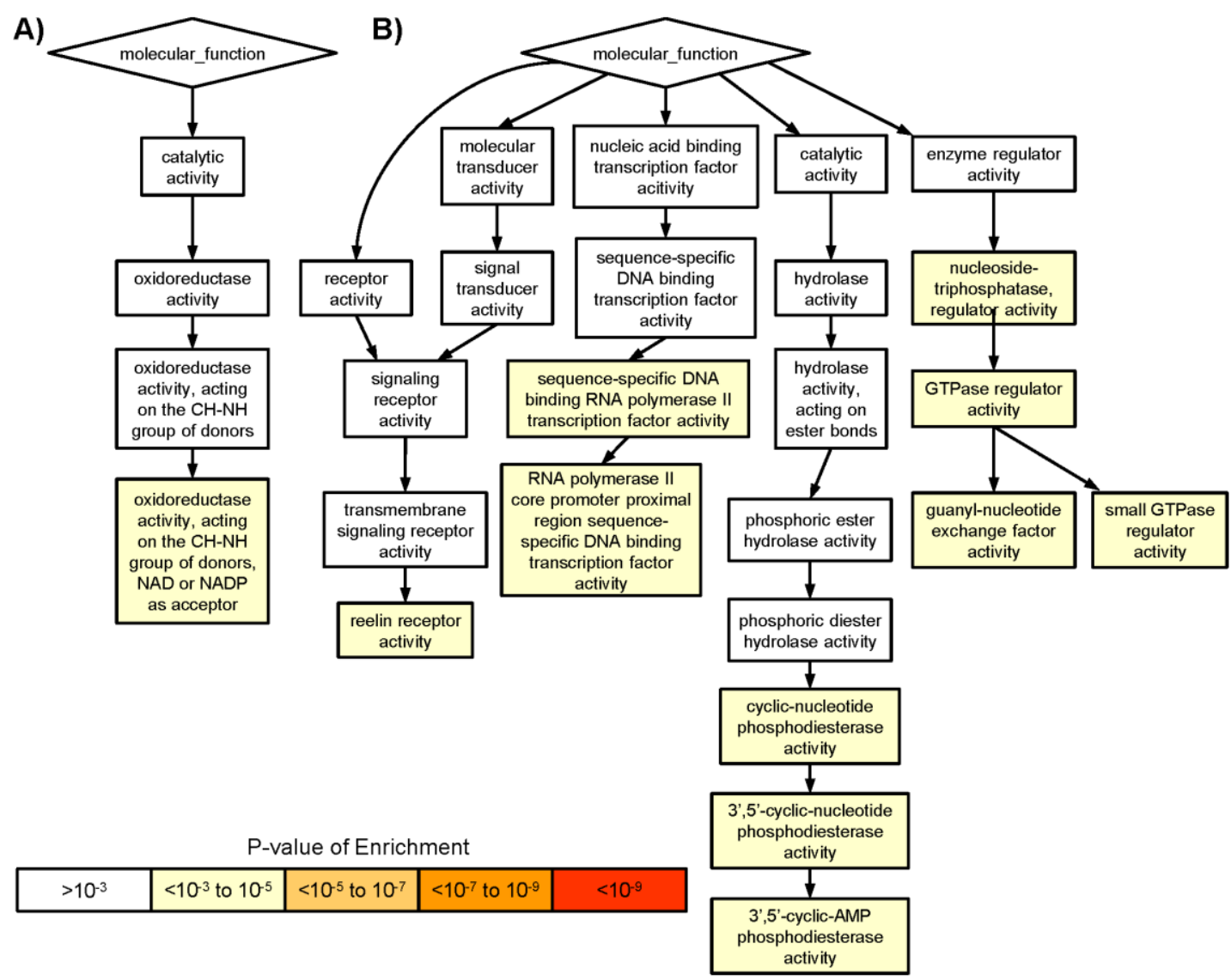

\section{Online Figure 5.IX}

Gene ontology clustering architecture of microarray expression with and without reversal of shear stress direction. Clustering of over-represented molecular functions for the 500 top transcripts as ranked by $p$ value was performed. A) Visually depicts the changes seen with non-reversed increase in shear stress compared to control after 6 hours post-"FAL". Only one significant molecular function is over-represented B) Visually depicts the changes seen with an increase in shear stress plus directional reversal compared to control after 6 hours post-"FAL". Numerous pathways are over-represented. Of particular interest, cAMP PDE activity suggests involvement of PKA and Akt signaling, while the increased activity in small GTPases suggests involvement of cytoskeletal reorganization. Degree of enrichment and genes involved for each analysis are outline in online table III. 


\section{Online Table 5.I}

Online Table 5.I. All differentially regulated genes between reversed (increased shear stress) versus control (steady shear stress) conditions with FDR<0.10

\begin{tabular}{|c|c|c|c|c|c|}
\hline Gene Name & Entrez ID & Symbol & $\log _{2} F C$ & $\begin{array}{l} \pm 95 \% \\
\text { C.I. }\end{array}$ & FDR \\
\hline \multicolumn{6}{|l|}{ A. Upregulated Genes } \\
\hline \multirow{3}{*}{$\begin{array}{l}\text { neutrophil cytosolic factor } 2 \\
\text { semaphorin } 7 \text { A, GPI membrane anchor (John Milton Hagen } \\
\text { blood group) } \\
\text { thrombomodulin }\end{array}$} & 4688 & NCF2 & 1.157 & 0.293 & $7.72 \mathrm{E}-03$ \\
\hline & 8482 & SEMA7A & 1.133 & 0.221 & $1.96 \mathrm{E}-03$ \\
\hline & 7056 & THBD & 1.002 & 0.127 & $1.91 \mathrm{E}-04$ \\
\hline 1-acylglycerol-3-phosphate O-acyltransferase 9 & 84803 & AGPAT9 & 0.971 & 0.184 & $1.96 \mathrm{E}-03$ \\
\hline parathyroid hormone-like hormone & 5744 & PTHLH & 0.914 & 0.216 & $6.37 \mathrm{E}-03$ \\
\hline chemokine (C-C motif) ligand 20 & 6364 & CCL20 & 0.870 & 0.353 & 4.73E-02 \\
\hline chemokine-like receptor 1 & 1240 & CMKLR1 & 0.860 & 0.292 & $2.58 \mathrm{E}-02$ \\
\hline ADAM metallopeptidase with thrombospondin type 1 motif, 4 & 9507 & ADAMTS4 & 0.836 & 0.204 & $6.37 \mathrm{E}-03$ \\
\hline ADAM metallopeptidase with thrombospondin type 1 motif, 9 & 56999 & ADAMTS9 & 0.820 & 0.217 & $9.70 \mathrm{E}-03$ \\
\hline serpin peptidase inhibitor, clade B (ovalbumin), member 2 & 5055 & SERPINB2 & 0.810 & 0.356 & $6.22 \mathrm{E}-02$ \\
\hline RasGEF domain family, member $1 \mathrm{~B}$ & 153020 & RASGEF1B & 0.803 & 0.372 & 7.46E-02 \\
\hline stanniocalcin 1 & 6781 & STC1 & 0.765 & 0.306 & 4.43E-02 \\
\hline nephroblastoma overexpressed & 4856 & NOV & 0.756 & 0.129 & $1.40 \mathrm{E}-03$ \\
\hline kelch repeat and BTB (POZ) domain containing 8 & 84541 & KBTBD8 & 0.737 & 0.114 & 7.39E-04 \\
\hline KIT ligand & 4254 & KITLG & 0.718 & 0.205 & $1.12 \mathrm{E}-02$ \\
\hline fermitin family member 3 & 83706 & FERMT3 & 0.695 & 0.316 & 7.04E-02 \\
\hline diacylglycerol lipase, alpha & 747 & DAGLA & 0.682 & 0.198 & $1.21 \mathrm{E}-02$ \\
\hline $\begin{array}{l}\text { sema domain, immunoglobulin domain (Ig), transmembrane } \\
\text { domain (TM) and short cytoplasmic domain, (semaphorin) } \\
\text { 4B }\end{array}$ & 10509 & SEMA4B & 0.679 & 0.125 & $1.96 \mathrm{E}-03$ \\
\hline RCAN family member 3 & 11123 & RCAN3 & 0.675 & 0.338 & $9.89 \mathrm{E}-02$ \\
\hline ras homolog family member $F$ (in filopodia) & 54509 & RHOF & 0.671 & 0.240 & $3.10 \mathrm{E}-02$ \\
\hline insulin induced gene 1 & 3638 & INSIG1 & 0.660 & 0.164 & $7.23 \mathrm{E}-03$ \\
\hline heparanase & 10855 & HPSE & 0.635 & 0.255 & $4.50 \mathrm{E}-02$ \\
\hline leucine rich repeat (in FLII) interacting protein 1 & 9208 & LRRFIP1 & 0.629 & 0.267 & $5.57 \mathrm{E}-02$ \\
\hline ADAM metallopeptidase with thrombospondin type 1 motif, 1 & 9510 & ADAMTS1 & 0.629 & 0.265 & $5.51 \mathrm{E}-02$ \\
\hline dual specificity phosphatase 5 & 1847 & DUSP5 & 0.616 & 0.139 & $5.04 \mathrm{E}-03$ \\
\hline angiopoietin-like 4 & 51129 & ANGPTL4 & 0.610 & 0.276 & $6.94 \mathrm{E}-02$ \\
\hline paraneoplastic Ma antigen 2 & 10687 & PNMA2 & 0.603 & 0.255 & $5.54 \mathrm{E}-02$ \\
\hline carbonic anhydrase XIII & 377677 & CA13 & 0.601 & 0.213 & 3.10E-02 \\
\hline dedicator of cytokinesis 11 & 139818 & DOCK11 & 0.585 & 0.223 & 3.69E-02 \\
\hline G protein-coupled receptor, family C, group 5 , member A & 9052 & GPRC5A & 0.583 & 0.253 & $5.95 \mathrm{E}-02$ \\
\hline plasminogen activator, urokinase receptor & 5329 & PLAUR & 0.583 & 0.147 & $7.72 \mathrm{E}-03$ \\
\hline FH2 domain containing 1 & 85462 & FHDC1 & 0.568 & 0.138 & $6.37 \mathrm{E}-03$ \\
\hline $\begin{array}{l}\text { 5-hydroxytryptamine (serotonin) receptor 1D, G protein- } \\
\text { coupled }\end{array}$ & 3352 & HTR1D & 0.563 & 0.271 & 8.71E-02 \\
\hline $\begin{array}{l}\text { G protein-coupled receptor } 37 \text { (endothelin receptor type B- } \\
\text { like) }\end{array}$ & 2861 & GPR37 & 0.562 & 0.241 & $5.71 \mathrm{E}-02$ \\
\hline $\begin{array}{l}\text { very low density lipoprotein receptor } \\
\text { grainyhead-like } 1 \text { (Drosophila) }\end{array}$ & $\begin{array}{l}7436 \\
29841\end{array}$ & $\begin{array}{l}\text { VLDLR } \\
\text { GRHL1 }\end{array}$ & $\begin{array}{l}0.547 \\
0.547\end{array}$ & $\begin{array}{l}0.261 \\
0.129\end{array}$ & $\begin{array}{l}8.48 \mathrm{E}-02 \\
6.37 \mathrm{E}-03\end{array}$ \\
\hline $\begin{array}{l}\text { UDP-N-acetyl-alpha-D-galactosamine:polypeptide } \\
\text { acetylgalactosaminyltransferase } 12 \text { (GalNAc-T12) }\end{array}$ & 79695 & GALNT12 & 0.545 & 0.260 & $8.48 \mathrm{E}-02$ \\
\hline \multirow{4}{*}{$\begin{array}{l}\text { neurotensin receptor } 1 \text { (high affinity) } \\
\text { Rap guanine nucleotide exchange factor (GEF) } 5 \\
\text { desert hedgehog } \\
\text { pleckstrin homology domain containing, family G (with } \\
\text { RhoGef domain) member } 1\end{array}$} & 4923 & NTSR1 & 0.544 & 0.130 & $6.37 \mathrm{E}-03$ \\
\hline & 9771 & RAPGEF5 & 0.541 & 0.139 & 7.72E-03 \\
\hline & 50846 & $\mathrm{DHH}$ & 0.531 & 0.170 & 1.97E-02 \\
\hline & 57480 & PLEKHG1 & 0.523 & 0.117 & 4.79E-03 \\
\hline fibroblast growth factor 18 & 8817 & FGF18 & 0.523 & 0.185 & $3.10 \mathrm{E}-02$ \\
\hline $\begin{array}{l}\text { Ras association (RalGDS/AF-6) and pleckstrin homology } \\
\text { domains } 1\end{array}$ & 65059 & RAPH1 & 0.522 & 0.149 & $1.14 \mathrm{E}-02$ \\
\hline \multirow{2}{*}{ fibroblast growth factor 16} & 8823 & FGF16 & 0.520 & 0.200 & 3.77E-02 \\
\hline & 51294 & PCDH12 & 0.508 & 0.163 & 1.97E-02 \\
\hline \multirow{2}{*}{$\begin{array}{l}\text { solute carrier family } 35 \text {, member E4 } \\
\text { carbohydrate (chondroitin } 6 \text { ) sulfotransferase } 3\end{array}$} & 339665 & SLC35E4 & 0.506 & 0.144 & $1.12 \mathrm{E}-02$ \\
\hline & 9469 & CHST3 & 0.503 & 0.213 & 5.57E-02 \\
\hline $\begin{array}{c}\text { natriuretic peptide receptor } \mathrm{A} / \text { guanylate cyclase } \mathrm{A} \\
\text { (atrionatriuretic peptide receptor } \mathrm{A} \text { ) }\end{array}$ & 4881 & NPR1 & 0.503 & 0.188 & 3.37E-02 \\
\hline \multirow{2}{*}{$\begin{array}{l}\text { chromosome } 3 \text { open reading frame } 55 \\
\text { TSC22 domain family, member } 3\end{array}$} & 152078 & C3orf55 & 0.498 & 0.141 & $1.12 \mathrm{E}-02$ \\
\hline & 1831 & TSC22D3 & 0.489 & 0.171 & 2.93E-02 \\
\hline family with sequence similarity 134 , member $B$ & & & 0.487 & 0.181 & 3.23E-02 \\
\hline TBC1 domain family, member 2 & 55357 & TBC1D2 & 0.485 & 0.161 & $2.28 \mathrm{E}-02$ \\
\hline solute carrier organic anion transporter family, member $4 \mathrm{C} 1$ & 353189 & SLCO4C1 & 0.485 & 0.193 & $4.28 \mathrm{E}-02$ \\
\hline small nucleolar RNA, H/ACA box 1 & 677792 & SNORA1 & 0.482 & 0.237 & $9.51 \mathrm{E}-02$ \\
\hline LIM domain 7 & 4008 & LMO7 & 0.477 & 0.171 & $3.10 \mathrm{E}-02$ \\
\hline ADAM metallopeptidase domain 19 & 8728 & ADAM19 & 0.476 & 0.139 & $1.21 \mathrm{E}-02$ \\
\hline nitric oxide synthase 3 (endothelial cell) & 4846 & NOS3 & 0.474 & 0.131 & $1.08 \mathrm{E}-02$ \\
\hline Kruppel-like factor 4 (gut) & 9314 & KLF4 & 0.473 & 0.129 & $1.02 \mathrm{E}-02$ \\
\hline small cell adhesion glycoprotein & 57228 & SMAGP & 0.469 & 0.113 & $6.37 \mathrm{E}-03$ \\
\hline StAR-related lipid transfer (START) domain containing 8 & 9754 & STARD8 & 0.465 & 0.126 & $1.02 \mathrm{E}-02$ \\
\hline interleukin 11 & 3589 & IL11 & 0.456 & 0.153 & $2.48 \mathrm{E}-02$ \\
\hline
\end{tabular}




\begin{tabular}{|c|c|c|c|c|c|}
\hline $\begin{array}{l}\text { cytochrome b5 reductase } 2 \\
\text { CASK interacting protein } 2\end{array}$ & $\begin{array}{l}51700 \\
57513\end{array}$ & $\begin{array}{l}\text { CYB5R2 } \\
\text { CASKIN2 }\end{array}$ & $\begin{array}{l}0.456 \\
0.455\end{array}$ & 0.221 & $\begin{array}{l}8.98 \mathrm{E}-02 \\
1.12 \mathrm{E}-02\end{array}$ \\
\hline $\begin{array}{l}\text { CASK interacting protein } 2 \\
\text { solute carrier family } 7 \text { (amino acid transporter light chain, L }\end{array}$ & 57513 & CASKIN2 & 0.455 & 0.128 & \\
\hline system), member 5 & 8140 & SLC7A5 & 0.454 & 0.215 & 8.17E-02 \\
\hline $\begin{array}{l}\text { GTP cyclohydrolase } 1 \\
\text { ephrin-B1 }\end{array}$ & $\begin{array}{l}2643 \\
1947\end{array}$ & $\begin{array}{l}\text { GCH1 } \\
\text { EFNB1 }\end{array}$ & $\begin{array}{l}0.454 \\
0.453\end{array}$ & $\begin{array}{l}0.224 \\
0.178\end{array}$ & $\begin{array}{l}9.51 \mathrm{E}-02 \\
4.15 \mathrm{E}-02\end{array}$ \\
\hline $\begin{array}{l}\text { olfactory receptor, family } 2 \text {, subfamily } A, \text { member } 9 \\
\text { pseudogene }\end{array}$ & 441295 & OR2A9P & 0.444 & 0.207 & 7.57E-02 \\
\hline odz, odd Oz/ten-m homolog 3 (Drosophila) & 55714 & ODZ3 & 0.441 & 0.143 & 2.02E-02 \\
\hline platelet-derived growth factor alpha polypeptide & 5154 & PDGFA & 0.441 & 0.162 & $3.22 \mathrm{E}-02$ \\
\hline myelin protein zero-like 3 & 196264 & MPZL3 & 0.436 & 0.157 & 3.14E-02 \\
\hline OTU domain, ubiquitin aldehyde binding 2 & 78990 & OTUB2 & 0.434 & 0.140 & $2.02 \mathrm{E}-02$ \\
\hline claudin 1 & 9076 & CLDN1 & 0.433 & 0.217 & $9.84 \mathrm{E}-02$ \\
\hline versican & 1462 & VCAN & 0.431 & 0.195 & $6.89 \mathrm{E}-02$ \\
\hline sprouty homolog 4 (Drosophila) & 81848 & SPRY4 & 0.430 & 0.200 & $7.47 \mathrm{E}-02$ \\
\hline tumor necrosis factor receptor superfamily, member 9 & 3604 & TNFRSF9 & 0.427 & 0.133 & $1.74 \mathrm{E}-02$ \\
\hline KIAA1522 & 57648 & KIAA1522 & 0.426 & 0.116 & $1.02 \mathrm{E}-02$ \\
\hline sonic hedgehog & 6469 & $\mathrm{SHH}$ & 0.424 & 0.177 & $5.45 \mathrm{E}-02$ \\
\hline cAMP responsive element binding protein 5 & 9586 & CREB5 & 0.424 & 0.134 & $1.90 \mathrm{E}-02$ \\
\hline meningioma (disrupted in balanced translocation) 1 & 4330 & MN1 & 0.423 & 0.139 & $2.22 \mathrm{E}-02$ \\
\hline potassium channel, subfamily K, member 1 & 3775 & KCNK1 & 0.417 & 0.144 & $2.72 \mathrm{E}-02$ \\
\hline epithelial mitogen homolog (mouse) & 255324 & EPGN & 0.411 & 0.163 & $4.31 \mathrm{E}-02$ \\
\hline BCL2-antagonist/killer 1 & 578 & BAK1 & 0.408 & 0.186 & $7.04 \mathrm{E}-02$ \\
\hline protein tyrosine phosphatase, receptor type, $\mathrm{J}$ & 5795 & PTPRJ & 0.407 & 0.137 & $2.48 \mathrm{E}-02$ \\
\hline LY6/PLAUR domain containing 5 & 284348 & LYPD5 & 0.406 & 0.149 & $3.21 \mathrm{E}-02$ \\
\hline Kruppel-like factor 8 & 11279 & KLF8 & 0.404 & 0.197 & $9.01 \mathrm{E}-02$ \\
\hline $\begin{array}{l}\text { methylenetetrahydrofolate dehydrogenase } \\
\text { dependent) } 2 \text {, methenyltetrahydrofolate cyclohydrolase }\end{array}$ & 10797 & MTHFD2 & 0.404 & 0.153 & $3.51 \mathrm{E}-02$ \\
\hline basic, immunoglobulin-like variable motif containing & 54841 & BIVM & 0.403 & 0.174 & $5.88 \mathrm{E}-02$ \\
\hline $\begin{array}{l}\text { UDP-GIcNAc:betaGal } \\
\text { acetylglucosaminyltransferase } 2 \\
\text { beta-1,3-N- }\end{array}$ & 10678 & B3GNT2 & 0.400 & 0.152 & $3.56 \mathrm{E}-02$ \\
\hline zinc finger protein 467 & 168544 & ZNF467 & 0.397 & 0.156 & 4.13E-02 \\
\hline delta-like 1 (Drosophila) & 28514 & DLL1 & 0.396 & 0.156 & 4.15E-02 \\
\hline cell division cycle 6 homolog (S. cerevisiae) & 990 & CDC6 & 0.393 & 0.143 & 3.14E- $\mathrm{C}$ \\
\hline interleukin 1 receptor, type I & 3554 & IL1R1 & 0.392 & 0.171 & $6.01 \mathrm{E}-\mathrm{C}$ \\
\hline tensin 1 & 7145 & TNS1 & 0.391 & 0.152 & 4.05E- $-\mathrm{C}$ \\
\hline delta/notch-like EGF repeat containing & 92737 & DNER & 0.388 & 0.187 & $8.76 \mathrm{E}-\mathrm{C}$ \\
\hline mixed lineage kinase domain-like & 197259 & MLKL & 0.385 & 0.182 & $8.22 \mathrm{E}-\mathrm{C}$ \\
\hline small nucleolar RNA, C/D box 50B & 692088 & SNORD50B & 0.383 & 0.189 & $9.54 \mathrm{E}-\mathrm{C}$ \\
\hline gasdermin C & 56169 & GSDMC & 0.383 & 0.178 & 7.47E-C \\
\hline chemokine (C-C motif) receptor-like 2 & 9034 & CCRL2 & 0.383 & 0.170 & $6.43 \mathrm{E}-\mathrm{C}$ \\
\hline plasminogen activator, urokinase & 5328 & PLAU & 0.381 & 0.160 & $5.51 \mathrm{E}-\mathrm{C}$ \\
\hline Kruppel-like factor 11 & 8462 & KLF11 & 0.380 & 0.107 & $1.12 \mathrm{E}-\mathrm{C}$ \\
\hline coagulation factor II (thrombin) receptor-like 3 & 9002 & F2RL3 & 0.377 & 0.135 & 3.10E-C \\
\hline Epstein-Barr virus induced 3 & 10148 & $\mathrm{EBI} 3$ & 0.376 & 0.169 & $6.75 \mathrm{E}-\mathrm{C}$ \\
\hline microtubule-associated protein $1 \mathrm{~A}$ & 4130 & MAP1A & 0.374 & 0.135 & 3.14E-C \\
\hline DnaJ (Hsp40) homolog, subfamily B, member 9 & 4189 & DNAJB9 & 0.374 & 0.131 & 2.93E-C \\
\hline zinc finger protein 589 & 51385 & ZNF589 & 0.373 & 0.144 & $3.86 \mathrm{E}-\mathrm{C}$ \\
\hline zinc finger protein 643 & 65243 & ZNF643 & 0.373 & 0.178 & $8.45 \mathrm{E}-\mathrm{C}$ \\
\hline ankyrin repeat domain 20 family, member $\mathrm{A} 3$ & 441425 & ANKRD20A3 & 0.371 & 0.159 & 5.66E- \\
\hline $\begin{array}{l}\text { low density lipoprotein receptor-related protein } 8 \text {, } \\
\text { apolipoprotein e receptor }\end{array}$ & 7804 & LRP8 & 0.369 & 0.164 & $6.59 \mathrm{E}-02$ \\
\hline chromosome 14 open reading frame 43 & 91748 & C14orf43 & 0.368 & 0.175 & 8.43E-02 \\
\hline pantothenate kinase 2 & 80025 & PANK2 & 0.368 & 0.171 & $\begin{array}{l}7.47 \mathrm{E}-\mathrm{C} \\
3.14 \mathrm{E}-\mathrm{C}\end{array}$ \\
\hline KIAA1199 & 57214 & KIAA1199 & 0.367 & 0.132 & $3.14 \mathrm{E}-\mathrm{C}$ \\
\hline proline rich 5 like & 79899 & PRR5L & 0.366 & 0.139 & 3.56E- $-\mathrm{C}$ \\
\hline ganglioside induced differentiation associated protein 1 & 54332 & GDAP1 & 0.365 & 0.166 & $7.04 \mathrm{E}-\mathrm{C}$ \\
\hline EPH receptor A4 & 2043 & EPHA4 & 0.364 & 0.134 & 3.22E-C \\
\hline tribbles homolog 1 (Drosophila) & 10221 & TRIB1 & 0.363 & 0.131 & $3.14 \mathrm{E}-\mathrm{C}$ \\
\hline fibroblast growth factor 12 & 2257 & FGF12 & 0.360 & 0.152 & $5.51 \mathrm{E}-\mathrm{C}$ \\
\hline HEG homolog 1 (zebrafish) & 57493 & HEG1 & 0.359 & 0.125 & $2.90 \mathrm{E}-\mathrm{C}$ \\
\hline sphingomyelin synthase 2 & 166929 & SGMS2 & 0.359 & 0.100 & $1.12 \mathrm{E}-\mathrm{C}$ \\
\hline spectrin repeat containing, nuclear envelope family member 3 & 161176 & SYNE3 & 0.358 & 0.139 & $3.92 \mathrm{E}-\mathrm{C}$ \\
\hline FERM domain containing 3 & 257019 & FRMD3 & 0.358 & 0.126 & 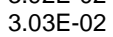 \\
\hline CXXC finger protein 5 & 51523 & CXXC5 & 0.357 & 0.137 & 3.70E- $\mathrm{C}$ \\
\hline NEDD4 binding protein 2 & 55728 & N4BP2 & 0.356 & 0.161 & $6.89 \mathrm{E}-\mathrm{C}$ \\
\hline chromosome $\mathrm{X}$ open reading frame 23 & 256643 & CXorf23 & 0.354 & 0.160 & $6.89 \mathrm{E}-\mathrm{C}$ \\
\hline thromboxane $\mathrm{A} 2$ receptor & 6915 & TBXA2R & 0.354 & 0.143 & 4.57E- $\mathrm{C}$ \\
\hline Kruppel-like factor 13 & 51621 & KLF13 & 0.354 & 0.159 & 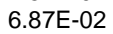 \\
\hline zinc finger, MIZ-type containing 1 & 57178 & ZMIZ1 & 0.353 & 0.113 & $1.97 \mathrm{E}-\mathrm{C}$ \\
\hline chromosome 15 open reading frame 26 & 161502 & C15orf26 & 0.352 & 0.126 & 3.10E- $-\mathrm{C}$ \\
\hline laminin, alpha 5 & 3911 & $\begin{array}{l}\text { LAMA5 } \\
\text { LAM }\end{array}$ & 0.352 & 0.131 & 3.36E- $\mathrm{C}$ \\
\hline inositol 1,4,5-trisphosphate receptor, type 3 & 3710 & ITPR3 & 0.351 & 0.110 & $1.81 \mathrm{E}-\mathrm{C}$ \\
\hline endonuclease domain containing 1 & 23052 & ENDOD1 & 0.349 & 0.151 & $5.89 \mathrm{E}-\mathrm{C}$ \\
\hline sushi domain containing 1 & 64420 & SUSD1 & 0.346 & 0.144 & $5.22 \mathrm{E}-\mathrm{C}$ \\
\hline heat shock $70 \mathrm{kD}$ protein $12 \mathrm{~B}$ & 116835 & HSPA12B & 0.346 & 0.139 & $4.50 \mathrm{E}-\mathrm{C}$ \\
\hline Niemann-Pick disease, type $\mathrm{C} 1$ & 4864 & NPC1 & 0.344 & 0.137 & $4.26 \mathrm{E}-\mathrm{C}$ \\
\hline FIC domain containing & 11153 & FICD & 0.344 & 0.150 & $6.01 \mathrm{E}-($ \\
\hline sprouty-related, EVH1 domain containing 1 & 161742 & SPRED1 & 0.343 & 0.169 & $9.61 \mathrm{E}-($ \\
\hline fibroblast growth factor receptor 3 & 2261 & FGFR3 & 0.340 & 0.145 & $5.66 \mathrm{E}-\mathrm{C}$ \\
\hline
\end{tabular}




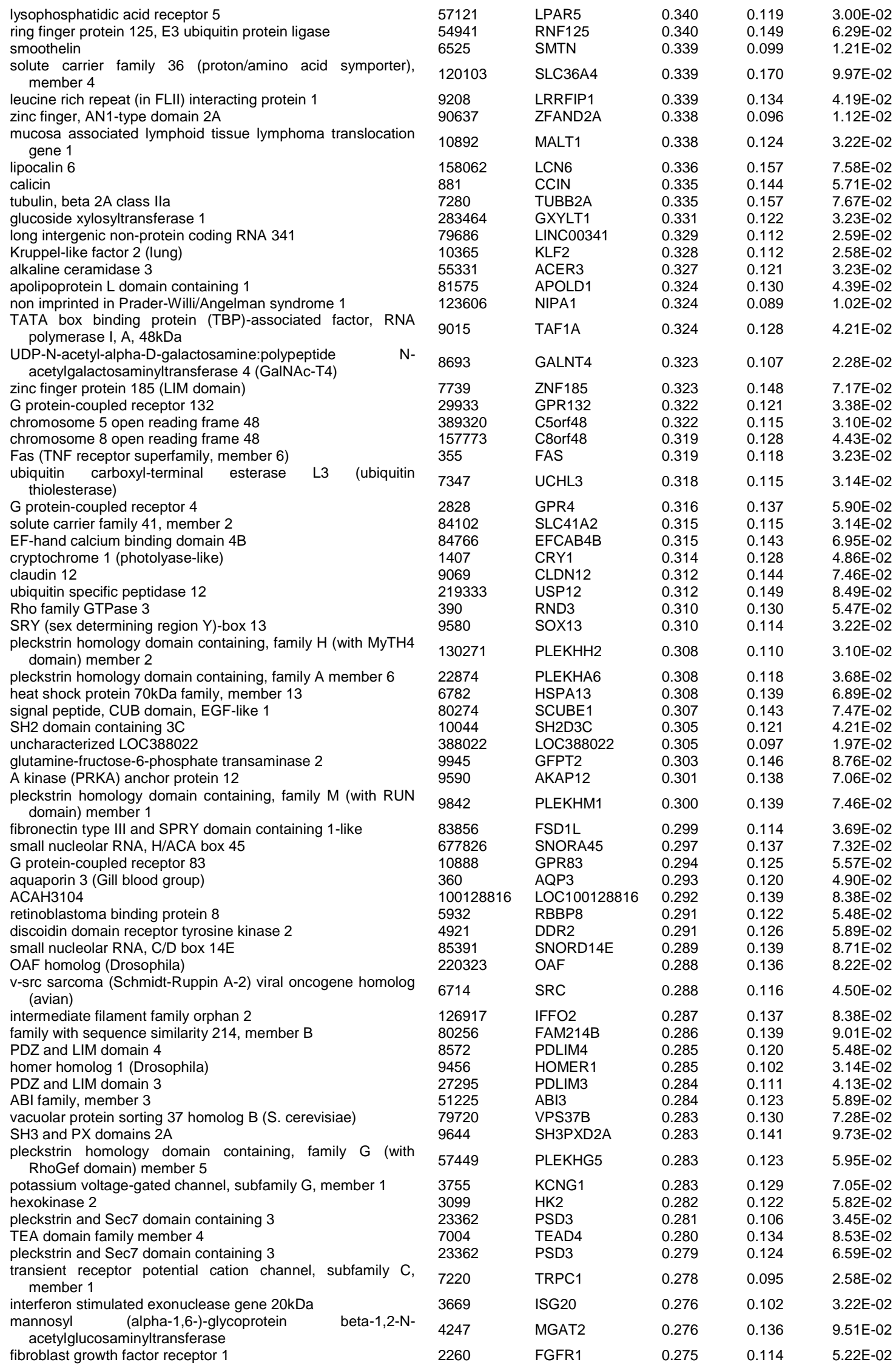


lysophosphatidylcholine acyltransferase 4

cardiotrophin-like cytokine factor 1

thrombospondin, type I, domain containing 4

golgi SNAP receptor complex member 2

thrombospondin, type I, domain containing 1

family with sequence similarity 126 , member A

protein kinase (cAMP-dependent, catalytic) inhibitor gamma

actin filament associated protein 1-like 1

mall nucleolar RNA, C/D box 54

v-ets erythroblastosis virus E26 oncogene homolog 1 (avian)

tubulin, alpha $4 \mathrm{a}$

G protein-coupled receptor 17

JAZF zinc finger 1

cyclin D3

phosphodiesterase 2A, cGMP-stimulated

myocyte enhancer factor $2 A$

ATP-binding cassette, sub-family G (WHITE), member 2

REST corepressor 1

armadillo repeat containing 7

STE20-like kinase

dual specificity phosphatase 1

chromodomain helicase DNA binding protein 9

UDP-GlcNAc:betaGal

acetylglucosaminyltransferase 5

nuclear receptor subfamily 4 , group A, member 1

prenyl (decaprenyl) diphosphate synthase, subunit 1

ATPase, $\mathrm{Ca}++$ transporting, plasma membrane 4

family with sequence similarity 40 , member $B$

lysophosphatidylcholine acyltransferase 1

GrpE-like 1, mitochondrial (E. coli)

ST3 beta-galactoside alpha-2,3-sialyltransferase 1

epithelial membrane protein 3

dual specificity phosphatase 7

human immunodeficiency virus type I enhancer binding protein 3

E74-like factor 4 (ets domain transcription factor)

aryl hydrocarbon receptor nuclear translocator-like

mannosyl (alpha-1,3-)-glycoprotein beta-1,4-Nacetylglucosaminyltransferase, isozyme A

Ras interacting protein 1

fem-1 homolog b (C. elegans)

$\mathrm{N}$-myc downstream regulated

kinesin family member $\mathrm{C} 1$

ATPase type $13 \mathrm{~A} 3$

solute carrier family 33 (acetyl-CoA transporter), member 1

MAX dimerization protein 1

Ras-like without CAAX 1

family with sequence similarity 65 , member $A$

pleckstrin homology domain containing, family M (with RUN

domain) member 1 pseudogene

suppressor of cytokine signaling 1

ring finger protein 149

collagen, type XIII, alpha 1

$G$ protein-coupled receptor kinase 5

signal-induced proliferation-associated 1 like 3

fibrillin 1

RAS $p 21$ protein activator 4

Fc receptor-like A

potassium voltage-gated channel, Shaw-related subfamily, member 4

ATPase, $\mathrm{H}+$ transporting, lysosomal $34 \mathrm{kDa}, \mathrm{V} 1$ subunit D

ZNF625-ZNF20 readthrough

B-cell CLL/lymphoma 9-like

inositol(myo)-1(or 4)-monophosphatase 1

zinc finger and BTB domain containing 43

peripheral myelin protein 22

protein kinase-like protein SgK196

asparagine-linked glycosylation 5, dolichyl-phosphate betaglucosyltransferase homolog (S. cerevisiae)

Bardet-Biedl syndrome 7

forkhead box N2

KIAA1244

interleukin 20 receptor beta

tyrosine 3-monooxygenase/tryptophan 5-monooxygenase activation protein, eta polypeptide

patatin-like phospholipase domain containing 8

transcription elongation factor B (SIII), polypeptide 1 (15kDa, elongin C)

Meis homeobox 3 pseudogene 1

cytohesin 1

\begin{tabular}{|c|c|c|c|c|}
\hline 254531 & LPCAT4 & 0.274 & 0.135 & $9.44 \mathrm{E}-02$ \\
\hline 23529 & CLCF1 & 0.271 & 0.125 & $7.25 \mathrm{E}-02$ \\
\hline 79875 & THSD4 & 0.271 & 0.120 & $6.43 \mathrm{E}-02$ \\
\hline 9570 & GOSR2 & 0.268 & 0.098 & 3.17E-02 \\
\hline 55901 & THSD1 & 0.267 & 0.112 & $5.51 \mathrm{E}-02$ \\
\hline 84668 & FAM126A & 0.266 & 0.114 & $5.78 \mathrm{E}-02$ \\
\hline 11142 & PKIG & 0.266 & 0.116 & $6.01 \mathrm{E}-02$ \\
\hline 134265 & AFAP1L1 & 0.265 & 0.117 & $6.43 \mathrm{E}-02$ \\
\hline 26795 & SNORD54 & 0.263 & 0.119 & 6.89E-02 \\
\hline 2113 & ETS1 & 0.262 & 0.132 & 9.97E-02 \\
\hline 7277 & TUBA4A & 0.262 & 0.119 & 6.97E-02 \\
\hline 2840 & GPR17 & 0.260 & 0.110 & 5.57E-02 \\
\hline 221895 & JAZF1 & 0.258 & 0.128 & 9.73E-02 \\
\hline 896 & CCND3 & 0.257 & 0.110 & $5.78 \mathrm{E}-02$ \\
\hline 5138 & PDE2A & 0.255 & 0.110 & $5.88 \mathrm{E}-02$ \\
\hline 4205 & MEF2A & 0.253 & 0.113 & 6.78E-02 \\
\hline 9429 & ABCG2 & 0.252 & 0.110 & 5.94E-02 \\
\hline 23186 & RCOR1 & 0.252 & 0.106 & $5.51 \mathrm{E}-02$ \\
\hline 79637 & ARMC7 & 0.250 & 0.125 & 9.94E-02 \\
\hline 9748 & SLK & 0.250 & 0.093 & 3.23E-02 \\
\hline 1843 & DUSP1 & 0.250 & 0.103 & 5.19E-02 \\
\hline 80205 & CHD9 & 0.250 & 0.124 & $9.62 \mathrm{E}-02$ \\
\hline 84002 & B3GNT5 & 0.249 & 0.114 & 7.06E-02 \\
\hline 3164 & NR4A1 & 0.247 & 0.101 & $4.86 \mathrm{E}-02$ \\
\hline 23590 & PDSS1 & 0.246 & 0.118 & 8.53E-02 \\
\hline 493 & ATP2B4 & 0.246 & 0.122 & $9.63 \mathrm{E}-02$ \\
\hline 57464 & FAM40B & 0.243 & 0.113 & 7.47E-02 \\
\hline 79888 & LPCAT1 & 0.242 & 0.104 & $5.80 \mathrm{E}-02$ \\
\hline 80273 & GRPEL1 & 0.242 & 0.101 & $5.48 \mathrm{E}-02$ \\
\hline 6482 & ST3GAL1 & 0.241 & 0.105 & $5.98 \mathrm{E}-02$ \\
\hline 2014 & EMP3 & 0.241 & 0.116 & $8.78 \mathrm{E}-02$ \\
\hline 1849 & DUSP7 & 0.241 & 0.096 & 4.37E-02 \\
\hline 59269 & HIVEP3 & 0.237 & 0.101 & 5.57E-02 \\
\hline $\begin{array}{l}2000 \\
406\end{array}$ & $\begin{array}{l}\text { ELF4 } \\
\text { ARNTL }\end{array}$ & $\begin{array}{l}0.235 \\
0.234\end{array}$ & $\begin{array}{l}0.111 \\
0.100\end{array}$ & $\begin{array}{l}\text { 8.23E-02 } \\
5.75 \mathrm{E}-02\end{array}$ \\
\hline 11320 & MGAT4A & 0.231 & 0.104 & $6.89 \mathrm{E}-02$ \\
\hline 54922 & RASIP1 & 0.231 & 0.115 & 9.77E-02 \\
\hline 10116 & FEM1B & 0.228 & 0.109 & 8.31E-02 \\
\hline 10397 & NDRG1 & 0.228 & 0.111 & 8.96E-02 \\
\hline 3833 & KIFC1 & 0.226 & 0.102 & $6.88 \mathrm{E}-02$ \\
\hline 79572 & ATP13A3 & 0.226 & 0.095 & $5.51 \mathrm{E}-02$ \\
\hline 9197 & SLC33A1 & 0.226 & 0.112 & $9.73 \mathrm{E}-02$ \\
\hline 4084 & MXD1 & 0.225 & 0.108 & 8.49E-02 \\
\hline 6016 & RIT1 & 0.225 & 0.095 & $5.51 \mathrm{E}-02$ \\
\hline 79567 & FAM65A & 0.224 & 0.107 & $8.38 \mathrm{E}-02$ \\
\hline 440456 & PLEKHM1P & 0.223 & 0.096 & 5.82E-02 \\
\hline 8651 & socs1 & 0.223 & 0.099 & $6.53 \mathrm{E}-02$ \\
\hline 284996 & RNF149 & 0.223 & 0.092 & $5.20 \mathrm{E}-02$ \\
\hline 1305 & COL13A1 & 0.220 & 0.110 & 9.77E-02 \\
\hline 2869 & GRK5 & 0.220 & 0.100 & 7.04E-02 \\
\hline 23094 & SIPA1L3 & 0.220 & 0.108 & $9.31 \mathrm{E}-02$ \\
\hline 2200 & FBN1 & 0.219 & 0.100 & 7.06E-02 \\
\hline 10156 & RASA4 & 0.218 & 0.100 & 7.10E-02 \\
\hline 84824 & FCRLA & 0.217 & 0.108 & $9.61 \mathrm{E}-02$ \\
\hline 3749 & KCNC4 & 0.215 & 0.092 & $5.66 \mathrm{E}-02$ \\
\hline 51382 & ATP6V1D & 0.213 & 0.103 & $8.78 \mathrm{E}-02$ \\
\hline 100529855 & ZNF625-ZNF20 & 0.213 & 0.107 & $9.94 \mathrm{E}-02$ \\
\hline 283149 & BCL9L & 0.212 & 0.098 & 7.46E-02 \\
\hline 3612 & IMPA1 & 0.211 & 0.104 & $9.45 \mathrm{E}-02$ \\
\hline 23099 & ZBTB43 & 0.210 & 0.102 & 8.90E-02 \\
\hline 5376 & PMP22 & 0.209 & 0.099 & $8.38 \mathrm{E}-02$ \\
\hline 84197 & SGK196 & 0.208 & 0.104 & $9.89 \mathrm{E}-02$ \\
\hline 29880 & ALG5 & 0.204 & 0.096 & $7.84 \mathrm{E}-02$ \\
\hline 55212 & BBS7 & 0.203 & 0.101 & $9.61 \mathrm{E}-02$ \\
\hline 3344 & FOXN2 & 0.202 & 0.094 & 7.47E-02 \\
\hline 57221 & KIAA1244 & 0.202 & 0.098 & $9.01 \mathrm{E}-02$ \\
\hline 53833 & IL20RB & 0.201 & 0.089 & $6.43 \mathrm{E}-02$ \\
\hline 7533 & YWHAH & 0.201 & 0.092 & 7.06E-02 \\
\hline 50640 & PNPLA8 & 0.196 & 0.096 & $9.30 \mathrm{E}-02$ \\
\hline 6921 & TCEB1 & 0.196 & 0.097 & $9.75 \mathrm{E}-02$ \\
\hline 4213 & MEIS3P1 & 0.193 & 0.093 & 8.82E-02 \\
\hline 9267 & CYTH1 & 0.190 & 0.088 & 7.40E-02 \\
\hline
\end{tabular}




\begin{tabular}{|c|c|c|c|c|c|}
\hline $\begin{array}{l}\text { alpha 1,4-galactosyltransferase } \\
\text { SERTA domain containing } 2 \\
\text { family with sequence similarity } 160, \text { member A2 }\end{array}$ & $\begin{array}{l}53947 \\
9792 \\
84067\end{array}$ & $\begin{array}{l}\text { A4GALT } \\
\text { SERTAD2 } \\
\text { FAM160A2 }\end{array}$ & $\begin{array}{l}0.187 \\
0.185 \\
0.184\end{array}$ & $\begin{array}{l}0.092 \\
0.090 \\
0.092\end{array}$ & $\begin{array}{l}9.44 \mathrm{E}-02 \\
9.21 \mathrm{E}-02 \\
9.77 \mathrm{E}-02\end{array}$ \\
\hline \multicolumn{6}{|l|}{ Downregulated Genes } \\
\hline $\begin{array}{l}\text { ubiquitin D } \\
\text { ADP-ribosyltransferase } 4 \text { (Dombrock blood group) } \\
\text { microRNA } 146 \text { a } \\
\text { selectin } E \\
\text { endothelin } 1 \\
\text { retinoic acid receptor, beta } \\
\text { guanylate binding protein } 4 \\
\text { chromosome } 15 \text { open reading frame } 54 \\
\text { ets variant } 1\end{array}$ & $\begin{array}{l}10537 \\
420 \\
406938 \\
6401 \\
1906 \\
5915 \\
115361 \\
400360 \\
2115\end{array}$ & $\begin{array}{l}\text { UBD } \\
\text { ART4 } \\
\text { MIR146A } \\
\text { SELE } \\
\text { EDN1 } \\
\text { RARB } \\
\text { GBP4 } \\
\text { C15orf54 } \\
\text { ETV1 }\end{array}$ & $\begin{array}{l}-0.967 \\
-0.951 \\
-0.945 \\
-0.861 \\
-0.816 \\
-0.791 \\
-0.783 \\
-0.764 \\
-0.760\end{array}$ & $\begin{array}{l}0.478 \\
0.185 \\
0.252 \\
0.319 \\
0.231 \\
0.298 \\
0.239 \\
0.294 \\
0.205\end{array}$ & $\begin{array}{l}9.61 \mathrm{E}-02 \\
1.96 \mathrm{E}-03 \\
1.00 \mathrm{E}-02 \\
3.23 \mathrm{E}-02 \\
1.12 \mathrm{E}-02 \\
3.47 \mathrm{E}-02 \\
1.52 \mathrm{E}-02 \\
3.77 \mathrm{E}-02 \\
1.02 \mathrm{E}-02\end{array}$ \\
\hline \multirow{2}{*}{$\begin{array}{l}\text { 5-hydroxytryptamine (serotonin) receptor 2B, G protein- } \\
\text { coupled } \\
\text { multiple } \mathrm{C} 2 \text { domains, transmembrane } 1 \\
\text { guanylate binding protein } 2 \text {, interferon-inducible } \\
\text { raftlin family member } 2\end{array}$} & 3357 & HTR2B & -0.724 & 0.292 & 4.56E-02 \\
\hline & $\begin{array}{l}79772 \\
2634 \\
130132\end{array}$ & $\begin{array}{l}\text { MCTP1 } \\
\text { GBP2 } \\
\text { RFTN2 }\end{array}$ & $\begin{array}{l}-0.696 \\
-0.691 \\
-0.689\end{array}$ & $\begin{array}{l}0.201 \\
0.195 \\
0.177\end{array}$ & $\begin{array}{l}1.17 \mathrm{E}-02 \\
1.12 \mathrm{E}-02 \\
7.72 \mathrm{E}-03\end{array}$ \\
\hline $\begin{array}{l}\text { phosphatidylinositol-3,4,5-trisphosphate-dependent } \\
\text { exchange factor } 2\end{array}$ & 80243 & PREX2 & -0.661 & 0.200 & $1.52 \mathrm{E}-02$ \\
\hline $\begin{array}{l}\text { sestrin } 3 \\
\text { chemokine (C-X-C motif) receptor } 4\end{array}$ & $\begin{array}{l}143686 \\
7852\end{array}$ & $\begin{array}{l}\text { SESN3 } \\
\text { CXCR4 }\end{array}$ & $\begin{array}{l}-0.652 \\
-0.614\end{array}$ & $\begin{array}{l}0.155 \\
0.245\end{array}$ & $\begin{array}{l}\text { 6.37E-03 } \\
4.36 \mathrm{E}-02\end{array}$ \\
\hline Rho GTPase activating protein 28 & 79822 & ARHGAP28 & -0.613 & 0.200 & $2.16 \mathrm{E}-02$ \\
\hline tumor necrosis factor (ligand) superfamily, member 10 & 8743 & TNFSF10 & -0.613 & 0.219 & 3.10E-02 \\
\hline Rho GTPase activating protein 18 & 93663 & ARHGAP18 & -0.612 & 0.165 & $1.02 \mathrm{E}-02$ \\
\hline $\begin{array}{l}\text { epidermal growth factor receptor pathway substrate } 8 \\
\text { NADPH oxidase } 4\end{array}$ & $\begin{array}{l}2059 \\
50507\end{array}$ & $\begin{array}{l}\text { EPS8 } \\
\text { NOX4 }\end{array}$ & $\begin{array}{l}-0.595 \\
-0.581\end{array}$ & $\begin{array}{l}0.152 \\
0.191\end{array}$ & $\begin{array}{l}7.72 \mathrm{E}-03 \\
2.22 \mathrm{E}-02\end{array}$ \\
\hline leucine-rich repeats and immunoglobulin-like domains 3 & 121227 & LRIG3 & -0.570 & 0.223 & 4.13E-02 \\
\hline protein phosphatase 1 , regulatory subunit $16 \mathrm{~B}$ & 26051 & PPP1R16B & -0.561 & 0.141 & $7.68 \mathrm{E}-03$ \\
\hline GTPase, IMAP family member 4 & 55303 & GIMAP4 & -0.558 & 0.188 & $2.48 \mathrm{E}-02$ \\
\hline dachshund homolog 1 (Drosophila) & 1602 & $\mathrm{DACH} 1$ & -0.555 & 0.116 & 2.95E-03 \\
\hline Rho family GTPase 1 & 27289 & RND1 & -0.552 & 0.175 & $1.90 \mathrm{E}-02$ \\
\hline histone cluster 1 , H2ai & 8329 & HIST1H2AI & -0.551 & 0.116 & $2.95 \mathrm{E}-03$ \\
\hline microtubule associated tumor suppressor 1 & 57509 & MTUS1 & -0.533 & 0.156 & $1.21 \mathrm{E}-02$ \\
\hline microRNA 100 & 406892 & MIR100 & -0.532 & 0.197 & 3.23E-02 \\
\hline NUAK family, SNF1-like kinase, 1 & 9891 & NUAK1 & -0.526 & 0.198 & 3.47E-02 \\
\hline BCL2-like 11 (apoptosis facilitator) & 10018 & BCL2L11 & -0.518 & 0.148 & $1.12 \mathrm{E}-02$ \\
\hline solute carrier family 40 (iron-regulated transporter), member 1 & 30061 & SLC40A1 & -0.517 & 0.155 & $1.40 \mathrm{E}-02$ \\
\hline TRAF-interacting protein with forkhead-associated domain & 92610 & TIFA & -0.515 & 0.187 & 3.14E-02 \\
\hline chromosome 11 open reading frame 82 & 220042 & C11orf82 & -0.512 & 0.219 & $5.66 \mathrm{E}-02$ \\
\hline growth differentiation factor 6 & 392255 & GDF6 & -0.482 & 0.213 & 6.34E-02 \\
\hline $\begin{array}{l}\text { family with sequence similarity } 117 \text {, member } B \\
\text { for }\end{array}$ & 150864 & FAM117B & -0.479 & 0.100 & $2.95 \mathrm{E}-03$ \\
\hline phosphodiesterase 7B & 27115 & PDE7B & -0.476 & 0.169 & 3.10E-02 \\
\hline Cdk5 and Abl enzyme substrate 1 & 91768 & CABLES1 & -0.474 & 0.144 & $1.52 \mathrm{E}-02$ \\
\hline microRNA 199a-2 & 406977 & MIR199A2 & -0.473 & 0.140 & $1.30 \mathrm{E}-02$ \\
\hline SH3-domain GRB2-like (endophilin) interacting protein 1 & 84251 & SGIP1 & -0.471 & 0.168 & 3.10E-02 \\
\hline microRNA 34a & 407040 & MIR34A & -0.469 & 0.170 & 3.14E-02 \\
\hline phosphoinositide-3-kinase interacting protein 1 & 113791 & PIK3IP1 & -0.469 & 0.227 & 8.87E-02 \\
\hline KAT8 regulatory NSL complex subunit 1 -like & 151050 & KANSL1L & -0.462 & 0.213 & 7.33E-02 \\
\hline programmed cell death 4 (neoplastic transformation inhibitor) & 27250 & PDCD4 & -0.462 & 0.089 & $1.96 \mathrm{E}-03$ \\
\hline butyrophilin, subfamily 3 , member A1 & 11119 & BTN3A1 & -0.460 & 0.122 & $9.92 \mathrm{E}-03$ \\
\hline dehydrogenase/reductase (SDR family) member 3 & 9249 & DHRS3 & -0.457 & 0.154 & $2.48 \mathrm{E}-02$ \\
\hline $\mathrm{H} 3$ histone, family $3 \mathrm{~A}$ & 3020 & H3F3A & -0.452 & 0.169 & 3.34E-02 \\
\hline aminoadipate-semialdehyde synthase & 10157 & AASS & -0.451 & 0.177 & 4.15E-02 \\
\hline R-spondin 3 & 84870 & RSPO3 & -0.447 & 0.137 & $1.53 \mathrm{E}-02$ \\
\hline zinc finger protein 704 & 619279 & ZNF704 & -0.440 & 0.195 & 6.43E-02 \\
\hline microRNA 216a & 406998 & MIR216A & -0.438 & 0.184 & $5.48 \mathrm{E}-02$ \\
\hline histone cluster $1, \mathrm{H} 2 \mathrm{bg}$ & 8339 & HIST1H2BG & -0.437 & 0.133 & $1.52 \mathrm{E}-02$ \\
\hline brain-derived neurotrophic factor & 627 & BDNF & -0.436 & 0.142 & 2.05E-02 \\
\hline interferon induced with helicase $\mathrm{C}$ domain 1 & 64135 & $\mathrm{IFIH} 1$ & -0.434 & 0.156 & 3.14E-02 \\
\hline myotubularin related protein 4 & 9110 & MTMR4 & -0.434 & 0.121 & $1.12 \mathrm{E}-02$ \\
\hline SATB homeobox 1 & 6304 & SATB1 & -0.432 & 0.177 & 4.90E-02 \\
\hline transmembrane protein 144 & 55314 & TMEM144 & -0.431 & 0.178 & $5.14 \mathrm{E}-02$ \\
\hline apolipoprotein L, 3 & 80833 & APOL3 & -0.429 & 0.145 & $2.54 \mathrm{E}-02$ \\
\hline $\begin{array}{l}\text { potassium intermediate/small conductance calcium-activated } \\
\text { channel, subfamily } \mathrm{N} \text {, member } 2\end{array}$ & 3781 & KCNN2 & -0.429 & 0.214 & $9.84 \mathrm{E}-02$ \\
\hline nuclear receptor subfamily 1 , group $D$, member 2 & 9975 & NR1D2 & -0.429 & 0.124 & $1.18 \mathrm{E}-02$ \\
\hline latexin & 56925 & LXN & -0.425 & 0.190 & $6.72 \mathrm{E}-02$ \\
\hline histone cluster $1, \mathrm{H} 3 \mathrm{e}$ & 8353 & HIST1H3E & -0.421 & 0.127 & $1.50 \mathrm{E}-02$ \\
\hline phosphodiesterase 3A, cGMP-inhibited & 5139 & PDE3A & -0.419 & 0.207 & $9.61 \mathrm{E}-02$ \\
\hline nuclear factor $\mathrm{I} / \mathrm{A}$ & 4774 & NFIA & -0.418 & 0.164 & 4.13E-02 \\
\hline chromosome 5 open reading frame 4 & 10826 & C5orf4 & -0.417 & 0.168 & 4.50E-02 \\
\hline Sp4 transcription factor & 6671 & SP4 & -0.417 & 0.141 & $2.48 \mathrm{E}-02$ \\
\hline nuclear receptor coactivator 7 & 135112 & NCOA7 & -0.414 & 0.145 & 3.00E-02 \\
\hline DnaJ (Hsp40) homolog, subfamily B, member 4 & 11080 & DNAJB4 & -0.413 & 0.123 & $1.31 \mathrm{E}-02$ \\
\hline $\begin{array}{l}\text { apolipoprotein B mRNA editing enzyme, catalytic polypeptide- } \\
\text { like } 3 \mathrm{~F}\end{array}$ & 200316 & APOBEC3F & -0.413 & 0.145 & 3.00E-02 \\
\hline $\begin{array}{l}\text { phospholipid scramblase } 4 \\
\text { butyrophilin, subfamily } 3 \text {, member A3 }\end{array}$ & $\begin{array}{l}57088 \\
10384\end{array}$ & $\begin{array}{l}\text { PLSCR4 } \\
\text { BTN3A3 }\end{array}$ & $\begin{array}{l}-0.412 \\
-0.410\end{array}$ & $\begin{array}{l}0.130 \\
0.150\end{array}$ & $\begin{array}{l}1.90 \mathrm{E}-02 \\
3.21 \mathrm{E}-02\end{array}$ \\
\hline
\end{tabular}


cell division cycle associated 7-like caspase recruitment domain family, member 16 pre-B-cell leukemia homeobox 1 KIAA1958

tropomodulin 1

nositol polyphosphate-4-phosphatase, type II 105kDa

cysteine-rich, angiogenic inducer, 61

F-box protein 32

butyrophilin, subfamily 3 , member $\mathrm{A} 2$

regulatory factor X, 2 (influences HLA class II expression)

GTPase, IMAP family member 8

yippee-like 1 (Drosophila)

actin, alpha 2, smooth muscle, aorta

protein phosphatase 1 , regulatory subunit $3 \mathrm{~B}$

guanylate binding protein 7

yippee-like 3 (Drosophila)

SLAIN motif family, member 1

GATS protein-like 1

transmembrane protein 140

protein phosphatase 1 , regulatory subunit $9 \mathrm{~A}$

dimethylarginine dimethylaminohydrolase 1

exocyst complex component 6

mastermind-like 2 (Drosophila)

KIAA0922

membrane associated guanylate kinase, WW and PDZ

domain containing 1

serum deprivation response

histone cluster $1, \mathrm{H} 2 \mathrm{bf}$

C-type lectin domain family 14 , member A

pyruvate dehydrogenase kinase, isozyme 4

neuronal regeneration related protein homolog (rat)

Cbl proto-oncogene, E3 ubiquitin protein ligase B

myeloid/lymphoid or mixed-lineage leukemia (trithorax homolog, Drosophila); translocated to, 3

zinc finger protein 48

guanosine monophosphate reductase

LGAP1 antisense RNA 1

FK506 binding protein 5

zinc finger, $\mathrm{CCHC}$ domain containing 18

transcription factor Dp-2 (E2F dimerization partner 2)

homeobox A6

LYR motif containing 1

SRY (sex determining region Y)-box 4

GA binding protein transcription factor, beta subunit 1

TRIM6-TRIM34 readthrough

guanylate binding protein 1, interferon-inducible

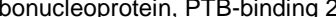

SMAD family member 1

lysophosphatidic acid receptor 6

kinesin family member $18 \mathrm{~A}$

CCR4-NOT transcription complex, subunit 6-like

MDS1 and EVI1 complex locus

sterile alpha motif domain containing 13

aldehyde dehydrogenase 6 family, member A1

solute carrier family 25 , member 30

phospholipase A2, group IVC (cytosolic, calciumindependent)

kelch-like 13 (Drosophila)

TBC1 domain family, member 22B

family with sequence similarity 84 , member $B$

vestigial like 3 (Drosophila)

centrosomal protein $19 \mathrm{kDa}$

protein kinase, cAMP-dependent, catalytic, beta

solute carrier family 2 (facilitated glucose transporter), member 12

mitochondria-localized glutamic acid-rich protein

golgi phosphoprotein 3-like

DIS3 mitotic control homolog (S. cerevisiae)-like

zinc finger protein 610

carnitine O-octanoyltransferase

Rho GTPase activating protein 29

Rho guanine nucleotide exchange factor (GEF) 37

regulatory factor $X, 5$ (influences HLA class II expression)

family with sequence similarity 43 , member $A$

amily with sequence similarity 149 , member B1

phosphodiesterase 4D, cAMP-specific

G protein-coupled receptor 125

sideroflexin 2

phospholipid scramblase 1

chromosome 10 open reading frame 114

\begin{tabular}{|c|c|c|c|c|}
\hline 55536 & CDCA7L & -0.408 & 0.172 & 5.51E-02 \\
\hline 114769 & CARD16 & -0.407 & 0.171 & 5.48E-02 \\
\hline 5087 & PBX1 & -0.407 & 0.185 & 7.04E-02 \\
\hline 158405 & KIAA1958 & -0.406 & 0.190 & 7.74E-02 \\
\hline 7111 & TMOD1 & -0.406 & 0.184 & 6.89E-02 \\
\hline 8821 & INPP4B & -0.406 & 0.184 & 6.97E-02 \\
\hline 3491 & CYR61 & -0.399 & 0.142 & $3.10 \mathrm{E}-02$ \\
\hline 114907 & FBX032 & -0.399 & 0.142 & $3.10 \mathrm{E}-02$ \\
\hline 11118 & BTN3A2 & -0.399 & 0.101 & $7.72 \mathrm{E}-03$ \\
\hline 5990 & RFX2 & -0.398 & 0.161 & 4.66E-02 \\
\hline 155038 & GIMAP8 & -0.398 & 0.176 & 6.43E-02 \\
\hline 29799 & YPEL1 & -0.394 & 0.154 & 4.07E-02 \\
\hline 59 & ACTA2 & -0.393 & 0.111 & $1.12 \mathrm{E}-02$ \\
\hline 79660 & PPP1R3B & -0.391 & 0.133 & $2.58 \mathrm{E}-02$ \\
\hline 388646 & GBP7 & -0.390 & 0.126 & $2.01 \mathrm{E}-02$ \\
\hline 83719 & YPEL3 & -0.387 & 0.130 & $2.48 \mathrm{E}-02$ \\
\hline 122060 & SLAIN1 & -0.385 & 0.186 & 8.78E-02 \\
\hline 389523 & GATSL1 & -0.385 & 0.123 & 1.97E-02 \\
\hline 55281 & TMEM140 & -0.385 & 0.117 & $1.52 \mathrm{E}-02$ \\
\hline 55607 & PPP1R9A & -0.384 & 0.113 & 1.23E-02 \\
\hline 23576 & $\mathrm{DDAH} 1$ & -0.384 & 0.137 & 3.10E-02 \\
\hline 54536 & EXOC6 & -0.383 & 0.163 & 5.57E-02 \\
\hline 84441 & MAML2 & -0.383 & 0.133 & $2.84 \mathrm{E}-02$ \\
\hline 23240 & KIAA0922 & -0.380 & 0.143 & $3.45 E-02$ \\
\hline 9223 & MAGI1 & -0.380 & 0.145 & 3.73E-02 \\
\hline 8436 & SDPR & -0.379 & 0.128 & $2.48 \mathrm{E}-02$ \\
\hline 8343 & HIST1H2BF & -0.372 & 0.158 & 5.57E-02 \\
\hline 161198 & CLEC14A & -0.371 & 0.120 & $2.02 \mathrm{E}-02$ \\
\hline 5166 & PDK4 & -0.370 & 0.164 & $6.43 \mathrm{E}-02$ \\
\hline 9315 & NREP & -0.367 & 0.167 & $7.06 \mathrm{E}-02$ \\
\hline 868 & CBLB & -0.365 & 0.117 & 2.01E-02 \\
\hline 4300 & MLLT3 & -0.363 & 0.141 & 3.98E-02 \\
\hline 197407 & ZNF48 & -0.361 & 0.104 & 1.14E-02 \\
\hline 2766 & GMPR & -0.359 & 0.098 & $1.02 \mathrm{E}-02$ \\
\hline 649446 & DLGAP1-AS1 & -0.358 & 0.149 & 5.39E-02 \\
\hline 2289 & FKBP5 & -0.352 & 0.136 & 3.97E-02 \\
\hline 644353 & $\mathrm{ZCCHC} 18$ & -0.351 & 0.152 & 5.94E-02 \\
\hline 7029 & TFDP2 & -0.351 & 0.085 & 6.37E-03 \\
\hline 3203 & HOXA6 & -0.344 & 0.151 & $6.10 \mathrm{E}-02$ \\
\hline 57149 & LYRM1 & -0.342 & 0.135 & 4.15E-02 \\
\hline 6659 & SOX4 & -0.341 & 0.152 & 6.66E-02 \\
\hline 2553 & GABPB1 & -0.341 & 0.124 & $3.14 \mathrm{E}-02$ \\
\hline 445372 & TRIM6-TRIM34 & -0.338 & 0.169 & $9.89 \mathrm{E}-02$ \\
\hline 2633 & GBP1 & -0.336 & 0.132 & 4.15E-02 \\
\hline 55225 & RAVER2 & -0.335 & 0.162 & 8.96E-02 \\
\hline 4086 & SMAD1 & -0.333 & 0.142 & 5.66E-02 \\
\hline 10161 & LPAR6 & -0.329 & 0.157 & $8.49 \mathrm{E}-02$ \\
\hline 81930 & KIF18A & -0.328 & 0.164 & $9.77 \mathrm{E}-02$ \\
\hline 246175 & CNOT6L & -0.327 & 0.151 & 7.36E-02 \\
\hline 2122 & MECOM & -0.326 & 0.134 & 4.91E-02 \\
\hline 148418 & SAMD13 & -0.325 & 0.137 & 5.51E-02 \\
\hline 4329 & ALDH6A1 & -0.322 & 0.116 & $3.14 \mathrm{E}-02$ \\
\hline 253512 & SLC25A30 & -0.321 & 0.117 & $3.14 \mathrm{E}-02$ \\
\hline 8605 & PLA2G4C & -0.320 & 0.100 & $1.81 \mathrm{E}-02$ \\
\hline 90293 & KLHL13 & -0.319 & 0.132 & $5.21 \mathrm{E}-02$ \\
\hline 55633 & TBC1D22B & -0.313 & 0.141 & $6.81 \mathrm{E}-02$ \\
\hline 157638 & FAM84B & -0.312 & 0.144 & 7.33E-02 \\
\hline 389136 & VGLL3 & -0.312 & 0.155 & $9.77 \mathrm{E}-02$ \\
\hline 84984 & CEP19 & -0.311 & 0.156 & $9.94 \mathrm{E}-02$ \\
\hline 5567 & PRKACB & -0.308 & 0.121 & 4.15E-02 \\
\hline 154091 & SLC2A12 & -0.307 & 0.148 & 8.71E-02 \\
\hline 84709 & MGARP & -0.305 & 0.111 & $3.14 \mathrm{E}-02$ \\
\hline 55204 & GOLPH3L & -0.305 & 0.135 & 6.43E-02 \\
\hline 115752 & DIS3L & -0.305 & 0.111 & 3.17E-02 \\
\hline 162963 & ZNF610 & -0.303 & 0.097 & 1.97E-02 \\
\hline 54677 & CROT & -0.303 & 0.142 & 7.86E-02 \\
\hline 9411 & ARHGAP29 & -0.301 & 0.121 & $4.50 \mathrm{E}-02$ \\
\hline 389337 & ARHGEF37 & -0.301 & 0.124 & $5.20 \mathrm{E}-02$ \\
\hline 5993 & RFX5 & -0.300 & 0.109 & $3.14 \mathrm{E}-02$ \\
\hline 131583 & FAM43A & -0.300 & 0.127 & 5.57E-02 \\
\hline 317662 & FAM149B1 & -0.296 & 0.133 & 6.80E-02 \\
\hline 5144 & PDE4D & -0.296 & 0.117 & $4.20 \mathrm{E}-02$ \\
\hline 166647 & GPR125 & -0.294 & 0.122 & 5.19E-02 \\
\hline 118980 & SFXN2 & -0.292 & 0.135 & 7.46E-02 \\
\hline 5359 & PLSCR1 & -0.291 & 0.095 & $2.22 \mathrm{E}-02$ \\
\hline 399726 & C10orf114 & -0.290 & 0.106 & 3.17E-02 \\
\hline
\end{tabular}


proline-rich nuclear receptor coactivator 1 ral guanine nucleotide dissociation stimulator-like 1

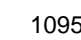

zinc finger and SCAN domain containing 16

forkhead box N3

signal transducer and activator of transcription $1,91 \mathrm{kDa}$

Rembrane protein complex subunit 2

KIAA1107

long intergenic non-protein coding RNA 478

homeobox A4

G protein-coupled receptor 146

SH3 domain containing 19

adducin 3 (gamma)

Rho-related BTB domain containing 3

ST6 beta-galactosamide alpha-2,6-sialyltranferase 1

uracil-DNA glycosylase

dynamin binding protein

hydroxysteroid (17-beta) dehydrogenase 7

WD repeat domain, phosphoinositide interacting 1

histone cluster $1, \mathrm{H} 3 \mathrm{~h}$

methylcrotonoyl-CoA carboxylase 1 (alpha)

NADH dehydrogenase (ubiquinone) 1 alpha subcomplex, 2, $8 \mathrm{kDa}$

leucine-rich repeats and immunoglobulin-like domains 1

WW domain binding protein 1-like

ubiquitin specific peptidase 28

syntaxin binding protein 4

translocase of inner mitochondrial membrane 8 homolog $B$ (yeast)

erythrocyte membrane protein band 4.1 (elliptocytosis $1, \mathrm{RH}$ linked)

chromosome 6 open reading frame 141

caspase 2, apoptosis-related cysteine peptidase

histone cluster $1, \mathrm{H} 4 \mathrm{~d}$

guanylate cyclase 1 , soluble, alpha 3

histone cluster 1 , H3d

zinc finger and SCAN domain containing 2

Bcl2 modifying factor

cyclin M3

kinesin family member $13 \mathrm{~A}$

Rho guanine nucleotide exchange factor (GEF) 28

OMA1 zinc metallopeptidase homolog (S. cerevisiae)

t-complex 11 (mouse)-like 2

tumor protein p53 inducible nuclear protein 1

KIAA1328

family with sequence similarity 63 , member $A$

TBC1 domain containing kinase

CREB3 regulatory factor

RAB30, member RAS oncogene family

integrator complex subunit 8

family with sequence similarity 198 , member $B$

chromosome 17 open reading frame 28

microRNA 93

transmembrane protein 117

minichromosome maintenance complex component 7

leucine rich repeat containing 8 family, member $B$

apolipoprotein B mRNA editing enzyme, catalytic polypeptidelike 3F

DnaJ (Hsp40) homolog, subfamily C, member 6

TRAF family member-associated NFKB activator

phosphodiesterase 4B, cAMP-specific

ring finger protein 44

phosphofructokinase, muscle

opsin 3

solute carrier family 35 , member $\mathrm{E} 2 \mathrm{~B}$

HMG-box transcription factor 1

dystrophin

tripartite motif containing 5

CTD (carboxy-terminal domain, RNA polymerase II, polypeptide A) small phosphatase 2

histone cluster $1, \mathrm{H} 2 \mathrm{ag}$

filamin A interacting protein 1

XRCC6 binding protein 1

solute carrier family 1 (neuronal/epithelial high affinity glutamate transporter, system Xag), member

ankyrin repeat domain 50

zinc finger protein 254

family with sequence similarity 111 , member $A$

LIM and senescent cell antigen-like domains 3

kelch-like 23 (Drosophila)

uridine phosphorylase 1
80345

1112
6772

9694

23285

388815

3201

115330

152503

120

22836

6480

7374

23268

51478

55062

8357

56922

4695

26018

54838

57646

252983

26521

2035

135398

835

8360

2982

8351

54993

9042

26505

63971

64283

115209

255394

94241

57536

55793

93627

153222

27314

55656

51313

283987

407050

84216

4176

23507

200316

9829

10010

5142

22838

5213

23596

728661

26959

1756

85363

10106

8969

27145

91419

6505

57182

9534

63901

96626

151230

7378
PNRC1

RGL1

ZSCAN16

FOXN3

STAT

MC2

KIAA1107

LINC00478

HOXA4

GPR146

SH3D19

ADD3

RHOBTB3

ST6GAL1

UNG

DNMBP

HSD17B7

WIPI1

HIST1H3H

MCCC1

NDUFA2

LRIG1

WBP1L

USP28

STXBP4

TIMM8B

EPB41

C6orf141

CASP2

HIST1H4D

GUCY1A3

HIST1H3D

ZSCAN2

BMF

CNNM3

KIF13A

ARHGEF28

OMA1

TCP11L2

TP53INP1

KIAA1328

FAM63A

TBCK

CREBRF

RAB30

INTS8

FAM198B

C17orf28

MIR93

TMEM117

MCM7

LRRC8B

APOBEC3F

DNAJC6

TANK

PDE4B

RNF44

PFKM

OPN3

SLC35E2B

HBP1

DMD

TRIM5

CTDSP2

HIST1H2AG

FILIP1

XRCC6BP1

SLC1A1

ANKRD50

ZNF254

FAM111A

LIMS3

KLHL23

UPP1

$-0.290$

$-0.288$

$-0.288$

$-0.288$

$-0.287$

$-0.287$

$-0.287$

$-0.287$

$-0.285$

$-0.285$

$-0.285$

$-0.285$

$-0.285$

$-0.285$

$-0.284$

$-0.284$

$-0.284$

$-0.283$

$-0.283$

$-0.282$

$-0.280$

$-0.280$

$-0.279$

$-0.279$

$-0.278$

$-0.276$

$-0.276$

$-0.275$

$-0.274$

$-0.274$

$-0.271$

$-0.270$

$-0.269$

$-0.269$

$-0.266$

$-0.265$

$-0.263$

$-0.262$

$-0.261$

$-0.260$

$-0.258$

$-0.256$

$-0.256$

$-0.255$

$-0.254$

$-0.254$

$-0.253$

$-0.250$

$-0.250$

$-0.249$

$-0.249$

$-0.249$

$-0.248$

$-0.243$

$-0.241$

$-0.241$

$-0.240$

$-0.240$

$-0.240$

$-0.240$

$-0.239$

$-0.237$

$-0.237$ 
NMDA receptor regulated 2

polymerase (DNA directed), beta

synovial sarcoma, $\mathrm{X}$ breakpoint 2

transcription factor 4

PDZ and LIM domain 5

sestrin 1

SEC14 and spectrin domains 1

stearoyl-CoA desaturase 5

myeloid/lymphoid or mixed-lineage leukemia (trithorax

homolog, Drosophila); translocated to, 1

methionine adenosyltransferase II, beta

synapse defective 1, Rho GTPase, homolog 2 (C. elegans)

zinc finger protein 260

chromosome 2 open reading frame $27 \mathrm{~A}$

eukaryotic translation initiation factor 2 alpha kinase 4

mitogen-activated protein kinase kinase kinase 3

synuclein, alpha (non A4 component of amyloid precursor)

centrosomal protein $41 \mathrm{kDa}$

mex-3 homolog B (C. elegans)

ubiquitin specific peptidase 49

SEC14-like 1 (S. cerevisiae)

nuclear mitotic apparatus protein 1

uncharacterized LOC441204

ATP/GTP binding protein-like 5

family with sequence similarity 115 , member $C$

hematopoietic prostaglandin D synthase

Hermansky-Pudlak syndrome 3

cysteine conjugate-beta lyase, cytoplasmic

adaptor-related protein complex 3 , mu 2 subunit

GRAM domain containing $1 \mathrm{~A}$

damage-specific DNA binding protein 2, 48kDa

\begin{tabular}{lllll}
79664 & NARG2 & -0.229 & 0.103 & $6.89 \mathrm{E}-02$ \\
5423 & POLB & -0.228 & 0.097 & $5.57 \mathrm{E}-02$ \\
6757 & SSX2 & -0.227 & 0.097 & $5.59 \mathrm{E}-02$ \\
6925 & TCF4 & -0.224 & 0.105 & $7.92 \mathrm{E}-02$ \\
10611 & PDLIM5 & -0.224 & 0.104 & $7.47 \mathrm{E}-02$ \\
27244 & SESN1 & -0.223 & 0.093 & $5.45 \mathrm{E}-02$ \\
91404 & SESTD1 & -0.222 & 0.090 & $4.63 \mathrm{E}-02$ \\
79966 & SCD5 & -0.221 & 0.102 & $7.26 \mathrm{E}-02$ \\
4298 & MLLT1 & -0.221 & 0.110 & $9.72 \mathrm{E}-02$ \\
27430 & MAT2B & -0.221 & 0.106 & $8.50 \mathrm{E}-02$ \\
84144 & SYDE2 & -0.220 & 0.104 & $7.97 \mathrm{E}-02$ \\
339324 & ZNF260 & -0.220 & 0.110 & $9.84 \mathrm{E}-02$ \\
29798 & C2orf27A & -0.219 & 0.108 & $9.71 \mathrm{E}-02$ \\
440275 & EIF2AK4 & -0.216 & 0.094 & $6.01 \mathrm{E}-02$ \\
4215 & MAP3K3 & -0.215 & 0.106 & $9.44 \mathrm{E}-02$ \\
6622 & SNCA & -0.211 & 0.097 & $7.17 \mathrm{E}-02$ \\
95681 & CEP41 & -0.209 & 0.096 & $7.06 \mathrm{E}-02$ \\
84206 & MEX3B & -0.209 & 0.094 & $6.80 \mathrm{E}-02$ \\
25862 & USP49 & -0.206 & 0.092 & $6.75 \mathrm{E}-02$ \\
6397 & SEC14L1 & -0.202 & 0.100 & $9.69 \mathrm{E}-02$ \\
4926 & NUMA1 & -0.201 & 0.098 & $9.01 \mathrm{E}-02$ \\
441204 & LOC441204 & -0.199 & 0.099 & $9.77 \mathrm{E}-02$ \\
60509 & AGBL5 & -0.198 & 0.090 & $7.06 \mathrm{E}-02$ \\
285966 & FAM115C & -0.197 & 0.089 & $6.78 \mathrm{E}-02$ \\
27306 & HPGDS & -0.194 & 0.097 & $9.77 \mathrm{E}-02$ \\
84343 & HPS3 & -0.193 & 0.093 & $8.85 \mathrm{E}-02$ \\
883 & CCBL1 & -0.190 & 0.088 & $7.47 \mathrm{E}-02$ \\
10947 & AP3M2 & -0.190 & 0.092 & $8.96 \mathrm{E}-02$ \\
57655 & GRAMD1A & -0.185 & 0.090 & $9.20 \mathrm{E}-02$ \\
1643 & DDB2 & -0.184 & 0.090 & $9.08 \mathrm{E}-02$ \\
\hline & & & &
\end{tabular}




\section{Online table 5.II}

Online Table 5.II. All differentially regulated genes between non-reversed (increased shear stress) versus control (steady shear stress) conditions with FDR<0.10

\begin{tabular}{|c|c|c|c|c|c|}
\hline Gene Name & Entrez ID & Symbol & $\log _{2} F C$ & $\begin{array}{c} \pm 95 \% \\
\text { C.I. }\end{array}$ & $F D R$ \\
\hline \multicolumn{6}{|l|}{ A. Upregulated Genes } \\
\hline 1-acylglycerol-3-phosphate O-acyltransferase 9 & 84803 & AGPAT9 & 0.652 & 0.184 & 6.61E-02 \\
\hline Thrombomodulin & 7056 & THBD & 0.606 & 0.127 & 1.63E-02 \\
\hline carbonic anhydrase XIII & 377677 & CA13 & 0.588 & 0.213 & 9.97E-02 \\
\hline KIT ligand & 4254 & KITLG & 0.581 & 0.205 & 9.97E-02 \\
\hline kelch repeat and BTB (POZ) domain containing 8 & 84541 & KBTBD8 & 0.571 & 0.114 & $1.63 \mathrm{E}-02$ \\
\hline cell division cycle 6 homolog (S. cerevisiae) & 990 & CDC6 & 0.549 & 0.143 & $6.13 \mathrm{E}-02$ \\
\hline nephroblastoma overexpressed & 4856 & NOV & 0.478 & 0.129 & $6.13 \mathrm{E}-02$ \\
\hline $\begin{array}{l}\text { sema domain, immunoglobulin domain (lg), transmembrane } \\
\text { domain (TM) and short cytoplasmic domain, (semaphorin) } \\
\text { 4B }\end{array}$ & 10509 & SEMA4B & 0.429 & 0.125 & $6.61 \mathrm{E}-02$ \\
\hline odz, odd Oz/ten-m homolog 3 (Drosophila) & 55714 & ODZ3 & 0.394 & 0.143 & 9.97E-02 \\
\hline DnaJ (Hsp40) homolog, subfamily B, member 9 & 4189 & DNAJB9 & 0.388 & 0.131 & 9.97E-02 \\
\hline Rap guanine nucleotide exchange factor (GEF) 5 & 9771 & RAPGEF5 & 0.385 & 0.139 & 9.97E-02 \\
\hline MAX dimerization protein 1 & 4084 & MXD1 & 0.380 & 0.108 & $6.61 \mathrm{E}-02$ \\
\hline $\begin{array}{l}\text { pleckstrin homology domain containing, family G (with RhoGef } \\
\text { domain) member } 1\end{array}$ & 57480 & PLEKHG1 & 0.368 & 0.117 & $7.90 \mathrm{E}-02$ \\
\hline pentraxin 3 , long & 5806 & РTX3 & 0.362 & 0.129 & 9.97E-02 \\
\hline forkhead box $\mathrm{F} 1$ & 2294 & FOXF1 & 0.346 & 0.124 & 9.97E-02 \\
\hline Smoothelin & 6525 & SMTN & 0.333 & 0.099 & $6.61 \mathrm{E}-02$ \\
\hline UDP-glucose 6-dehydrogenase & 7358 & UGDH & 0.327 & 0.118 & 9.97E-02 \\
\hline small cell adhesion glycoprotein & 57228 & SMAGP & 0.317 & 0.113 & 9.97E-02 \\
\hline KIAA1024 & 23251 & KIAA1024 & 0.307 & 0.097 & 7.90E-02 \\
\hline sphingomyelin synthase 2 & 166929 & SGMS2 & 0.291 & 0.100 & 9.97E-02 \\
\hline dual specificity phosphatase 1 & 1843 & DUSP1 & 0.290 & 0.103 & 9.97E-02 \\
\hline \multicolumn{6}{|l|}{ B. Downregulated Genes } \\
\hline $\begin{array}{l}\text { 5-hydroxytryptamine (serotonin) receptor 2B, G protein- } \\
\text { coupled }\end{array}$ & 3357 & HTR2B & -0.823 & 0.292 & $9.97 \mathrm{E}-02$ \\
\hline microRNA 519a-2 & 574500 & MIR519A2 & -0.717 & 0.215 & 6.61E-02 \\
\hline guanylate binding protein 4 & 115361 & GBP4 & -0.712 & 0.239 & 9.97E-02 \\
\hline guanylate binding protein 2 , interferon-inducible & 2634 & GBP2 & -0.651 & 0.195 & $6.61 \mathrm{E}-02$ \\
\hline ADP-ribosyltransferase 4 (Dombrock blood group) & 420 & ART4 & -0.570 & 0.185 & 8.69E-02 \\
\hline dehydrogenase/reductase (SDR family) member 3 & 9249 & DHRS3 & -0.508 & 0.154 & $6.70 \mathrm{E}-02$ \\
\hline sestrin 3 & 143686 & SESN3 & -0.492 & 0.155 & 7.90E-02 \\
\hline Rho family GTPase 1 & 27289 & RND1 & -0.483 & 0.175 & 9.97E-02 \\
\hline microRNA 199a-2 & 406977 & MIR199A2 & -0.477 & 0.140 & 6.61E-02 \\
\hline butyrophilin, subfamily 3 , member $A 1$ & 11119 & BTN3A1 & -0.453 & 0.122 & $6.13 E-02$ \\
\hline DLGAP1 antisense RNA 1 & 649446 & DLGAP1-AS1 & -0.419 & 0.149 & 9.97E-02 \\
\hline apolipoprotein L, 3 & 80833 & APOL3 & -0.411 & 0.145 & 9.97E-02 \\
\hline G protein-coupled receptor 146 & 115330 & GPR146 & -0.375 & 0.121 & 8.69E-02 \\
\hline microsomal glutathione S-transferase 2 & 4258 & MGST2 & -0.373 & 0.134 & 9.97E-02 \\
\hline transmembrane protein 140 & 55281 & TMEM140 & -0.369 & 0.117 & 7.90E-02 \\
\hline guanylate binding protein 7 & 388646 & GBP7 & -0.367 & 0.126 & 9.97E-02 \\
\hline histone cluster 1, H2ai & 8329 & HIST1H2AI & -0.343 & 0.116 & 9.97E-02 \\
\hline protein phosphatase 1 , regulatory subunit $9 \mathrm{~A}$ & 55607 & PPP1R9A & -0.335 & 0.113 & 9.97E-02 \\
\hline KIAA1107 & 23285 & KIAA1107 & -0.334 & 0.113 & 9.97E-02 \\
\hline phosphodiesterase 4D, cAMP-specific & 5144 & PDE4D & -0.333 & 0.117 & 9.97E-02 \\
\hline zinc finger protein 610 & 162963 & ZNF610 & -0.326 & 0.097 & $6.61 \mathrm{E}-02$ \\
\hline transcription factor Dp-2 (E2F dimerization partner 2) & 7029 & TFDP2 & -0.319 & 0.085 & $6.13 \mathrm{E}-02$ \\
\hline phospholipase A2, group IVC (cytosolic, calcium-independent) & 8605 & PLA2G4C & -0.278 & 0.100 & 9.97E-02 \\
\hline guanosine monophosphate reductase & 2766 & GMPR & -0.272 & 0.098 & 9.97E-02 \\
\hline sestrin 1 & 27244 & SESN1 & -0.265 & 0.093 & 9.97E-02 \\
\hline
\end{tabular}




\section{Online Table 5.III}

Online Table 5.III. Gene ontology analysis for over-representation of molecular function.

\begin{tabular}{|c|c|c|c|c|}
\hline Description & P-value & $\begin{array}{c}\text { FDR } \\
\text { q-value }\end{array}$ & $\begin{array}{l}\text { Enrichment } \\
\text { Score }\end{array}$ & Enriched Genes in Pathway \\
\hline \multicolumn{5}{|l|}{ A. Non-reversal v Control } \\
\hline $\begin{array}{l}\text { oxidoreductase activity, acting } \\
\text { on the CH-NH group of donors, } \\
\text { NAD or NADP as acceptor }\end{array}$ & $6.66 \mathrm{E}-04$ & $1.00 \mathrm{E}+00$ & 9.57 & AASS, MTHFR, PYCR1, MTHFD2 \\
\hline \multicolumn{5}{|l|}{ B. Reversal v Control } \\
\hline $\begin{array}{l}\text { 3',5'-cyclic-AMP } \\
\text { phosphodiesterase activity }\end{array}$ & 8.29E-05 & $3.22 \mathrm{E}-01$ & 15.42 & PDE4D, PDE4B, PDE3A, PDE7B \\
\hline $\begin{array}{l}\text { nucleoside-triphosphatase } \\
\text { regulator activity }\end{array}$ & $1.07 \mathrm{E}-04$ & 2.07E-01 & 2.25 & $\begin{array}{l}\text { ARHGAP18, RAPGEF5, CYTH1, TBC1D2, } \\
\text { GRPEL1, PREX2, ARHGAP29, } \\
\text { TBC1D22B, KIAA1244, HTR2B, SYDE2, } \\
\text { STARD8, SIPA1L3, PLEKHG5, RGL1, } \\
\text { TBCK, RASA4, PLEKHG1, PSD3, } \\
\text { ARHGAP28, DNMBP, RASGEF1B, } \\
\text { ARHGEF37, SH2D3C, TBXA2R, DOCK11 }\end{array}$ \\
\hline GTPase regulator activity & $1.73 \mathrm{E}-04$ & 2.25E-01 & 2.23 & $\begin{array}{l}\text { ARHGAP18, RAPGEF5, CYTH1, TBC1D2, } \\
\text { PREX2, ARHGAP29, TBC1D22B, } \\
\text { KIAA1244, HTR2B, SYDE2, STARD8, } \\
\text { SIPA1L3, PLEKHG5, RGL1, TBCK, } \\
\text { RASA4, PLEKHG1, PSD3, ARHGAP28, } \\
\text { DNMBP, RASGEF1B, ARHGEF37, } \\
\text { SH2D3C, TBXA2R, DOCK11 }\end{array}$ \\
\hline small GTPase regulator activity & 2.07E-04 & 2.01E-01 & 2.53 & $\begin{array}{l}\text { RASA4, RAPGEF5, PLEKHG1, CYTH1, } \\
\text { TBC1D2, PSD3, PREX2, ARHGAP29, } \\
\text { TBC1D22B, KIAA1244, RASGEF1B, } \\
\text { DNMBP, HTR2B, ARHGEF37, SYDE2, } \\
\text { TBCK, PLEKHG5, RGL1, DOCK11 }\end{array}$ \\
\hline $\begin{array}{l}\text { guanyl-nucleotide exchange } \\
\text { factor activity }\end{array}$ & 2.45E-04 & $1.91 \mathrm{E}-01$ & 3.01 & $\begin{array}{l}\text { RAPGEF5, CYTH1, PLEKHG1, PSD3, } \\
\text { PREX2, KIAA1244, DNMBP, RASGEF1B, } \\
\text { ARHGEF37, SH2D3C, TBXA2R, RGL1, } \\
\text { PLEKHG5, DOCK11 }\end{array}$ \\
\hline $\begin{array}{l}\text { 3',5'-cyclic-nucleotide } \\
\text { phosphodiesterase activity }\end{array}$ & $3.25 \mathrm{E}-04$ & 2.10E-01 & 8.03 & PDE4D, PDE4B, PDE3A, PDE2A, PDE7B \\
\hline $\begin{array}{l}\text { RNA polymerase II core } \\
\text { promoter proximal region } \\
\text { sequence-specific DNA binding } \\
\text { transcription factor activity }\end{array}$ & 3.53E-04 & $1.96 \mathrm{E}-01$ & 4.08 & $\begin{array}{l}\text { RCOR1, NR4A1, CREB5, PLSCR1, KLF4, } \\
\text { DACH1, TCF4, SOX4, ARNTL }\end{array}$ \\
\hline $\begin{array}{l}\text { cyclic-nucleotide } \\
\text { phosphodiesterase activity }\end{array}$ & 3.97E-04 & $1.93 \mathrm{E}-01$ & 7.71 & PDE4D, PDE4B, PDE3A, PDE2A, PDE7B \\
\hline $\begin{array}{l}\text { sequence-specific DNA binding } \\
\text { RNA polymerase II } \\
\text { transcription factor activity }\end{array}$ & $5.28 \mathrm{E}-04$ & $2.28 \mathrm{E}-01$ & 2.58 & $\begin{array}{l}\text { RCOR1, NR4A1, RARB, DACH1, TCF4, } \\
\text { NR1D2, KLF11, ARNTL, STAT1, CREB5, } \\
\text { PLSCR1, FOXN2, KLF4, SOX4, MEF2A, } \\
\text { FOXN3 }\end{array}$ \\
\hline reelin receptor activity & $6.72 \mathrm{E}-04$ & 2.61E-01 & 38.55 & VLDLR, LRP8 \\
\hline
\end{tabular}

Represents gene ontology clustering from top 500 genes from each group (reversal versus control and non-reversal versus control) as ranked by $p$-value 


\section{Online Table 5.IV}

Online Table 5.IV. Ingenuity Pathways Analysis significant cannonical pathways

\begin{tabular}{|c|c|c|c|}
\hline Pathway & P-value & Ratio & Molecules \\
\hline \multicolumn{4}{|l|}{ A. Reversal v Control } \\
\hline Cellular Effects of Sildenafil (Viagra) & 1.17E-03 & $7.8 \mathrm{E}-02$ & $\begin{array}{l}\text { PRKACB, PDE2A, KCNN2, GUCY1A3, } \\
\text { GPR37, PDE3A, ACTA2, ITPR3, PDE4B, } \\
\text { NOS3, PDE4D }\end{array}$ \\
\hline $\begin{array}{l}\text { Corticotropin } \\
\text { Signaling }\end{array}$ & $1.45 \mathrm{E}-03$ & 7.46E-02 & $\begin{array}{l}\text { PRKACB, SHH, GUCY1A3, BDNF, NPR1, } \\
\text { ITPR3, MEF2A, NR4A1, CREB5, NOS3 }\end{array}$ \\
\hline tRNA Splicing & 2.45E-03 & 1.19E-01 & PDE2A, PDE7B, PDE3A, PDE4B, PDE4D \\
\hline Protein Kinase A Signaling & 5.37E-03 & $5.29 \mathrm{E}-02$ & $\begin{array}{l}\text { PRKACB, AKAP12, SHH, TCF4, PDE2A, } \\
\text { H3F3A/H3F3B, YWHAH, PDE3A, PDE4B, } \\
\text { CREB5, NOS3, PDE4D, DHH, DUSP5, ADD3, } \\
\text { PDE7B, PTPRJ, DUSP1, ITPR3, EBI3 }\end{array}$ \\
\hline cAMP-mediated signaling & $6.17 \mathrm{E}-03$ & $6.36 \mathrm{E}-02$ & $\begin{array}{l}\text { AKAP12, PRKACB, SRC, GPR17, PDE2A, } \\
\text { PDE7B, DUSP1, PDE3A, TBXA2R, HTR1D, } \\
\text { PDE4B, PDE4D, CREB5, PKIG }\end{array}$ \\
\hline p38 MAPK Signaling & $6.46 \mathrm{E}-03$ & 7.89E-02 & $\begin{array}{l}\text { H3F3A/H3F3B, TIFA, DUSP1, PLA2G4C, } \\
\text { MEF2A, IL1R1, CREB5, STAT1, FAS }\end{array}$ \\
\hline Gap Junction Signaling & $1.55 \mathrm{E}-02$ & $5.85 \mathrm{E}-02$ & $\begin{array}{l}\text { PRKACB, SRC, HTR2B, NOV, GUCY1A3, } \\
\text { NPR1, ACTA2, TUBB2A, ITPR3, TUBA4A }\end{array}$ \\
\hline Sertoli Cell-Sertoli Cell Junction Signaling & $1.58 \mathrm{E}-02$ & 5.7E-02 & $\begin{array}{l}\text { PRKACB, SRC, EPB41, CLDN12, GUCY1A3, } \\
\text { CLDN1, ACTA2, TUBB2A, TUBA4A, NOS3, } \\
\text { MAP3K3 }\end{array}$ \\
\hline Relaxin Signaling & 1.91E-02 & 5.77E-02 & $\begin{array}{l}\text { PRKACB, PDE2A, PDE7B, GUCY1A3, PDE3A, } \\
\text { NPR1, PDE4B, NOS3, PDE4D }\end{array}$ \\
\hline$\beta$-alanine Degradation I & 3.09E-02 & $1.11 \mathrm{E}-01$ & ALDH6A1 \\
\hline $\begin{array}{l}\text { All-trans-decaprenyl } \quad \text { Diphosphate } \\
\text { Biosynthesis }\end{array}$ & 3.09E-02 & 2E-01 & PDSS1 \\
\hline Epithelial Adherens Junction Signaling & 3.31E-02 & 6.16E-02 & $\begin{array}{l}\text { SRC, DLL1, TCF4, MAGl1, LMO7, FGFR1, } \\
\text { ACTA2, TUBB2A, TUBA4A }\end{array}$ \\
\hline ERK5 Signaling & 3.55E-02 & 7.94E-02 & SRC, YWHAH, MEF2A, CREB5, MAP3K3 \\
\hline FGF Signaling & 3.98E-02 & 6.82E-02 & $\begin{array}{l}\text { FGFR3, FGF16, FGF18, FGFR1, FGF12, } \\
\text { CREB5 }\end{array}$ \\
\hline G-Protein Coupled Receptor Signaling & 4.37E-02 & 4.89E-02 & $\begin{array}{l}\text { PRKACB, SRC, GPR17, PDE2A, HTR2B, } \\
\text { PDE7B, DUSP1, PDE3A, TBXA2R, HTR1D, } \\
\text { PDE4B, PDE4D, CREB5 }\end{array}$ \\
\hline Cardiac $\beta$-adrenergic Signaling & 4.47E-02 & 5.33E-02 & $\begin{array}{l}\text { PRKACB, AKAP12, PDE2A, PDE7B, PDE3A, } \\
\text { PDE4B, PDE4D, PKIG }\end{array}$ \\
\hline Aryl Hydrocarbon Receptor Signaling & 4.57E-02 & 5.03E-02 & $\begin{array}{l}\text { SRC, NCOA7, CCND3, NFIA, RARB, } \\
\text { ALDH6A1, FAS, MCM7 }\end{array}$ \\
\hline ERK/MAPK Signaling & 4.68E-02 & 5.03E-02 & $\begin{array}{l}\text { PRKACB, ETS1, SRC, ELF4, H3F3A/H3F3B, } \\
\text { YWHAH, DUSP1, PLA2G4C, CREB5, STAT1 }\end{array}$ \\
\hline \multicolumn{4}{|l|}{ B. Non-reversal v Control } \\
\hline $\begin{array}{l}\text { UDP-D-xylose and UDP-D-glucuronate } \\
\text { Biosynthesis }\end{array}$ & $5.13 E-03$ & 1.43E-01 & UGDH \\
\hline Sphingomyelin Metabolism & 1.51E-02 & 7.14E-02 & SGMS2 \\
\hline VDR/RXR Activation & $1.70 \mathrm{E}-02$ & 2.47E-02 & MXD1, THBD \\
\hline G-Protein Coupled Receptor Signaling & $2.45 \mathrm{E}-02$ & 1.13E-02 & HTR2B, DUSP1, PDE4D \\
\hline $\begin{array}{l}\text { Hematopoiesis from Multipotent Stem } \\
\text { Cells }\end{array}$ & 3.02E-02 & 8.33E-02 & KITLG \\
\hline Colanic Acid Building Blocks Biosynthesis & 3.02E-02 & 2.94E-02 & UGDH \\
\hline p38 MAPK Signaling & 3.09E-02 & $1.75 \mathrm{E}-02$ & DUSP1, PLA2G4C \\
\hline Leukotriene Biosynthesis & 3.31E-02 & 4E-02 & MGST2 \\
\hline The Visual Cycle & $3.55 \mathrm{E}-02$ & 3.7E-02 & DHRS3 \\
\hline Glutathione Redox Reactions I & 3.80E-02 & $4.55 \mathrm{E}-02$ & MGST2 \\
\hline CDP-diacylglycerol Biosynthesis I & 3.80E-02 & 3.7E-02 & AGPAT9 \\
\hline Extrinsic Prothrombin Activation Pathway & 4.07E-02 & 5E-02 & THBD \\
\hline $\begin{array}{l}\text { Phosphatidylglycerol Biosynthesis II (Non- } \\
\text { plastidic) }\end{array}$ & 4.07E-02 & 3.03E-02 & AGPAT9 \\
\hline Aldosterone Signaling in Epithelial Cells & 4.90E-02 & 1.27E-02 & DUSP1, DNAJB9 \\
\hline
\end{tabular}


Chapter 6: Thoughts on the Direction of Future Research Projects

Joshua K Meisner 


\section{Summary}

While the studies in each chapter target somewhat disparate subjects, they all center on an overall theme of understanding the various elements that entail a successful adaptation to systemic arteriolar occlusive disease. This common purpose and goal constitutes my overall objective for future research. Given the primary origins of arterial occlusive disease (aging and lifestyle) are not likely to disappear, I believe understanding how these elements work together to compensate for the disease presents the best avenue for figuring out treatments for patients whose natural compensation is inadequate when that disease inevitably occurs. The results from each of the individual chapters suggest a variety of avenues of future pursuit, each of which may help further this endeavor. However, the results from each study also hint at a common conclusion: the adaptive response to ischemia is a very complex process, where any number of elements can fail, and this complexity requires analyzing those failures from multiple perspectives.

Each chapter highlights a different perspective from which to proceed toward better understanding successful adaptive responses to ischemia.

\section{Thoughts from Chapter 1}

As discussed in chapter 1 , it is clear that bone marrow derived cells play a central role in the arteriogenic pathway, but how the various populations interact with each other and the arteriogenic process is poorly understood. By understanding how these cell populations work together, we might be able to identify the BMC populations that are critical to either initiating or developing the arteriogenic process. Two approaches may help to answer these questions. First, obtaining a temporal resolution map of BMCs recruited during the process could be done using high-throughput techniques like DNA microarrays or RNA sequencing to look for lineage markers of immune cell populations in developing collateral arteries. This would allow for 
constructing a high resolution map of sequential recruitment. Such an approach produced some of the most interesting findings in previous studies using microarrays for transcriptional profiling of developing collateral arteries (21). With the lowered cost to microarrays and increased sensitivity of sequencing, these methods could be coupled with bioinformatics approaches to potentially produce an interact-ome of BMCs during collateral growth. Second, use of transgenic techniques for targeted ablation of various immune cell populations during femoral artery ligation may finally answer which specific BMC lineages are required for initiation and/or progression of arteriogenesis. This could be done through an approach such as crossing mice containing an inducible diphtheria toxin receptor (291) with transgenic mice with validated immune cell lineage-specific cre expression (e.g. neutrophil, macrophage).

\section{Thoughts from Chapter 2}

As discussed in chapter 2, the skeletal muscle myopathy present in PAD $(292,293)$ likely plays a key role in sustaining the perfusion deficit seen in response to ischemia. While these pathological changes occurring with PAD are due to the ischemia present with the disease, our data suggest that a shift in skeletal muscle regeneration can propagate these perfusion deficits. As such, there is potential in using factors that promote skeletal muscle regeneration and metabolic normalization in tandem with those that increase vascular remodeling. Going forward, using a multi-prong approach would likely yield the fastest progress toward that goal. For example, identifying candidate factors for preventing muscle myopathy during hypoxia or ischemia could be coupled with the development of targeted delivery methods pioneered in our laboratory (294) along with a careful selection/trial of vascular remodeling factors from the wide pool of previous studies. From there a combination of pre-selected candidates for correcting ischemia induced myopathy and enhancing vascular remodeling (or those identified in other aims) could be used in tandem to demonstrate enhanced reperfusion and functional recovery in the setting of hindlimb arterial occlusion. An additional question also arises from the data in 
chapter 2: does a pre-disposition toward ischemia-induced muscle pathology predict symptomatic progression of PAD within a segment of patients? To answer this question, it would be interesting to determine if muscle tissue from non-diseased limbs in symptomatic versus asymptomatic PAD patients or healthy controls show increasing sensitivity to ischemia/hypoxia in a method similar to that used previously to identify differences within diseased tissue (295). If there is a pre-disposed muscle phenotype that exacerbates PAD, that mechanism may serve as a unique starting point for an entirely new class of PAD treatments.

\section{Thoughts from Chapter 3}

While the preliminary data from chapter 3 suggests that angiogenesis and arteriogenesis each provide a significant contribution to the revascularization response, the data needs to be further strengthened. In the immediate future (ongoing), measurements of angiogenesis at an earlier time point are needed to confirm the hypothesis that an angiogenic deficit is the source of the early impairment of the reperfusion response. Further along, though, as discussed in chapter 3, identifying additional models that can differentially dissect the contributions of arteriogenesis and angiogenesis and their effect on subsequent reperfusion in both directions (i.e. including impaired arteriogenesis but not angiogenesis) would substantially strengthened the argument currently put forth. As discussed, multiple models likely exist for both cases, and there is a need to validate the arguments posed in chapter 3 to help bridge the divide within the therapeutic revascularization field. One particular strength going forward is that given the discrete test cases (impaired arteriogenesis but not angiogenesis, impaired angiogenesis but not arteriogenesis, or a combination of both), the project lends itself toward testable independent aims for each hypothesis within a larger, cohesive story within a future project proposal. 


\section{Thoughts from Chapter 4}

As discussed in chapter 4, continued development of tools that can interrogate dynamic changes in hemodynamics across vascular networks at the level of a single arteriole is necessary for fully understanding how hemodynamics guides microvascular structure. However, we demonstrated that the relatively straightforward technique of laser speckle flowmetry can produce useful and quantitative measurements of microvascular network hemodynamics. Going forward, I believe that further tool development of LSF would only yield marginal results. Instead, our demonstration of its usefulness in quantitative in vivo studies pushes the future direction of this project toward the application of LSF in vivo. This would harness the power of the current technique, while the tools that will surpass the capabilities of LSF (e.g. photoacoustic tomography) become more widely available. One such application is that utilized in chapter 5, where LSF was used to quantify the hemodynamic changes that initiate arteriogenesis along the length of a developing collateral artery. Other applications could be using the technique in a less invasive model than that used in chapter 4 to assess how changes in the hemodynamics of the microvasculature lead to BMC recruitment. In less-invasive models, it would be possible test the fundamental question of whether shear stress-induced BMC recruitment occurs at post-capillary venules and requires paired veins. Such an observation could have important implications for using multi-factor therapies to develop arterial supply into previously avascular regions (i.e. is venulogenesis necessary before arteriogenesis, or are capillaries alone sufficient guides, as suggested by Lucitti et al. (296)).

\section{Thoughts from Chapter 5}

The concept that directional change in shear stress produces a sustained enhancement of arteriogenesis opens numerous pathways for investigation aside from the potential implications to endothelial cell mechanotransduction experiments performed in vitro. Here I will 
focus on three central questions arising from that concept that could have significant impact on our understanding of adaptive arteriogenesis and, more broadly, vascular structural maintenance.

First, does this spatial enhancement of collateral development occur in humans? This is a critical question for determining the fundamental relevance of our observations. One approach to answer this may be looking at well-developed collateral arteries in patients with peripheral arterial occlusions to see if there is spatial enhancement in the signatures of arteriogenesis (i.e. is there increased tortuosity or lumenal diameter within regions of the arterial tree that suggest reversed flow direction prior to occlusion). However, there are three central complications with this approach. First, there is significant heterogeneity in the vascular tree of the lower limbs, where PAD predominantly occurs, making predicted directional changes more difficult. Second, the time-line of collateral development is unknown in established disease. So if the reversal enhancement dissipates over time, these spatial differences would likely be hard to detect. Third, endothelial cell function in PAD is often pathological, which could interfere with the reversal stimulus in an unpredictable way. However, if a reversal signature is present even with these complications, it would be a powerful argument for its presence in humans. A second approach could be to look at serial angiograms in stroke patients, where collateral architecture should be more consistent than in the peripheral vasculature and could potentially capture the arteriogenic process from an early time point.

Second, how is the reversal of shear direction enhancing the arteriogenic signaling process? One potential mechanism discussed in chapter 5 is that the pathways governing cytoskeletal remodeling may cross-talk with the arteriogenic response to increased shear stress. Perhaps more intriguing is the hypothesis that the cytoskeletal remodeling process is a required step to halt the arteriogenesis program. It may be that the primary response of endothelial cells to shear stress is to alter their cytoskeleton such that they reach an equilibrium point that 
minimizes drag force and the signaling cascade derived from altered shear stress. The process that provides the most logical method to obtain this state is through cytoskeletal remodeling by directly reducing the sensitivity to the force applied by new shear stress level and allowing the cell to return to quiescence. Such an adjustment likely requires much less remodeling and duration to adjust to a new shear regime in the same direction. At the same time, our observations in chapter 5 suggest the signaling cascades that conduct this cytoskeletal remodeling may coincidentally activate proliferation and inflammation pathways along the arteriogenic/remodeling pathway. Therefore, by prolonging this cytoskeletal remodeling, reversal increases the duration of these initiating pathways, yielding a stronger initial growth and inflammation response that are sufficient to generate and sustain the arteriogenic process. The subsequent recruitment of BMCs populations and activation of smooth muscle cells and adventitial cells is then able to propagate the growth response until negative feedback loops return the whole vessel to quiescence. Once the collateral has developed and the paracrine signaling from BMCs and SMC synthetic activity subsides, the maturation phase begins. During maturation, however, the sharp changes in hemodynamics are no longer present to initiate the remodeling cascade and vessel regression to normalize the spatial differences in diameter along the collateral artery.

There are several methods that might provide insight into each of these pathways. First, it would be essential to further mine the initial dataset presented in chapter 5 to identify a key marker or set of markers that denote a remodeling state versus a quiescent state (e.g. relative NFKB activation and KLF2 expression). It would then be possible to test if stabilizing the cytoskeleton to prevent adjustment to the new shear stress regime could prolong the remodeling response to increased shear stress. This could either be done with or without reversal, though reversal may provide a larger starting signal for teasing out differences. For the second hypothesis, testing if the pathways mediating cytoskeletal remodeling synergizes with 
the arteriogenic response pathway could be accomplished in a similar method. For example, one could over-express or knock down the key small GTPase pathway(s) that mediate the cytoskeletal remodeling process then determine how the markers of remodeling and quiescence are affected in response to constant or increased shear stress. These data could help explain the means by which endothelial cells detect the need to adjust the shear stress set point and then stabilize that new set point.

Third, how can we use this improved understanding of the mechanical stimuli driving arteriogenesis to therapeutic advantage? This question is difficult to really evaluate at this point in time. However, further mining of the arteriogenic signaling pathway may identify key regulators of the response. Additionally, by better understanding the mechanism of this process for moving and maintaining shear stress set points may provide good targets both for improving collateral development, but also for stabilizing early atherosclerotic plaque progression (e.g. would inhibiting small GTPase cross-talk raise the threshold for triggering the remodeling response?). Additionally, there may be therapeutic treatments that could enhance collateral development via recruiting collateral arteries in a reversed direction. For example, in the case of PAD where atherosclerotic lesions have not fully occluded, perhaps prolonged low-level exercise (below significant pain) or counter-pulsation therapy to increase venous return could create enough arterial inflow to generate a pressure drop at flow-limiting atherosclerotic occlusions. This would likely recruit collateral pathways bypassing the lesion against their normal direction of blood flow. The prolonged flow reversal may then act as a sufficient stimulus to reach the threshold to initiate robust collateral development.

In total, these chapters all prompt interesting questions that could be used toward the creation of new and exciting research directions for advancing the treatment of PAD and improved our understanding of vascular remodeling. 


\section{References}

1. Carmeliet P, Jain RK. Angiogenesis in cancer and other diseases. Nature. 2000; 407:249-57.

2. Schaper W. Collateral circulation: past and present. Basic Res Cardiol. 2009; 104:5-21.

3. Ziegler M a, Distasi MR, Bills RG, Miller SJ, Alloosh M, Murphy MP, George Akingba A, Sturek M, Dalsing MC, Unthank JL. Marvels, mysteries, and misconceptions of vascular compensation to peripheral artery occlusion. Microcirculation. 2010; 17:3-20.

4. Chilian WM, Penn MS, Pung YF, Dong F, Mayorga M, Ohanyan V, Logan S, Yin L. Coronary collateral growth--back to the future. J Mol Cell Cardiol. 2012; 52:905-11.

5. Troidl K, Schaper W. Arteriogenesis versus angiogenesis in peripheral artery disease. Diabetes Metab Res Rev. 2012; 28 Suppl 1:27-9.

6. Van Royen N, Piek JJ, Schaper W, Fulton WF. A critical review of clinical arteriogenesis research. J Am Coll Cardiol. 2009; 55:17-25.

7. Schirmer SH, Van Nooijen FC, Piek JJ, Van Royen N. Stimulation of collateral artery growth: travelling further down the road to clinical application. Heart. 2009; 95:191-7.

8. Norgren L, Hiatt WR, Dormandy JA, Nehler MR, Harris KA, Fowkes FGR. Inter-Society Consensus for the Management of Peripheral Arterial Disease (TASC II). J Vasc Surg. 2007; 45 Suppl S:S5-67.

9. Hirsch AT, Hartman L, Town RJ, Virnig BA. National health care costs of peripheral arterial disease in the Medicare population. Vasc Med. 2008; 13:209-15.

10. Kinnaird T, Stabile E, Burnett MS, Epstein SE. Bone-marrow-derived cells for enhancing collateral development: mechanisms, animal data, and initial clinical experiences. Circ Res. 2004; 95:354-63.

11. Kinnaird T, Stabile E, Zbinden S, Burnett MS, Epstein SE. Cardiovascular risk factors impair native collateral development and may impair efficacy of therapeutic interventions. Cardiovasc Res. 2008; 78:257-64.

12. Van Royen N, Schirmer SH, Atasever B, Behrens CYH, Ubbink D, Buschmann EE, Voskuil M, Bot P, Hoefer IE, Schlingemann RO, Biemond BJ, Tijssen JG, Bode C, Schaper W, Oskam J, Legemate DA, Piek JJ, Buschmann IR. START Trial: a pilot study on STimulation of ARTeriogenesis using subcutaneous application of granulocytemacrophage colony-stimulating factor as a new treatment for peripheral vascular disease. Circulation. 2005; 112:1040-6.

13. Van Weel V, Van Tongeren RB, Van Hinsbergh VWM, Van Bockel JH, Quax PH a. Vascular growth in ischemic limbs: a review of mechanisms and possible therapeutic stimulation. Ann Vasc Surg. 2008; 22:582-97. 
14. Schaper J, König R, Franz D, Schaper W. The endothelial surface of growing coronary collateral arteries. Intimal margination and diapedesis of monocytes. A combined SEM and TEM study. Virchows Arch A Pathol Anat Histol. 1976; 370:193-205.

15. Seiler C, Pohl T, Wustmann K, Hutter D, Nicolet PA, Windecker S, Eberli FR, Meier B. Promotion of collateral growth by granulocyte-macrophage colony-stimulating factor in patients with coronary artery disease: a randomized, double-blind, placebo-controlled study. Circulation. 2001; 104:2012-7.

16. Ince H, Petzsch M, Kleine HD, Eckard H, Rehders T, Burska D, Kische S, Freund M, Nienaber CA. Prevention of left ventricular remodeling with granulocyte colonystimulating factor after acute myocardial infarction: final 1-year results of the FrontIntegrated Revascularization and Stem Cell Liberation in Evolving Acute Myocardial Infarction by Gran. Circulation. 2005; 112:I73-80.

17. Ripa RS, Jørgensen E, Wang Y, Thune JJ, Nilsson JC, Søndergaard L, Johnsen HE, Køber L, Grande P, Kastrup J. Stem cell mobilization induced by subcutaneous granulocyte-colony stimulating factor to improve cardiac regeneration after acute STelevation myocardial infarction: result of the double-blind, randomized, placebo-controlled stem cells in myocardial infarc. Circulation. 2006; 113:1983-92.

18. Zohlnhöfer D, Ott I, Mehilli J, Schömig K, Michalk F, Ibrahim T, Meisetschläger G, Von Wedel J, Bollwein H, Seyfarth M, Dirschinger J, Schmitt C, Schwaiger M, Kastrati A, Schömig A. Stem cell mobilization by granulocyte colony-stimulating factor in patients with acute myocardial infarction: a randomized controlled trial. JAMA. 2006; 295:100310.

19. Heil M, Schaper W. Influence of mechanical, cellular, and molecular factors on collateral artery growth (arteriogenesis). Circ Res. 2004; 95:449-58.

20. Deindl E, Buschmann IR, Hoefer IE, Podzuweit T, Boengler K, Vogel S, Van Royen N, Fernandez B, Schaper W. Role of ischemia and of hypoxia-inducible genes in arteriogenesis after femoral artery occlusion in the rabbit. Circ Res. 2001; 89:779-86.

21. Lee CW, Stabile E, Kinnaird T, Shou M, Devaney JM, Epstein SE, Burnett MS. Temporal patterns of gene expression after acute hindlimb ischemia in mice: insights into the genomic program for collateral vessel development. J Am Coll Cardiol. 2004; 43:474-82.

22. Yang Y, Tang G, Yan J, Park B, Hoffman A, Tie G, Wang R, Messina LM. Cellular and molecular mechanism regulating blood flow recovery in acute versus gradual femoral artery occlusion are distinct in the mouse. J Vasc Surg. 2008; 48:1546-58.

23. Troidl K, Tribulova S, Cai W-J, Rüding I, Apfelbeck H, Schierling W, Troidl C, SchmitzRixen T, Schaper W. Effects of endogenous nitric oxide and of DETA NONOate in arteriogenesis. J Cardiovasc Pharmacol. 2010; 55:153-60.

24. Pipp F, Boehm S, Cai W-J, Adili F, Ziegler B, Karanovic G, Ritter R, Balzer J, Scheler C, Schaper W, Schmitz-Rixen T. Elevated fluid shear stress enhances postocclusive collateral artery growth and gene expression in the pig hind limb. Arterioscler Thromb Vasc Biol. 2004; 24:1664-8. 
25. Eitenmüller IK, Volger O, Kluge A, Troidl K, Barancik M, Cai W-J, Heil M, Pipp F, Fischer S, Horrevoets AJG, Schmitz-Rixen T, Schaper W. The range of adaptation by collateral vessels after femoral artery occlusion. Circ Res. 2006; 99:656-62.

26. Hershey JC, Baskin EP, Glass JD, Hartman HA, Gilberto DB, Rogers IT, Cook JJ. Revascularization in the rabbit hindlimb: dissociation between capillary sprouting and arteriogenesis. Cardiovasc Res. 2001; 49:618-25.

27. Ito WD. The role of monocytes/macrophages and vascular resident precursor cells in collateral growth. In: Deindl E, Kupatt C, editors. Therapeutic Neovascularization-Quo Vadis? Springer; 2007. p. 227-255.

28. Lash JM, Nixon JC, Unthank JL. Exercise training effects on collateral and microvascular resistances in rat model of arterial insufficiency. Am J Physiol. 1995; 268:H125-37.

29. Unthank JL, Nixon JC, Lash JM. Early adaptations in collateral and microvascular resistances after ligation of the rat femoral artery. J Appl Physiol. 1995; 79:73-82.

30. Yun J, Rocic P, Pung YF, Belmadani S, Carrao ACR, Ohanyan V, Chilian WM. Redoxdependent mechanisms in coronary collateral growth: the "redox window" hypothesis. Antioxid Redox Signal. 2009; 11:1961-74.

31. Gregory AD, Capoccia BJ, Woloszynek JR, Link DC. Systemic levels of G-CSF and interleukin- 6 determine the angiogenic potential of bone marrow resident monocytes. $J$ Leukoc Biol. 2010; 88:123-31.

32. Scholz D, Ito WD, Fleming I, Deindl E, Sauer A, Wiesnet M, Busse R, Schaper J, Schaper W. Ultrastructure and molecular histology of rabbit hind-limb collateral artery growth (arteriogenesis). Virchows Arch. 2000; 436:257-70.

33. Hoefer IE, Van Royen N, Rectenwald JE, Deindl E, Hua J, Jost MM, Grundmann S, Voskuil M, Ozaki CK, Piek JJ, Buschmann IR. Arteriogenesis proceeds via ICAM-1/Mac1- mediated mechanisms. Circ Res. 2004; 94:1179-85.

34. Shireman PK. The chemokine system in arteriogenesis and hind limb ischemia. $J$ Vasc Surg. 2007; 45 Suppl A:A48-56.

35. Wolf C, Cai W-J, Vosschulte R, Koltai S, Mousavipour D, Scholz D, Afsah-Hedjri A, Schaper W, Schaper J. Vascular remodeling and altered protein expression during growth of coronary collateral arteries. J Mol Cell Cardiol. 1998; 30:2291-305.

36. Cai W-J, Kocsis E, Wu X, Rodríguez M, Luo X, Schaper W, Schaper J. Remodeling of the vascular tunica media is essential for development of collateral vessels in the canine heart. Mol Cell Biochem. 2004; 264:201-10.

37. Cai W-J, Koltai S, Kocsis E, Scholz D, Kostin S, Luo X, Schaper W, Schaper J. Remodeling of the adventitia during coronary arteriogenesis. Am J Physiol Heart Circ Physiol. 2003; 284:H31-40. 
38. Cai W, Vosschulte R, Afsah-Hedjri A, Koltai S, Kocsis E, Scholz D, Kostin S, Schaper W, Schaper J. Altered balance between extracellular proteolysis and antiproteolysis is associated with adaptive coronary arteriogenesis. J Mol Cell Cardiol. 2000; 32:997-1011.

39. Feaver RE, Gelfand BD, Wang C, Schwartz MA, Blackman BR. Atheroprone hemodynamics regulate fibronectin deposition to create positive feedback that sustains endothelial inflammation. Circ Res. 2010; 106:1703-11.

40. Gagne PJ, Tihonov N, Li X, Glaser J, Qiao J, Silberstein M, Yee H, Gagne E, Brooks P. Temporal exposure of cryptic collagen epitopes within ischemic muscle during hindlimb reperfusion. Am J Pathol. 2005; 167:1349-59.

41. Hobeika MJ, Edlin RS, Muhs BE, Sadek M, Gagne PJ. Matrix metalloproteinases in critical limb ischemia. J Surg Res. 2008; 149:148-54.

42. Lee S, Jilani SM, Nikolova G V, Carpizo D, Iruela-Arispe ML. Processing of VEGF-A by matrix metalloproteinases regulates bioavailability and vascular patterning in tumors. $J$ Cell Biol. 2005; 169:681-91.

43. Kinnaird T, Stabile E, Burnett MS, Shou M, Lee CW, Barr S, Fuchs S, Epstein SE. Local delivery of marrow-derived stromal cells augments collateral perfusion through paracrine mechanisms. Circulation. 2004; 109:1543-9.

44. Ziegelhoeffer T, Fernandez B, Kostin S, Heil M, Voswinckel R, Helisch A, Schaper W. Bone marrow-derived cells do not incorporate into the adult growing vasculature. Circ Res. 2004; 94:230-8.

45. Khmelewski E, Becker A, Meinertz T, Ito WD. Tissue resident cells play a dominant role in arteriogenesis and concomitant macrophage accumulation. Circ Res. 2004; 95:E5664.

46. Arras M, Ito WD, Scholz D, Winkler B, Schaper J, Schaper W. Monocyte activation in angiogenesis and collateral growth in the rabbit hindlimb. J Clin Invest. 1998; 101:40-50.

47. Schaper W, Scholz D. Factors regulating arteriogenesis. Arterioscler Thromb Vasc Biol. 2003; 23:1143-51.

48. Bakker ENTP, Matlung HL, Bonta P, De Vries CJ, Van Rooijen N, Van Bavel E. Blood flow-dependent arterial remodelling is facilitated by inflammation but directed by vascular tone. Cardiovasc Res. 2008; 78:341-8.

49. Andrade-Silva AR, Ramalho FS, Ramalho LNZ, Saavedra-Lopes M, Jordão A a, Vanucchi H, Piccinato CE, Zucoloto S. Effect of NFkappaB inhibition by CAPE on skeletal muscle ischemia-reperfusion injury. J Surg Res. 2009; 153:254-62.

50. Tidball JG. Inflammatory cell response to acute muscle injury. Med Sci Sports Exerc. 1995; 27:1022-32. 
51. Behm CZ, Kaufmann B a, Carr C, Lankford M, Sanders JM, Rose CE, Kaul S, Lindner JR. Molecular imaging of endothelial vascular cell adhesion molecule-1 expression and inflammatory cell recruitment during vasculogenesis and ischemia-mediated arteriogenesis. Circulation. 2008; 117:2902-11.

52. Hoefer IE, Grundmann S, Van Royen N, Voskuil M, Schirmer SH, Ulusans S, Bode C, Buschmann IR, Piek JJ. Leukocyte subpopulations and arteriogenesis: specific role of monocytes, lymphocytes and granulocytes. Atherosclerosis. 2005; 181:285-93.

53. Ohki Y, Heissig B, Sato Y, Akiyama H, Zhu Z, Hicklin DJ, Shimada K, Ogawa H, Daida H, Hattori K, Ohsaka A. Granulocyte colony-stimulating factor promotes neovascularization by releasing vascular endothelial growth factor from neutrophils. FASEB J. 2005; 19:2005-7.

54. Soehnlein O, Zernecke A, Eriksson EE, Rothfuchs AG, Pham CT, Herwald H, Bidzhekov K, Rottenberg ME, Weber C, Lindbom L. Neutrophil secretion products pave the way for inflammatory monocytes. Blood. 2008; 112:1461-1471.

55. Heissig B, Nishida C, Tashiro Y, Sato Y, Ishihara M, Ohki M, Gritli I, Rosenkvist J, Hattori K. Role of neutrophil-derived matrix metalloproteinase-9 in tissue regeneration. Histol Histopathol. 2010; 25:765-70.

56. Nozawa $\mathrm{H}$, Chiu C, Hanahan D. Infiltrating neutrophils mediate the initial angiogenic switch in a mouse model of multistage carcinogenesis. Proc Natl Acad Sci U S A. 2006; 103:12493-8.

57. Gong Y, Koh D-RR. Neutrophils promote inflammatory angiogenesis via release of preformed VEGF in an in vivo corneal model. Cell Tissue Res. 2010; 339:437-48.

58. Hao Q, Chen Y, Zhu Y, Fan Y, Palmer D, Su H, Young WL, Yang G-YY. Neutrophil depletion decreases VEGF-induced focal angiogenesis in the mature mouse brain. $J$ Cereb Blood Flow Metab. 2007; 27:1853-60.

59. Capoccia BJ, Shepherd RM, Link DC. G-CSF and AMD3100 mobilize monocytes into the blood that stimulate angiogenesis in vivo through a paracrine mechanism. Blood. 2006; 108:2438-45.

60. Couffinhal T, Silver M, Kearney M, Sullivan A, Witzenbichler B, Magner M, Annex B, Peters K, Isner JM. Impaired collateral vessel development associated with reduced expression of vascular endothelial growth factor in ApoE-/- mice. Circulation. 1999; 99:3188-98.

61. Stabile E, Burnett MS, Watkins C, Kinnaird T, Bachis A, La Sala A, Miller JM, Shou M, Epstein SE, Fuchs S. Impaired arteriogenic response to acute hindlimb ischemia in CD4knockout mice. Circulation. 2003; 108:205-10.

62. Van Weel V, Toes REM, Seghers L, Deckers MML, De Vries MR, Eilers PH, Sipkens J, Schepers A, Eefting D, Van Hinsbergh VWM, Van Bockel JH, Quax PH a. Natural killer cells and CD4+ T-cells modulate collateral artery development. Arterioscler Thromb Vasc Biol. 2007; 27:2310-8. 
63. Stabile E, Kinnaird T, La Sala A, Hanson SK, Watkins C, Campia U, Shou M, Zbinden S, Fuchs S, Kornfeld H, Epstein SE, Burnett MS. CD8+ T lymphocytes regulate the arteriogenic response to ischemia by infiltrating the site of collateral vessel development and recruiting CD4+ mononuclear cells through the expression of interleukin-16. Circulation. 2006; 113:118-24.

64. Willems S. The potential role of monocyte-lymphocyte interaction in arteriogenesis. Angiogenesis. 2009;

65. Schaper W. Prevention of tissue death by killer cells? The role of the immune system in arteriogenesis. Arterioscler Thromb Vasc Biol. 2007; 27:2273-4.

66. Colucci F, Caligiuri M a, Di Santo JP. What does it take to make a natural killer? Nat Rev Immunol. 2003; 3:413-25.

67. Mac Gabhann F, Peirce SM, Gabhann M. Collateral capillary arterialization following arteriolar ligation in murine skeletal muscle. Microcirculation. 2010; 17:333-47.

68. Zhang $\mathrm{H}$, Prabhakar $\mathrm{P}$, Sealock $\mathrm{R}$, Faber JE. Wide genetic variation in the native pial collateral circulation is a major determinant of variation in severity of stroke. $J$ Cereb Blood Flow Metab. 2010; 30:923-34.

69. Taherzadeh Z, Van Bavel E, De Vos J, Matlung HL, Van Montfrans G, Brewster LM, Seghers L, Quax PH a, Bakker ENTP. Strain-dependent susceptibility for hypertension in mice resides in the natural killer gene complex. Am J Physiol Heart Circ Physiol. 2010; 298:H1273-82.

70. Sun J, Sukhova GK, Wolters PJ, Yang M, Kitamoto S, Libby P, MacFarlane LA, Mallen-St Clair J, Shi G-P. Mast cells promote atherosclerosis by releasing proinflammatory cytokines. Nat Med. 2007; 13:719-24.

71. Kelley JL, Chi DS, bou-Auda W, Smith JK, Krishnaswamy G, Abou-Auda W. The molecular role of mast cells in atherosclerotic cardiovascular disease. Mol Med Today. 2000; 6:304-8.

72. Heissig B, Rafii S, Akiyama H, Ohki Y, Sato Y, Rafael T, Zhu Z, Hicklin DJ, Okumura K, Ogawa H, Werb Z, Hattori K. Low-dose irradiation promotes tissue revascularization through VEGF release from mast cells and MMP-9-mediated progenitor cell mobilization. $J$ Exp Med. 2005; 202:739-50.

73. Schramm R, Thorlacius $H$. Neutrophil recruitment in mast cell-dependent inflammation: inhibitory mechanisms of glucocorticoids. Inflamm Res. 2004; 53:644-52.

74. Ryu J, Lee CW, Hong K-H, Shin J-A, Lim S-H, Park C-S, Shim J, Nam KB, Choi K-J, Kim $\mathrm{Y}-\mathrm{H}$, Han $\mathrm{KH}$. Activation of fractalkine/CX3CR1 by vascular endothelial cells induces angiogenesis through VEGF-A/KDR and reverses hindlimb ischaemia. Cardiovasc Res. 2008; 78:333-40. 
75. Papadopoulos EJ, Fitzhugh DJ, Tkaczyk C, Gilfillan AM, Sassetti C, Metcalfe DD, Hwang ST. Mast cells migrate, but do not degranulate, in response to fractalkine, a membranebound chemokine expressed constitutively in diverse cells of the skin. Eur J Immunol. 2000; 30:2355-61.

76. El-Shazly A, Berger P, Girodet P-OO, Ousova O, Fayon M, Vernejoux J-MM, Marthan R, Tunon-de-Lara JM. Fraktalkine produced by airway smooth muscle cells contributes to mast cell recruitment in asthma. $J$ Immunol. 2006; 176:1860-8.

77. Bergmann CE, Hoefer IE, Meder B, Roth H, Van Royen N, Breit SM, Jost MM, Aharinejad S, Hartmann S, Buschmann IR. Arteriogenesis depends on circulating monocytes and macrophage accumulation and is severely depressed in op/op mice. J Leukoc Biol. 2006; 80:59-65.

78. Heil M, Ziegelhoeffer T, Pipp F, Kostin S, Martin S, Clauss M, Schaper W. Blood monocyte concentration is critical for enhancement of collateral artery growth. $A m J$ Physiol Heart Circ Physiol. 2002; 283:H2411-9.

79. Pipp F, Heil M, Issbrücker K, Ziegelhoeffer T, Martin S, Van den Heuvel J, Weich H, Fernandez B, Golomb G, Carmeliet P, Schaper W, Clauss M. VEGFR-1-selective VEGF homologue PIGF is arteriogenic: evidence for a monocyte-mediated mechanism. Circ Res. 2003; 92:378-85.

80. Capoccia BJ, Gregory AD, Link DC. Recruitment of the inflammatory subset of monocytes to sites of ischemia induces angiogenesis in a monocyte chemoattractant protein-1-dependent fashion. J Leukoc Biol. 2008; 84:760-8.

81. Contreras-Shannon V, Ochoa O, Reyes-Reyna SM, Sun D, Michalek JE, Kuziel WA, McManus LM, Shireman PK. Fat accumulation with altered inflammation and regeneration in skeletal muscle of CCR2-/- mice following ischemic injury. Am J Physiol Cell Physiol. 2007; 292:C953-67.

82. Geissmann F, Jung S, Littman DR. Blood monocytes consist of two principal subsets with distinct migratory properties. Immunity. 2003; 19:71-82.

83. Gordon S, Taylor PR. Monocyte and macrophage heterogeneity. Nat Rev Immunol. 2005; 5:953-64.

84. Swirski FK, Weissleder R, Pittet MJ. Heterogeneous in vivo behavior of monocyte subsets in atherosclerosis. Arterioscler Thromb Vasc Biol. 2009; 29:1424-32.

85. Woollard KJ, Geissmann F. Monocytes in atherosclerosis: subsets and functions. Nat Rev Cardiol. 2010; 7:77-86.

86. Epstein SE, Stabile E, Kinnaird T, Lee CW, Clavijo L, Burnett MS. Janus phenomenon: the interrelated tradeoffs inherent in therapies designed to enhance collateral formation and those designed to inhibit atherogenesis. Circulation. 2004; 109:2826-31. 
87. Swirski FK, Nahrendorf M, Etzrodt M, Wildgruber M, Cortez-Retamozo V, Panizzi P, Figueiredo J-L, Kohler RH, Chudnovskiy A, Waterman P, Aikawa E, Mempel TR, Libby P, Weissleder R, Pittet MJ. Identification of splenic reservoir monocytes and their deployment to inflammatory sites. Science. 2009; 325:612-6.

88. Auffray C, Fogg D, Garfa M, Elain G, Join-Lambert O, Kayal S, Sarnacki S, Cumano A, Lauvau G, Geissmann F. Monitoring of blood vessels and tissues by a population of monocytes with patrolling behavior. Science. 2007; 317:666-70.

89. Meisner JK, Song J, Price RJ. Arteriolar and Venular Remodeling Are Differentially Regulated by Bone Marrow-Derived Cell-Specific CX3CR1 and CCR2 Expression. PLoS ONE. 2012; 7:e46312.

90. Nickerson MM, Song J, Meisner JK, Bajikar S, Burke CW, Shuptrine CW, Owens GK, Skalak TC, Price RJ. Bone marrow-derived cell-specific chemokine (C-C motif) receptor-2 expression is required for arteriolar remodeling. Arterioscler Thromb Vasc Biol. 2009; 29:1794-801.

91. Asahara T, Murohara T, Sullivan A, Silver M, Van der Zee R, Li T, Witzenbichler B, Schatteman GC, Isner JM. Isolation of Putative Progenitor Endothelial Cells for Angiogenesis. Science (80- ). 1997; 275:964-966.

92. Kirton JP, Xu Q. Endothelial precursors in vascular repair. Microvasc Res. 2010; 79:1939.

93. Lian Q, Zhang Y, Zhang J, Zhang HK, Wu X, Zhang Y, Lam FF-Y, Kang S, Xia JC, Lai W$\mathrm{H}$, Au K-W, Chow YY, Siu C-W, Lee C-N, Tse H-F. Functional mesenchymal stem cells derived from human induced pluripotent stem cells attenuate limb ischemia in mice. Circulation. 2010; 121:1113-23.

94. Zampetaki A, Kirton JP, Xu Q. Vascular repair by endothelial progenitor cells. Cardiovasc Res. 2008; 78:413-21.

95. Yoon C-H, Hur J, Park K-W, Kim J-H, Lee C-S, Oh I-Y, Kim T-Y, Cho H-J, Kang H-J, Chae I-H, Yang H-K, Oh B-H, Park Y-B, Kim H-S. Synergistic neovascularization by mixed transplantation of early endothelial progenitor cells and late outgrowth endothelial cells: the role of angiogenic cytokines and matrix metalloproteinases. Circulation. 2005; 112:1618-27.

96. Hoenig MR, Bianchi C, Sellke FW. Hypoxia inducible factor-1 alpha, endothelial progenitor cells, monocytes, cardiovascular risk, wound healing, cobalt and hydralazine: a unifying hypothesis. Curr Drug Targets. 2008; 9:422-35.

97. Hur J, Yoon C-H, Kim H-S, Choi J-H, Kang H-J, Hwang K-K, Oh B-H, Lee M-M, Park Y-B. Characterization of two types of endothelial progenitor cells and their different contributions to neovasculogenesis. Arterioscler Thromb Vasc Biol. 2004; 24:288-93.

98. Hur J, Yang H-M, Yoon C-H, Lee C-S, Park K-W, Kim J-HJ-Y, Kim T-Y, Kang H-J, Chae $\mathrm{I}-\mathrm{H}$, Oh $\mathrm{B}-\mathrm{H}$, Park $\mathrm{Y}-\mathrm{B}$, Kim $\mathrm{H}-\mathrm{S}$. Identification of a novel role of $\mathrm{T}$ cells in postnatal 
vasculogenesis: characterization of endothelial progenitor cell colonies. Circulation. 2007; 116:1671-1682.

99. Nickerson MM, Burke CW, Meisner JK, Shuptrine CW, Song J, Price RJ. Capillary arterialization requires the bone-marrow-derived cell (BMC)-specific expression of chemokine ( $\mathrm{C}-\mathrm{C}$ motif) receptor-2, but BMCs do not transdifferentiate into microvascular smooth muscle. Angiogenesis. 2009; 12:355-63.

100. O'Neill TJ, Wamhoff BR, Owens GK, Skalak TC. Mobilization of bone marrow-derived cells enhances the angiogenic response to hypoxia without transdifferentiation into endothelial cells. Circ Res. 2005; 97:1027-35.

101. Kinnaird T, Stabile E, Burnett MS, Lee CW, Barr S, Fuchs S, Epstein SE. Marrow-derived stromal cells express genes encoding a broad spectrum of arteriogenic cytokines and promote in vitro and in vivo arteriogenesis through paracrine mechanisms. Circ Res. 2004; 94:678-85.

102. Ito WD, Khmelewski E, Khmelevski E. Tissue macrophages: "satellite cells" for growing collateral vessels? A hypothesis. Endothelium. 2003; 10:233-5.

103. Buschmann IR, Hoefer IE. All arteriogenesis is local? Home boys versus the newcomers. Circ Res. 2004; 95:e72.

104. Corselli M, Chen C-W, Crisan M, Lazzari L, Péault B. Perivascular ancestors of adult multipotent stem cells. Arterioscler Thromb Vasc Biol. 2010; 30:1104-9.

105. Arnold L, Henry A, Poron F, Baba-Amer Y, Van Rooijen N, Plonquet A, Gherardi RK, Chazaud B. Inflammatory monocytes recruited after skeletal muscle injury switch into antiinflammatory macrophages to support myogenesis. J Exp Med. 2007; 204:1057-69.

106. Hester RL, Hammer LW. Venular-arteriolar communication in the regulation of blood flow. Am J Physiol Regul Integr Comp Physiol. 2002; 282:R1280-5.

107. Boegehold MA. Shear-dependent release of venular nitric oxide: effect on arteriolar tone in rat striated muscle. Am J Physiol. 1996; 271:H387-95.

108. Nellore K, Harris NR. Nitric oxide measurements in rat mesentery reveal disrupted venulo-arteriolar communication in diabetes. Microcirculation. 2004; 11:415-23.

109. Kavdia M, Popel AS. Venular endothelium-derived NO can affect paired arteriole: a computational model. Am J Physiol Heart Circ Physiol. 2006; 290:H716-23.

110. Matlung HL, Bakker ENTP, Van Bavel E. Shear stress, reactive oxygen species, and arterial structure and function. Antioxid Redox Signal. 2009; 11:1699-709.

111. Zbinden S, Wang J, Adenika R, Schmidt M, Tilan JU, Najafi AH, Peng X, LassanceSoares RM, lantorno M, Morsli H, Gercenshtein L, Jang GJ, Epstein SE, Burnett MS. Metallothionein enhances angiogenesis and arteriogenesis by modulating smooth muscle cell and macrophage function. Arterioscler Thromb Vasc Biol. 2010; 30:477-82. 
112. Dai X, Faber JE. Endothelial nitric oxide synthase deficiency causes collateral vessel rarefaction and impairs activation of a cell cycle gene network during arteriogenesis. Circ Res. 2010; 106:1870-81.

113. De Lussanet QG, Van Golde JCG, Beets-Tan RG, De Haan MW, Zaar DVJ, Post MJ, Huijberts MS, Schaper NC, Van Engelshoven JM a, Backes WH. Magnetic resonance angiography of collateral vessel growth in a rabbit femoral artery ligation model. NMR Biomed. 2006; 19:77-83.

114. Rockstroh J, Brown BG. Coronary collateral size, flow capacity, and growth: estimates from the angiogram in patients with obstructive coronary disease. Circulation. 2002; 105:168-73.

115. Sefcik LS, Wilson JL, Papin J a, Botchwey E a. Harnessing systems biology approaches to engineer functional microvascular networks. Tissue Eng Part B Rev. 2010; 16:361-70.

116. Helisch A, Wagner S, Khan N, Drinane M, Wolfram S, Heil M, Ziegelhoeffer T, Brandt U, Pearlman JD, Swartz HM, Schaper W. Impact of mouse strain differences in innate hindlimb collateral vasculature. Arterioscler Thromb Vasc Biol. 2006; 26:520-6.

117. Benest A V, Stone OA, Miller WH, Glover CP, Uney JB, Baker AH, Harper SJ, Bates DO. Arteriolar genesis and angiogenesis induced by endothelial nitric oxide synthase overexpression results in a mature vasculature. Arterioscler Thromb Vasc Biol. 2008; 28:1462-8.

118. Johnstone MT, Creager SJ, Scales KM, Cusco JA, Lee BK, Creager MA. Impaired endothelium-dependent vasodilation in patients with insulin-dependent diabetes mellitus. Circulation. 1993; 88:2510-6.

119. Rivard A, Fabre J-E, Silver M, Chen D, Murohara T, Kearney M, Magner M, Asahara T, Isner JM. Age-dependent impairment of angiogenesis. Circulation. 1999; 99:111-20.

120. Zhou X, Bohlen HG, Miller SJ, Unthank JL. NAD(P)H oxidase-derived peroxide mediates elevated basal and impaired flow-induced NO production in SHR mesenteric arteries in vivo. Am J Physiol Heart Circ Physiol. 2008; 295:H1008-H1016.

121. Zhou X, Bohlen HG, Unthank JL, Miller SJ. Abnormal nitric oxide production in aged rat mesenteric arteries is mediated by $\mathrm{NAD}(\mathrm{P}) \mathrm{H}$ oxidase-derived peroxide. Am J Physiol Heart Circ Physiol. 2009; 297:H2227-33.

122. Murohara T, Asahara T, Silver M, Bauters C, Masuda H, Kalka C, Kearney M, Chen D, Symes JF, Fishman MC, Huang PL, Isner JM. Nitric oxide synthase modulates angiogenesis in response to tissue ischemia. J Clin Invest. 1998; 101:2567-78.

123. Heil M, Ziegelhoeffer T, Wagner S, Fernandez B, Helisch A, Martin S, Tribulova S, Kuziel WA, Bachmann G, Schaper W. Collateral artery growth (arteriogenesis) after experimental arterial occlusion is impaired in mice lacking CC-chemokine receptor-2. Circ Res. 2004; 94:671-7. 
124. Waltenberger $\mathrm{J}$, Lange $\mathrm{J}$, Kranz A. Vascular endothelial growth factor-A-induced chemotaxis of monocytes is attenuated in patients with diabetes mellitus: A potential predictor for the individual capacity to develop collaterals. Circulation. 2000; 102:185-90.

125. Heeschen C, Lehmann R, Honold J, Assmus B, Aicher A, Walter DH, Martin H, Zeiher AM, Dimmeler S. Profoundly reduced neovascularization capacity of bone marrow mononuclear cells derived from patients with chronic ischemic heart disease. Circulation. 2004; 109:1615-22.

126. Schultz A, Lavie L, Hochberg I, Beyar R, Stone T, Skorecki K, Lavie P, Roguin A, Levy AP. Interindividual heterogeneity in the hypoxic regulation of VEGF: significance for the development of the coronary artery collateral circulation. Circulation. 1999; 100:547-52.

127. Chittenden TW, Sherman JA, Xiong F, Hall AE, Lanahan AA, Taylor JM, Duan H, Pearlman JD, Moore JH, Schwartz SM, Simons M. Transcriptional profiling in coronary artery disease: indications for novel markers of coronary collateralization. Circulation. 2006; 114:1811-20.

128. Chalothorn D, Zhang H, Smith JE, Edwards JC, Faber JE. Chloride intracellular channel4 is a determinant of native collateral formation in skeletal muscle and brain. Circ Res. 2009 ; 105:89-98.

129. Chalothorn D, Faber JE. Formation and maturation of the native cerebral collateral circulation. J Mol Cell Cardiol. 2010; 49:251-9.

130. Clayton JA, Chalothorn D, Faber JE. Vascular endothelial growth factor-A specifies formation of native collaterals and regulates collateral growth in ischemia. Circ Res. 2008; 103:1027-36.

131. Sonntag WE, Lynch CD, Cooney PT, Hutchins PM. Decreases in cerebral microvasculature with age are associated with the decline in growth hormone and insulinlike growth factor 1. Endocrinology. 1997; 138:3515-20.

132. Qian HS, De Resende MM, Beausejour C, Huw L-YY, Liu P, Rubanyi GM, Kauser K. Age-dependent acceleration of ischemic injury in endothelial nitric oxide synthasedeficient mice: potential role of impaired VEGF receptor 2 expression. J Cardiovasc Pharmacol. 2006; 47:587-93.

133. Amann B, Lüdemann C, Rückert R, Lawall H, Liesenfeld B, Schneider M, Schmidt-Lucke $\mathrm{J}$. Design and rationale of a randomized, double-blind, placebo-controlled phase III study for autologous bone marrow cell transplantation in critical limb ischemia: the BONe Marrow Outcomes Trial in Critical Limb Ischemia (BONMOT-CLI). VASA. Zeitschrift für Gefässkrankheiten. Journal for vascular diseases. 2008; 37:319-25.

134. Sprengers RW, Moll FL, Teraa M, Verhaar MC. Rationale and design of the JUVENTAS trial for repeated intra-arterial infusion of autologous bone marrow-derived mononuclear cells in patients with critical limb ischemia. J Vasc Surg. 2010; 51:1564-8.

135. Lawall H, Bramlage P, Amann B. Stem cell and progenitor cell therapy in peripheral artery disease. A critical appraisal. Thromb Haemost. 2010; 103:696-709. 
136. Van Oostrom MC, Van Oostrom O, Quax PH a, Verhaar MC, Hoefer IE. Insights into mechanisms behind arteriogenesis: what does the future hold? J Leukoc Biol. 2008; 84:1379-91.

137. Vartanian SM, Sarkar R. Therapeutic angiogenesis. Vasc Endovascular Surg. 2006; 41:173-85.

138. Carmeliet P. Angiogenesis in health and disease. Nat Med. 2003; 9:653-60.

139. Robbins JL, Jones WS, Duscha BD, Allen JD, Kraus WE, Regensteiner JG, Hiatt WR, Annex $\mathrm{BH}$. Relationship between leg muscle capillary density and peak hyperemic blood flow with endurance capacity in peripheral artery disease. J Appl Physiol. 2011; 111:816.

140. McClung JM, McCord TJ, Keum S, Johnson S, Annex BH, Marchuk D a, Kontos CD. Skeletal muscle-specific genetic determinants contribute to the differential straindependent effects of hindlimb ischemia in mice. Am J Pathol. 2012; 180:2156-69.

141. Chalothorn D, Clayton JA, Zhang H, Pomp D, Faber JE. Collateral density, remodeling, and VEGF-A expression differ widely between mouse strains. Physiol Genomics. 2007; 30:179-91.

142. Scholz D, Ziegelhoeffer T, Helisch A, Wagner S, Friedrich C, Podzuweit T, Schaper W. Contribution of arteriogenesis and angiogenesis to postocclusive hindlimb perfusion in mice. J Mol Cell Cardiol. 2002; 34:775-87.

143. Johnson C, Sung H-J, Lessner SM, Fini ME, Galis ZS. Matrix metalloproteinase-9 is required for adequate angiogenic revascularization of ischemic tissues: potential role in capillary branching. Circ Res. 2004; 94:262-8.

144. Muhs BE, Gagne PJ, Plitas G, Shaw JP, Shamamian P. Experimental hindlimb ischemia leads to neutrophil-mediated increases in gastrocnemius MMP-2 and -9 activity: a potential mechanism for ischemia induced MMP activation. J Surg Res. 2004; 117:249_ 54.

145. Jin DK, Shido K, Kopp H-G, Petit I, Shmelkov S V, Young LM, Hooper AT, Amano H, Avecilla ST, Heissig B, Hattori K, Zhang F, Hicklin DJ, Wu Y, Zhu Z, Dunn A, Salari H, Werb Z, Hackett NR, Crystal RG, Lyden D, Rafii S. Cytokine-mediated deployment of SDF-1 induces revascularization through recruitment of CXCR4+ hemangiocytes. Nat Med. 2006; 12:557-67.

146. Huang P-H, Chen Y-H, Wang C-H, Chen J-SJ-W, Tsai H-Y, Lin F-Y, Lo W-Y, Wu T-C, Sata M, Lin S-J. Matrix metalloproteinase-9 is essential for ischemia-induced neovascularization by modulating bone marrow-derived endothelial progenitor cells. Arterioscler Thromb Vasc Biol. 2009; 29:1179-84.

147. Haas TL, Doyle JL, Distasi MR, Norton LE, Sheridan KM, Unthank JL. Involvement of MMPs in the outward remodeling of collateral mesenteric arteries. Am J Physiol Heart Circ Physiol. 2007; 293:H2429-37. 
148. Plitas G, Gagne PJ, Muhs BE, lanus IA, Shaw JP, Beudjekian M, Delgado Y, Jacobowitz G, Rockman C, Shamamian P. Experimental hindlimb ischemia increases neutrophilmediated matrix metalloproteinase activity: a potential mechanism for lung injury after limb ischemia• 1. J Am Coll Surg. 2003; 196:761-767.

149. Jönsson S, Lundberg A, Kälvegren H, Bergström I, Szymanowski A, Jonasson L. Increased levels of leukocyte-derived MMP-9 in patients with stable angina pectoris. PLoS One. 2011; 6:e19340.

150. Ochoa O, Sun D, Reyes-Reyna SM, Waite LL, Michalek JE, McManus LM, Shireman PK. Delayed angiogenesis and VEGF production in CCR2-/- mice during impaired skeletal muscle regeneration. Am J Physiol Regul Integr Comp Physiol. 2007; 293:R651-61.

151. Meisner JK, Price RJ. Spatial and temporal coordination of bone marrow-derived cell activity during arteriogenesis: regulation of the endogenous response and therapeutic implications. Microcirculation. 2010; 17:583-99.

152. Chen X, Li Y. Role of matrix metalloproteinases in skeletal muscle: migration, differentiation, regeneration and fibrosis. Cell Adh Migr. 2009; 3:337-41.

153. Heissig B, Hattori K, Dias S, Friedrich M, Ferris B, Hackett NR, Crystal RG, Besmer P, Lyden D, Moore MASS, Werb Z, Rafii S. Recruitment of stem and progenitor cells from the bone marrow niche requires MMP-9 mediated release of kit-ligand. Cell. 2002; 109:625-37.

154. Chappell JC, Song J, Klibanov AL, Price RJ. Ultrasonic microbubble destruction stimulates therapeutic arteriogenesis via the CD18-dependent recruitment of bone marrow-derived cells. Arterioscler Thromb Vasc Biol. 2008; 28:1117-22.

155. Distasi MR, Case J, Ziegler M a, Dinauer MC, Yoder MC, Haneline LS, Dalsing MC, Miller SJ, Labarrere C a, Murphy MP, Ingram D a, Unthank JL. Suppressed hindlimb perfusion in Rac2-/- and Nox2-/- mice does not result from impaired collateral growth. Am J Physiol Heart Circ Physiol. 2009; 296:H877-86.

156. Schindelin J, Arganda-Carreras I, Frise E, Kaynig V, Longair M, Pietzsch T, Preibisch S, Rueden C, Saalfeld S, Schmid B, Tinevez J-Y, White DJ, Hartenstein V, Eliceiri K, Tomancak P, Cardona A. Fiji: an open-source platform for biological-image analysis. Nat Methods. 2012; 9:676-82.

157. Suzuki J, Kobayashi T, Uruma T, Koyama T. Strength training with partial ischaemia stimulates microvascular remodelling in rat calf muscles. Eur J Appl Physiol. 2000; 82:215-22.

158. Burkholder TJ, Fingado B, Baron S, Lieber RL. Relationship between muscle fiber types and sizes and muscle architectural properties in the mouse hindlimb. $J$ Morphol. 1994; 221:177-90.

159. Schiaffino S, Reggiani C. Fiber types in mammalian skeletal muscles. Physiol Rev. 2011; 91:1447-531. 
160. Chalothorn D, Zhang H, Clayton JA, Thomas SA, Faber JE. Catecholamines augment collateral vessel growth and angiogenesis in hindlimb ischemia. Am J Physiol Heart Circ Physiol. 2005; 289:H947-59.

161. Muhs BE, Plitas G, Delgado $Y$, lanus IA, Shaw JP, Adelman MA, Lamparello P, Shamamian P, Gagne PJ. Temporal expression and activation of matrix metalloproteinases-2, -9 , and membrane type 1-matrix metalloproteinase following acute hindlimb ischemia. J Surg Res. 2003; 111:8-15.

162. Hudlicka O, Brown M, Egginton S. Angiogenesis in skeletal and cardiac muscle. Physiol Rev. 1992; 72:369-417.

163. Shireman PK, Quinones MP. Differential necrosis despite similar perfusion in mouse strains after ischemia. J Surg Res. 2005; 129:242-50.

164. Tidball JG. Inflammatory processes in muscle injury and repair. Am J Physiol Regul Integr Comp Physiol. 2005; 288:R345-53.

165. Kherif S, Lafuma C, Dehaupas M, Lachkar S, Fournier JG, Verdière-Sahuqué M, Fardeau $\mathrm{M}$, Alameddine HS. Expression of matrix metalloproteinases 2 and 9 in regenerating skeletal muscle: a study in experimentally injured and mdx muscles. Dev Biol. 1999; 205:158-70.

166. Fukushima K, Nakamura A, Ueda H, Yuasa K, Yoshida K, Takeda S, Ikeda S. Activation and localization of matrix metalloproteinase-2 and -9 in the skeletal muscle of the muscular dystrophy dog (CXMDJ). BMC Musculoskelet Disord. 2007; 8:54.

167. Yeghiazaryan M, Żybura-Broda K, Cabaj A, Włodarczyk J, Sławińska U, Rylski M, Wilczyński GM. Fine-structural distribution of MMP-2 and MMP-9 activities in the rat skeletal muscle upon training: a study by high-resolution in situ zymography. Histochem Cell Biol. 2012; 138:75-87.

168. Yu Q, Stamenkovic I. Cell surface-localized matrix metalloproteinase-9 proteolytically activates TGF-beta and promotes tumor invasion and angiogenesis. Genes Dev. 2000; 14:163-76.

169. Hao Q, Su H, Palmer D, Sun B, Gao P, Yang G-Y, Young WL. Bone marrow-derived cells contribute to vascular endothelial growth factor-induced angiogenesis in the adult mouse brain by supplying matrix metalloproteinase-9. Stroke. 2011; 42:453-8.

170. Gargioli C, Coletta M, De Grandis F, Cannata SM, Cossu G. PIGF-MMP-9-expressing cells restore microcirculation and efficacy of cell therapy in aged dystrophic muscle. Nat Med. 2008; 14:973-8.

171. Han J-K, Kim H-L, Jeon K-H, Choi Y-E, Lee H-S, Kwon Y-W, Jang J-J, Cho H-J, Kang HJ, Oh B-H, Park Y-B, Kim H-S. Peroxisome proliferator-activated receptor-\{delta\} activates endothelial progenitor cells to induce angio-myogenesis through matrix metalloproteinase-9-mediated insulin-like growth factor-1 paracrine networks. Eur Heart J. 2011;:ehr365-. 
172. Ryu JC, Davidson BP, Xie A, Qi Y, Zha D, Belcik JT, Caplan ES, Woda JM, Hedrick CC, Hanna RN, Lehman N, Zhao Y, Ting A, Lindner JR. Molecular imaging of the paracrine proangiogenic effects of progenitor cell therapy in limb ischemia. Circulation. 2013; 127:710-9.

173. Tirziu D, Jaba IM, Yu P, Larrivée B, Coon BG, Cristofaro B, Zhuang ZW, Lanahan AA, Schwartz MA, Eichmann A, Simons M. Endothelial nuclear factor-kB-dependent regulation of arteriogenesis and branching. Circulation. 2012; 126:2589-600.

174. Skuli N, Majmundar AJ, Krock BL, Mesquita RC, Mathew LK, Quinn ZL, Runge A, Liu L, Kim MN, Liang J, Schenkel S, Yodh AG, Keith B, Simon MC. Endothelial HIF-2a regulates murine pathological angiogenesis and revascularization processes. $J$ Clin Invest. 2012; 122:1427-43.

175. Cristofaro B, Shi Y, Faria M, Suchting S, Leroyer AS, Trindade A, Duarte A, Zovein AC, Iruela-Arispe ML, Nih LR, Kubis N, Henrion D, Loufrani L, Todiras M, Schleifenbaum J, Gollasch M, Zhuang ZW, Simons M, Eichmann A, Le Noble F. DIl4-Notch signaling determines the formation of native arterial collateral networks and arterial function in mouse ischemia models. Development. 2013; 140:1720-9.

176. Tayebjee MH, Tan KT, MacFadyen RJ, Lip GYH. Abnormal circulating levels of metalloprotease 9 and its tissue inhibitor 1 in angiographically proven peripheral arterial disease: relationship to disease severity. J Intern Med. 2005; 257:110-6.

177. Creager MA, Olin JW, Belch JJF, Moneta GL, Henry TD, Rajagopalan S, Annex BH, Hiatt WR. Effect of hypoxia-inducible factor-1alpha gene therapy on walking performance in patients with intermittent claudication. Circulation. 2011; 124:1765-73.

178. Kastrup J, Jørgensen E, Rück A, Tägil K, Glogar D, Ruzyllo W, Bøtker HE, Dudek D, Drvota V, Hesse B, Thuesen L, Blomberg P, Gyöngyösi M, Sylvén C. Direct intramyocardial plasmid vascular endothelial growth factor-A165 gene therapy in patients with stable severe angina pectoris A randomized double-blind placebo-controlled study: the Euroinject One trial. J Am Coll Cardiol. 2005; 45:982-8.

179. Rajagopalan S, Mohler ER, Lederman RJ, Mendelsohn FO, Saucedo JF, Goldman CK, Blebea J, Macko J, Kessler PD, Rasmussen HS, Annex BH. Regional angiogenesis with vascular endothelial growth factor in peripheral arterial disease: a phase II randomized, double-blind, controlled study of adenoviral delivery of vascular endothelial growth factor 121 in patients with disabling intermittent cl. Circulation. 2003; 108:1933-8.

180. Subramaniyam V, Waller EK, Murrow JR, Manatunga A, Lonial S, Kasirajan K, Sutcliffe D, Harris W, Taylor WR, Alexander RW, Quyyumi AA. Bone marrow mobilization with granulocyte macrophage colony-stimulating factor improves endothelial dysfunction and exercise capacity in patients with peripheral arterial disease. Am Heart J. 2009; 158:5360.e1.

181. Simons M, Annex BH, Laham RJ, Kleiman N, Henry T, Dauerman H, Udelson JE, Gervino E V, Pike M, Whitehouse MJ, Moon T, Chronos NA. Pharmacological treatment of coronary artery disease with recombinant fibroblast growth factor-2: double-blind, randomized, controlled clinical trial. Circulation. 2002; 105:788-93. 
182. Kusumanto $\mathrm{YH}$, Van Weel V, Mulder NH, Smit AJ, Van den Dungen JJAM, Hooymans JMM, Sluiter WJ, Tio RA, Quax PHA, Gans ROB, Dullaart RPF, Hospers GAP. Treatment with intramuscular vascular endothelial growth factor gene compared with placebo for patients with diabetes mellitus and critical limb ischemia: a double-blind randomized trial. Hum Gene Ther. 2006; 17:683-91.

183. West a. M, Anderson JD, Epstein FH, Meyer $\mathrm{CH}$, Hagspiel KD, Berr SS, Harthun NL, Weltman a. L, Annex $\mathrm{BH}$, Kramer CM. Percutaneous intervention in peripheral artery disease improves calf muscle phosphocreatine recovery kinetics: A pilot study. Vascular Medicine. 2012; 17:3-9.

184. Hazarika S, Angelo M, Li Y, Aldrich AJ, Odronic SI, Yan Z, Stamler JS, Annex BH. Myocyte specific overexpression of myoglobin impairs angiogenesis after hind-limb ischemia. Arterioscler Thromb Vasc Biol. 2008; 28:2144-50.

185. Flögel U, Merx MW, Godecke A, Decking UK, Schrader J. Myoglobin: A scavenger of bioactive NO. Proc Natl Acad Sci U S A. 2001; 98:735-40.

186. Ziche M, Morbidelli L, Masini E, Amerini S, Granger HJ, Maggi CA, Geppetti P, Ledda F. Nitric oxide mediates angiogenesis in vivo and endothelial cell growth and migration in vitro promoted by substance P. J Clin Invest. 1994; 94:2036-44.

187. Thomas DD, Liu X, Kantrow SP, Lancaster JR. The biological lifetime of nitric oxide: implications for the perivascular dynamics of $\mathrm{NO}$ and O2. Proc Natl Acad Sci U S A. 2001; 98:355-60.

188. Kavdia M, Tsoukias NM, Popel AS. Model of nitric oxide diffusion in an arteriole: impact of hemoglobin-based blood substitutes. Am J Physiol Heart Circ Physiol. 2002; 282:H2245-53.

189. Yan Z, Serrano AL, Schiaffino S, Bassel-Duby R, Williams RS. Regulatory elements governing transcription in specialized myofiber subtypes. J Biol Chem. 2001; 276:173616.

190. Schuenke MD, Kopchick JJ, Hikida RS, Kraemer WJ, Staron RS. Effects of growth hormone overexpression vs. growth hormone receptor gene disruption on mouse hindlimb muscle fiber type composition. Growth Horm IGF Res. 2008; 18:479-86.

191. Kanatous SB, Mammen PPA, Rosenberg PB, Martin CM, White MD, Dimaio JM, Huang G, Muallem S, Garry DJ. Hypoxia reprograms calcium signaling and regulates myoglobin expression. Am J Physiol Cell Physiol. 2009; 296:C393-402.

192. Grange RW, Meeson A, Chin E, Lau KS, Stull JT, Shelton JM, Williams RS, Garry DJ. Functional and molecular adaptations in skeletal muscle of myoglobin-mutant mice. $A m \mathrm{~J}$ Physiol Cell Physiol. 2001; 281:C1487-1494.

193. Van Weel V, Deckers MML, Grimbergen JM, Van Leuven KJM, Lardenoye JHP, Schlingemann RO, Van Nieuw Amerongen GP, Van Bockel JH, Van Hinsbergh VWM, Quax PHA. Vascular endothelial growth factor overexpression in ischemic skeletal muscle enhances myoglobin expression in vivo. Circ Res. 2004; 95:58-66. 
194. De P, Peng Q, Traktuev DO, Traktuevc DO, Li W, Yoder MC, March KL, Durden DL. Expression of RAC2 in endothelial cells is required for the postnatal neovascular response. Exp Cell Res. 2009; 315:248-63.

195. Wu Y, Ip JE, Huang J, Zhang L, Matsushita K, Liew C-C, Pratt RE, Dzau VJ. Essential role of ICAM-1/CD18 in mediating EPC recruitment, angiogenesis, and repair to the infarcted myocardium. Circ Res. 2006; 99:315-22.

196. Yoon C-H, Hur J, Oh I-Y, Park K-W, Kim T-Y, Shin J-H, Kim J-H, Lee C-S, Chung J-K, Park Y-B, Kim H-S. Intercellular adhesion molecule-1 is upregulated in ischemic muscle, which mediates trafficking of endothelial progenitor cells. Arterioscler Thromb Vasc Biol. 2006; 26:1066-72.

197. Tongers J, Roncalli JG, Losordo DW. Role of endothelial progenitor cells during ischemia-induced vasculogenesis and collateral formation. Microvasc Res. 2010; 79:2006.

198. Anderson JL, Halperin JL, Albert NM, Bozkurt B, Brindis RG, Curtis LH, DeMets D, Guyton RA, Hochman JS, Kovacs RJ, Ohman EM, Pressler SJ, Sellke FW, Shen W-K. Management of Patients With Peripheral Artery Disease (Compilation of 2005 and 2011 ACCF/AHA Guideline Recommendations): A Report of the American College of Cardiology Foundation/American Heart Association Task Force on Practice Guidelines. Circulation. 2013; 127:1425-1443.

199. Duscha BD, Robbins JL, Jones WS, Kraus WE, Lye RJ, Sanders JM, Allen JD, Regensteiner JG, Hiatt WR, Annex BH. Angiogenesis in skeletal muscle precede improvements in peak oxygen uptake in peripheral artery disease patients. Arterioscler Thromb Vasc Biol. 2011; 31:2742-8.

200. Popel AS, Johnson PC. Microcirculation and Hemorheology. Annu Rev Fluid Mech. 2005; 37:43-69.

201. Pries AR, Secomb TW. Control of blood vessel structure: insights from theoretical models. Am J Physiol Heart Circ Physiol. 2005; 288:H1010-5.

202. Pries AR, Secomb TW. Modeling structural adaptation of microcirculation. Microcirculation. 2008; 15:753-64.

203. Fercher AF, Briers JD. Flow visualization by means of single-exposure speckle photography. Optics Communications. 1981; 37:326-330.

204. Draijer M, Hondebrink E, Van Leeuwen T, Steenbergen W. Review of laser speckle contrast techniques for visualizing tissue perfusion. Lasers Med Sci. 2009; 24:639-51.

205. Boas D a, Dunn AK. Laser speckle contrast imaging in biomedical optics. J Biomed Opt. 2010; 15:011109. 
206. Ramirez-San-Juan JC, Ramos-García R, Guizar-Iturbide I, Martínez-Niconoff G, Choi B. Impact of velocity distribution assumption on simplified laser speckle imaging equation. Opt Express. 2008; 16:3197-203.

207. Cheng H, Luo Q, Zeng S, Chen S, Cen J, Gong H. Modified laser speckle imaging method with improved spatial resolution. J Biomed Opt. 2003; 8:559-64.

208. Yang O, Cuccia D, Choi B. Real-time blood flow visualization using the graphics processing unit. J Biomed Opt. 2011; 16:016009.

209. Strong AJ, Bezzina EL, Anderson PJB, Boutelle MG, Hopwood SE, Dunn AK. Evaluation of laser speckle flowmetry for imaging cortical perfusion in experimental stroke studies: quantitation of perfusion and detection of peri-infarct depolarisations. J Cereb Blood Flow Metab. 2006; 26:645-53.

210. Armitage GA, Todd KG, Shuaib A, Winship IR. Laser speckle contrast imaging of collateral blood flow during acute ischemic stroke. J Cereb Blood Flow Metab. 2010; $30: 1432-6$.

211. Dunn AK, Bolay $H$, Moskowitz MA, Boas DA. Dynamic imaging of cerebral blood flow using laser speckle. J Cereb Blood Flow Metab. 2001; 21:195-201.

212. Ayata C, Dunn AK, Gursoy-OZdemir Y, Huang Z, Boas DA, Moskowitz MA. Laser speckle flowmetry for the study of cerebrovascular physiology in normal and ischemic mouse cortex. J Cereb Blood Flow Metab. 2004; 24:744-55.

213. Cheng $\mathrm{H}$, Luo $\mathrm{Q}$, Liu $\mathrm{Q}$, Lu $\mathrm{Q}$, Gong $\mathrm{H}$, Zeng $\mathrm{S}$. Laser speckle imaging of blood flow in microcirculation. Phys Med Biol. 2004; 49:1347-57.

214. Choi B, Ramirez-San-Juan JC, Lotfi J, Stuart Nelson J. Linear response range characterization and in vivo application of laser speckle imaging of blood flow dynamics. $J$ Biomed Opt. 2006; 11:41129.

215. White SM, George SC, Choi B. Automated computation of functional vascular density using laser speckle imaging in a rodent window chamber model. Microvasc Res. 2011; 82:92-5.

216. Nagahara M, Tamaki $Y$, Tomidokoro A, Araie M. In vivo measurement of blood velocity in human major retinal vessels using the laser speckle method. Invest Ophthalmol Vis Sci. 2011; 52:87-92.

217. Rege A, Thakor $\mathrm{N} \mathrm{V}$, Rhie $\mathrm{K}$, Pathak AP. In vivo laser speckle imaging reveals microvascular remodeling and hemodynamic changes during wound healing angiogenesis. Angiogenesis. 2012; 15:87-98.

218. Duncan DD, Kirkpatrick SJ. Can laser speckle flowmetry be made a quantitative tool? $J$ Opt Soc Am A Opt Image Sci Vis. 2008; 25:2088-94. 
219. Baez S. An open cremaster muscle preparation for the study of blood vessels by in vivo microscopy. Microvasc Res. 1973; 5:384-94.

220. Kirkpatrick SJ, Duncan DD, Wells-Gray EM. Detrimental effects of speckle-pixel size matching in laser speckle contrast imaging. Opt Lett. 2008; 33:2886-8.

221. Baker M, Wayland $\mathrm{H}$. On-line volume flow rate and velocity profile measurement for blood in microvessels. Microvasc Res. 1974; 7:131-43.

222. Ishikawa M, Sekizuka E, Shimizu K, Yamaguchi N, Kawase T. Measurement of RBC velocities in the rat pial arteries with an image-intensified high-speed video camera system. Microvasc Res. 1998; 56:166-72.

223. Soehle $M$, Heimann A, Kempski O. On the number of measurement sites required to assess regional cerebral blood flow by laser-Doppler scanning during cerebral ischemia and reperfusion. $J$ Neurosci Methods. 2001; 110:91-4.

224. Davis A, Izatt J, Rothenberg F. Quantitative measurement of blood flow dynamics in embryonic vasculature using spectral Doppler velocimetry. Anat Rec (Hoboken). 2009; 292:311-9.

225. Zhao Y, Chen Z, Saxer C, Xiang S, De Boer JF, Nelson JS. Phase-resolved optical coherence tomography and optical Doppler tomography for imaging blood flow in human skin with fast scanning speed and high velocity sensitivity. Opt Lett. 2000; 25:114-6.

226. Al-Khazraji BK, Novielli NM, Goldman D, Medeiros PJ, Jackson DN. A simple "streak length method" for quantifying and characterizing red blood cell velocity profiles and blood flow in rat skeletal muscle arterioles. Microcirculation. 2012; 19:327-35.

227. Smith ML, Long DS, Damiano ER, Ley K. Near-wall micro-PIV reveals a hydrodynamically relevant endothelial surface layer in venules in vivo. Biophys J. 2003; 85:637-45.

228. Nakano A, Sugii $Y$, Minamiyama M, Niimi H. Measurement of red cell velocity in microvessels using particle image velocimetry (PIV). Clin Hemorheol Microcirc. 2003; 29:445-55.

229. Kleinfeld D, Mitra PP, Helmchen F, Denk W. Fluctuations and stimulus-induced changes in blood flow observed in individual capillaries in layers 2 through 4 of rat neocortex. Proc Natl Acad Sci U S A. 1998; 95:15741-6.

230. Kamoun WS, Chae S-S, Lacorre DA, Tyrrell JA, Mitre M, Gillissen MA, Fukumura D, Jain RK, Munn LL. Simultaneous measurement of RBC velocity, flux, hematocrit and shear rate in vascular networks. Nat Methods. 2010; 7:655-60.

231. Borders JL, Granger HJ. An optical doppler intravital velocimeter. Microvasc Res. 1984; 27:117-27. 
232. Wayland $\mathrm{H}$, Johnson PC. Erythrocyte velocity measurement in microvessels by a two-slit photometric method. J Appl Physiol. 1967; 22:333-7.

233. Hu S, Wang L V. Photoacoustic imaging and characterization of the microvasculature. $J$ Biomed Opt. 2010; 15:011101.

234. Nguyen Q-T, Tsai PS, Kleinfeld D. MPScope: a versatile software suite for multiphoton microscopy. J Neurosci Methods. 2006; 156:351-9.

235. Nguyen J, Nishimura N, Fetcho RN, ladecola C, Schaffer CB. Occlusion of cortical ascending venules causes blood flow decreases, reversals in flow direction, and vessel dilation in upstream capillaries. J Cereb Blood Flow Metab. 2011; 31:2243-54.

236. Ryan MJ, Didion SP, Davis DR, Faraci FM, Sigmund CD. Endothelial dysfunction and blood pressure variability in selected inbred mouse strains. Arterioscler Thromb Vasc Biol. 2002; 22:42-8.

237. Leonard S, Croy BA, Murrant CL. Arteriolar reactivity in lymphocyte-deficient mice. Am J Physiol Heart Circ Physiol. 2011; 301:H1276-85.

238. Van Gieson EJ, Murfee WL, Skalak TC, Price RJ. Enhanced smooth muscle cell coverage of microvessels exposed to increased hemodynamic stresses in vivo. Circ Res. 2003; 92:929-36.

239. Tuttle JL, Nachreiner RD, Bhuller AS, Condict KW, Connors BA, Herring BP, Dalsing MC, Unthank JL. Shear level influences resistance artery remodeling: wall dimensions, cell density, and eNOS expression. Am J Physiol Heart Circ Physiol. 2001; 281:H1380-9.

240. Billaud M, Ross JA, Greyson MA, Bruce AC, Seaman SA, Heberlein KR, Han J, Best AK, Peirce SM, Isakson BE. A new method for in vivo visualization of vessel remodeling using a near-infrared dye. Microcirculation. 2011; 18:163-71.

241. Demicheva E, Hecker M, Korff T. Stretch-induced activation of the transcription factor activator protein-1 controls monocyte chemoattractant protein-1 expression during arteriogenesis. Circ Res. 2008; 103:477-84.

242. Skala MC, Fontanella A, Lan L, Izatt $\mathrm{J}$ a, Dewhirst MW. Longitudinal optical imaging of tumor metabolism and hemodynamics. J Biomed Opt. 2010; 15:011112.

243. Rentrop KP, Cohen $\mathrm{M}$, Blanke $\mathrm{H}$, Phillips RA. Changes in collateral channel filling immediately after controlled coronary artery occlusion by an angioplasty balloon in human subjects. J Am Coll Cardiol. 1985; 5:587-92.

244. Williams DO, Amsterdam EA, Miller RR, Mason DT. Functional significance of coronary collateral vessels in patients with acute myocardial infarction: relation to pump performance, cardiogenic shock and survival. Am J Cardiol. 1976; 37:345-51. 
245. Schierling W, Troidl K, Mueller C, Troidl C, Wustrack H, Bachmann G, Kasprzak PM, Schaper W, Schmitz-Rixen T. Increased intravascular flow rate triggers cerebral arteriogenesis. J Cereb Blood Flow Metab. 2009; 29:726-37.

246. Chiu J-J, Chien S. Effects of disturbed flow on vascular endothelium: pathophysiological basis and clinical perspectives. Physiol Rev. 2011; 91:327-87.

247. Wang L V, Hu S. Photoacoustic tomography: in vivo imaging from organelles to organs. Science. 2012; 335:1458-62.

248. Meisner JK, Sumer S, Murrell KP, Higgins TJ, Price RJ. Laser speckle flowmetry method for measuring spatial and temporal hemodynamic alterations throughout large microvascular networks. Microcirculation. 2012; 19:619-31.

249. Dunn JF, Forrester KR, Martin L, Tulip J, Bray RC. A transmissive laser speckle imaging technique for measuring deep tissue blood flow: an example application in finger joints. Lasers Surg Med. 2011; 43:21-8.

250. Parmar KM, Larman HB, Dai G, Zhang Y, Wang ET, Moorthy SN, Kratz JR, Lin Z, Jain MK, Gimbrone MA, García-Cardeña G. Integration of flow-dependent endothelial phenotypes by Kruppel-like factor 2. J Clin Invest. 2006; 116:49-58.

251. Ren B, Deng Y, Mukhopadhyay A, Lanahan AA, Zhuang ZW, Moodie KL, Mulligan-Kehoe MJ, Byzova $T$ V, Peterson RT, Simons M. ERK1/2-Akt1 crosstalk regulates arteriogenesis in mice and zebrafish. J Clin Invest. 2010; 120:1217-28.

252. Bir SC, Xiong Y, Kevil CG, Luo J. Emerging role of PKA/eNOS pathway in therapeutic angiogenesis for ischaemic tissue diseases. Cardiovasc Res. 2012; 95:7-18.

253. Venkatesh PK, Pattillo CB, Branch B, Hood J, Thoma S, Illum S, Pardue S, Teng X, Patel RP, Kevil CG. Dipyridamole enhances ischaemia-induced arteriogenesis through an endocrine nitrite/nitric oxide-dependent pathway. Cardiovasc Res. 2010; 85:661-70.

254. Tzima E, Irani-Tehrani M, Kiosses WB, Dejana E, Schultz DA, Engelhardt B, Cao G, DeLisser $\mathrm{H}$, Schwartz MA. A mechanosensory complex that mediates the endothelial cell response to fluid shear stress. Nature. 2005; 437:426-31.

255. Mack PJ, Zhang Y, Chung S, Vickerman V, Kamm RD, García-Cardeña G. Biomechanical Regulation of Endothelium-dependent Events Critical for Adaptive Remodeling. J Biol Chem. 2009; 284:8412-20.

256. Irizarry RA, Bolstad BM, Collin F, Cope LM, Hobbs B, Speed TP. Summaries of Affymetrix GeneChip probe level data. Nucleic Acids Res. 2003; 31:e15.

257. Chappell JC, Song J, Burke CW, Klibanov AL, Price RJ. Targeted delivery of nanoparticles bearing fibroblast growth factor-2 by ultrasonic microbubble destruction for therapeutic arteriogenesis. Small. 2008; 4:1769-77. 
258. Hode $T$, Jenkins $P$, Jordison $S$, Hode L. To what extent is coherence lost in tissue? In: Hamblin MR, Waynant RW, Anders J, editors. SPIE BiOS. 2011. p. 788703-788703-10.

259. McCue S, Dajnowiec D, Xu F, Zhang M, Jackson MR, Langille BL. Shear stress regulates forward and reverse planar cell polarity of vascular endothelium in vivo and in vitro. Circ Res. 2006; 98:939-46.

260. Tzima E, Kiosses WB, Del Pozo MA, Schwartz MA. Localized cdc42 activation, detected using a novel assay, mediates microtubule organizing center positioning in endothelial cells in response to fluid shear stress. J Biol Chem. 2003; 278:31020-3.

261. Rogers K a, McKee NH, Kalnins VI. Preferential orientation of centrioles toward the heart in endothelial cells of major blood vessels is reestablished after reversal of a segment. Proc Natl Acad Sci U S A. 1985; 82:3272-6.

262. Allan VJ, Thompson HM, McNiven MA. Motoring around the Golgi. Nat Cell Biol. 2002; 4:E236-42.

263. Coan DE, Wechezak a R, Viggers RF, Sauvage LR. Effect of shear stress upon localization of the Golgi apparatus and microtubule organizing center in isolated cultured endothelial cells. J Cell Sci. 1993; 104 ( Pt 4:1145-53.

264. Blackman BR, García-Cardeña G, Gimbrone MA. A new in vitro model to evaluate differential responses of endothelial cells to simulated arterial shear stress waveforms. $J$ Biomech Eng. 2002; 124:397-407.

265. Eden E, Navon R, Steinfeld I, Lipson D, Yakhini Z. GOrilla: a tool for discovery and visualization of enriched GO terms in ranked gene lists. BMC Bioinformatics. 2009; 10:48.

266. Garcia-Cardeña G, Comander J, Anderson KR, Blackman BR, Gimbrone M a. Biomechanical activation of vascular endothelium as a determinant of its functional phenotype. Proc Natl Acad Sci U S A. 2001; 98:4478-85.

267. Zhang J, Friedman $\mathrm{MH}$. Adaptive response of vascular endothelial cells to an acute increase in shear stress magnitude. Am J Physiol Heart Circ Physiol. 2012; 302:H98391.

268. Senthilkumar A, Smith RD, Khitha J, Arora N, Veerareddy S, Langston W, Chidlow JH, Barlow SC, Teng X, Patel RP, Lefer DJ, Kevil CG. Sildenafil promotes ischemia-induced angiogenesis through a PKG-dependent pathway. Arterioscler Thromb Vasc Biol. 2007; 27:1947-54.

269. Sarateanu CS, Retuerto MA, Beckmann JT, McGregor L, Carbray J, Patejunas G, Nayak L, Milbrandt J, Rosengart TK. An Egr-1 master switch for arteriogenesis: studies in Egr-1 homozygous negative and wild-type animals. J Thorac Cardiovasc Surg. 2006; 131:13845.

270. Buschmann I, Pries A, Styp-Rekowska B, Hillmeister P, Loufrani L, Henrion D, Shi Y, Duelsner A, Hoefer I, Gatzke N, Wang H, Lehmann K, Ulm L, Ritter Z, Hauff P, 
Hlushchuk R, Djonov V, Van Veen T, Le Noble F. Pulsatile shear and Gja5 modulate arterial identity and remodeling events during flow-driven arteriogenesis. Development. 2010; 137:2187-96.

271. Chen Z, Rubin J, Tzima E. Role of PECAM-1 in arteriogenesis and specification of preexisting collaterals. Circ Res. 2010; 107:1355-63.

272. Toyota E, Warltier DC, Brock T, Ritman E, Kolz C, O'Malley P, Rocic P, Focardi M, Chilian WM. Vascular endothelial growth factor is required for coronary collateral growth in the rat. Circulation. 2005; 112:2108-13.

273. Babiak A, Schumm A-M, Wangler C, Loukas M, Wu J, Dombrowski S, Matuschek C, Kotzerke J, Dehio C, Waltenberger J. Coordinated activation of VEGFR-1 and VEGFR-2 is a potent arteriogenic stimulus leading to enhancement of regional perfusion. Cardiovasc Res. 2004; 61:789-95.

274. Hayashi S -i., Morishita R, Nakamura S, Yamamoto K, Moriguchi A, Nagano T, Taiji M, Noguchi H, Matsumoto K, Nakamura T, Higaki J, Ogihara T. Potential Role of Hepatocyte Growth Factor, a Novel Angiogenic Growth Factor, in Peripheral Arterial Disease: Downregulation of HGF in Response to Hypoxia in Vascular Cells. Circulation. 1999; 100:II-301-II-308.

275. Taniyama Y, Morishita R, Aoki M, Nakagami H, Yamamoto K, Yamazaki K, Matsumoto K, Nakamura T, Kaneda Y, Ogihara T. Therapeutic angiogenesis induced by human hepatocyte growth factor gene in rat and rabbit hindlimb ischemia models: preclinical study for treatment of peripheral arterial disease. Gene Ther. 2001; 8:181-9.

276. Van Royen N, Hoefer I, Buschmann I, Heil M, Kostin S, Deindl E, Vogel S, Korff T, Augustin H, Bode C, Piek JJ, Schaper W. Exogenous application of transforming growth factor beta 1 stimulates arteriogenesis in the peripheral circulation. FASEB journal: official publication of the Federation of American Societies for Experimental Biology. 2002; 16:432-4.

277. Masaki I, Yonemitsu Y, Yamashita A, Sata S, Tanii M, Komori K, Nakagawa K, Hou X, Nagai $\mathrm{Y}$, Hasegawa M, Sugimachi K, Sueishi K. Angiogenic gene therapy for experimental critical limb ischemia: acceleration of limb loss by overexpression of vascular endothelial growth factor 165 but not of fibroblast growth factor-2. Circ Res. 2002; 90:966-73.

278. Fujii T, Yonemitsu Y, Onimaru M, Tanii M, Nakano T, Egashira K, Takehara T, Inoue M, Hasegawa M, Kuwano H, Sueishi K. Nonendothelial mesenchymal cell-derived MCP-1 is required for FGF-2-mediated therapeutic neovascularization: critical role of the inflammatory/arteriogenic pathway. Arterioscler Thromb Vasc Biol. 2006; 26:2483-9.

279. Van Keulen JK, Timmers L, Van Kuijk LP, Retnam L, Hoefer IE, Pasterkamp G, Lim SK, De Kleijn DP V. The Nuclear Factor-kappa B p50 subunit is involved in flow-induced outward arterial remodeling. Atherosclerosis. 2009; 202:424-30.

280. Castier Y, Ramkhelawon B, Riou S, Tedgui A, Lehoux S. Role of NF-kappaB in flowinduced vascular remodeling. Antioxid Redox Signal. 2009; 11:1641-9. 
281. Tzima E, Del Pozo MA, Kiosses WB, Mohamed S a, Li S, Chien S, Schwartz MA. Activation of Rac1 by shear stress in endothelial cells mediates both cytoskeletal reorganization and effects on gene expression. EMBO J. 2002; 21:6791-800.

282. Jalali S, Li Y-S, Sotoudeh M, Yuan S, Li S, Chien S, Shyy JY-J. Shear Stress Activates p60src-Ras-MAPK Signaling Pathways in Vascular Endothelial Cells. Arteriosclerosis, Thrombosis, and Vascular Biology. 1998; 18:227-234.

283. Shay-Salit A, Shushy M, Wolfovitz E, Yahav H, Breviario F, Dejana E, Resnick N. VEGF receptor 2 and the adherens junction as a mechanical transducer in vascular endothelial cells. Proc Natl Acad Sci U S A. 2002; 99:9462-7.

284. Gruionu G, Hoying JB, Pries AR, Secomb TW. Structural remodeling of mouse gracilis artery after chronic alteration in blood supply. Am J Physiol Heart Circ Physiol. 2005; 288:H2047-54.

285. Gruionu G, Hoying JB, Pries AR, Secomb TW. Structural Remodeling of the Mouse Gracilis Artery: Coordinated Changes in Diameter and Medial Area Maintain Circumferential Stress. Microcirculation. 2012; 4:no-no.

286. Adamson RH, Sarai RK, Altangerel A, Clark JF, Weinbaum S, Curry F-RE. Microvascular permeability to water is independent of shear stress, but dependent on flow direction. $A m$ J Physiol Heart Circ Physiol. 2013;:ajpheart.00956.2012-.

287. Passerini AG, Milsted A, Rittgers SE. Shear stress magnitude and directionality modulate growth factor gene expression in preconditioned vascular endothelial cells. J Vasc Surg. 2003; 37:182-90.

288. Simmers MB, Pryor AW, Blackman BR. Arterial shear stress regulates endothelial celldirected migration, polarity, and morphology in confluent monolayers. Am J Physiol Heart Circ Physiol. 2007; 293:H1937-46.

289. Wojciak-Stothard B, Ridley AJ. Shear stress-induced endothelial cell polarization is mediated by Rho and Rac but not Cdc42 or PI 3-kinases. J Cell Biol. 2003; 161:429-39.

290. Troidl K, Rüding I, Cai W-J, Mücke Y, Grossekettler L, Piotrowska I, Apfelbeck H, Schierling W, Volger OL, Horrevoets AJ, Grote K, Schmitz-Rixen T, Schaper W, Troidl C. Actin-binding rho activating protein (Abra) is essential for fluid shear stress-induced arteriogenesis. Arterioscler Thromb Vasc Biol. 2009; 29:2093-101.

291. Buch T, Heppner FL, Tertilt C, Heinen TJAJ, Kremer M, Wunderlich FT, Jung S, Waisman A. A Cre-inducible diphtheria toxin receptor mediates cell lineage ablation after toxin administration. Nat Methods. 2005; 2:419-26.

292. Regensteiner JG, Wolfel EE, Brass EP, Carry MR, Ringel SP, Hargarten ME, Stamm ER, Hiatt WR. Chronic changes in skeletal muscle histology and function in peripheral arterial disease. Circulation. 1993; 87:413-21. 
293. Brass EP, Hiatt WR. Acquired skeletal muscle metabolic myopathy in atherosclerotic peripheral arterial disease. Vasc Med. 2000; 5:55-9.

294. Burke CW, Suk JS, Kim AJ, Hsiang Y-HJ, Klibanov AL, Hanes J, Price RJ. Markedly enhanced skeletal muscle transfection achieved by the ultrasound-targeted delivery of non-viral gene nanocarriers with microbubbles. J Control Release. 2012; 162:414-21.

295. Mitchell RG, Duscha BD, Robbins JL, Redfern SI, Chung J, Bensimhon DR, Kraus WE, Hiatt WR, Regensteiner JG, Annex BH. Increased levels of apoptosis in gastrocnemius skeletal muscle in patients with peripheral arterial disease. Vasc Med. 2007; 12:285-90.

296. Lucitti JL, Mackey JK, Morrison JC, Haigh JJ, Adams RH, Faber JE. Formation of the Collateral Circulation is Regulated by Vascular Endothelial Growth Factor-A and A Disintegrin and Metalloprotease Family Members 10 and 17. Circ Res. 2012; 